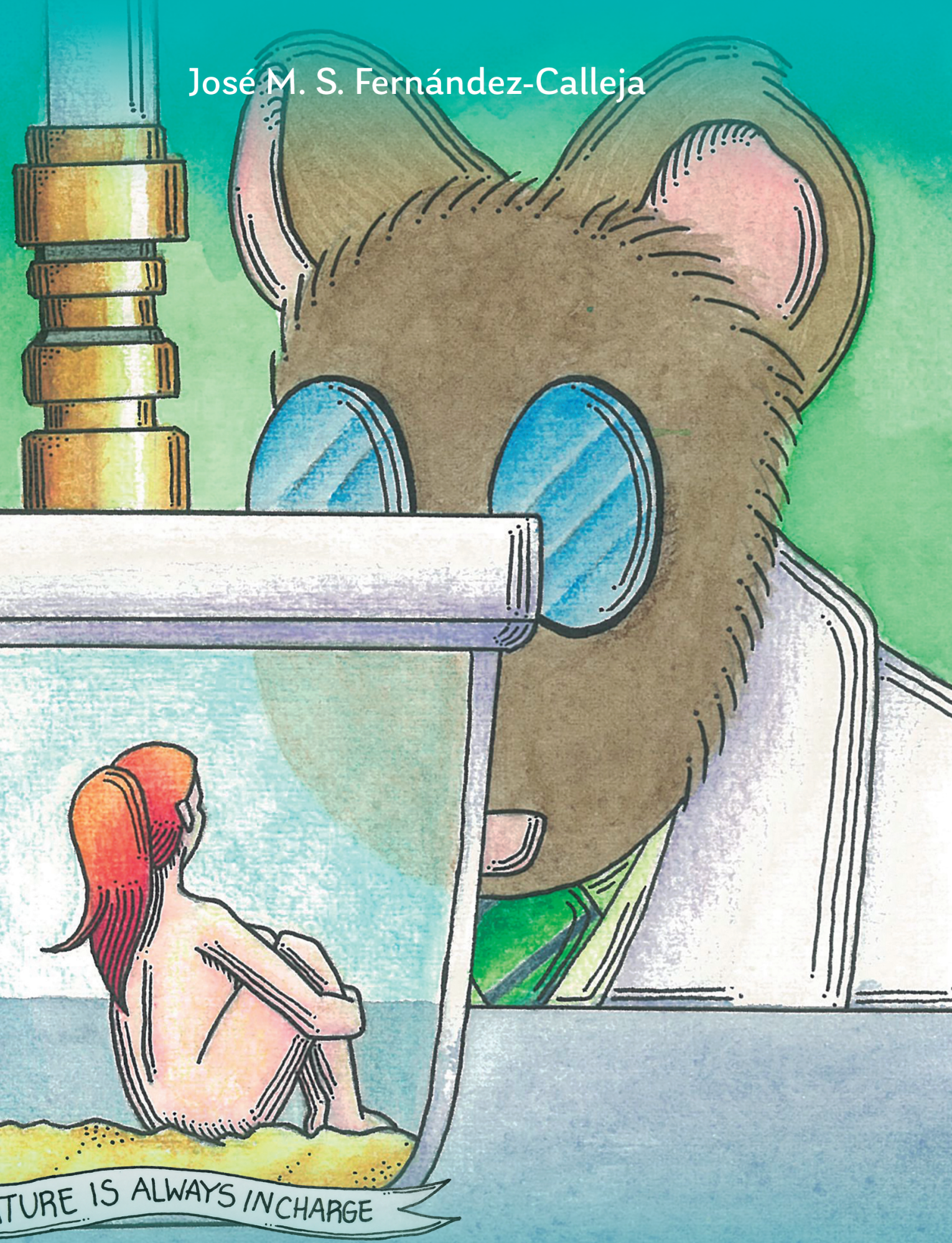

EXTENDED INDIRECT CALORIMETRY AS A PHYSIOLOGICAL PHENOTYPING TOOL IN MOUSE NUTRITIONAL INTERVENTION STUDIES, WITH A FOCUS ON METABOLIC PROGRAMMING BY STARCHES 



\section{Propositions}

1. Incorporating new gas sensors into mouse indirect calorimetry systems not only serves scientific progress but also contributes to the refinement principle in humane animal research.

(this thesis)

2. Childhood and adolescence should be recognised as sensitive periods of development within the theoretical framework of nutritional programming.

(this thesis)

3. If we want to keep using the word "race" in the human biomedical sciences we must make clear whether we refer to the biological definition or the social construct.

4. Tiny effects of the gut microbiome on obesity can still help your way into Nature if you present them as percentages of fat gain (Turnbaugh et al., Nature 2006).

5. The multiverse theory is not scientific.

6. Before we can study metabolically healthy obesity (Cardenas-Sanchez et al., J Adolesc Health 2019) we should have a solid definition of health.

7. Today's scientists should be more like the scientists of yore, marvelled at the sight of a new star in the firmament rather than a new star in our statistical outputs.

8. Female mice are the most unsuspected victims of male chauvinism in science.

Propositions belonging to the thesis, entitled

Extended indirect calorimetry as a physiological phenotyping tool in mouse nutritional intervention studies, with a focus on metabolic programming by starches

José M. S. Fernández-Calleja

Wageningen, 19 November 2019 

Extended indirect calorimetry as a physiological phenotyping tool in mouse nutritional intervention studies, with a focus on metabolic programming by starches 


\section{Thesis committee}

\section{Promotor}

Prof. Dr Jaap Keijer

Professor of Human and Animal Physiology

Wageningen University \& Research

\section{Co-promotors}

Dr Evert M. van Schothorst

Associate professor, Human and Animal Physiology

Wageningen University \& Research

Dr Annemarie Oosting

Sr. Team Leader, Developmental Biology \& Nutritional Programming, Early Life Nutrition Danone Nutricia Research, Nutricia Advanced Medical Nutrition, Utrecht

\section{Other members}

Prof. Dr Michiel Kleerebezem, Wageningen University \& Research

Prof. Dr Paula Oliver, University of the Balearic Islands, Spain

Prof. Dr Ko Willems van Dijk, Leiden University Medical Center

Dr Wilma Steegenga, Wageningen University \& Research

This research was conducted under the auspices of the Graduate School VLAG (Advanced studies in Food Technology, Agrobiotechnology, Nutrition and Health Sciences). 


\title{
Extended indirect calorimetry as a physiological phenotyping tool in mouse nutritional intervention studies, with a focus on metabolic programming by starches
}

\author{
José M. S. Fernández-Calleja
}

\section{Thesis}

submitted in fulfilment of the requirements for the degree of doctor at Wageningen University

by the authority of the Rector Magnificus,

Prof. Dr A.P.J. Mol, in the presence of the

Thesis Committee appointed by the Academic Board to be defended in public on Tuesday 19 November 2019 at 1:30 p.m. in the Aula. 
José M. S. Fernández-Calleja

Extended indirect calorimetry as a physiological phenotyping tool in mouse nutritional intervention studies, with a focus on metabolic programming by starches,

192 pages.

PhD thesis, Wageningen University, Wageningen, the Netherlands (2019)

With references, with summary in English and Spanish

ISBN 978-94-6395-111-1

DOI 10.18174/499124 


\section{Table of Contents}

Chapter $1 \quad$ General Introduction 7

Chapter $2 \quad$ Non-invasive continuous real-time in vivo analysis of microbial hydrogen production shows adaptation to fermentable carbohydrates in mice

Chapter 3 Extended indirect calorimetry with isotopic $\mathrm{CO} 2$ sensors for prolonged and continuous quantification of exogenous vs total substrate oxidation in mice

Chapter 4 A lowly digestible-starch diet after weaning enhances exogenous glucose oxidation rate in female, but not in male, mice

Chapter 5 Direct and long-term metabolic consequences of lowly vs highly digestible- starch in the early post-weaning diet of mice

Chapter 6 General Discussion

About the author

Curriculum vitae 189

List of publications 190

Education statement 



\section{Chapter}

1

\section{General Introduction}



In this Chapter, I will present the fundamental concepts and recurring themes of this thesis. First, the theoretical background of indirect calorimetry ( $\ln \mathrm{Ca}$ ) and some shortcomings of this technique are introduced. Then, the known health implications of dietary starches and their digestibility are introduced, emphasising on their apparent sex-dependent effects indicated by rodent models. We then move on to the concept of metabolic programming and how the potential of post-weaning starches to modulate metabolic health later in life is understudied. Having identified technological and scientific knowledge gaps in these areas, the aims of this thesis are delineated, along with a brief description of how these aims were addressed in each Chapter.

\section{Indirect calorimetry}

InCa is a technique used to estimate energy expenditure (EE) at the whole body level based on measurement of oxygen $\left(\mathrm{O}_{2}\right)$ consumption and carbon dioxide $\left(\mathrm{CO}_{2}\right)$ production. In the cell, acetyl-CoA derived from glucose, fatty acids, and amino acids is used to produce ATP in a series of redox reactions that consume $\mathrm{O}_{2}$ and produce $\mathrm{CO}_{2}$ and water. The commercial application of this technique to human physiology goes back to the beginning of the $20^{\text {th }}$ century ${ }^{1}$, and is at the centre of numerous metabolic studies in humans and other animals, including rodents ${ }^{2}$, production animals ${ }^{3}$, fish $^{4}$, birds ${ }^{5}$ and insects $s^{6,7}$. InCa remains the gold standard to measure energy expenditure in clinical settings $s^{8,9}$, despite the availability of other well-established techniques (e.g. direct calorimetry and the double labelled water method, DLW). While these and other alternatives are valuable in certain situations, their intrinsic challenges and limitations have favoured the use of InCa to estimate $\mathrm{EE}^{10}$. For example, measuring $\mathrm{O}_{2}$ and $\mathrm{CO}_{2}$ exchange by InCa can be done much more accurately than measuring heat production by direct calorimtery ${ }^{10}$, and EE estimated by $\mathrm{InCa}$ is not affected by macronutrient imbalance, a common source of error in DLW studies when unaccounted for $^{11}$. InCa is based on long-established notions, like the conservation of energy in closed systems, and assumptions, like the precise stoichiometry of substrate oxidation reactions. Thus, the volumes of $\mathrm{O}_{2}$ and $\mathrm{CO}_{2}$ can be used to calculate the rate at which a mixture of nutrients is utilised by the body to produce ATP.

InCa has evolved across scientific fields. The first commercial InCa systems in the 1920s were only capable of measuring $\mathrm{O}_{2}$, and only decades later were they able to accurately measure both $\mathrm{O}_{2}$ and $\mathrm{CO}_{2}$ thanks to the invention of gas analysers that did not rely on material balance ${ }^{12}$. Further developments led the design of portable and inexpensive devices for humans ${ }^{12}$, as well as airtight respiration chambers for both production animals and humans, ideal when free movement or grouped housing are needed ${ }^{13}$. In rodents, InCa systems (currently produced by three main manufacturers, Columbus Instruments International, TSE Systems, and Sable Systems International) not only offer robust measurements of gas exchange, but also simultaneous records of food intake, temperature, and physical activity ${ }^{10}$. For mice in particular, there is consistent progress in developing the InCa technique in theory and practice. For example, efforts are ongoing to increase the time resolution of $\mathrm{O}_{2}$ and $\mathrm{CO}_{2}$ measurements ${ }^{2}$, improve the interpretation of $\mathrm{EE}$ in light of body mass and compositional data ${ }^{2,14}$, and even to validate $\mathrm{EE}$ measurements without knowledge of $\mathrm{CO}_{2}$ production ${ }^{15}$. Clearly, InCa is a current, non-invasive, and evolving 
technique that offers researchers working in rodent models an excellent tool to study whole body metabolism.

For each of the different substrates, coefficients for nutrient oxidation (Table 1) represent the basis of InCa, and are used to calculate EE and substrate use under many experimental conditions. The oxidation of carbohydrate and fat mixtures is easily estimated based on the ratio of $\mathrm{CO}_{2}$ production to $\mathrm{O}_{2}$ consumption, called respiratory quotient (RQ, when referring to cellular metabolism) or respiratory exchange ratio (RER, for whole body level metabolism). Thus, an RER of 1 indicates net carbohydrate oxidation and 0.7 indicates net fat oxidation, based on the stoichiometry of oxidation reactions. For instance, $6 \mathrm{~mol}$ of $\mathrm{CO}_{2}$ are produced and $6 \mathrm{~mol}$ of $\mathrm{O}_{2}$ are consumed in the complete combustion of $1 \mathrm{~mol}$ of glucose $\left(\mathrm{CO}_{2} / \mathrm{O}_{2}=1.0\right)$, and $16 \mathrm{~mol}$ of $\mathrm{CO}_{2}$ are produced and $23 \mathrm{~mol}$ of $\mathrm{O}_{2}$ are consumed for every mol of palmitate oxidised $\left(\mathrm{CO}_{2} / \mathrm{O}_{2}=0.7\right)$. As protein oxidation is generally constant and not including this process overestimates EE very marginally ${ }^{2}$, protein oxidation is often neglected in rodent studies and referred to as non-protein RER. For the remainder of this thesis, I will use the term RER to refer to non-protein RER. Many other coefficients and formulas have been developed to account for the oxidation of unusual substrates (e.g. alcohol) and other products of substrate oxidation reactions (e.g. urea, ketone bodies) ${ }^{16}$. Some of these substrates and products may become relevant under certain circumstances. For instance, a correction for biosynthesis of ketone bodies excreted in urine and breath (thus, exceeding their oxidation) may be in order when subjects consume ketogenic diets ${ }^{17}$. Therefore, $\mathrm{O}_{2}$ and $\mathrm{CO}_{2}$ measurements safely indicate metabolic rate and the net balance of substrates oxidised at the whole body level in most conditions. But what relevant phenotypic information are we missing when we only measure $\mathrm{O}_{2}$ and $\mathrm{CO}_{2}$ ?

Table 1. Calorimetric coefficients used for the calculations of EE and fuel utilisation

$\mathrm{O}_{2}$ consumed $\mathrm{CO}_{2}$ produced Energy produced Energy equivalent of $\mathrm{O}_{2} \quad$ RQ

\begin{tabular}{cccccc} 
& $\left(1 \mathrm{~g}^{-1}\right)$ & $\left(1 \mathrm{~g}^{-1}\right)$ & $\left(\mathrm{kJ} \mathrm{g}^{-1}\right)$ & $\left(\mathrm{kJ} \mathrm{l}^{-1}\right)$ & \\
\hline Carbohydrate & 0.89 & 0.89 & 17.48 & 21.12 & 1.000 \\
\hline Fat & 2.02 & 1.43 & 39.60 & 19.61 & 0.710 \\
\hline Protein & 1.03 & 0.85 & 20.18 & 19.48 & 0.833
\end{tabular}

Based on Elia and Livesey ${ }^{16}$. These coefficients vary depending on the exact composition of model carbohydrates, fat, and proteins. A glucan residue, dioelylpalmitate, and Kleiber's standard protein, respectively, are reported in this table. Respiratory quotient (RQ), in contrast to respiratory exchange ratio (RER), is the preferred term here because RQ is independent of non-metabolic processes (hypo- or hyper-ventilation or changes in the bicarbonate pool).

This thesis is focussed on two pertinent examples where current mouse InCa systems could be improved to study the phenotype of the animal in a better integrated manner, beyond whole body metabolism. Firstly, one way in which InCa systems could be extended to obtain more complete information about substrate metabolism is to include analysis of fermentation (summarised in Figure 1). The gut microbiota obtain nutrients from the host's diet or the host itself (e.g. mucins) for microbial growth and energy production, a process generally called fermentation ${ }^{18}$. Some of the by-products generated in fermentation are waste and some can be used by both microbes and host to e.g. obtain more energy and 
modulate cellular processes. Major gaseous products of fermentation are hydrogen $\left(\mathrm{H}_{2}\right)$, methane $\left(\mathrm{CH}_{4}\right)$, and hydrogen sulfide $\left(\mathrm{H}_{2} \mathrm{~S}\right)$. The stoichiometry of fermentation is complex, but in general $\mathrm{H}_{2}$ is produced by hydrogenogens and subsequently used by methanogens (archaea) releasing $\mathrm{CH}_{4}$, acetogens to produce acetate, or sulfate reducers releasing $\mathrm{H}_{2} \mathrm{~S}$ (ref. 19). If the carbohydrates ingested by the host are fermentable, some $3-5 \%$ of the energy contained in these carbohydrates will be excreted in the faeces and fermentation gases ${ }^{20}$. Energy lost as $\mathrm{H}_{2}\left(-12.75 \mathrm{~kJ} \mathrm{l}^{-1}\right.$, or $\left.25.50 \mathrm{~kJ} \mathrm{l}^{-1} \mathrm{O}_{2}\right)$ and $\mathrm{CH}_{4}\left(-39.71 \mathrm{~kJ} \mathrm{l}^{-1}\right.$, or $19.86 \mathrm{~kJ}$ $\mathrm{I}^{-1} \mathrm{O}_{2}$ ) can be adjusted for in InCa equations for the calculation of energy expenditure and substrate oxidation ${ }^{16,21}$, although this is mostly done for ruminants as they heavily rely on fermentation. There have been sporadic studies measuring $\mathrm{H}_{2}$ and $\mathrm{CH}_{4}$ production in rats and mice, but this has been done outside the context of energy metabolism ${ }^{22-26}$ or only at discrete time points ${ }^{27,28}$. Furthermore, the importance of measuring fermentation gases goes beyond energy balance as they could provide a non-invasive way to study diet-hostmicrobiota interactions. As relevant as they might be, there are currently no commercial rodent systems capable of measuring the fermentation gases $\mathrm{H}_{2}$ and $\mathrm{CH}_{4}$ in real time or in conjunction with other physiological gases.

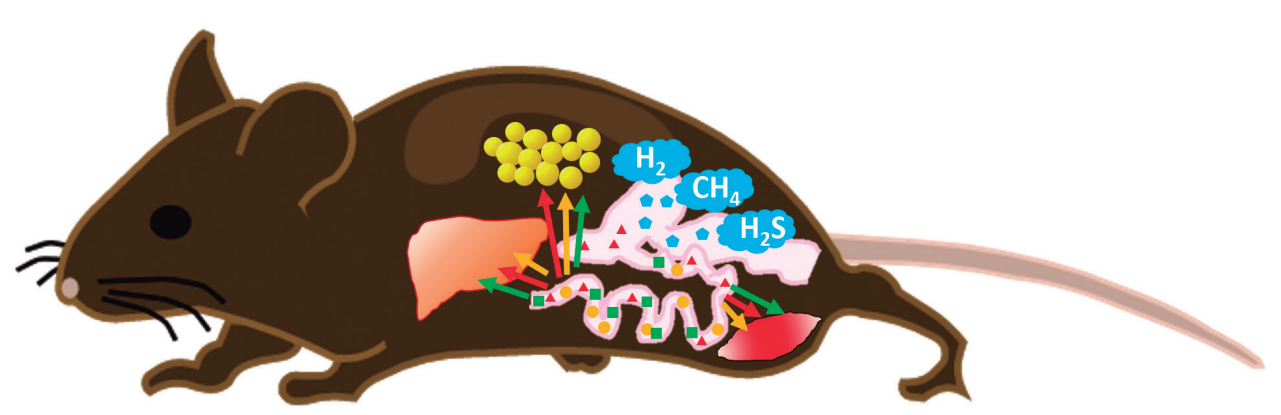

Figure 1. Where fermentation and host energy metabolism meet. Carbohydrates (red triangles), fats (orange circles), and proteins (green squares) consumed by the host go through the digestive process, starting from the mouth (not shown) and culminating in the small intestine. These nutrients are absorbed and delivered to the periphery to serve in biological processes like synthesis of structural molecules, energy storage (e.g. fat storage in white adipose tissue, WAT), and cellular and mitochondrial energy production (consuming $\mathrm{O}_{2}$ and releasing $\mathrm{CO}_{2}$ in the process). Some nutrients that resist digestion reach compartments of the gastrointestinal tract abundant in microbes, who in turn are capable to ferment these nutrients and produce metabolites like short-chain fatty acids (SCFA, blue pentagons), and gases like $\mathrm{H}_{2}, \mathrm{CH}_{4}$, and $\mathrm{H}_{2} \mathrm{~S}$. SCFA can be excreted, utilised by other microbes, or absorbed by the host (not shown) for the production of energy. SCFA can also act as molecular signals to several tissues like WAT, liver, and muscle. Fermentation gases can be excreted directly, feed microbial metabolic pathways (especially $\mathrm{H}_{2}$ ), or be absorbed in the blood stream to be ultimately exhaled via the lungs.

The second example pertains to the measurement of the oxidation of specific substrates. RER computed from $\mathrm{VO}_{2}$ (the volumetric rate of $\mathrm{O}_{2}$ consumption) and $\mathrm{VCO}_{2}$ (the volumetric rate of $\mathrm{CO}_{2}$ production) only reflects the overall oxidation of substrates in the body and thus lacks detailed information of the specific endogenous or exogenous substrates being oxidized. A way to partly overcome this limitation is to use ingested or infused isotopic tracers $^{29}$. Isotopes are atoms of a given element varying in the amount of neutrons, and they can be radioactive (e.g. ${ }^{14} \mathrm{C}$ ) or stable (non-radioactive, e.g. ${ }^{13} \mathrm{C}$ ). Isotopic tracers are 
compounds (e.g. ${ }^{13} \mathrm{C}$-labelled glucose) that are metabolised in the same way as their tracee (e.g. normal glucose), but that can be differentially detected (e.g. based on their radioactivity or mass difference). Isotopic enrichment can be achieved artificially (e.g. by growing plants in ${ }^{13} \mathrm{CO}_{2}$ rich atmospheres), but it is also present in nature at lower levels. For instance, $\mathrm{C} 4$ plants like maize are naturally enriched in ${ }^{13} \mathrm{C}$ compared to $\mathrm{C} 3$ plants like wheat ${ }^{30}$. Thus, it is possible to distinguish between oxidation of endogenous (body) and exogenous (ingested) fuels using isotopic tracers in combination with a method to detect the resulting isotopically labelled $\mathrm{CO}_{2}$. This distinction is important when one wishes to measure the oxidative disposal of newly ingested nutrients irrespective of their availability in body stores, e.g. in studying how recently ingested nutrients are used to fuel physical activity ${ }^{31,32}$, or when the (altered) oxidation of specific nutrients may indicate a (patho) physiological condition, like Helicobacter pylori infection or delayed gastric emptying assessed by ${ }^{13} \mathrm{CO}_{2}$ breath tests using ${ }^{13} \mathrm{C}$ urea and ${ }^{13} \mathrm{C}$ octanoic acid, respectively ${ }^{29}$.

Nowadays stable isotopes are preferred over radioactive isotopic tracers due to health concerns ${ }^{33}$. This also means that the method of detection of isotopically-labelled $\mathrm{CO}_{2}$ became more difficult: ${ }^{14} \mathrm{CO}_{2}$ can be readily measured with a scintillation counter, but ${ }^{13} \mathrm{CO}_{2}$ is usually detected by expensive mass spectroscopy ${ }^{30}$. To complicate matters, breath samples can be stored for future analysis, but the decision of how many samples are to be collected at what intervals must be based on a priori knowledge of the expected outcomes (e.g. oxidation of glucose goes much faster than oxidation of fat) and, of course, the ability to collect those samples. A good example here is an ambitious paper on mouse metabolism using stable isotopic tracers, where 13 people were acknowledged only for collecting hourly breath samples for ${ }^{13} \mathrm{CO}_{2}$ enrichment analysis ${ }^{34}$. Although studies using metabolic tracers and measuring ${ }^{13} \mathrm{CO}_{2}$ enrichment are present, only two have combined this with $\ln \mathrm{Ca}^{34,35}$, which is a prerequisite to quantify true oxidation rates because ${ }^{13} \mathrm{CO}_{2}$ enrichment only provides qualitative data ${ }^{36}$. The problem is not, however, that there are no other ways of measuring ${ }^{13} \mathrm{CO}_{2}$. There are in fact other real-time, less expensive ways ${ }^{30}$, and at least one of them has been implemented already in InCa studies on production animals ${ }^{37}$. But, for some unknown reason and despite the wide availability of commercial InCa systems for rodents, there are currently no systems on the market that integrate ${ }^{13} \mathrm{CO}_{2}$ enrichment analysis into InCa.

\section{Starches}

Starches are polymers of glucose produced by plants, and as such, they constitute a major component of human diets. There are two main starch polymers and both are present in almost all starch sources: amylose and amylopectin (Figure 2). Amylose is a non-branching chain linked by $\alpha-1,4^{\prime}$ glycosidic bonds, and amylopectin is very branched and linked by both $\alpha-1,4^{\prime}$ and $\alpha-1,6^{\prime}$ glycosidic bonds ${ }^{38}$. The content of amylose and amylopectin in starch sources and the configuration they take as crystalline granules is variable and influences the ability of salivary and pancreatic amylase to digest these starches ${ }^{38}$. Starch digestibility can also be influenced by processing, for instance, heating starches in water disrupts their crystalline structure and renders them more digestible ${ }^{39}$. 
AMYLOSE

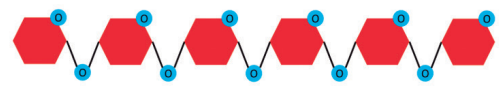

\section{HYDROLYISIS PRODUCTS}

$\alpha-$ Maltotriose

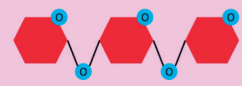

$\alpha$-Maltose

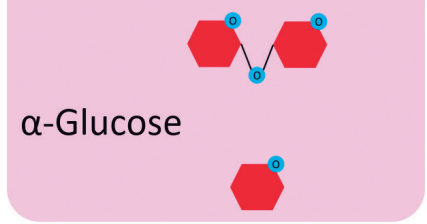

AMYLOPECTIN

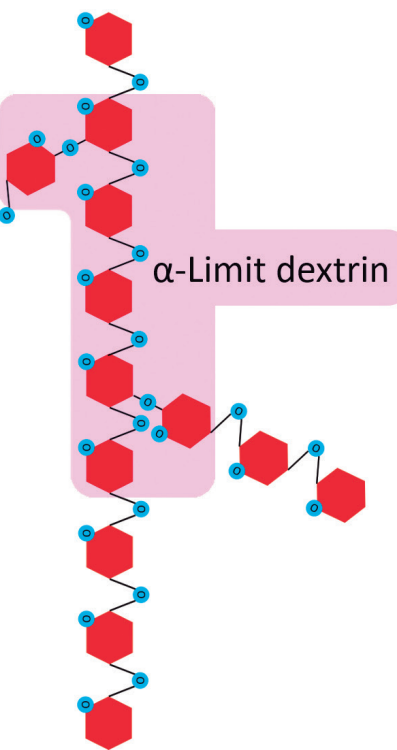

Figure 2. Simplified representation of the molecular structure of starch and starch digestion products. Amylose is a non-branching glucose polymer linked by $\alpha-1,4$ glycosidic linkages, whereas amylopectin is a branched glucose polymer linked by $\alpha-1,4$ ' and $\alpha-1,6$ ' linkages. Some of the products formed upon enzymatic digestion of starches are shown in purple. Oxygen atoms in ether and glycosidic bonds are shown in blue. Based on Colonna et al. ${ }^{39}$

Based on their in vitro digestibility (using an enzyme mixture containing $\alpha$-amylase) and observations in human ileostomies, starch fractions have been classified as rapidly digestible starch (RDS, digested within 20 min), slowly digestible starch (SDS, 20 - 120 min), and resistant starch (RS, not digested within $120 \mathrm{~min}$ and assumed to escape absorption by the small intestine $)^{40}$. A single starch source may form different proportions of RDS, SDS, and RS, and starch sources high in amylose are more likely to form RS ${ }^{18}$. This classification is however not universally accepted nor consistently used throughout the scientific literature ${ }^{41,42}$. Therefore, I will circumvent using these definitions by referring to starches simply as lowly digestible or highly digestible starches.

The rate of starch digestion can affect postprandial glycaemia and gut-microbial activity. The glycaemic response to foods is scored by the glycaemic index ( $\mathrm{Gl}$ ), a measure of how blood glucose levels change after a meal ${ }^{43}$. Lowly digestible starches can sometimes ${ }^{44}$, but not always ${ }^{45}$, be low $\mathrm{Gl}$ foods. Undigested starch can thus potentially become available to gut microbes as a substrate for fermentation, resulting in the production of gases (e.g. $\mathrm{H}_{2}, \mathrm{CH}_{4}$ ) and other metabolites ${ }^{46}$. A class of these metabolites are the group of organic acids known as short-chain fatty acids (SCFAs). The main SCFAs are acetate, propionate, and butyrate, and each of them can have different physiological effects. For example, the liver uses acetate to obtain energy and synthesise lipids, and propionate to synthesise glucose $^{47}$, and colonocytes obtain energy from butyrate oxidation ${ }^{48}$.

Human and rodent studies indicate that starch digestibility may have important health consequences. For instance, lowly digestible starches could be helpful in managing diabetes and reduce obesity and colon cancer risk ${ }^{49}$. It is thought that these effects could 
be mediated by a reduced glycaemic response to lowly digestible starches, dilution of the energy density of foods, increased satiety, increased fat oxidation, and their impact on gut microbiota composition and activity (e.g. production of SCFAs $)^{42,49}$. While the evidence for these effects in humans is very limited and still needs to be complemented by more long-term feeding studies ${ }^{50}$, evidence that lowly digestible starches have a positive impact on metabolic health in rodent models is promising. One of the most consistent effects of lowly us highly digestible starches in male rodents is a decrease in adiposity ${ }^{51}$. White adipose tissue (WAT) is an important organ in the regulation of energy homeostasis, through its capacity to store energy in the form of triglycerides and its endocrine functions (i.e. secretion of adipokines like leptin and adiponectin). Feeding lowly us highly digestible starches has been shown to preserve the function of WAT by preventing adipocyte hypertrophy ${ }^{52,53}$, maintaining adipocyte insulin sensitivity ${ }^{52,53}$, and reducing macrophage recruitment to this tissue ${ }^{54}$. Insulin resistance in WAT may also impair the capacity of an organism to adjust fuel utilisation to fuel availability, a concept known as metabolic flexibility ${ }^{55}$, by a failure of WAT to suppress lipolysis upon insulin stimulation. At the same time, increased macrophage infiltration in WAT can indicate an inflammatory status and has been associated with a reduced metabolic flexibility ${ }^{56}$. There is indeed evidence that lowly digestible starches preserve metabolic flexibility, as seen by a greater switch to glucose oxidation in response to a carbohydrate-rich meal in male mice that had been on a lowly us highly digestible starch diet for several weeks ${ }^{57}$.

Although the physiological effects of starches have been a subject of study for decades, it is poorly recognised that these effects may be different in females and males. Studies like the one by Robertson et al..$^{58}$ show that the same starchy meal is oxidised more extensively by women than by men, and that this is probably due to the higher insulin sensitivity of women. How starch digestibility and sex interact is even less studied, and the available evidence comes from rodent models of dietary GI interventions. From these studies (reviewed by Campbell et al..$^{51}$ ) it can be concluded that body weight, body fat, and glucose tolerance are improved by low us high GI diets (mainly represented by lowly us highly digestible starches) only in males, although less than a quarter of the available studies included females. Moreover, other aspects of glucose metabolism besides glucose tolerance and fasting glucose and insulin levels have not been sufficiently studied in both sexes. For example, male mice have an better capacity to oxidise exogenous glucose after long-term consumption of lowly digestible starches compared to males fed a highly digestible starch $\operatorname{diet}^{57}$. This is, however, not known for females. Therefore, an important step to understand the metabolic implications of dietary starches would be to provide more evidence for headto-head comparisons of the physiological effects of starches of different digestibility in females us males.

\section{Metabolic programming}

It is increasingly recognised that not everyone living in an unhealthy environment is equally vulnerable to it. It is thought that disease risk late in life is in part due to different environmental influences (including nutrition) early in development, according to the developmental origins of health and disease $(\mathrm{DOHaD})$ theory ${ }^{59,60}$. This phenomenon 
(sometimes also referred to as metabolic, nutritional, or developmental programming) has been repeatedly demonstrated, from its first associations based on historical demographical data from 1934 (ref. 61), to the experimental work linking early life environment to cardiovascular and metabolic disease in large and small animals in the 1970s ${ }^{59}$, to epidemiological work on foetal growth restriction and postnatal cardiovascular disease risk $^{62}$, to recent advances on the epigenetic mechanisms driving metabolic programming ${ }^{63}$.

For these exposures or environmental cues to have a long-lasting impact, it is key that they take place during periods of developmental plasticity, like perinatal life and early childhood. This is a good reason why policy makers are now taking a life-course approach to public health, targeting sensitive periods of development like childhood ${ }^{64}$, and why scientific journals are devoting special attention to the role of health during critical stages of life in the prevention of non-communicable diseases ${ }^{65,66}$. Experimental animal studies are critical to progress research in the $\mathrm{DOHaD}$ field as it requires early interventions, but its effects on later life require model animals with a short life cycle. For instance, adjusting the precise types and quantities of nutrients in infant formulas can only be reconsidered if there is sufficient backing from model organisms about safety and efficacy. In this regard, mice and rats are very useful models to investigate nutritional programming effects, particularly because of their relatively quick generation time and the vast amount of metabolic and molecular knowledge and tools available for these species ${ }^{10,67}$. We indeed owe a large deal of our understanding of metabolic programming mechanisms to rodent studies $^{68,69}$. Promisingly, at least one dietary concept to prevent adult obesity starting in early life, i.e. the modification of the physical structure of milk fat globules in infant formula to resemble breast milk more closely, has been successfully taken from mouse models to the first clinical trials in humans ${ }^{70,71}$.

Despite the fact that the sensitivity of the developing organism to nutritional stimuli is undisputed, there is still little scientific evidence to recommend macronutrient intakes for infants and young children. The case of carbohydrates is particularly understudied. The proportion of dietary carbohydrates dramatically increases in the transition from lactation to complementary feeding ${ }^{72}$, yet all we know is that added sugars should be avoided to prevent caries and that because high glycaemic responses can be detrimental for adults we should assume high-glycaemic index foods are also negative for the young ${ }^{73}$. In this regard, human milk, containing lactose, produces a low glycaemic response ${ }^{74}$. Moreover, the introduction of solid foods during weaning is the greatest determinant of early life gut microbiota composition ${ }^{75}$. The development of a healthy symbiosis between the gut microbiota and the host in this period could determine the risk of obesity and other noncommunicable diseases $^{76}$. Accordingly, an altered gut microbiota composition by exposure to antibiotics during the gestation, lactation, and early post-weaning periods has been shown to promote obesity in mice ${ }^{77}$. In this sense, the type of starches that babies are weaned onto could have metabolic programming effects on later life health. At present, there is only one starch-based study that has directly asked this kind of question using a rat model, and the results suggest that lowly digestible starches given to pregnant dams and to their offspring during early life may protect females from metabolic impairment, as shown by the better glucose tolerance and lower visceral adiposity of female offspring at 
the end of the study ${ }^{78}$. This study, however, was mainly focussed on the role of maternal diet and the study was terminated at the beginning of adulthood. Whether long-term effects on metabolic health can be induced solely by the digestibility of the starches first encountered by an organism and whether those effects persist well beyond adolescence remains to be tested.

\section{Aims}

The overall aims of this thesis are twofold: 1) to develop and show the added value of an extended mouse metabolic phenotyping tool based on InCa for the real-time study of microbiota activity and the oxidation of exogenous us endogenous substrates; and 2) to apply this tool to study the direct and metabolic programming effects of starches consumed during the early post-weaning period.

In particular, we aim to establish whether additional gas sensors can be integrated into an InCa system, thus an extended InCa (elnCa) system, to study microbiota activity (Chapter 2) and the specific oxidation of exogenous substrates (Chapter $\mathbf{3}$ ). We also aim to examine whether consuming starches of low us high digestibility acutely affect the oxidation of the starch molecule, and whether this is sex-dependent (Chapter 4). Finally, we aim to explore whether and to what extent starches of different digestibility consumed during the early post-weaning period can improve metabolic health later in life, and whether this is sexdependent (Chapter 5).

Our experimental approach towards these aims is summarised as follows:

- Chapter 2 describes a proof-of-principle study of the added value of $\mathrm{H}_{2}$ and $\mathrm{CH}_{4}$ sensors coupled to a commercial InCa system. The system is tested by examining mice acutely and chronically exposed to lowly and highly digestible starch diets. Other fermentation parameters and the relationship of $\mathrm{H}_{2}$ with faecal microbial communities are explored to support the validity of the approach to study microbiota activity.

- Chapter 3 describes the performance of ${ }^{13} \mathrm{CO}_{2}$ and ${ }^{12} \mathrm{CO}_{2}$ sensors incorporated into the same commercial InCa system. The validity of the new sensors to measure ${ }^{13} \mathrm{CO}_{2}$ enrichment is tested by examining mice fed diets with natural variation in ${ }^{13} \mathrm{C}$ content. The system is further tested using lean and obese mice fed a liquid mixed meal containing ${ }^{13} \mathrm{C}$ glucose or ${ }^{13} \mathrm{C}$ palmitate to quantify exogenous substrate oxidation rates. The results are compared to conventional measurements of RER as the standard indicator of metabolic flexibility to illustrate the added value of isotopic $\mathrm{CO}_{2}$ sensors in tandem with conventional InCa in mice.

- Chapter 4 tests the hypothesis that a short-term intervention with lowly us highly digestible starches acutely influences the oxidative disposal of the starch molecule, in young female and male mice. Selected parameters related to the digestion and partitioning of a single starch bolus are further investigated in females. 
- Chapter 5 tests the hypothesis that lowly us highly digestible starches in the early post-weaning diet program the adult metabolic phenotype, in female and male mice. Metabolic phenotyping in this study includes body weight and composition, glucose tolerance, metabolic flexibility, whole body metabolism, and adipose tissue morphology and inflammation markers. Additionally, the direct effects of starch digestibility in the early post-weaning period are examined.

Finally, Chapter 6 offers a general discussion of the technological and scientific contributions of this thesis, and future perspectives on elnCa and nutritional programming studies in mouse models. 


\section{References}

Atwater, W. O. \& Benedict, F. G. A Respiration Calorimeter with Appliances for the Direct Determination of Oxygen. (Carnegie Institution of Washington, Washington, D.C., 1905).

Even, P. C. \& Nadkarni, N. A. Indirect calorimetry in laboratory mice and rats: principles, practical considerations, interpretation and perspectives. Am. J. Physiol. Regul. Integr. Comp. Physiol. $\mathbf{3 0 3 ,}$ R459-476 (2012).

Gerrits, W. J. J., Borne, J. J. G. C. v. d. \& Labussière, E. in Indirect Calorimetry Ch. 1, 19-34 (Wageningen Academic Publishers, Wageningen, 2015).

Nelson, J. A. Oxygen consumption rate v. rate of energy utilization of fishes: a comparison and brief history of the two measurements. J. Fish. Biol. 88, 10-25 (2016).

Walsberg, G. E. \& Wolf, B. O. Variation in the respiratory quotient of birds and implications for indirect calorimetry using measurements of carbon-dioxide production. J. Exp. Biol. 198, 213-219 (1995).

Yatsenko, A. S., Marrone, A. K., Kucherenko, M. M. \& Shcherbata, H. R. Measurement of metabolic rate in Drosophila using respirometry. J. Vis. Exp., e51681 (2014).

Lighton, J. R., Brownell, P. H., Joos, B. \& Turner, R. J. Low metabolic rate in scorpions: implications for population biomass and cannibalism. J. Exp. Biol. 204, 607-613 (2001).

Haugen, H. A., Chan, L. N. \& Li, F. Indirect calorimetry: a practical guide for clinicians. Nutr. Clin. Pract. 22, 377-388 (2007).

da Rocha, E. E., Alves, V. G. \& da Fonseca, R. B. Indirect calorimetry: methodology, instruments and clinical application. Curr. Opin. Clin. Nutr. Metab. Care 9, 247-256 (2006).

Speakman, J. R. Measuring energy metabolism in the mouse - theoretical, practical, and analytical considerations. Front. Physiol. 4, 34 (2013).

Hall, K. D. et al. Methodologic considerations for measuring energy expenditure differences between diets varying in carbohydrate using the doubly labeled water method. Am. J. Clin. Nutr. 109, 13281334 (2019).

Frankenfield, D. C. On heat, respiration, and calorimetry. Nutrition 26, 939-950 (2010).

Heetkamp, M. J. W. et al. in Indirect calorimetry Ch. 2, 35-56 (Wageningen Academic Publishers, Wageningen, 2015).

Tschop, M. H. et al. A guide to analysis of mouse energy metabolism. Nat. Methods 9, 57-63 (2012).

Kaiyala, K. J., Wisse, B. E. \& Lighton, J. R. B. Validation of an equation for energy expenditure that does not require the respiratory quotient. PLoS One 14, e0211585 (2019).

Elia, M. \& Livesey, G. Energy expenditure and fuel selection in biological systems: the theory and practice of calculations based on indirect calorimetry and tracer methods. World Reu. Nutr. Diet. 70 , 68-131 (1992).

Schutz, Y. \& Ravussin, E. Respiratory quotients lower than 0.70 in ketogenic diets. Am. J. Clin. Nutr. 33, 1317-1319 (1980).

Englyst, K. N., Liu, S. \& Englyst, H. N. Nutritional characterization and measurement of dietary carbohydrates. Eur. J. Clin. Nutr. 61 Suppl 1, S19-39 (2007).

Wolf, P. G., Biswas, A., Morales, S. E., Greening, C. \& Gaskins, H. R. $\mathrm{H}_{2}$ metabolism is widespread and diverse among human colonic microbes. Gut Microbes 7, 235-245 (2016).

Elia, M. \& Cummings, J. H. Physiological aspects of energy metabolism and gastrointestinal effects of carbohydrates. Eur. J. Clin. Nutr. 61 Suppl 1, S40-74 (2007). $\mathrm{D}_{2}{ }^{18} \mathrm{O}$ methodologies. Eur. J. Clin. Nutr. 50, 524-534 (1996). 
Hartmann, L., Taras, D., Kamlage, B. \& Blaut, M. A new technique to determine hydrogen excreted by gnotobiotic rats. Lab. Anim. 34, 162-170 (2000).

Ostrander, C. R., Stevenson, D. K., Neu, J., Kerner, J. A. \& Moses, S. W. A sensitive analytical apparatus for measuring hydrogen production rates. I. Application to studies in small animals. Evidence of the effects of an $\alpha$-glucosidehydrolase inhibitor in the rat. Anal. Biochem. 119, 378-386 (1982).

Dufourlescoat, C., Lecoz, Y., Andrieux, C. \& Szylit, O. Effects of nature, size and level of incorporation of dietary fibers on colonic functions in germ-free rats and in heteroxenic rats inoculated with a human flora. Food. Hydrocoll. 9, 9-15 (1995).

Rodkey, F. L., Collison, H. A. \& O’Neal, J. D. Carbon monoxide and methane production in rats, guinea pigs, and germ-free rats. J. Appl. Physiol. 33, 256-260 (1972).

Tuboly, E. et al. Determination of endogenous methane formation by photoacoustic spectroscopy. J. Breath Res. 7, 046004 (2013).

Isken, F., Klaus, S., Osterhoff, M., Pfeiffer, A. F. \& Weickert, M. O. Effects of long-term soluble vs. insoluble dietary fiber intake on high-fat diet-induced obesity in C57BL/6] mice. J. Nutr. Biochem. 21, 278-284 (2010).

Walter, D. J., Eastwood, M. A., Brydon, W. G. \& Elton, R. A. An experimental design to study colonic fibre fermentation in the rat: the duration of feeding. Br. J. Nutr. 55, 465-479 (1986).

Van den Borne, J., Heetkamp, M. J. W., Alferink, s. J. J. \& Gerrits, W. in Indirect Calorimetry Ch. 5, 87. 114 (Wageningen Academic Publishers, Wageningen, 2015).

McCue, M. D. \& Welch, K. C. ${ }^{13}$ C-Breath testing in animals: theory, applications, and future directions. J. Comp. Physiol. B 186, 265-285 (2015).

Jeukendrup, A. E., Mensink, M., Saris, W. H. \& Wagenmakers, A. J. Exogenous glucose oxidation during exercise in endurance-trained and untrained subjects. J. Appl. Physiol. (1985) 82, 835-840 (1997).

Sonko, B. J. et al. Ingested fat oxidation contributes $8 \%$ of 24 -h total energy expenditure in moderately obese subjects. J. Nutr. 135, 2159-2165 (2005).

McCue, M. D. Tracking the oxidative and nonoxidative fates of isotopically labeled nutrients in animals. Bioscience 61, 217-230 (2011).

McCue, M. D. \& Pollock, E. D. Measurements of substrate oxidation using ${ }^{13} \mathrm{CO}_{2}$-breath testing reveals shifts in fuel mix during starvation. J. Comp. Physiol. B. 183, 1039-1052 (2013).

Ishihara, K. et al. A soybean peptide isolate diet promotes postprandial carbohydrate oxidation and energy expenditure in type II diabetic mice. J. Nutr. 133, 752-757 (2003).

Welch, K. C., Jr., Peronnet, F., Hatch, K. A., Voigt, C. C. \& McCue, M. D. Carbon stable-isotope tracking in breath for comparative studies of fuel use. Ann. N. Y. Acad. Sci. 1365, 15-32 (2016).

Alferink, S. J. J. et al. in Progress in Research on Energy and Protein Metabolism. (Wageningen Academic Publishers, Wageningen, 2003).

Colonna, P., Leloup, V. \& Buleon, A. Limiting factors of starch hydrolysis. Eur. J. Clin. Nutr. 46 Suppl 2, S17-32 (1992). important starch fractions. Eur. J. Clin. Nutr. 46 Suppl 2, S33-50 (1992). using an in vitro model integrating intestinal mucosal enzymes. Food Nutr. Sci. 09, 711-734 (2018). 


\section{CHAPTER 1}

44 Zhang, G. \& Hamaker, B. R. Slowly digestible starch: concept, mechanism, and proposed extended glycemic index. Crit. Reu. Food Sci. Nutr. 49, 852-867 (2009).

Eelderink, C. et al. Slowly and rapidly digestible starchy foods can elicit a similar glycemic response because of differential tissue glucose uptake in healthy men. Am. J. Clin. Nutr. 96, 1017-1024 (2012).

Englyst, K. N. \& Englyst, H. N. Carbohydrate bioavailability. Brit. J. Nutr. 94, 1 (2007).

den Besten, G. et al. The role of short-chain fatty acids in the interplay between diet, gut microbiota, and host energy metabolism. J. Lipid. Res. 54, 2325-2340 (2013).

Bird, A. R., Brown, I. L. \& Topping, D. L. Starches, resistant starches, the gut microflora and human health. Curr. Issues Intest. Microbiol. 1, 25-37 (2000).

Birt, D. F. et al. Resistant starch: promise for improving human health. Adv. Nutr. 4, 587-601 (2013).

Robertson, M. D. Dietary-resistant starch and glucose metabolism. Curr. Opin. Clin. Nutr. Metab. Care 15, 362-367 (2012).

Campbell, G. J., Senior, A. M. \& Bell-Anderson, K. S. Metabolic effects of high glycaemic index diets: a systematic review and meta-analysis of feeding studies in mice and rats. Nutrients $\mathbf{9}, 646$ (2017).

So, P. W. et al. Impact of resistant starch on body fat patterning and central appetite regulation. PLoS One 2, e1309 (2007).

Kabir, M. et al. Dietary amylose-amylopectin starch content affects glucose and lipid metabolism in adipocytes of normal and diabetic rats. J. Nutr. 128, 35-43 (1998).

Bindels, L. B. et al. Resistant starch can improve insulin sensitivity independently of the gut microbiota. Microbiome 5, 12 (2017).

Galgani, J. E., Moro, C. \& Ravussin, E. Metabolic flexibility and insulin resistance. Am. J. Physiol. Endocrinol. Metab. 295, E1009-1017 (2008).

Sparks, L. M. et al. Relation of adipose tissue to metabolic flexibility. Diabetes Res. Clin. Pract. 83, 3243 (2009).

Isken, F. et al. Impairment of fat oxidation under high- vs. low-glycemic index diet occurs before the development of an obese phenotype. Am. J. Physiol. Endocrinol. Metab. 298, E287-295 (2010).

Robertson, M. D., Livesey, G. \& Mathers, J. C. Quantitative kinetics of glucose appearance and disposal following a ${ }^{13} \mathrm{C}$-labelled starch-rich meal: comparison of male and female subjects. Brit. J. Nutr. 87, 569 (2007).

Gluckman, P. D., Hanson, M. A. \& Buklijas, T. A conceptual framework for the developmental origins of health and disease. J. Deu. Orig. Health Dis. 1, 6-18 (2010).

Gluckman, P. D., Hanson, M. A. \& Beedle, A. S. Early life events and their consequences for later disease: a life history and evolutionary perspective. Am. J. Hum. Biol. 19, 1-19 (2007).

Kermack, W. O., McKendrick, A. G. \& McKinlay, P. L. Death-rates in Great Britain and Sweden. Some general regularities and their significance. Int. J. Epidemiol. 30, 678-683 (2001).

Barker, D. J. The fetal and infant origins of adult disease. BMJ 301, 1111 (1990). 157, 95-109 (2014).

Jacob, C. M., Cooper, C., Baird, J. \& Hanson, M. What quantitative and qualitative methods have been developed to measure the implementation of a life-course approach in public health policies at the national level?, https://www.ncbi.nlm.nih.gov/pubmed/30869851 (2019).

65 The Lancet. Campaigning for preconception health. Lancet 391 (2018). risk. Philos. Trans. R. Soc. Lond. B. Biol. Sci. 374, 20180109 (2019). 
McMullen, S. \& Mostyn, A. Animal models for the study of the developmental origins of health and disease. Proc. Nutr. Soc. 68, 306-320 (2009).

68 Seki, Y., Williams, L., Vuguin, P. M. \& Charron, M. J. Minireview: epigenetic programming of diabetes and obesity: animal models. Endocrinology 153, 1031-1038 (2012).

69 Langley-Evans, S. C. \& McMullen, S. Developmental origins of adult disease. Med. Princ. Pract. 19, 87$98(2010)$.

$70 \quad$ Breij, L. M. et al. An infant formula with large, milk phospholipid-coated lipid droplets containing a mixture of dairy and vegetable lipids supports adequate growth and is well tolerated in healthy, term infants. Am. J. Clin. Nutr. 109, 586-596 (2019).

Oosting, A. et al. Size and phospholipid coating of lipid droplets in the diet of young mice modify body fat accumulation in adulthood. Pediatr. Res. 72, 362-369 (2012).

Shaoul, R., Tiosano, D. \& Hochberg, Z. Evo-devo of child growth: the role of weaning in the transition from infancy to childhood. Crit. Reu. Food Sci. Nutr. 56, 887-895 (2016).

Stephen, A. et al. The role and requirements of digestible dietary carbohydrates in infants and toddlers. Eur. J. Clin. Nutr. 66, 765-779 (2012).

Wright, C. J., Atkinson, F. S., Ramalingam, N., Buyken, A. E. \& Brand-Miller, J. C. Effects of human milk and formula on postprandial glycaemia and insulinaemia. Eur. J. Clin. Nutr. 69, 939-943 (2015).

Tilg, H. \& Moschen, A. R. Food, immunity, and the microbiome. Gastroenterology 148, 1107-1119 (2015).

Wopereis, H., Oozeer, R., Knipping, K., Belzer, C. \& Knol, J. The first thousand days - intestinal microbiology of early life: establishing a symbiosis. Pediatr. Allergy Immunol. 25, 428-438 (2014).

Cox, L. M. et al. Altering the intestinal microbiota during a critical developmental window has lasting metabolic consequences. Cell 158, 705-721 (2014).

Gugusheff, ]. et al. The effect of maternal and post-weaning low and high glycaemic index diets on glucose tolerance, fat deposition and hepatic function in rat offspring. J. Dev. Orig. Health Dis., 1-10 (2015). 



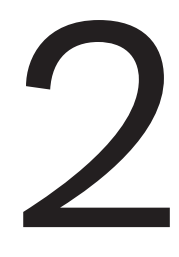

Non-Invasive Continuous Real-Time In Vivo Analysis of Microbial Hydrogen Production Shows Adaptation to Fermentable Carbohydrates in Mice

José M. S. Fernández-Calleja

Prokopis Konstanti

Hans J. M. Swarts

Lianne M. S. Bouwman

Vicenta Garcia-Campayo

Nils Billecke

Annemarie Oosting

Hauke Smidt

Jaap Keijer

Evert M. van Schothorst 



\section{Abstract}

Real time in vivo methods are needed to better understand the interplay between diet and the gastrointestinal microbiota. Therefore, a rodent indirect calorimetry system was equipped with hydrogen $\left(\mathrm{H}_{2}\right)$ and methane $\left(\mathrm{CH}_{4}\right)$ sensors. $\mathrm{H}_{2}$ production was readily detected in $\mathrm{C} 57 \mathrm{BL} / 6$ ] mice and followed a circadian rhythm. $\mathrm{H}_{2}$ production was increased within 12 hours after first exposure to a lowly-digestible starch diet (LDD) compared to a highly-digestible starch diet (HDD). Marked differences were observed in the faecal microbiota of animals fed the LDD and HDD diets. $\mathrm{H}_{2}$ was identified as a key variable explaining the variation in microbial communities, with specific taxa (including Bacteroides and Parasutterella) correlating with $\mathrm{H}_{2}$ production upon LDD-feeding. $\mathrm{CH}_{4}$ production was undetectable which was in line with absence of $\mathrm{CH}_{4}$ producers in the gut. We conclude that real-time in vivo monitoring of gases provides a non-invasive time-resolved system to explore the interplay between nutrition and gut microbes in a mouse model, and demonstrates potential for translation to other animal models and human studies.

Keywords: Indirect calorimetry, microbiota, Bacteroides, hydrogen, starch, amylose, amylopectin, dietary carbohydrates, C57BL mice, nutritional physiology. 


\section{Introduction}

Carbohydrates are a major dietary constituent of humans and rodents. Not all carbohydrates are metabolically equal. Most dietary carbohydrates, including several sugars and starches high in amylopectin content, are readily digested and thus absorbed early in the gastro-intestinal tract, making them quickly available to the organism ${ }^{1}$. Other carbohydrates, such as amylose-rich starches, are only available to the organism after fermentation by the intestinal microbiota ${ }^{2}$, which results in a more gradual release to the organism. Microbial fermentation results in a variety of metabolic products, including short chain fatty acids (SCFA), which are thought to mediate the beneficial health effects of the intestinal microbial community ${ }^{3}$. Glucose and other monosaccharides, present as such in the diet or becoming available from highly-digestible carbohydrates, are readily taken up via transporters from the small intestinal lumen into the body. This occurs primarily in the jejunum, the proximal part of the small intestine ${ }^{4}$. Carbohydrates that are less readily digestible reach the caecum and colon, where most of the intestinal microbiota reside ${ }^{5}$. Specific microbial communities utilize these substrates, in the process generating metabolites that are absorbed by the body, or are excreted as gases or in the faeces. Major digestion products are SCFA, which are known to influence host physiology, acting as energy substrates and as signalling molecules ${ }^{3}$. Other digestion products are the microbial fermentation gases hydrogen $\left(\mathrm{H}_{2}\right)$, methane $\left(\mathrm{CH}_{4}\right)$, and hydrogen sulphide $\left(\mathrm{H}_{2} \mathrm{~S}\right)^{6}$.

Since the studies of Gordon et al. ${ }^{7}$, it is increasingly realized that the small and large intestinal microbiota not only plays a major role in gastrointestinal health but also in the host's metabolic health ${ }^{8,9}$. However, how the microbial community affects metabolic health and how this can be beneficially modulated by nutrition and specific nutrients is far less well established. While a variety of cross-sectional methods can be applied to analyse changes in intestinal microbiota in rodents at specific time points, longitudinal measurements in rodent and human studies mainly rely on sampling of the faeces, long after food-microbiota interactions have already taken place throughout the gastrointestinal tract. Continuous measurements of fermentation gas emissions are already in place for ruminants like cattle and sheep ${ }^{10-12}$, as they are known to fully rely on microbiota fermentation in rumen and hindgut to digest cellulose, being distinct from monogastric organisms including rodents and humans. Furthermore, recent studies showed strong correlations between dynamics of metabolite production and microbiota composition and activity in dairy cows ${ }^{13,14}$. Measurements of $\mathrm{H}_{2}$ and $\mathrm{CH}_{4}$ as indicators of human gut microbial activity in vivo have been used before ${ }^{15-19}$, but these are in fact single-time-point gas measurements that lack the information that continuous analysis can provide.

Therefore, our study objective was to apply a simple non-invasive method to monitor the effect of diet on intestinal microbiota in real time using a human-relevant model, which we envisioned as a powerful tool to better understand the direct impact of nutrition on the microbiota and by extension of diet-microbiota interactions on human health.

C57BL/6] mice are the most widely used model in medical and nutritional health research and have shown their validity in dissecting microbe-host interactions and causality testing. 
However, analysis of fermentation gases in mice and other rodent models is a largely unexplored area. As is the case in humans, single-time-point measurements of $\mathrm{H}_{2}$ (ref. 2023) and $\mathrm{CH}_{4}$ (ref. 24-26) have been reported for mice and rats. This is critical, because only continuous measurements allow to faithfully study the time-resolved kinetics of digestion and metabolism of nutrients reaching the gut microbiota.

Indirect calorimetry makes use of the measurement of oxygen $\left(\mathrm{O}_{2}\right)$ and carbon dioxide $\left(\mathrm{CO}_{2}\right)$, as well as food and water intake and locomotor activity, to analyse energy metabolism. We have equipped a commercially available indirect calorimetry system with sensors for $\mathrm{H}_{2}$ and $\mathrm{CH}_{4}$, allowing continuous measurements of release of these gases non-invasively in real time. We applied this extended system to explore the adaptation of gut microbiota to highly- and lowly-digestible carbohydrates. To the best of our knowledge, this is the first time that food-microbiota interactions have been studied continuously, non-invasively and in real time in a murine model.

\section{Materials and Methods}

Coupling of hydrogen $\left(\mathrm{H}_{2}\right)$ and methane $\left(\mathrm{CH}_{4}\right)$ sensors into indirect calorimetry system

A PhenoMaster indirect calorimetry system (TSE Systems, Bad Homburg, Germany) was extended by coupling a Sensepoint $X C D \mathrm{H}_{2}$ gas analyser (Honeywell Analytics, Hegnau, Switzerland) and $\mathrm{CH}_{4}$ gas analyser (ABB Automation $\mathrm{GmbH}$, Frankfurt am Main, Germany) in a closed circuit in series in front of a Siemens High-Speed Sensor Unit containing the $\mathrm{O}_{2}$ and $\mathrm{CO}_{2}$ sensors. This order was chosen to prevent dilution of the sample with reference air, which is required by the Siemens unit. The $\mathrm{H}_{2}$ sensor has a stability of $< \pm 2 \%$ full scale deflection (fsd)/yr representing $<2 \mathrm{ppm} / \mathrm{yr}$ as it was adjusted to a measuring range from 0 to $100 \mathrm{ppm}$. The $\mathrm{CH}_{4}$ sensor has a zero drift of $\leq 1 \%$ of span per week and a measuring range from 0 to $500 \mathrm{ppm}$. A two point calibration of both $\mathrm{H}_{2}$ and $\mathrm{CH}_{4}$ analysers was performed within $24 \mathrm{~h}$ before each animal experiment. The calibration procedure was carried out using three gas mixtures (Linde Gas Benelux BV, Dieren, The Netherlands): zero (20.947\% $\mathrm{O}_{2}$ and $\left.\mathrm{N}_{2}\right)$, span $\mathrm{H}_{2}\left(98.8 \mathrm{ppm} \mathrm{H}_{2}\right.$ and synthetic air), and span $\mathrm{CH}_{4}(0.521 \%$ $\mathrm{CO}_{2}, 450 \mathrm{ppm} \mathrm{CH}_{4}$, and $\mathrm{N}_{2}$ ). The zero calibration mixture was flushed through the system for 10 min and $A D C$ signals were assigned $\mathrm{H}_{2}$ and $\mathrm{CH}_{4}$ values of $0 \mathrm{ppm}$. Thereafter, each of the span gas mixtures was run for $10 \mathrm{~min}$ and $A D C$ signals assigned $98.8 \mathrm{ppm}_{2}$ and $450 \mathrm{ppm} \mathrm{CH}_{4}$, accordingly. For $\mathrm{O}_{2}$ and $\mathrm{CO}_{2}$ calibration, the routine indicated in the TSE manufacturer's manual was followed, using an additional gas mixture $\left(0.999 \% \mathrm{CO}_{2}\right.$ and $\mathrm{N}_{2}$ ) for the span calibration point. Animals were measured as previously described ${ }^{27}$ with minor adjustments for the newly coupled sensors. These include the adjustment of airflow to $0.431 \mathrm{~min}^{-1}$ and the measuring time per cage set to $1.5 \mathrm{~min}$. Data was recorded using an updated, customized, version of the TSE PhenoMaster software (V5.8.0) specially developed for the incorporation of $\mathrm{H}_{2}$ and $\mathrm{CH}_{4}$ measurements.

Animal experiments and sample haruest

All animal experiments were approved by the Animal Ethical Committee (DEC 2014085.h) and performed in accordance to EU directive 2010/63/EU. Female and male 
C57BL/6]RccHsd mice (Harlan Laboratories BV, Horst, The Netherlands) were housed in Makrolon II cages enriched with wood chips and wood shavings, with free access to drinking water, at $23^{\circ} \mathrm{C} \pm 1{ }^{\circ} \mathrm{C}$ and a $12: 12 \mathrm{~h}$ light:dark cycle. Standard rodent chow (RMH-B, AB Diets, Woerden, The Netherlands) was provided exclusively and continuously since weaning, unless specified. Three different studies were conducted to investigate diethost-microbiota interactions upon provision of diets containing starches with differences in digestibility (the experimenter was not blinded to the diets that the animals were given).

Study 1 (long-term exposure, post-weaning): Mice were mated and the offspring reassigned to a foster dam 1 or 2 days after birth to obtain standardized litters. Males and females were stratified by body weight at post-natal day (PN) 21, housed individually and randomly assigned to be fed a highly- or a lowly-digestible starch diet (HDD and LDD, respectively; see below). The randomisation was achieved by generating a column of random numbers in a spreadsheet and sorting each diet and animal number according to the column of random numbers from smallest to largest. From PN36-42, a subgroup of mice was measured in the indirect calorimetry system with ad libitum access to the experimental diets (males: $n=12$ per diet, females HDD $n=12$, LDD $n=11)$. Fresh faecal pellets were sampled on PN39 ( $n=6$ per diet and sex) and stored at $-80{ }^{\circ} \mathrm{C}$ for intestinal microbiota analysis. Another subgroup of female mice was culled on PN42 for collection of caecum $(n=6)$ and colon contents ( $n=5$ HDD, $n=7$ LDD), and the faeces produced during the last week before sacrifice were collected for gross energy measurements (see In vivo diet digestibility). Before sacrifice, food was removed $1 \mathrm{~h}$ after the start of the light phase and animals decapitated 2-6 $\mathrm{h}$ after removal of food. Caecum and colon contents were immediately frozen in liquid nitrogen, and stored at $-80^{\circ} \mathrm{C}$ until analysis.

Study 2 (short-term exposure with fasting, adult): Eight-month-old female mice were individually housed in indirect calorimetry cages. After a 2-day adaptation period, mice were allowed a restricted amount $(1.1 \mathrm{~g})$ of chow $1 \mathrm{~h}$ before the onset of the dark phase to induce a fasting state in early morning, as published ${ }^{28}$. At the end of the light phase at $18.00 \mathrm{~h}$, mice were re-fed with a restricted amount $(1.1 \mathrm{~g})$ of chow, or first-time HDD or LDD (the refeeding diet was assigned randomly; $n=4$ per dietary group). Shortly before the following dark phase mice received access to the same diet they were allocated the day before, but now ad libitum. Indirect calorimetry measurements continued for an additional $5.5 \mathrm{~d}$.

Study 3 (short-term exposure without fasting, adult): Ten-month-old female mice were individually housed in indirect calorimetry cages. After a 2-day adaptation period, mice were provided clean bedding and given ad libitum first-time access to either HDD or LDD (random assignment, $n=6$ ) shortly before the dark phase and for the remaining experimental period. Measurements continued for an additional $4.5 \mathrm{~d}$. Faecal pellets produced after the introduction of the new diets were collected from the bedding at the end of the experiment and stored at $-80{ }^{\circ} \mathrm{C}$. 


\section{Experimental diets}

Both the HDD and the LDD satisfy the nutrient requirements for rodent growth and lactation (AIN-93G) ${ }^{29}$, with appropriate levels of mono- and poly-unsaturated fatty acids. The macronutrient composition was 20.1 energy percentage (en\%) protein, 54.9 en\% carbohydrates, and 25 en\% fat (Table 1), with starch being the sole source of carbohydrates. The starch fraction (Cargill BV, Sas van Gent, The Netherlands) of the HDD was composed of $100 \%$ amylopectin (which is highly digestible), while that of the LDD was a mixture of $60 \%$ amylose (which resists complete digestion) and $40 \%$ amylopectin. The diets were pelleted by Research Diet Services BV, Wijk bij Duurstede, The Netherlands.

Table 1. Diet composition

\begin{tabular}{|c|c|c|}
\hline \multirow{2}{*}{ Component } & \multicolumn{2}{|c|}{ Diet } \\
\hline & HDD & LDD \\
\hline Casein & 212.2 & 212.2 \\
\hline L-Cysteine & 3.0 & 3.0 \\
\hline Amylose mix (AmyloGel 03003) & 0.0 & 568.6 \\
\hline Amylopectin $\left(\mathrm{C}^{*} \mathrm{Gel} 04201\right)$ & 568.6 & 0.0 \\
\hline Coconut oil & 21.4 & 21.4 \\
\hline Sunflower oil & 83.1 & 83.1 \\
\hline Flaxseed oil & 14.2 & 14.2 \\
\hline Cholesterol & 0.03 & 0.03 \\
\hline Cellulose & 50.0 & 50.0 \\
\hline Mineral mix (AIN-93G-MX) & 35.0 & 35.0 \\
\hline Vitamin mix (AIN-93-VX) & 10.0 & 10.0 \\
\hline Choline bitartrate & 2.5 & 2.5 \\
\hline Total $(\mathrm{g})$ & 1000.0 & 1000.0 \\
\hline Gross energy density $\left(\mathrm{kJ} \mathrm{g}^{-1}\right)^{\mathrm{a}}$ & 18.9 & 19.5 \\
\hline Calculated energy density $\left(\mathrm{kJ} \mathrm{g}^{-1}\right)^{\mathrm{b}}$ & 17.9 & 17.9 \\
\hline Protein $(\mathrm{en} \%)^{\mathrm{b}}$ & 20 & 20 \\
\hline Carbohydrate $(\mathrm{en} \%)^{\mathrm{b}}$ & 55 & 55 \\
\hline Fat $(\mathrm{en} \%)^{\mathrm{b}}$ & 25 & 25 \\
\hline
\end{tabular}

In vivo diet digestibility

Total faeces produced from PN 35-42 (Study 1) were recovered from the bedding of a subgroup of randomly selected animals ( $n=4$ per sex and diet). Food intake was recorded over the same period. Gross energy in faeces and food was determined in blinded samples 
using a C7000 bomb calorimeter (IKA, Staufen, Germany) and diet digestibility was calculated as published ${ }^{30}$.

In vitro carbohydrate digestibility

The in vitro digestibility of starches in the experimental diets was determined in blinded samples in triplicate ${ }^{31}$. Briefly, 5 intact pellets of each diet were cryoground to homogeneous particle size and weighed separately into 3 tubes $(70 \mathrm{mg})$. Each sample was digested in a 15- $\mathrm{ml}$ tube by adding cocktail solutions (modified from Versantvoort et al. ${ }^{32}$ ) and digestive enzymes at $37^{\circ} \mathrm{C}$ in three sequential steps to represent the oral (5 min), gastric ( $2 \mathrm{~h}$ ), and duodenal $(6 \mathrm{~h})$ phases. Two blanks containing only enzymes and solutions were included. Samples were taken at several time points during the gastric and duodenal phases and centrifuged. Clean supernatants were recovered and free glucose content was determined by the glucose oxidase peroxidase method ${ }^{33}$. Starch digestion was expressed as the percentage of total glucose released based on the amount of starches in the diets.

Quantification of SCFA in intestinal digesta by gas chromatography (GC)

Short-chain fatty acids in caecum- and colon-contents were determined as previously reported $^{34}$, with some modifications. Samples (about $25 \mathrm{mg}$ ) were weighed, thawed, homogenized in $100 \mu \mathrm{l}$ of ultrapure water, and centrifuged for $3 \mathrm{~min}$ at 21,382 g. To $50 \mu \mathrm{l}$ of supernatant, $100 \mu \mathrm{l}$ of 2 -ethylbutyric acid solution $\left(0.45 \mathrm{mg} \mathrm{ml}^{-1}\right)$ were added as internal standard. An external standard curve was prepared containing $50 \mu \mathrm{l}$ of a mixture of acetic, propionic, butyric, valeric, isobutyric, and isovaleric acid at concentrations ranging from $0.002 \mathrm{mg} \mathrm{ml}^{-1}$ to $0.8 \mathrm{mg} \mathrm{ml}^{-1}$, to which $100 \mu \mathrm{l}$ of internal standard were added. Blanks containing only water or water and internal standard were included for quality control. $\mathrm{HCl}$ and oxalic acid were added to all samples, blanks, and standards in order to protonate the SCFA. Gas chromatography was performed on a FOCUS GC apparatus coupled to a flame ionization detector (Interscience, Breda, The Netherlands). Samples were injected $(1 \mu \mathrm{l})$ into an CP-FFAP CB column $(25 \mathrm{~m} \times 0.53 \mathrm{~mm} \times 1.00 \mu \mathrm{m}$; Agilent Technologies, Santa Clara, CA, USA). Helium served as carrier gas at a pressure of $40 \mathrm{kPa}$. The initial oven temperature was $100{ }^{\circ} \mathrm{C}$ with $0.5 \mathrm{~min}$ hold, ramped to $180{ }^{\circ} \mathrm{C}$ at $8{ }^{\circ} \mathrm{C} \mathrm{min}{ }^{-1}$ with $1 \mathrm{~min}$ hold,

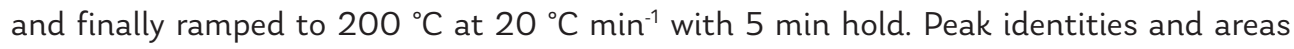
were analysed with Xcalibur software (version 2.2; Thermo Scientific, Waltham, MA, USA). Concentrations of SCFA were normalised to the internal standard and expressed relative to original sample weight.

\section{Microbiota analysis}

Microbial DNA was isolated from faecal pellets using the Maxwell ${ }^{\circledR} 16$ Instrument (Promega, Leiden, The Netherlands). Faecal pellets were added to a bead-beating tube with $350 \mu$ Stool Transport and Recovery (STAR) buffer, $0.25 \mathrm{~g}$ of sterilized zirconia beads $(0.1 \mathrm{~mm})$, and three glass beads $(2.5 \mathrm{~mm})$. Faecal pellets were homogenized by bead-beating three times $\left(60 \mathrm{~s} \times 5.5 \mathrm{~ms}\right.$ ) and incubation for $15 \mathrm{~min}$ at $95^{\circ} \mathrm{C}$ at $100 \mathrm{rpm}$. Samples were then centrifuged for $5 \mathrm{~min}$ at $4{ }^{\circ} \mathrm{C}$ and $14,000 \mathrm{~g}$ and supernatants transferred to sterile tubes. Pellets were re-processed using $200 \mu \mathrm{L}$ STAR buffer and both supernatants were pooled. 
DNA purification was performed with a customized kit (AS1220; Promega) using $250 \mu \mathrm{l}$ of the final supernatant pool. DNA was eluted in $50 \mu \mathrm{l}$ of DNAse- RNAse-free water and its concentration measured using a DS-11 FX+ Spectrophotometer/Fluorometer (DeNovix Inc., Wilmington, USA). The V4 region of 16S ribosomal RNA (rRNA) gene was amplified in duplicate PCR reactions for each sample in a total reaction volume of $50 \mu$ l. The master mix contained $1 \mu \mathrm{l}$ of a unique barcoded primer, 515F-n and 806R-n (10 $\mu \mathrm{M}$ each per reaction), $1 \mu \mathrm{l}$ dNTPs mixture, $0.5 \mu \mathrm{l}$ Phusion Green Hot Start II High-Fidelity DNA Polymerase (2 U/ $\mu$ l; Thermo Scientific, Landsmeer, The Netherlands), $10 \mu \mathrm{l} 5 \times$ Phusion Green HF Buffer, and $36.5 \mu \mathrm{l}$ DNAse- RNAse-free water. The amplification program included $30 \mathrm{~s}$ of initial denaturation step at $98^{\circ} \mathrm{C}$, followed by 25 cycles of denaturation at $98^{\circ} \mathrm{C}$ for $10 \mathrm{~s}$, annealing at $50{ }^{\circ} \mathrm{C}$ for $10 \mathrm{~s}$, elongation at $72{ }^{\circ} \mathrm{C}$ for $10 \mathrm{~s}$, and a final extension step at $72{ }^{\circ} \mathrm{C}$ for $7 \mathrm{~min}$. The PCR product was visualised in 1\% agarose gel ( $290 \mathrm{bp})$ and purified with CleanPCR kit (CleanNA, Alphen aan den Rijn, The Netherlands). The concentration of the purified PCR product was measured with Qubit dsDNA BR Assay Kit (Invitrogen, Caliornia, USA) and 200 ng of microbial DNA from each sample were pooled for the creation of the final amplicon library which was sequenced (150bp, paired-end) on the Illumina HiSeq2000 platform (GATC Biotech, Constance, Germany).

\section{Microbiota data processing and analysis}

Data filtering and taxonomy assignment were performed using the NG-Tax pipeline ${ }^{35}$. Briefly, an OTU table was created for each sample with the most abundant sequences. Low abundance OTU's were discarded, using a minimum relative abundance threshold of $0.1 \%$. Two distinct in-house assembled mock communities were included in the library and were compared with their theoretical composition for quality control (Fig. S1). Calculations for $\alpha$ and $\beta$-diversity analyses were performed using the publicly available Microbiome $R$ package (version 1.2.1) ${ }^{36}$. Adonis permutational multivariate analysis of variance (PERMANOVA) using either the weighted or unweighted Unifrac distances were performed with the Vegan package (version 2.5-2) and were used to determine the amount of variation explained by the grouping variables. Linear Discriminant Analysis (LDA) Effect Size (LEfSe) was applied to determine the differences between the microbial communities of HDD- and LDD-fed mice using a publicly available pipeline (http://huttenhower.sph.harvard.edu/galaxy/)37; the threshold for the logarithmic LDA score was set to 2.0. P values for Kruskal-Wallis and Wilcoxon tests for the LEfSe analysis were set to 0.05. For non-parametric Student's $t$-tests, reads were transformed to their relative abundances and tests were carried out with 999 permutations using QIIME (version 1; http://qiime.org/index.html) ${ }^{38}$. Statistical significance was determined using the Benjamini-Hochberg false discovery rate (FDR) adjustment.

\section{Data analysis}

Statistical analysis was performed using GraphPad Prism 5.04 (GraphPad, San Diego, CA, USA), unless stated otherwise. All data was tested for normality using the D'Agostino and Pearson omnibus test and its distribution was normalized by log transformation when applicable. Comparisons between two groups were made using unpaired two-tailed Student's t-tests (for data with normal distribution) or two-tailed Mann-Whitney $U$ tests 
$\left(\mathrm{VH}_{2}\right.$ during light phase between HDD and LDD). Comparisons between more than two groups were made by one-way analysis of variance (ANOVA) with post hoc Bonferroni's test for multiple comparisons. Time course data $\left(\mathrm{H}_{2}\right.$ evolution) was analysed by repeated measures two-way ANOVA with Bonferroni's post hoc test. When sample sizes being compared were similar and relatively large $(n>5)$, similarity of variances was not taken into account. All data is reported as mean \pm s.d. Statistical significance was set at $5 \%$, with levels indicated as ${ }^{*} P \leq 0.05,{ }^{* *} P \leq 0.01,{ }^{* * *} P \leq 0.001$, and ${ }^{* * *} P<0.0001$. Sample size was not determined statistically as the effect size was unknown, but it was based on our previous results on the use of indirect calorimetry to assess metabolic flexibility ${ }^{28,39}$.

\section{Results}

In vitro reflects in vivo diet digestibility

To confirm the difference in digestibility of the two starches incorporated into our experimental diets (Table 1), an in vitro model that mimics food digestion for the oral, gastric and small intestinal phases was used. The lowly-digestible starch diet (LDD) showed a slower and $14 \%$ less complete carbohydrate digestion than the highly-digestible starch diet (HDD; Figure 1). In addition, we quantified food intake and faecal energy content in female and male mice habituated to the experimental diets (Table 2). Daily faecal mass was increased in all mice fed LDD, whereas faecal energy density was increased in LDD females only. LDD mice lost on average twice as much energy in faeces compared to HDD mice. With similar food and energy intake, the diet digestibility was 6\% lower in LDD us HDD fed mice (Table 2). Taken together, both in vitro and in vivo analyses showed a reduced digestibility of the LDD us HDD.

Table 2. Dietary in vivo digestibility of the experimental diets.

\begin{tabular}{ccccccc} 
& \multicolumn{3}{c}{ Females } & \multicolumn{2}{c}{ Males } \\
\hline & HDD & LDD & $P$ value & HDD & LDD & $P$ value \\
\hline Food intake $(\mathrm{g})$ & $2.53 \pm 0.05$ & $2.71 \pm 0.24$ & 0.1942 & $2.82 \pm 0.21$ & $2.86 \pm 0.37$ & 0.8489 \\
\hline $\begin{array}{c}\text { Gross energy } \\
\text { intake (kJ) }\end{array}$ & $48.01 \pm 0.92$ & $53.08 \pm 4.76$ & 0.0816 & $53.36 \pm 4.01$ & $55.88 \pm 7.18$ & 0.5634 \\
\hline Faeces weight (g) & $0.20 \pm 0.01$ & $0.41 \pm 0.06$ & $\mathbf{0 . 0 0 0 6}$ & $0.24 \pm 0.02$ & $0.45 \pm 0.05$ & $\mathbf{0 . 0 0 0 2}$ \\
\hline $\begin{array}{c}\text { Faeces gross } \\
\text { energy (kJ g })\end{array}$ & $15.48 \pm 0.26$ & $16.18 \pm 0.24$ & $\mathbf{0 . 0 0 7 2}$ & $15.94 \pm 0.27$ & $16.01 \pm 0.23$ & 0.7227 \\
\hline $\begin{array}{c}\text { Faeces energy } \\
\text { loss (kJ) }\end{array}$ & $3.10 \pm 0.12$ & $6.68 \pm 1.08$ & $\mathbf{0 . 0 0 0 6}$ & $3.74 \pm 0.22$ & $7.26 \pm 0.78$ & $\mathbf{0 . 0 0 0 1}$ \\
\hline $\begin{array}{c}\text { Digestible energy } \\
\text { intake (kJ) }\end{array}$ & $44.91 \pm 0.91$ & $46.40 \pm 3.70$ & 0.4645 & $49.63 \pm 3.80$ & $48.62 \pm 6.44$ & 0.7967 \\
\hline $\begin{array}{c}\text { Diet digestibility } \\
(\%)\end{array}$ & $93.6 \pm 0.2$ & $87.5 \pm 0.9$ & $<\mathbf{0 . 0 0 0 1}$ & $93.0 \pm 0.2$ & $87.0 \pm 0.6$ & $<\mathbf{0 . 0 0 0 1}$
\end{tabular}

Energy balance calculated over the third week of exposure to the diets in females and males ( $n=4$ per sex and diet) and expressed per day. Statistical comparisons by Student's t-test within sex. Data reported as mean \pm s.d. 


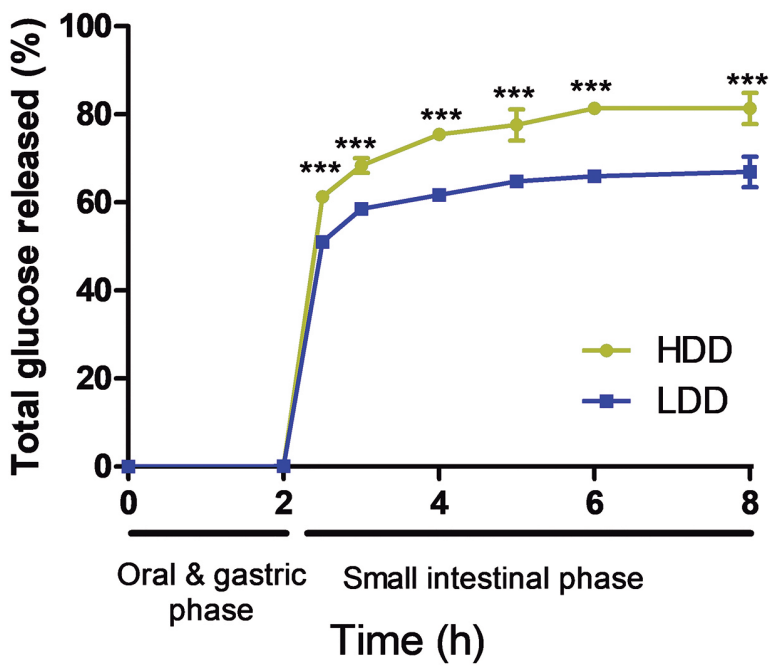

Figure 1. In vitro digestibility of starches in experimental diets. Triplicate samples of the lowly- and highlydigestible starch diets (LDD and HDD, respectively) were digested in vitro, and free glucose concentrations were determined at indicated time points. Statistical comparisons were made with two-way ANOVA with Bonferroni's post hoc test; ${ }^{* * *} P \leq 0.001$. Values are plotted as mean \pm s.d.

Measuring $\mathrm{H}_{2}$ production in real time

Reduced digestibility likely also affects colonic fermentation, for which $\mathrm{H}_{2}$ has been used as a marker in mice ${ }^{20}$. However, measurement of its continuous production in response to the diet has not yet been possible. We therefore adapted and extended an indirect calorimetry system to allow $\mathrm{H}_{2}$ and $\mathrm{CH}_{4}$ production to be studied in real time, by introducing the respective sensors in series with the $\mathrm{O}_{2}$ and $\mathrm{CO}_{2}$ sensors already present in the system (Fig. 2a). To determine if the small quantities of $\mathrm{H}_{2}$ originating from microbial carbohydrate fermentation in mice could be detected by our system, we measured gas concentrations in cages with and without chow-fed mice over $24 \mathrm{~h}$. Stable signals for all gases were seen in the absence of mice (Fig. 2b), and the concentrations were clearly decreased for $\mathrm{O}_{2}$ and increased for $\mathrm{CO}_{2}$ in mouse-occupied cages, as expected (Fig. 2c). $\mathrm{H}_{2}$ increased (Fig. 2c), while $\mathrm{CH}_{4}$ concentrations were not altered by the presence of a chow-fed mouse in the cage. The adapted indirect calorimetry system was therefore suitable for simultaneous respirometry and $\mathrm{H}_{2}$ production measurements in real time, however under the conditions tested, $\mathrm{CH}_{4}$ production appeared to be absent based on measured ambient levels well above the lower detection limit of the $\mathrm{CH}_{4}$ sensor (Fig. 2b).

$\mathrm{H}_{2}$ production indicates extent of carbohydrate digestibility

Since the contrasting digestibility of the experimental diets was expected to result in sustained differences in $\mathrm{H}_{2}$ production as a consequence of fermentation in the large intestine, we fed female and male mice, as a proof-of-concept, either the HDD or the LDD for three weeks and measured $\mathrm{H}_{2}, \mathrm{CH}_{4}, \mathrm{O}_{2}$, and $\mathrm{CO}_{2}$ levels continuously during several days (Study 1). Calculation of energy expenditure, based on $24 \mathrm{~h} \mathrm{O}_{2}$ consumption and $\mathrm{CO}_{2}$ 

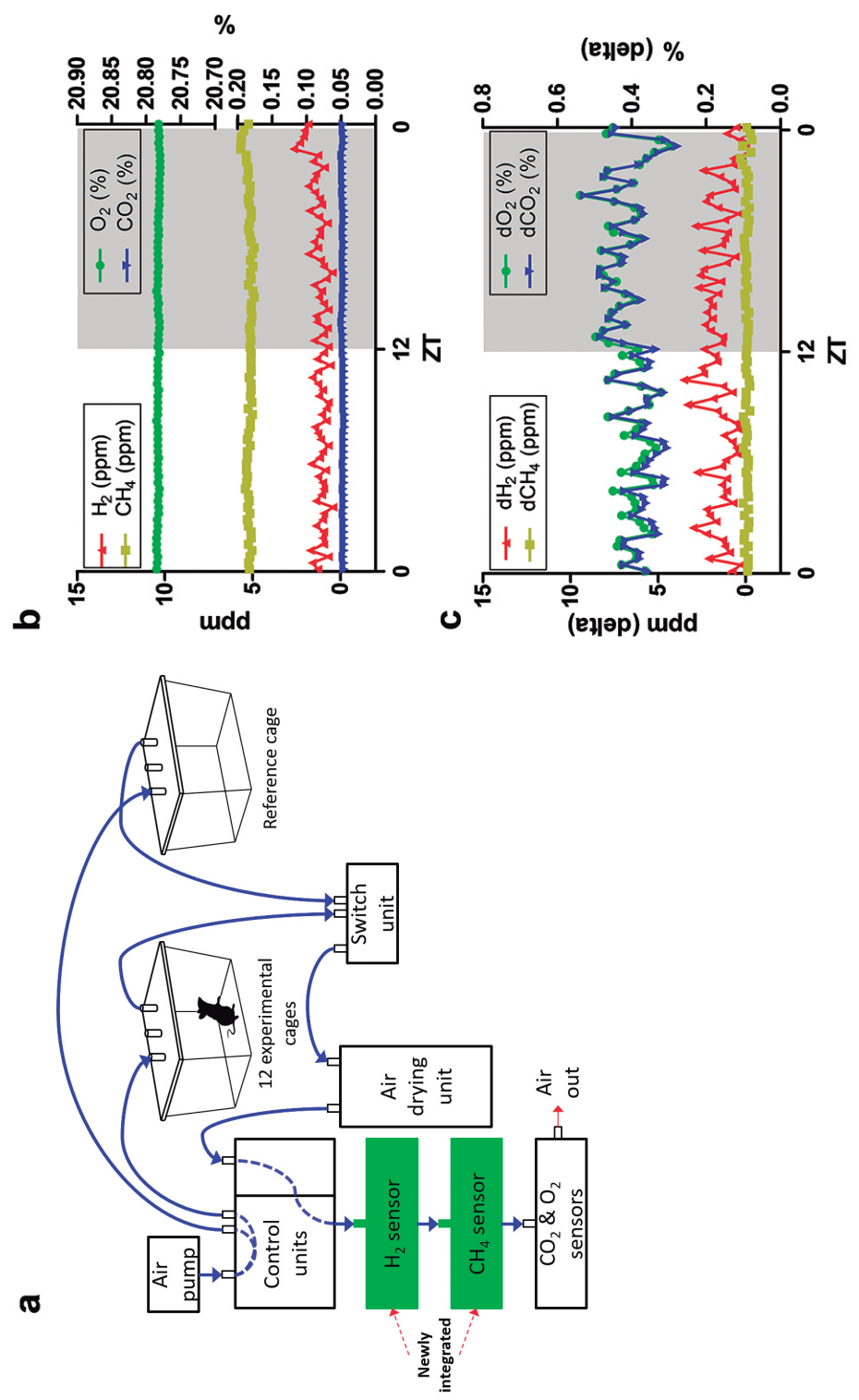

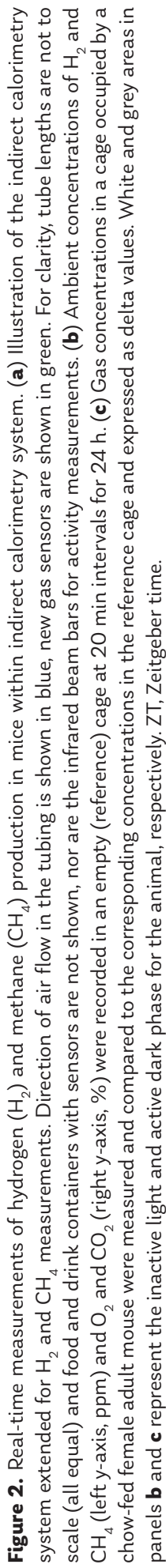


production, revealed no differences between dietary groups (females $1.59 \pm 0.08$ us $1.63 \pm$ $0.08 \mathrm{~kJ} \mathrm{~h}^{-1}$ in HDD and LDD respectively, $P=0.2094$; males $1.80 \pm 0.13$ us $1.76 \pm 0.13 \mathrm{~kJ} \mathrm{~h}^{-1}$ in HDD and LDD respectively, $P=0.5470)$. However, $24 \mathrm{~h}$ mean respiratory exchange ratio (RER) was lower in LDD- us HDD-fed male mice $(0.85 \pm 0.03$ us $0.88 \pm 0.03$ respectively, $P=0.0097$ ), indicating higher fat oxidation and lower carbohydrate oxidation in LDD mice. Overall, these observations agree with indirect calorimetry data reported for mice fed diets containing carbohydrates similar to the carbohydrates used here ${ }^{40}$.

Both LDD-fed females (Fig. 3a,b) and males (Fig. 3d,e) constantly produced more $\mathrm{H}_{2}$ than HDD-fed mice. A distinct pattern of $\mathrm{H}_{2}$ production became apparent in LDD-fed mice, with $\mathrm{H}_{2}$ levels being higher in the active dark phase and lower, but still clearly present, in the inactive light phase (Fig. 3a,b,d,e). This was fully consistent with the circadian food and starch intake (Fig. 3c,f). Importantly, the difference in $\mathrm{H}_{2}$ production between HDD- and LDD-fed mice was explained by the type of starch rather than the amount of starches ingested, as cumulative starch consumption was similar between the groups (Fig. 3c,f). Together, this data provides proof-of-concept for measuring $\mathrm{H}_{2}$ production in real time as an indicator of carbohydrate digestibility.

$\mathrm{H}_{2}$ evolution reflects adaptation to dietary carbohydrates

As we could show that $\mathrm{H}_{2}$ production can be sensitively and continuously measured, we next questioned whether it would be possible to measure adaptation to the diet in vivo in real time.

For this, we provided HDD or LDD to mice that had no previous exposure to these diets and we followed $\mathrm{H}_{2}$ production continuously. We introduced the new diets in one of two conditions; the first condition was as a single meal challenge given to fasted mice, followed by ad libitum access to the diet the next day, as a second fasting-refeeding challenge (Study 2, Fig. 4a). The second condition was by replacing the standard chow diet directly with HDD or LDD ad libitum (Study 3, Fig. 4b). $\mathrm{H}_{2}$ production was significantly increased in LDD- compared to HDD-fed mice as early as $4 \mathrm{~h}$ after fasted mice gained ad libitum access to the experimental diet (Fig. 4a). The direct switch from chow to HDD or LDD without fasting had similar results, with LDD-fed mice producing significantly more $\mathrm{H}_{2}$ after $53 \mathrm{~h}$ of access to the LDD compared to mice receiving HDD (2-way ANOVA, Fig. $4 b)$. In both conditions, i.e. fasted or directly switched to HDD or LDD, cumulative $\mathrm{H}_{2}$ production became significantly higher already within $12 \mathrm{~h}$ upon access to LDD us HDD (Fig. 4c,d), and $\mathrm{H}_{2}$ production patterns in LDD-fed mice closely followed the patterns of LDD intake (Fig. S2). Interestingly, mice that continued on the chow diet after a period of food restriction exhibited a spike in $\mathrm{H}_{2}$ production (Fig. $4 \mathrm{a}$ ), while consuming similar amounts of starches compared to the HDD and the LDD groups. $\mathrm{H}_{2}$ production in HDDfed mice remained lower compared to mice on LDD or chow, as expected. Importantly, LDD-induced $\mathrm{H}_{2}$ production increased gradually before reaching its maximal levels (up to $0.89 \mathrm{ml} \mathrm{h}^{-1}$ ), revealing the process of adaptation to the lowly-digestible starch. LDD- us HDD-fed mice thus showed a differential adaptation, likely in their microbiota, based on increased $\mathrm{H}_{2}$ production. 


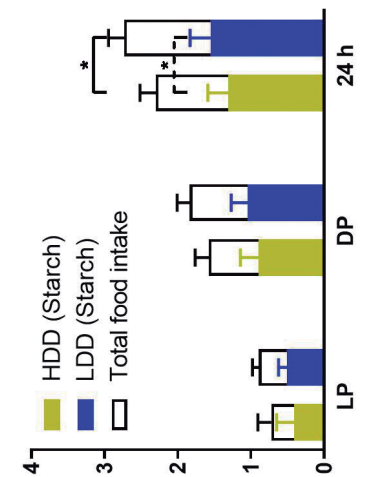

(6) әуеұи! әм!ฺำunว

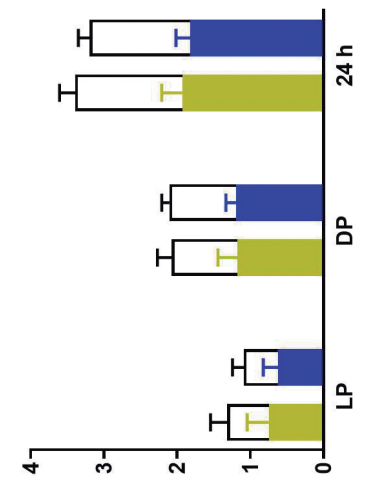

(6) әуеұน! әм!̨ำunว

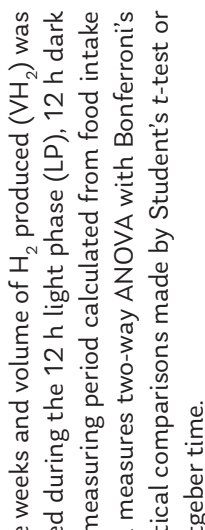

\&.

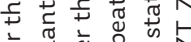

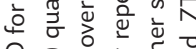

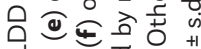

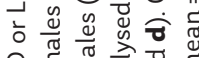

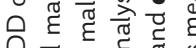

军政

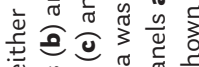

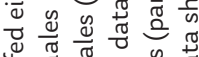

ष

ข้

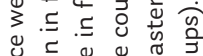

है

उ)

ฮั

E I $I^{N}$ 造 D

त.

తิ)

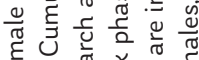

एँ हं क्ञ

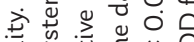

के 吾岳 $v$

苍

.0

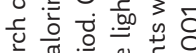

ㅎำ

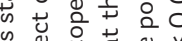

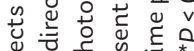

य

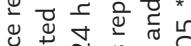

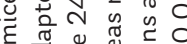

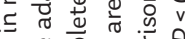

ᄃ वै ते

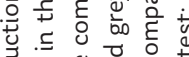

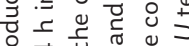

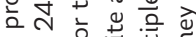

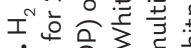

m

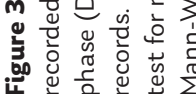

Non-Invasive Continuous Real-Time In Vivo Analysis of Microbial Hydrogen Production Shows Adaptation to Fermentable 
a
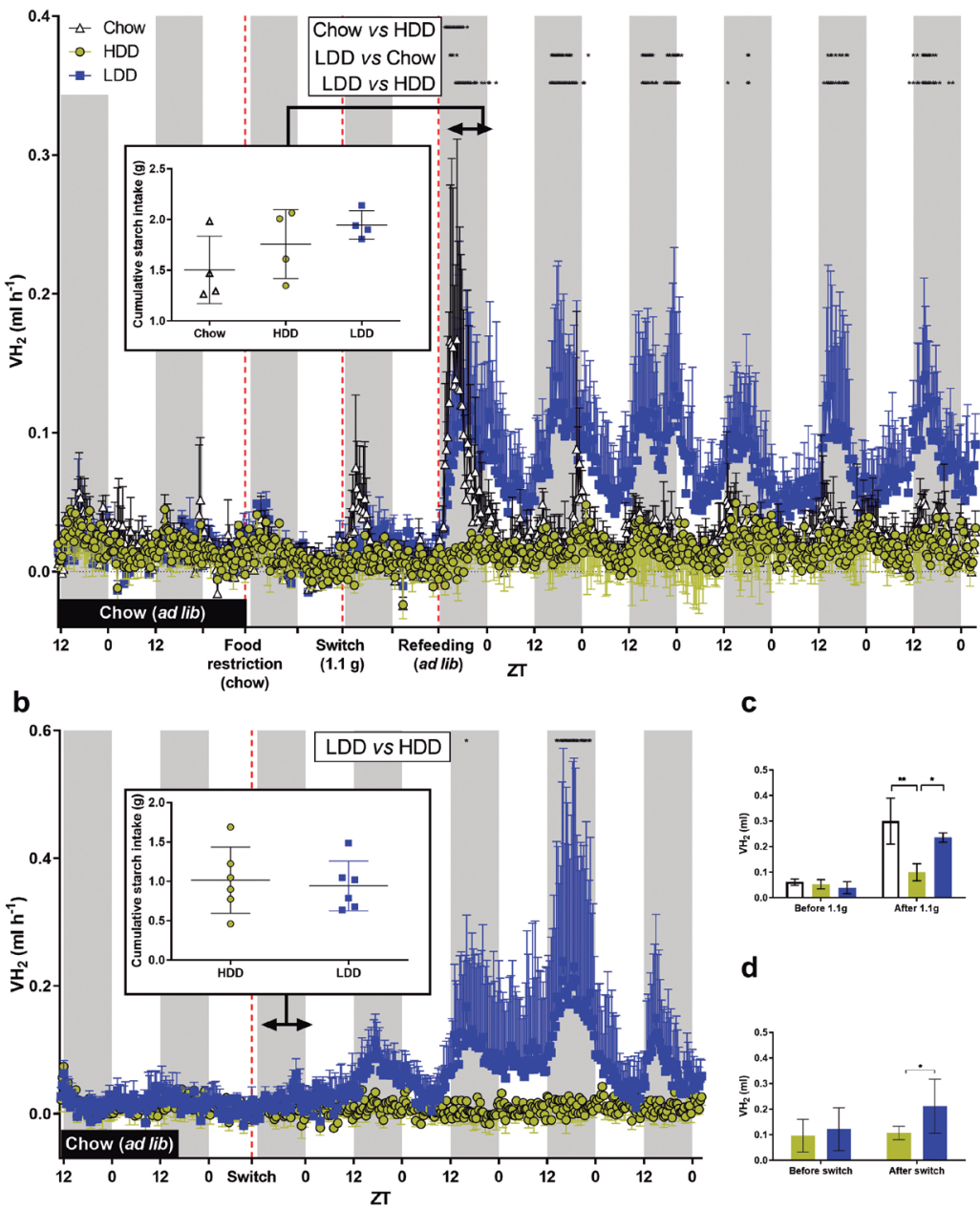

d

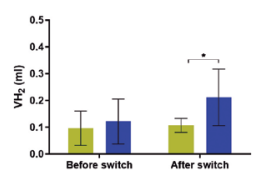

Figure 4. $\mathrm{H}_{2}$ evolution upon first exposure to starches of different digestibility. (a) Standard chow-fed mice within indirect calorimetry were food-restricted leading to fasting (dotted line), which was followed by feeding $1.1 \mathrm{~g}$ of chow (black), HDD (yellow), or LDD (blue; $n=4$ per group) prior to the dark phase as a single meal test ( $2^{\text {nd }}$ dotted line). As a result, they were fasted the next day, and received prior to dark phase ad libitum access to the same $\operatorname{diet}\left(3^{\text {rd }}\right.$ dotted line) for an additional 5.5 days. Inset: First $12 \mathrm{~h}$ cumulative starch-intake of ad libitum feeding with experimental diets. (b) Chow-fed mice ( $n=6$ per group) were switched to LDD or HDD without prior food restriction and measurements continued for another 4.5 days. Inset: First $12 \mathrm{~h}$ cumulative starch-intake after diet switch. (c) Cumulative $\mathrm{H}_{2}$ production over $12 \mathrm{~h}$ before (while food-restricted on chow) and after feeding $1.1 \mathrm{~g}$ of chow, HDD, or LDD ( $n=4$ per group). (d) Cumulative $\mathrm{H}_{2}$ production over $12 \mathrm{~h}$ before and after switching directly from chow to HDD or LDD ( $n=6$ per group). All mice received no other diet than chow during their whole lifetime prior to these experiments and the dietary switch (black bar), but colour usage reflects subgroups after first exposure to new diets. White and grey areas represent light and dark phases, respectively. Time course data was analysed by repeated measures two-way ANOVA with Bonferroni's test for multiple comparisons (chow vs HDD, LDD vs chow, and LDD vs HDD), and time points where $P<0.05$ are indicated with black stars (panels $\mathbf{a}$ and $\mathbf{b}$ ). Cumulative data was statistically compared using unpaired two-tailed Student's $t$-test (between HDD and LDD) and one-way ANOVA with Bonferroni's multiple comparisons post hoc test (between chow, HDD, and LDD); ${ }^{*} P \leq 0.05,{ }^{* *} P \leq 0.01$. Data is presented as mean \pm s.d. For clarity, either upper or lower error bars are shown. ZT, Zeitgeber time. 


\section{CHAPTER 2}

Alterations in intestinal microbiota by dietary carbohydrates

Since the production of $\mathrm{H}_{2}$ fully depends on intestinal microbial communities and their metabolism, we further investigated the changes in the microbiota induced by the LDD to validate our observations. As an additional parameter of fermentation, we first assessed SCFA levels in intestinal digesta after 3 weeks of exposure to the HDD or the LDD (Study 1). Total caecal SCFA levels were similar between LDD- and HDD-fed mice (35.6 13.9 us $34.9 \pm 11.9 \mu \mathrm{mol} \mathrm{g}^{-1}$, respectively), including valeric and isobutyric levels (data not shown), whereas total SCFA in colon were higher in LDD- compared to HDD-fed mice $(25.6 \pm 9.6$ us $9.6 \pm 4.1 \mu \mathrm{mol} \mathrm{g}{ }^{-1}, P=0.0059$ ). Acetic acid (Fig. 5a) and propionic acid (Fig. 5b) were the two most abundant SCFA, and both were significantly elevated in LDD-fed mice in colon and caecum contents, respectively. Butyric acid was the most differentially produced SCFA, enriched by 13.8-fold in LDD colon content (Fig. 5c). Finally, isovaleric acid, a product of microbial protein fermentation, was the least abundant of the measured SCFA in all groups and was significantly lower in caecum of LDD- us HDD-fed mice (Fig. 5d).

a
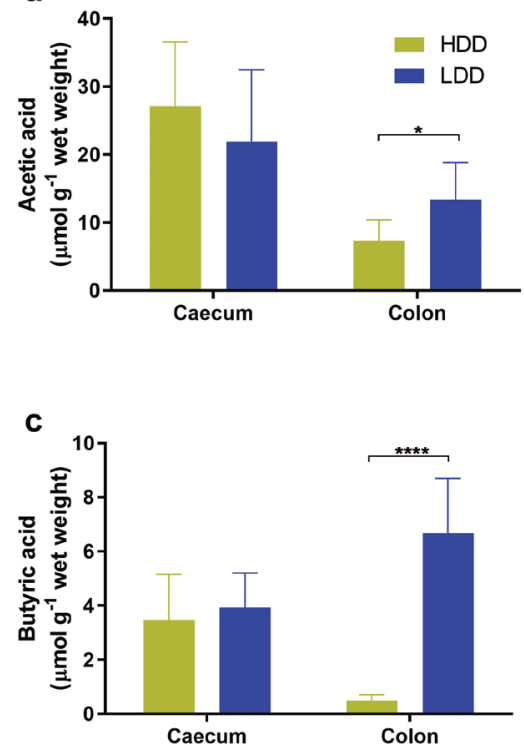

b

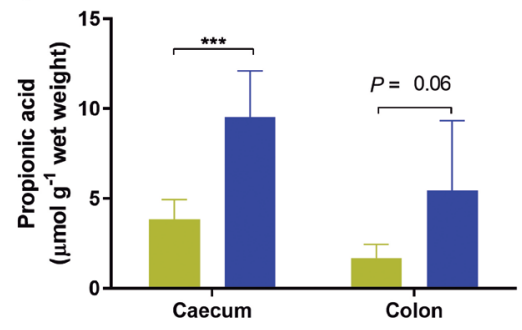

d

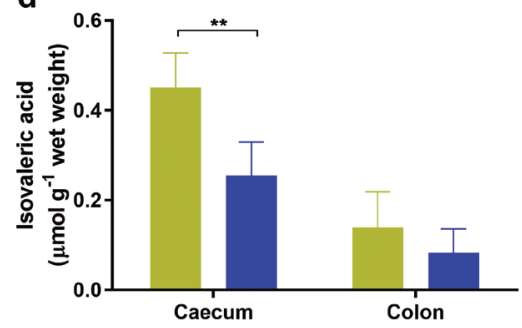

Figure 5. Short-chain fatty acids (SCFA) concentrations in intestinal digesta of mice fed starches of different digestibility. (a) Acetic acid, (b) propionic acid, (c) butyric acid and (d) isovaleric acid concentrations in mouse caecum ( $n=6$ per group) and colon ( $n=5 \mathrm{HDD}, n=7 \mathrm{LDD}$ ) contents obtained after three weeks of feeding HDD (yellow bars) or LDD (blue bars). Statistical comparisons were made using unpaired two-tailed Student's t-test; ${ }^{*} P \leq 0.05,{ }^{* *} P \leq 0.01,{ }^{* * *} P \leq 0.001,{ }^{* * *} P<0.0001$. Data shown as mean \pm s.d.

We next compared the overall changes in faecal microbiota communities induced by HDD or LDD after exposure to the diets for 3 weeks (Study 1) and $4.5 \mathrm{~d}$ (Study 3). Principal coordinates analysis (PCoA) using the UniFrac unweighted distance matrix revealed a clear separation between the two dietary groups (Fig. 6a). These observations were supported by Adonis analysis, using either weighted or unweighted UniFrac distances, as 
diet explained a large part of the variation in microbiota composition (20\% and 29\%, respectively, $P<0.001$, Table 3 ). $\mathrm{H}_{2}$ volume was the second most important variable, followed by duration of intervention and age, and body weight, with minor but significant effects (Table 3). Of note, duration of intervention and age of mice are dependent variables due to study design. In order to control for the effects of duration of dietary exposure, we also analysed Studies 1 and 3 separately. After 3 weeks of intervention, diet and $\mathrm{H}_{2}$ production were the only significant variables, with $\mathrm{H}_{2}$ explaining up to $34 \%$ of the variation (Fig. $6 \mathrm{~b}$ and Table 3). However, only diet contributed significantly to the variation after $4.5 \mathrm{~d}$ of intervention in adult mice (Fig. $6 \mathrm{c}$ and Table 3). Additionally, $\alpha$-diversity appeared to decrease with duration of intervention irrespective of the dietary intervention, with no consistent effects of the diet (Fig. S3). This is in line with the differences in age of these mice, namely the young mice showing lower $\alpha$-diversity.

We then aimed to identify which microbial taxa were significantly associated with the observed differences in $\beta$-diversity. The microbiota of mice fed LDD us HDD for 3 weeks was enriched in Bacteroides, Parasutterella, Roseburia, and Alloprevotella, along with two other families (Fig. 7a and Fig. S4a). In comparison, Lactobacillus, Rikenella, Odoribacter, Enterorhabdus, and Desulfovibrio among others appeared enriched in HDD us LDD-fed mice (Fig. 7a and Fig. S4b). Similar differences were seen after $4.5 \mathrm{~d}$ of exposure (Fig. 7b and Fig. S4c,d). While fewer taxa were affected by the short-term dietary intervention, changes in genus level were consistent for both groups (Fig. S5). Moreover, $\mathrm{H}_{2}$ production was the only (environmental) variable that was significantly correlated with specific bacteria taxa after three weeks of intervention, with five genera correlating positively with $\mathrm{H}_{2}$ production and eight genera showing a negative correlation (Fig. 8 and Fig. S6). Eleven of these 13 genera were also significantly influenced by diet (Fig. 7a and Fig. S4a,b). Finally, Archaea (some of which are $\mathrm{CH}_{4}$ producers) could not be detected in any of the samples despite the use of primers targeting both bacterial and archaeal 16S rRNA genes equally well. This agrees with the absence of $\mathrm{CH}_{4}$ detection in these mice and under these nutritional challenges.

\section{Discussion}

The goal of this study was to measure real-time interactions between diet, gut microbes, and the host. We implemented $\mathrm{H}_{2}$ and $\mathrm{CH}_{4}$ detection in an indirect calorimetry system to track fermentation continuously in mice. $\mathrm{H}_{2}$ production revealed a time frame for microbiota adaptation to starch of low digestibility, which corresponded with shifts in microbial community composition induced by diet. Thus, measuring $\mathrm{H}_{2}$ production allowed us to non-invasively study effects of the diet on the intestinal microbiota in real-time. The difference in starch digestibility as part of the experimental diets was confirmed both by in vitro and in vivo measurements, but did not significantly alter total intake of digestible energy (gross energy minus faecal energy losses) between dietary groups within a sex. The lower digestibility of the starch in the LDD thus suggests that partially undigested starch reached the large intestine which was subsequently partially fermented by the intestinal microbiota providing energy substrates, e.g. SCFA, to the host. Energy of undigested starch can be lost after fermentation in the form of products not utilizable by the host, such as $\mathrm{H}_{2}$. 
However, previous studies considered energy loss in the form of $\mathrm{H}_{2}$ and $\mathrm{CH}_{4}$ negligible, representing less than $0.2 \%$ of total energy expenditure in humans consuming nonstarch polysacharides ${ }^{41}$. Studies in rats fed various types of resistant starch also indicated that energy loss through fermentation is minimal, although the actual $\mathrm{H}_{2}$ output was not measured directly ${ }^{42}$. Here, our data show that $\mathrm{H}_{2}$ is produced constantly on a lowlydigestible starch diet. Although the volume of $\mathrm{H}_{2}$ produced by the mice in our study may be little in terms of energy loss, it is plausible that carbohydrates that give a higher level of fermentation could further increase the $\mathrm{H}_{2}$ output, which might represent a significant factor to take into account over a lifetime.

$\mathrm{H}_{2}$ production was detected in mice under all conditions tested, with the amounts produced clearly being influenced by the form of carbohydrate consumed. Mice fed moderately fermentable carbohydrates have been shown to produce $\mathrm{H}_{2}{ }^{20}$. Even in conditions where little fermentation is expected, such as feeding corn starch-based chow ${ }^{43}$ or pure sucrose ${ }^{44}$, $\mathrm{H}_{2}$ production has been seen in rats. In line with our data on three diets with a different carbohydrate profile, this illustrates that $\mathrm{H}_{2}$ production can directly reflect subtle changes in carbohydrate fermentation. Interestingly, $\mathrm{H}_{2}$ production was clearly associated with the food intake pattern. This is in contrast with data reported in humans, where $\mathrm{H}_{2}$ and $\mathrm{CH}_{4}$ peaked at rather unpredictable times after food intake despite the proper control of the meal schedule ${ }^{41}$. This might be due to e.g. differences in dietary meal composition, time resolution of sampling, intestinal transit time, or other differences in intestinal physiology between humans and mice. More recently, using gut capsule technology, a similar $\mathrm{H}_{2}$ pattern as in our mice was also observed in a human pilot trial based on dietary fibre differences ${ }^{45}$.

We demonstrated that real-time monitoring of $\mathrm{H}_{2}$ production can be used to investigate transient effects of diet in time and explore the process of adaptation rather than the end stage only. So far, studies mainly investigated, by measuring $\mathrm{H}_{2}$ and other fermentation parameters at selected time points, longer timeframes ranging from 1 day to several weeks ${ }^{25,46-48}$. In our study, significant differences in $\mathrm{H}_{2}$ production appeared within $12 \mathrm{~h}$ upon access to LDD. This timeframe was clearly influenced by fasting and whether the diet was provided ad libitum or in a restricted amount. We speculate these early increases in $\mathrm{H}_{2}$ output to reflect immediate effects of diet on microbial metabolism preceding changes in community structure. Another observation was that mice fed chow produced $\mathrm{H}_{2}$, although at low levels. Real-time monitoring newly revealed that a period of food restriction followed by refeeding led to a marked and acute increase in $\mathrm{H}_{2}$ production once chow became available again. A likely explanation is excessive eating after food deprivation, causing a larger amount of not fully digested chyme to enter the large intestine and thus increasing substrate availability to the microbiota. In addition, a $24 \mathrm{~h}$ fasting period alone has been shown to produce shifts in microbial diversity ${ }^{49}$ and microbiota configuration ${ }^{50}$. Such changes could in turn alter fermentation stoichiometry and microbial function in response to the diet and ultimately lead to a higher $\mathrm{H}_{2}$ output. Our analysis indicates (short-term) effects of fasting and refeeding on microbial activity, which should be careful taken into account in nutritional studies focussing on changes in microbiota composition and function. 
CHAPTER 2

Table 3. Faecal microbiota composition of mice fed HDD or LDD and other host and environmental variables.

\begin{tabular}{|c|c|c|c|c|}
\hline & \multicolumn{2}{|c|}{ Weighted UniFrac } & \multicolumn{2}{|c|}{ Unweighted UniFrac } \\
\hline & $\mathrm{R}^{2}$ & $P$ value & $\mathrm{R}^{2}$ & $P$ value \\
\hline & \multicolumn{4}{|c|}{ Studies 1 and 3 combined (long- and short-term exposures) } \\
\hline Diet & 0.198 & $0.001 * * *$ & 0.287 & $0.001 * * *$ \\
\hline Cumulative $\mathrm{H}_{2}$ production & 0.098 & $0.023 *$ & 0.173 & $0.001 * * *$ \\
\hline Duration of intervention* & 0.094 & $0.015^{*}$ & 0.141 & $0.001 * * *$ \\
\hline Age* & 0.094 & $0.02 *$ & 0.141 & $0.002 * *$ \\
\hline Sex & 0.057 & 0.12 & 0.05 & $0.018^{*}$ \\
\hline Body weight & 0.08 & $0.042 *$ & 0.1 & $0.002 * *$ \\
\hline Food intake & 0.06 & 0.08 & 0.043 & 0.142 \\
\hline Starch intake & 0.068 & 0.079 & 0.043 & 0.135 \\
\hline
\end{tabular}

Study 1 (long-term exposure, post-weaning, $n=12$ )

\begin{tabular}{lcccc}
\hline Diet & 0.26 & $0.003^{* * *}$ & 0.4 & $0.001^{* * *}$ \\
\hline Cumulative $\mathrm{H}_{2}$ production & 0.198 & $0.005^{* * *}$ & 0.344 & $0.001^{* * *}$ \\
\hline Sex & 0.062 & 0.195 & 0.03 & 0.658 \\
\hline Body weight & 0.046 & 0.289 & 0.02 & 0.87 \\
\hline Food intake & 0.099 & 0.062 & 0.049 & 0.3 \\
\hline Starch intake & 0.1 & 0.077 & 0.05 & 0.279 \\
\hline
\end{tabular}

Study 3 (short-term exposure, adult, $n=5$ )

\begin{tabular}{llllc}
\hline Diet & 0.24 & 0.056 & 0.293 & $0.004 * * *$ \\
\hline Cumulative $\mathrm{H}_{2}$ production & 0.14 & 0.217 & 0.108 & 0.5 \\
\hline Body weight & 0.08 & 0.594 & 0.137 & 0.228 \\
\hline Food intake & 0.06 & 0.748 & 0.192 & $0.044^{*}$ \\
\hline Starch intake & 0.06 & 0.761 & 0.192 & 0.05 \\
\hline
\end{tabular}

Results are obtained using Adonis Permutational Multivariate Analysis of Variance. * Duration of intervention (short- and long-term) and age of animals (young us adult) are not independent variables.

As could be expected, the driver of the experimental differences, the dietary starch digestibility, was the most important factor explaining the variation in microbiota, showing colinearity with our measured in vivo $\mathrm{H}_{2}$ production. Although current knowledge of the dynamics of $\mathrm{H}_{2}$ within the gastrointestinal tract is limited, it is well documented that $\mathrm{H}_{2}$ is exclusively produced during fermentation by hydrogenogens ${ }^{6}$. Among the major hydrogenogenic bacteria are Bacteroidetes and clostridial members of Firmicutes ${ }^{51}$. In line with this, we observed that LDD, a source of carbohydrates for caecum and colon, stimulated the fermentative Bacteroidetes bacteria, more specifically the genus Bacteroides. This is consistent with the dose-dependent increase in caecal Bacteroidetes density in response to amylose $\mathrm{e}^{52}$ and similar findings for amylose on a high-fat background ${ }^{53}$. Here we extend these findings and show, for the first time in vivo, a positive correlation between Bacteroides and $\mathrm{H}_{2}$ production in mice. 
a

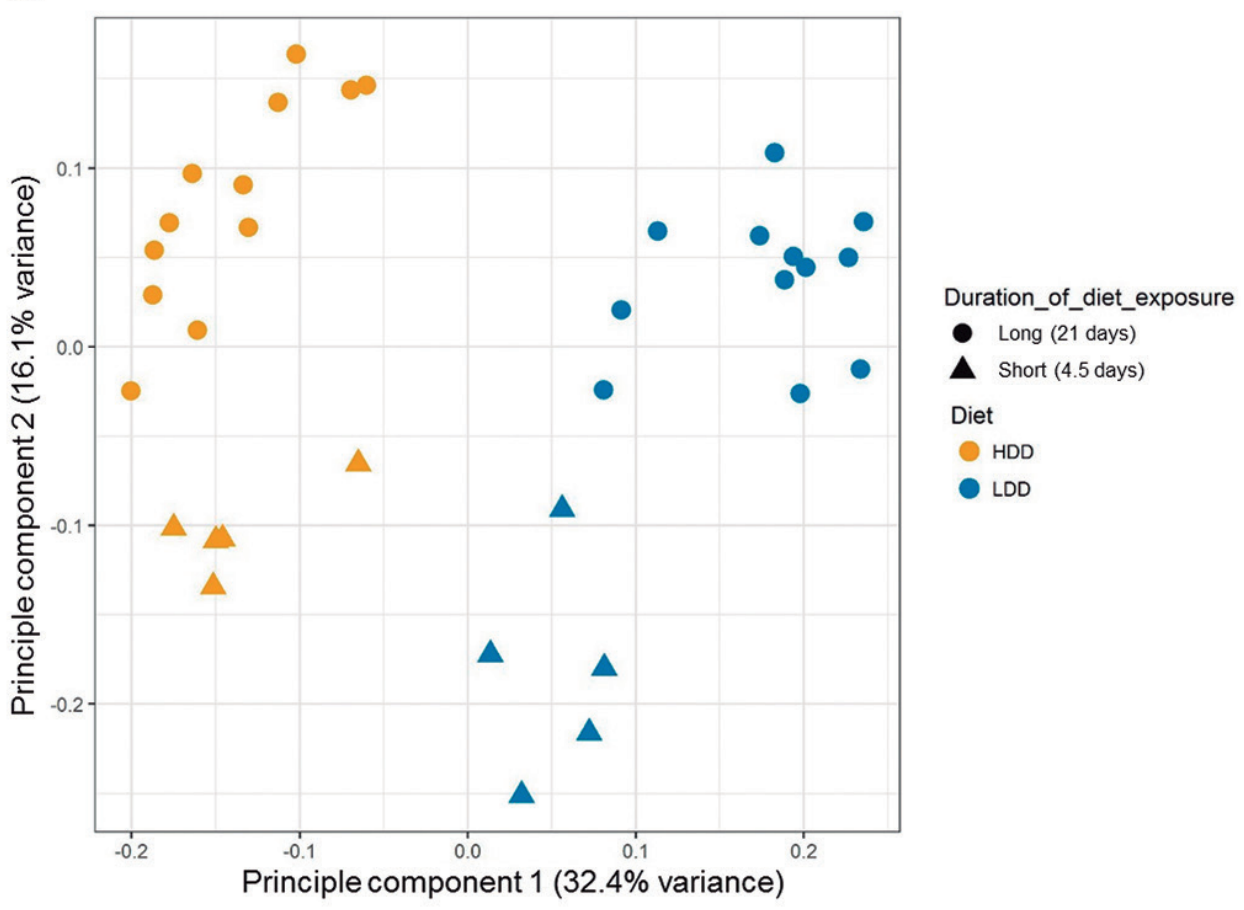

b

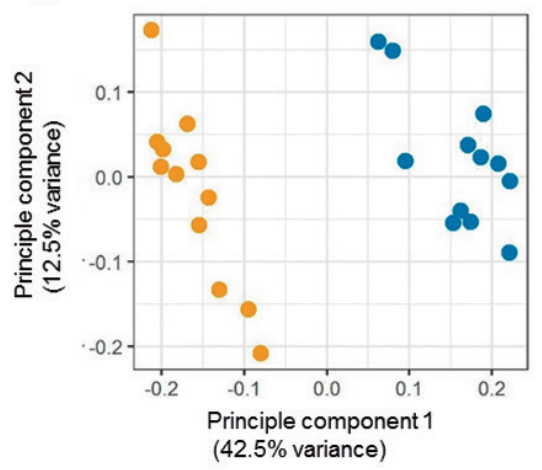

C

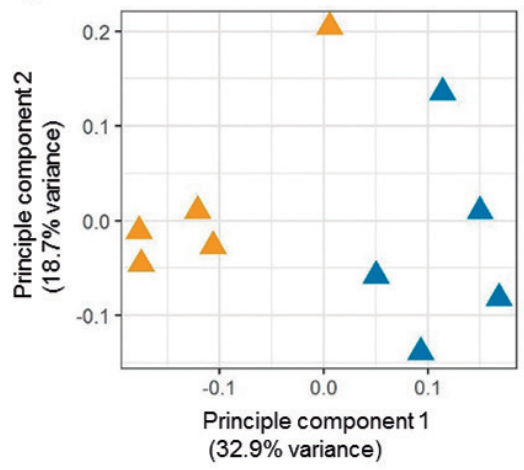

Figure 6. Starch digestibility primarily determines faecal microbiota composition. Principal coordinates analysis (PCoA) plot illustrating the unweighted UniFrac distances of the intestinal microbiota of mice after long-and short-term exposure to HDD and LDD (a, Studies 1 and 3 combined), and only long-term (b, Study 1 ) and shortterm (c, Study 3) exposures. Each data point represents a sample of faecal pellets of one individual mouse $(n=12$ long-term exposure per diet, $n=5$ short-term exposure per diet). 
a
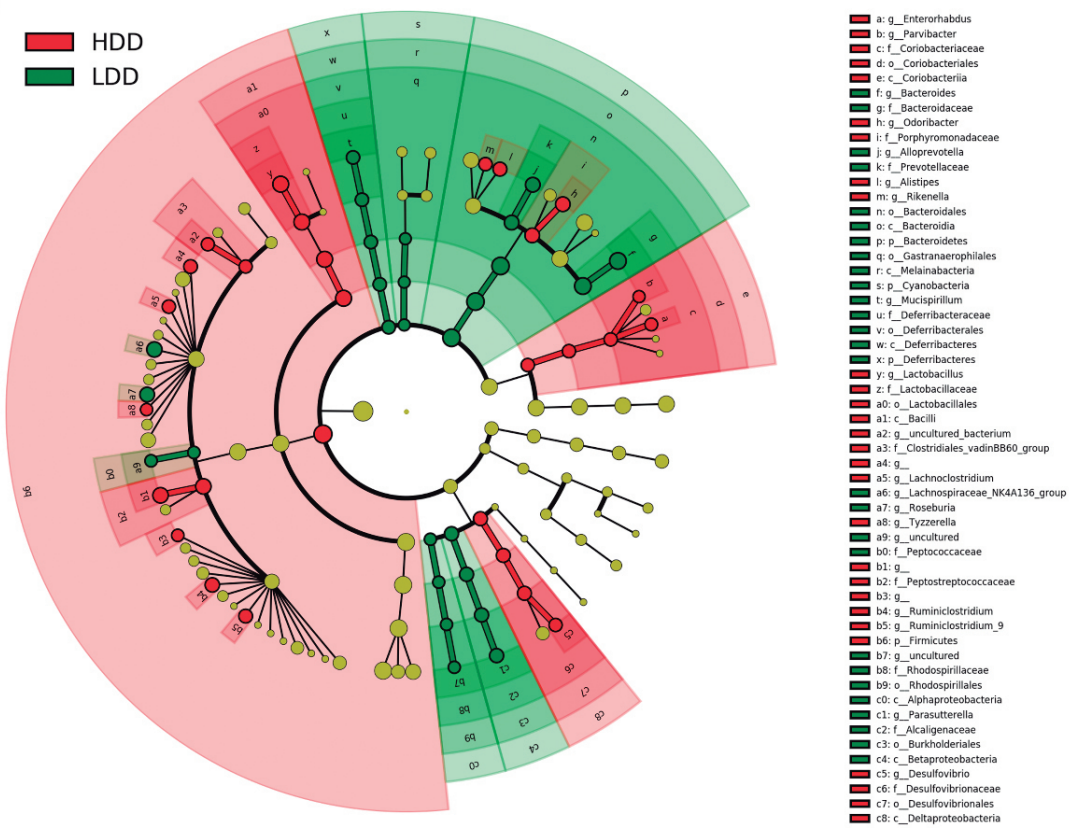

b
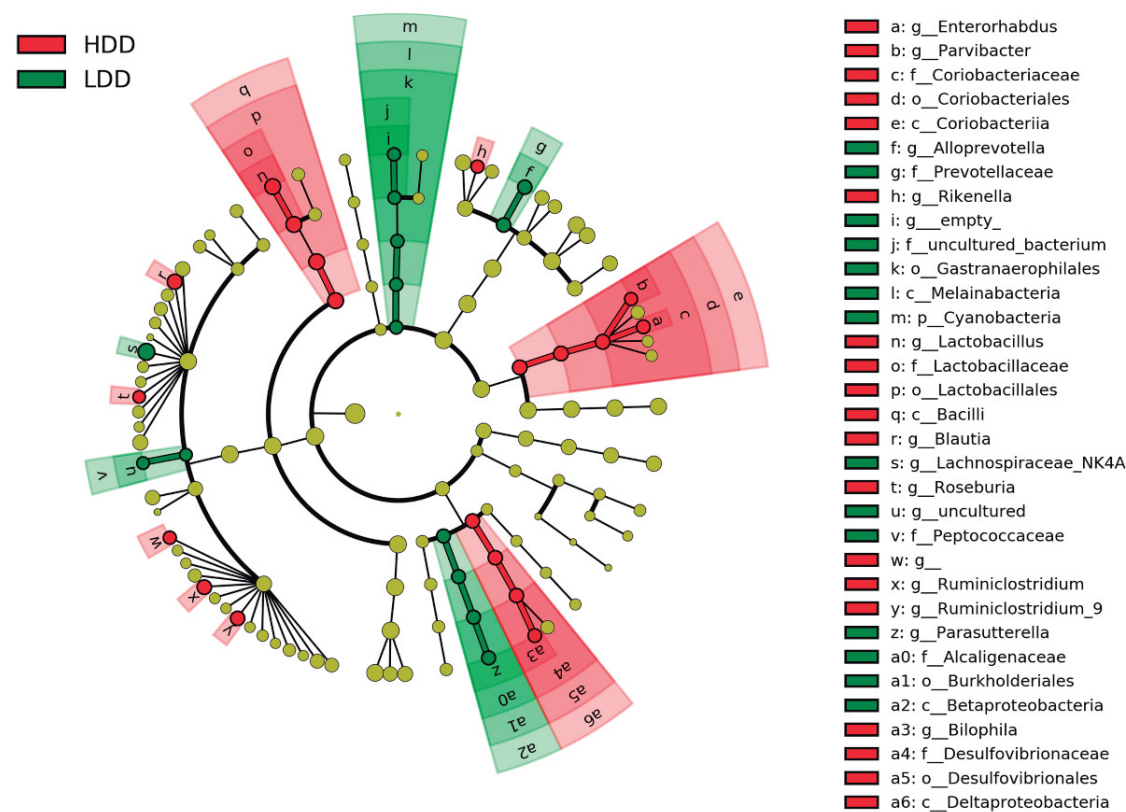

Figure 7. Exposure to starches of different digestibility induces distinct microbial taxa. (a) Cladogram representing bacteria genera that were significantly enriched by LDD or HDD after 3 weeks of exposure to the diets $(n=12$ per diet, Study 1). (b) Bacterial genera that were significantly increased by LDD or HDD after $4.5 \mathrm{~d}$ of exposure to the diets ( $n=5$ per diet, Study 3). Comparisons were done using the linear discriminant analysis effect size (LEfSe) method. LDA scores are shown in Fig. S4e,f. Nomenclature of microbial strains is based on phylum, class, order, family, and genus. 


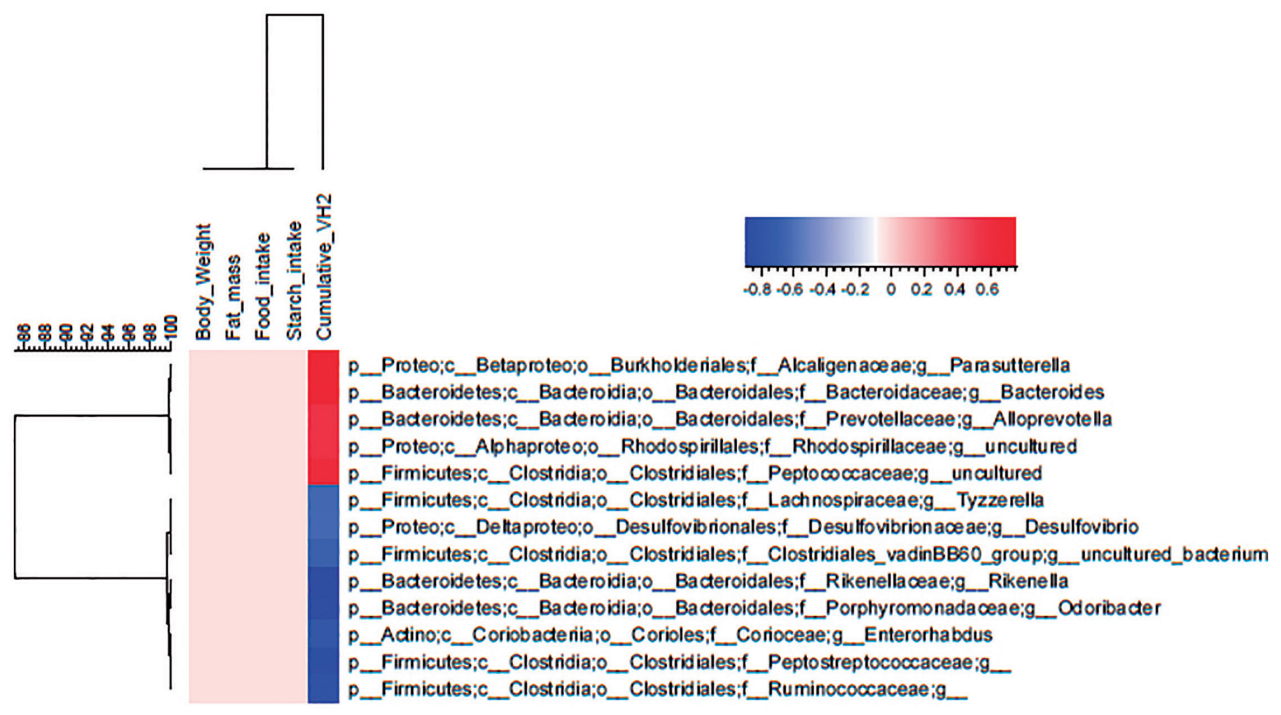

Figure 8. Specific bacterial genera correlate only with in vivo $\mathrm{H}_{2}$ production. Spearman's rank correlation coefficients of faecal microbiota, $\mathrm{H}_{2}$ production, food and starch intake, body weight, and fat mass of mice exposed to HDD or LDD for 3 weeks after weaning ( $n=12$ per diet, Study 1 ). Non-red and non-blue cells all have a Spearman's correlation value of 0 with FDR $P$ value $>0.13$. Nomenclature of microbial strains is based on phylum, class, order, family, and genus.

Interestingly, after the short-term exposure to LDD in adult mice, Bacteroidetes were not significantly increased compared to the HDD group. This might be associated with the shorter duration of the treatment and possibly with more firmly established microbial communities in adulthood. However, most genera induced by diet in adult mice after 4.5 days correspond to those induced in mice after weaning, which were exposed for 3 weeks.

Another consistent shift in microbial community composition was the promotion of Deltaproteobacteria, particularly Desulfovibrio and Bilophila, in HDD-fed mice. Deltaproteobacteria are the major representatives of colonic sulphate reducing bacteria $(\mathrm{SRB})^{54}$ including Desulfovibrio. SRB along with methanogens and acetogenic bacteria are the only gut microbes able to use $\mathrm{H}_{2}$ as an electron donor to produce $\mathrm{H}_{2} \mathrm{~S}$ and acetate. Although not a SRB itself, taurine-respiring Bilophila species can also produce $\mathrm{H}_{2} \mathrm{~S}$. Additionally, there is evidence of $\mathrm{CH}_{4}$ production in rats ${ }^{26}$ and mice, with the presence of methanogens in humanized microbiota mouse models $\mathrm{s}^{55}$ and by high fat dietary feeding ${ }^{56}$. The fact that we neither detected $\mathrm{CH}_{4}$ nor Archaea suggests that $\mathrm{H}_{2}$ was preferentially used to produce $\mathrm{H}_{2} \mathrm{~S}$ in mice fed readily digestible starch. $\mathrm{H}_{2} \mathrm{~S}$ is a potentially toxic product of bacterial metabolism ${ }^{57-59}$, and it has been implicated in human health and disease ${ }^{19}$ and, more recently, thermogenesis ${ }^{60}$. Moreover, $\mathrm{H}_{2} \mathrm{~S}$ has been reported to inhibit the production of SCFA and specifically to impair butyrate oxidation, depriving colonic cells from their main energy source ${ }^{58,61}$. In line, we report a dramatic difference in colonic butyrate in HDD-fed mice. Apart from Deltaproteobacteria, we observed increased abundances of Odoribacter, a known $\mathrm{H}_{2} \mathrm{~S}$ producer ${ }^{62}$, and Rikenella, a desulphatase-secreting bacterium ${ }^{63}$, under HDD- 
feeding. Members of the genus Rikenella are able to cleave sulphate from mucin glycans, making them available for microbial degradation ${ }^{64}$ and potentially acting as a donor of sulphate to $\mathrm{H}_{2} \mathrm{~S}$ producers. Based on these facts we speculate that the lack of fermentable carbohydrates favoured the presence of hydrogenotrophs associated with the production of $\mathrm{H}_{2} \mathrm{~S}$, which could have led to the decreased $\mathrm{H}_{2}$ output and colonic SCFA levels that was observed in mice fed highly-digestible starch.

The major taxon increased in HDD-fed mice in our study belonged to the genus Lactobacillus. In contrast, diets supplemented with resistant starch tended to enrich the Lactobacillus population in mouse caecum, but much less at high doses of resistant starch ${ }^{52}$. Incidentally, hydrogenase genes, which encode enzymes for the reversible oxidation of $\mathrm{H}_{2}$, were recently shown to be completely absent in Bacilli and bifidobacteria ${ }^{51}$. Considering the lack of a correlation between $\mathrm{H}_{2}$ production and Lactobacillus in our study, new questions emerge about the ability of Lactobacillus to thrive in $\mathrm{H}_{2}$-poor environments.

The increase in isovaleric acid, a product of branched-chain amino acid catabolism ${ }^{65}$, in HDD-fed mice, suggests a shift of microbiota towards protein fermentation. Bacteria from the genera Enterorhabdus ${ }^{66}$ and Parvibacter ${ }^{67}$, both significantly induced by HDD-feeding, have the ability to ferment amino acids. Additionally Olsenella, only present in two samples in the HDD group, is documented to grow on tyrosine and produce $p$-cresol ${ }^{68}$, supporting our hypothesis for a shift to protein fermentation. This might have important implications for the host, since products of protein fermentation such as phenols, ammonia, certain amines, and $\mathrm{H}_{2} \mathrm{~S}$, are considered to play important roles in the initiation or progression of bowel diseases, inflammation, DNA damage, and cancer ${ }^{69}$.

Altogether, our results emphasize $\mathrm{H}_{2}$ as a key factor within the intestinal microenvironment and the usefulness of knowing its production dynamics to understand the interplay between host, diet, and the intestinal microbiota. At the same time, we are aware that our approach to study such interactions may have conceivable limitations. It has been argued that changes in gas evolution (and other indirect markers of fermentation) cannot accurately indicate changes in fermentation ${ }^{70}$, and even "real-time", carefully controlled measurements have failed to show quantitative changes in $\mathrm{H}_{2}$ and $\mathrm{CH}_{4}$ production proportionally linked to consumption of fermentable carbohydrates ${ }^{15,41}$. We completely agree with these authors that the measured outcomes, $\mathrm{H}_{2}$ and $\mathrm{CH}_{4}$, not only reflect the type of carbohydrate consumed, but are the end result of a very complex fermentation stoichiometry that depends on the host's capacity to digest and absorb nutrients, the dominance and metabolic activity of microbial species, and their interactions. However, the conclusion that fermentation gases are extremely limited parameters to study carbohydrate fermentation is largely based on human data, where eating pattern, environment, genetic variation, and the gut microbe interact and ultimately determine an individual's response to the diet ${ }^{71,72}$. When these and other factors can be better controlled, as it is the case with animal models, the analysis of carbohydrate fermentation through $\mathrm{H}_{2}$ and $\mathrm{CH}_{4}$ quantification has much to offer. The fact that in vitro models to measure $\mathrm{H}_{2}$ and $\mathrm{CH}_{4}$ evolution are still developing and proposed as a tool to unravel the mechanisms behind the association between microbiota and host health ${ }^{73}$ is encouraging. 


\section{CHAPTER 2}

Overall, the applications of gas analysis within an indirect calorimetry system go beyond the arena of carbohydrate quality and nutritional studies, and may be used as a diagnosis tool in clinical practice ${ }^{19,74,75}$. It opens up new avenues not only in preclinical research in rodents, but also has potential in human-diet-microbiota interaction studies if such sensor technology is incorporated into indirect calorimetry chambers or ventilated hood systems.

\section{Conclusions}

Using our customized indirect calorimetry system we were able to continuously quantify $\mathrm{H}_{2}$ production in mice as a reflection of the starch digestibility of the diet. $\mathrm{H}_{2}$ monitoring also allowed us to catch the earliest stages in the adaptation to carbohydrates of different digestibility, revealing a nuanced process with high inter-individual variation. Importantly, in vivo $\mathrm{H}_{2}$ production was significantly correlated with specific microbial taxa, including Bacteroides and Parasutterella. The implemented $\mathrm{H}_{2}$ and $\mathrm{CH}_{4}$ sensor-technology described here opens yet unmet avenues to study the effects of nutrition on microbiota in real time, not only in rodents, but potentially also in humans.

\section{Author Contributions}

J.K. and E.M.v.S. conceived, designed, and supervised the project. J.M.S.F.-C., H.J.M.S., and L.M.S.B. conducted animal experiments. H.J.M.S. and E.M.v.S. integrated the $\mathrm{H}_{2}$ and $\mathrm{CH}_{4}$ sensors into the indirect calorimetry system. L.M.S.B., A.O., and H.S. provided input for experimental design and interpretation of results. N.B. and V.G.-C. analysed and interpreted in vitro digestion data. P.K. and H.S. carried out all microbiome analyses and interpreted the data. J.M.S.F.-C., P.K., E.M.v.S., J.K., and H.S. interpreted data and prepared the manuscript. All authors critically revised and approved the manuscript.

\section{Acknowledgements}

We acknowledge the help of the personnel at the CARUS animal facility. We also thank Inge van der Stelt and students Laura Dewitte and Lini Sholihah for their help in collecting data. 


\section{References}

Englyst, K. N., Liu, S. \& Englyst, H. N. Nutritional characterization and measurement of dietary carbohydrates. Eur. J. Clin. Nutr. 61 Suppl 1, S19-39 (2007).

Elia, M. \& Cummings, J. H. Physiological aspects of energy metabolism and gastrointestinal effects of carbohydrates. Eur. J. Clin. Nutr. 61 Suppl 1, S40-74 (2007).

den Besten, G. et al. The role of short-chain fatty acids in the interplay between diet, gut microbiota, and host energy metabolism. J. Lipid. Res. 54, 2325-2340 (2013).

Silk, D. B., Webb, J. P., Lane, A. E., Clark, M. L. \& Dawson, A. M. Functional differentiation of human jejunum and ileum: a comparison of the handling of glucose, peptides, and amino acids. Gut 15, $444-$ 449 (1974).

Flint, H. J., Scott, K. P., Louis, P. \& Duncan, S. H. The role of the gut microbiota in nutrition and health. Nat. Rev. Gastroenterol. Hepatol. 9, 577-589 (2012).

Fischbach, M. A. \& Sonnenburg, J. L. Eating for two: how metabolism establishes interspecies interactions in the gut. Cell Host Microbe 10, 336-347 (2011).

Hooper, L. V. et al. Molecular analysis of commensal host-microbial relationships in the intestine. Science 291, 881-884 (2001).

Cox, L. M. et al. Altering the intestinal microbiota during a critical developmental window has lasting metabolic consequences. Cell 158, 705-721 (2014).

van Nood, E. et al. Duodenal infusion of donor feces for recurrent Clostridium difficile. N. Engl. J. Med. 368, 407-415 (2013).

Martinez-Fernandez, G., Denman, S. E., Cheung, J. \& McSweeney, C. S. Phloroglucinol degradation in the rumen promotes the capture of excess hydrogen generated from methanogenesis inhibition. Front. Microbiol. 8, 1871 (2017).

Olijhoek, D. W. et al. Effect of dietary nitrate level on enteric methane production, hydrogen emission, rumen fermentation, and nutrient digestibility in dairy cows. J. Dairy Sci. 99, 6191-6205 (2016).

Rooke, J. A. et al. Hydrogen and methane emissions from beef cattle and their rumen microbial community vary with diet, time after feeding and genotype. Br. J. Nutr. 112, 398-407 (2014).

Sollinger, A. et al. Holistic assessment of rumen microbiome dynamics through quantitative metatranscriptomics reveals multifunctional redundancy during key steps of anaerobic feed degradation. mSystems 3, e00038-18 (2018).

van Lingen, H. J. et al. Diurnal dynamics of gaseous and dissolved metabolites and microbiota composition in the bovine rumen. Front. Microbiol. 8, 425 (2017).

Christl, S. U., Murgatroyd, P. R., Gibson, G. R. \& Cummings, J. H. Production, metabolism, and excretion of hydrogen in the large intestine. Gastroenterology 102, 1269-1277 (1992).

Symonds, E. L., Kritas, S., Omari, T. I. \& Butler, R. N. A combined ${ }^{13} \mathrm{CO}_{2} / \mathrm{H}_{2}$ breath test can be used to assess starch digestion and fermentation in humans. J. Nutr. 134, 1193-1196 (2004).

Ong, D. K. et al. Manipulation of dietary short chain carbohydrates alters the pattern of gas production and genesis of symptoms in irritable bowel syndrome. J. Gastroenterol. Hepatol. 25, 13661373 (2010).

Valeur, J., Puaschitz, N. G., Midtvedt, T. \& Berstad, A. Oatmeal porridge: impact on microfloraassociated characteristics in healthy subjects. Br. J. Nutr. 115, 62-67 (2016).

Carbonero, F., Benefiel, A. C. \& Gaskins, H. R. Contributions of the microbial hydrogen economy to colonic homeostasis. Nat. Reu. Gastroenterol. Hepatol. 9, 504-518 (2012).

Isken, F., Klaus, S., Osterhoff, M., Pfeiffer, A. F. \& Weickert, M. O. Effects of long-term soluble vs. insoluble dietary fiber intake on high-fat diet-induced obesity in C57BL/6] mice. J. Nutr. Biochem. 21, 278-284 (2010). 


\section{CHAPTER 2}

Hartmann, L., Taras, D., Kamlage, B. \& Blaut, M. A new technique to determine hydrogen excreted by gnotobiotic rats. Lab. Anim. 34, 162-170 (2000).

Ostrander, C. R., Stevenson, D. K., Neu, J., Kerner, J. A. \& Moses, S. W. A sensitive analytical apparatus for measuring hydrogen production rates. I. Application to studies in small animals. Evidence of the effects of an 回-glucosidehydrolase inhibitor in the rat. Anal. Biochem. 119, 378-386 (1982).

Dufourlescoat, C., Lecoz, Y., Andrieux, C. \& Szylit, O. Effects of nature, size and level of incorporation of dietary fibers on colonic functions in germ-free rats and in heteroxenic rats inoculated with a human flora. Food. Hydrocoll. 9, 9-15 (1995).

Rodkey, F. L., Collison, H. A. \& O’Neal, J. D. Carbon monoxide and methane production in rats, guinea pigs, and germ-free rats. J. Appl. Physiol. 33, 256-260 (1972).

Walter, D. J., Eastwood, M. A., Brydon, W. G. \& Elton, R. A. An experimental design to study colonic fibre fermentation in the rat: the duration of feeding. Br. J. Nutr. 55, 465-479 (1986).

Tuboly, E. et al. Determination of endogenous methane formation by photoacoustic spectroscopy. J. Breath Res. 7, 046004 (2013).

Hoevenaars, F. P. et al. Effects of dietary history on energy metabolism and physiological parameters in C57BL/6] mice. Exp. Physiol. 98, 1053-1062 (2013).

Duivenvoorde, L. P. et al. Oxygen restriction as challenge test reveals early high-fat-diet-induced changes in glucose and lipid metabolism. Pflugers Arch. 467, 1179-1193 (2015).

Reeves, P. G., Nielsen, F. H. \& Fahey, G. C., Jr. AIN-93 purified diets for laboratory rodents: final report of the American Institute of Nutrition ad hoc writing committee on the reformulation of the AIN-76A rodent diet. J. Nutr. 123, 1939-1951 (1993).

Isken, F. et al. Impairment of fat oxidation under high-vs. low-glycemic index diet occurs before the development of an obese phenotype. Am. J. Physiol. Endocrinol. Metab. 298, E287-295 (2010).

Garcia-Campayo, V., Han, S., Vercauteren, R. \& Franck, A. Digestion of Food Ingredients and Food Using an In Vitro Model Integrating Intestinal Mucosal Enzymes. Food and Nutrition Sciences 09, 711 734 (2018).

Versantvoort, C. H., Oomen, A. G., Van de Kamp, E., Rompelberg, C. J. \& Sips, A. J. Applicability of an in vitro digestion model in assessing the bioaccessibility of mycotoxins from food. Food Chem. Toxicol. 43, 31-40 (2005).

Morin, L. G. \& Prox, J. Single glucose oxidase-peroxidase reagent for two-minute determination of serum glucose. Clin. Chem. 19, 959-962 (1973).

Ladirat, S. E. et al. Impact of galacto-oligosaccharides on the gut microbiota composition and metabolic activity upon antibiotic treatment during in vitro fermentation. FEMS Microbiol. Ecol. 87, 41-51 (2014).

Ramiro-Garcia, J. et al. NG-Tax, a highly accurate and validated pipeline for analysis of 16S rRNA amplicons from complex biomes. F100ORes. 5, 1791 (2016).

Lahti, L. \& Shetty, S. Tools for microbiome analysis in R. Version 0.99.90., http://microbiome.github.io/ microbiome/ (2017).

Caporaso, J. G. et al. QIIME allows analysis of high-throughput community sequencing data. Nat. Methods 7, 335-336 (2010).

Duivenvoorde, L. P., van Schothorst, E. M., Swarts, H. J. \& Keijer, J. Assessment of metabolic flexibility of old and adult mice using three noninvasive, indirect calorimetry-based treatments. J. Gerontol. A Biol. Sci. Med. Sci. 70, 282-293 (2015). 
Poppitt, S. D. et al. Circadian patterns of total 24-h hydrogen and methane excretion in humans ingesting nonstarch polysaccharide (NSP) diets and the implications for indirect calorimetric and $D_{2}$ ${ }^{18} \mathrm{O}$ methodologies. Eur. J. Clin. Nutr. 50, 524-534 (1996).

Tulley, R. T. et al. Comparative methodologies for measuring metabolizable energy of various types of resistant high amylose corn starch. J. Agric. Food Chem. 57, 8474-8479 (2009).

Nishimura, N., Tanabe, H. \& Yamamoto, T. Isomaltodextrin, a highly branched alpha-glucan, increases rat colonic $\mathrm{H}_{2}$ production as well as indigestible dextrin. Biosci. Biotechnol. Biochem. 80, 554-563 (2016).

Tonouchi, H. et al. Studies on absorption and metabolism of palatinose (isomaltulose) in rats. Br. J. Nutr. 105, 10-14 (2011).

Kalantar-Zadeh, K. et al. A human pilot trial of ingestible electronic capsules capable of sensing different gases in the gut. Nat. Electron. 1, 79-87 (2018).

Levrat, M. A., Remesy, C. \& Demigne, C. Very acidic fermentations in the rat cecum during adaptation to a diet rich in amylase-resistant starch (crude potato starch). J. Nutr. Biochem. 2, 31-36 (1991).

Key, F. B. \& Mathers, J. C. Digestive adaptations of rats given white bread and cooked haricot beans (Phaseolus vulgaris): large-bowel fermentation and digestion of complex carbohydrates. Br. J. Nutr. 74, 393-406 (1995).

Walker, A. W. et al. Dominant and diet-responsive groups of bacteria within the human colonic microbiota. ISME J. 5, 220-230 (2011).

Kohl, K. D., Amaya, J., Passement, C. A., Dearing, M. D. \& McCue, M. D. Unique and shared responses of the gut microbiota to prolonged fasting: a comparative study across five classes of vertebrate hosts. FEMS Microbiol. Ecol. 90, 883-894 (2014).

Crawford, P. A. et al. Regulation of myocardial ketone body metabolism by the gut microbiota during nutrient deprivation. Proc. Natl. Acad. Sci. USA 106, 11276-11281 (2009).

Wolf, P. G., Biswas, A., Morales, S. E., Greening, C. \& Gaskins, H. R. H metabolism is widespread and diverse among human colonic microbes. Gut Microbes 7, 235-245 (2016).

Tachon, S., Zhou, J., Keenan, M., Martin, R. \& Marco, M. L. The intestinal microbiota in aged mice is modulated by dietary resistant starch and correlated with improvements in host responses. FEMS Microbiol. Ecol. 83, 299-309 (2013).

Barouei, J. et al. Microbiota, metabolome, and immune alterations in obese mice fed a high-fat diet containing type 2 resistant starch. Mol. Nutr. Food Res. 61, 1700184 (2017).

Muyzer, G. \& Stams, A. J. The ecology and biotechnology of sulphate-reducing bacteria. Nat. Reu. Microbiol. 6, 441-454 (2008).

Turnbaugh, P. J. et al. An obesity-associated gut microbiome with increased capacity for energy harvest. Nature 444, 1027-1031 (2006).

Laverdure, R., Mezouari, A., Carson, M. A., Basiliko, N. \& Gagnon, J. A role for methanogens and methane in the regulation of GLP-1. Endocrinol. Diabetes Metab. 1 (2018).

57 Attene-Ramos, M. S., Wagner, E. D., Plewa, M. J. \& Gaskins, H. R. Evidence that hydrogen sulfide is a genotoxic agent. Mol. Cancer Res. 4, 9-14 (2006).

Roediger, W. E., Duncan, A., Kapaniris, O. \& Millard, S. Sulphide impairment of substrate oxidation in rat colonocytes: a biochemical basis for ulcerative colitis? Clin. Sci. 85, 623-627 (1993).

Attene-Ramos, M. S., Wagner, E. D., Gaskins, H. R. \& Plewa, M. J. Hydrogen sulfide induces direct radical-associated DNA damage. Mol. Cancer. Res. 5, 455-459 (2007).

Soriano, R. N. et al. Endogenous peripheral hydrogen sulfide is propyretic: its permissive role in brown adipose tissue thermogenesis in rats. Exp. Physiol. 103, 397-407 (2018).

Segata, N. et al. Composition of the adult digestive tract bacterial microbiome based on seven mouth surfaces, tonsils, throat and stool samples. Genome Biol. 13, R42 (2012). 


\section{CHAPTER 2}

62 Goker, M. et al. Complete genome sequence of Odoribacter splanchnicus type strain $\left(1651 / 6^{\top}\right)$. Stand. Genomic. Sci. 4, 200-209 (2011).

63 Bomar, L., Maltz, M., Colston, S. \& Graf, J. Directed culturing of microorganisms using metatranscriptomics. MBio 2, e00012-00011 (2011).

64 Tsai, H. H., Hart, C. A. \& Rhodes, J. M. Production of mucin degrading sulphatase and glycosidases by Bacteroides thetaiotaomicron. Lett. Appl. Microbiol. 13, 97-101 (1991).

65 Dai, Z. L., Wu, G. Y. \& Zhu, W. Y. Amino acid metabolism in intestinal bacteria: links between gut ecology and host health. Front. Biosci. (Landmark Ed.) 16, 1768-1786 (2011).

66 Clavel, T. et al. Isolation of bacteria from the ileal mucosa of TNFdeltaARE mice and description of Enterorhabdus mucosicola gen. nov., sp. nov. Int. J. Syst. Evol. Microbiol. 59, 1805-1812 (2009).

67 Clavel, T., Charrier, C., Wenning, M. \& Haller, D. Parvibacter caecicola gen. nov., sp. nov., a bacterium of the family Coriobacteriaceae isolated from the caecum of a mouse. Int. J. Syst. Evol. Microbiol. 63, 2642-2648 (2013).

68 Li, X., Jensen, R. L., Hojberg, O., Canibe, N. \& Jensen, B. B. Olsenella scatoligenes sp. nov., a 3-methylindole- (skatole) and 4-methylphenol- ( $p$-cresol) producing bacterium isolated from pig faeces. Int. J. Syst. Evol. Microbiol. 65, 1227-1233 (2015).

69 Windey, K., De Preter, V. \& Verbeke, K. Relevance of protein fermentation to gut health. Mol. Nutr. Food. Res. 56, 184-196 (2012).

Topping, D. L. \& Clifton, P. M. Short-chain fatty acids and human colonic function: roles of resistant starch and nonstarch polysaccharides. Physiol. Reu. 81, 1031-1064 (2001).

Zoetendal, E. G., Akkermans, A. D. L., Akkermans-van Vliet, W. M., de Visser, J. A. G. M. \& de Vos, W. $M$. The host genotype affects the bacterial community in the human gastronintestinal tract. Microb. Ecol. Health Dis. 13, 129-134 (2009).

Zeevi, D. et al. Personalized nutrition by prediction of glycemic responses. Cell 163, 1079-1094 (2015).

Carter, E. A. \& Barr, R. G. Preliminary studies demonstrating acetoclastic methanogenesis in a rat colonic ring model. J. Nutr. Metab. 2013, 540967 (2013).

Pimentel, M. Breath testing for small intestinal bacterial overgrowth: should we bother? Am. J. Gastroenterol. 111, 307-308 (2016).

(2013). 

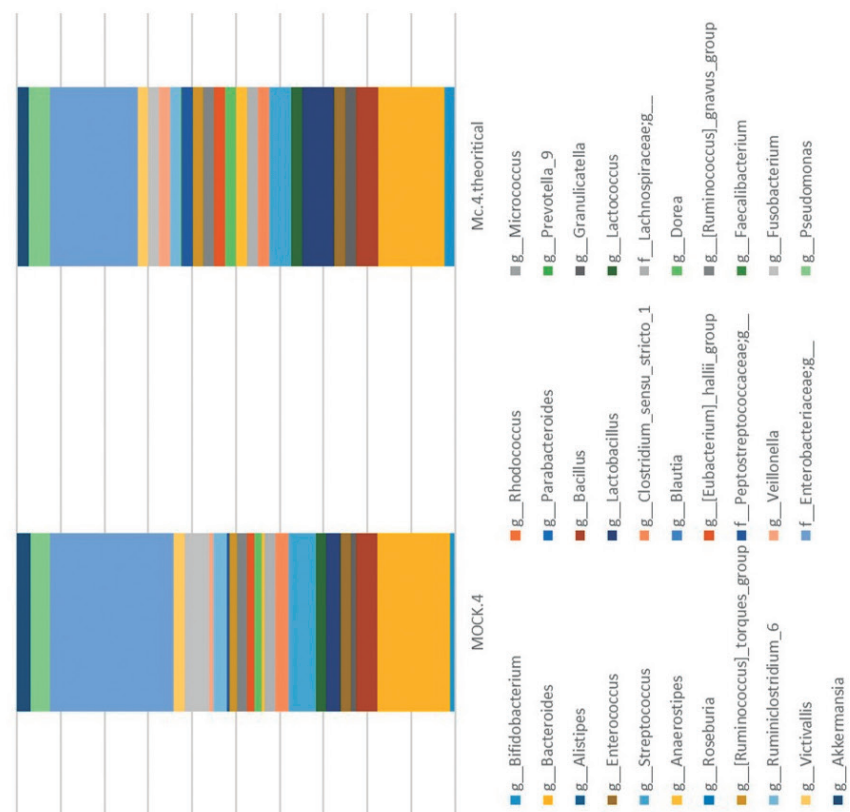

으
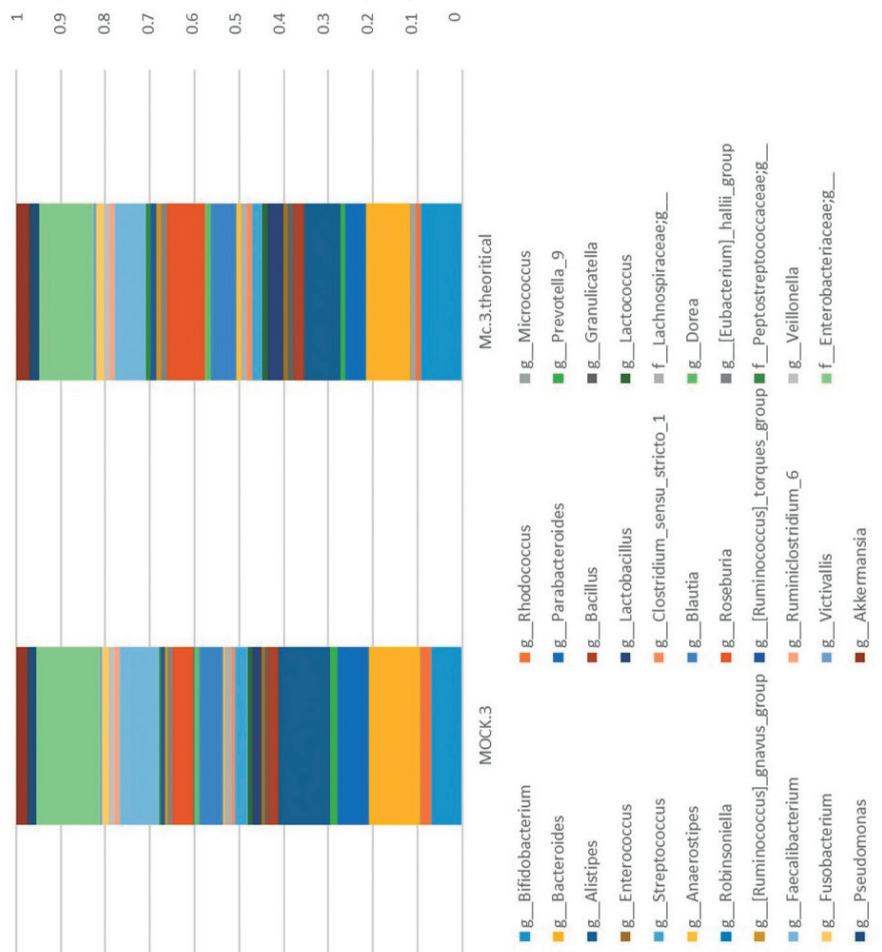

중

(5)

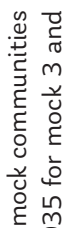

मे

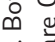

o

वं

눙

iे

둥

है

ક

0.

. $\frac{\text { है }}{\mathrm{v}}$

可

호.

은 온

ᄃ

要

岁

ڤँ

पै

兵

幽

용

灵

है 응

美

둥

范

के है

ऐ व

․

兵

은

क :

के के

ह

त्व

更

可 궁

$\infty \frac{0}{\pi}$

के है है

० 원

苟

एँ 


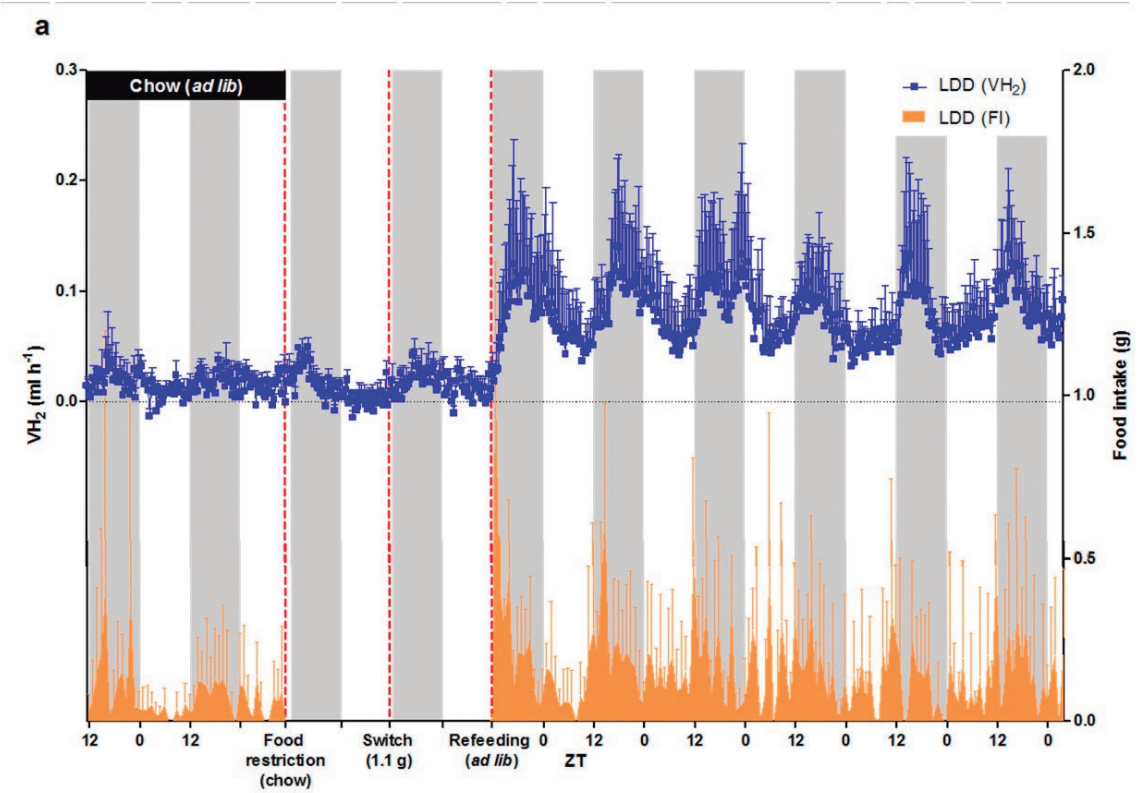

b

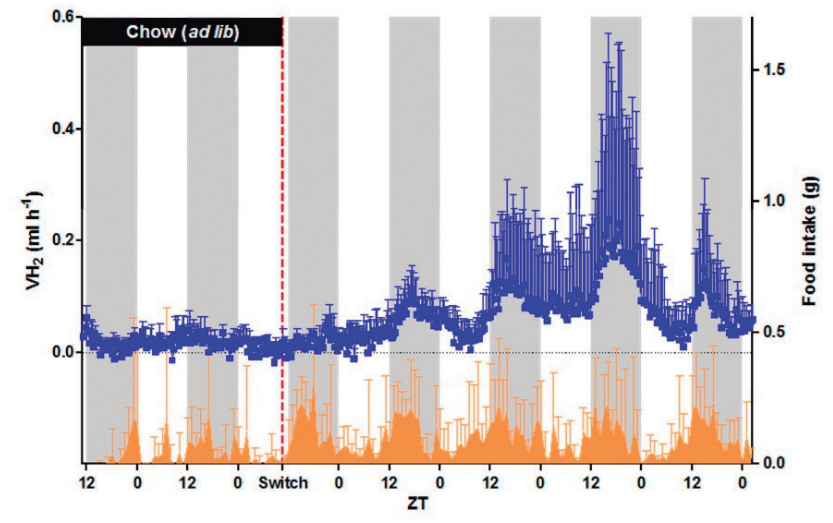

Figure S2. Hydrogen $\left(\mathrm{H}_{2}\right)$ production closely follows food intake (FI) patterns. (a) Mice within indirect calorimetry were fed standard chow, followed by chow restriction leading to a fasting state (left dotted line), which was subsequently followed by feeding $1.1 \mathrm{~g}$ of LDD (blue; $n=4$ ) prior to the dark phase as a single meal test (2nd dotted line). As a result, mice were fasted the next day, and received prior to next dark phase ad libitum access to LDD (3rd dotted line) for an additional $5.5 \mathrm{~d}$. (b) Chow-fed mice $(n=6)$ were switched to LDD without prior food restriction and measurements continued for another $4.5 \mathrm{~d}$. All mice received no other diet than chow during their whole lifetime prior to these experiments until the dietary switch (black bar), but colour usage reflects exposure to new diets. Volume of $\mathrm{H}_{2}$ produced $\left(\mathrm{VH}_{2}\right.$; blue squares, left $\mathrm{y}$-axis, $\mathrm{ml}$ h-1) is plotted together with associated hourly food intake episodes (orange area, right y-axis, g), except when food was placed directly inside the cage and not in the food baskets (food restriction, panel a). White and grey areas represent light and dark phases, respectively. Data is presented as mean \pm s.d. For clarity, only upper error bars are shown. ZT, Zeitgeber time. 
a

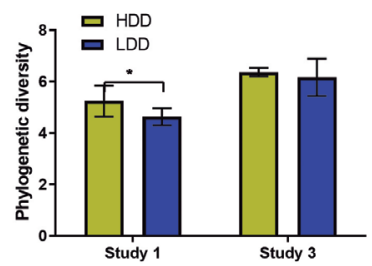

b

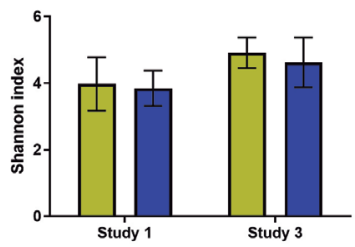

C

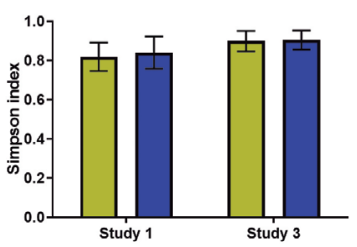

Figure S3. Metrics of $\alpha$-diversity in faecal microbiota of HDD- or LDD-fed mice. Phylogenetic diversity (a), and Shannon (b) and Simpson (c) indexes of mice fed HDD or LDD for 3 weeks ( $n=12$ per diet, Study 1$)$ or $4.5 d(n=5$ per diet, Study 3). Statistical comparisons were made using unpaired two-tailed Student's $t$-test; ${ }^{*} P \leq 0.05$. Data shown as mean \pm s.d.

Figure S4 (next page). Exposure to starches of different digestibility induces distinct microbial taxa. All bacterial genera that were significantly increased by LDD (a) or HDD (c) after 3 weeks of exposure to the diets ( $n=12$ per diet, Study 1) are shown. All genera that were significantly increased by LDD (b) or HDD (d) after $4.5 \mathrm{~d}$ of exposure to the diets ( $n=5$ per diet, Study 3 ) are also shown for comparison. Side-by-side boxes represent the $\log _{10}$ transformed LDA scores of bacterial taxa enriched in HDD- or LDD-fed mice in Study 1 (e) and Study 3 (f) analysed by LEfSe. For uncultured genera, family name is indicated $\left(f \_\right)$. Comparisons were done by non-parametric $t$-test followed by 999 permutations and $P$ values were adjusted by False Discovery Rate (FDR) correction; ${ }^{*} P<0.1,{ }^{*} P \leq$ $0.05,{ }^{* *} P \leq 0.01$. Data is presented as mean \pm s.d. 

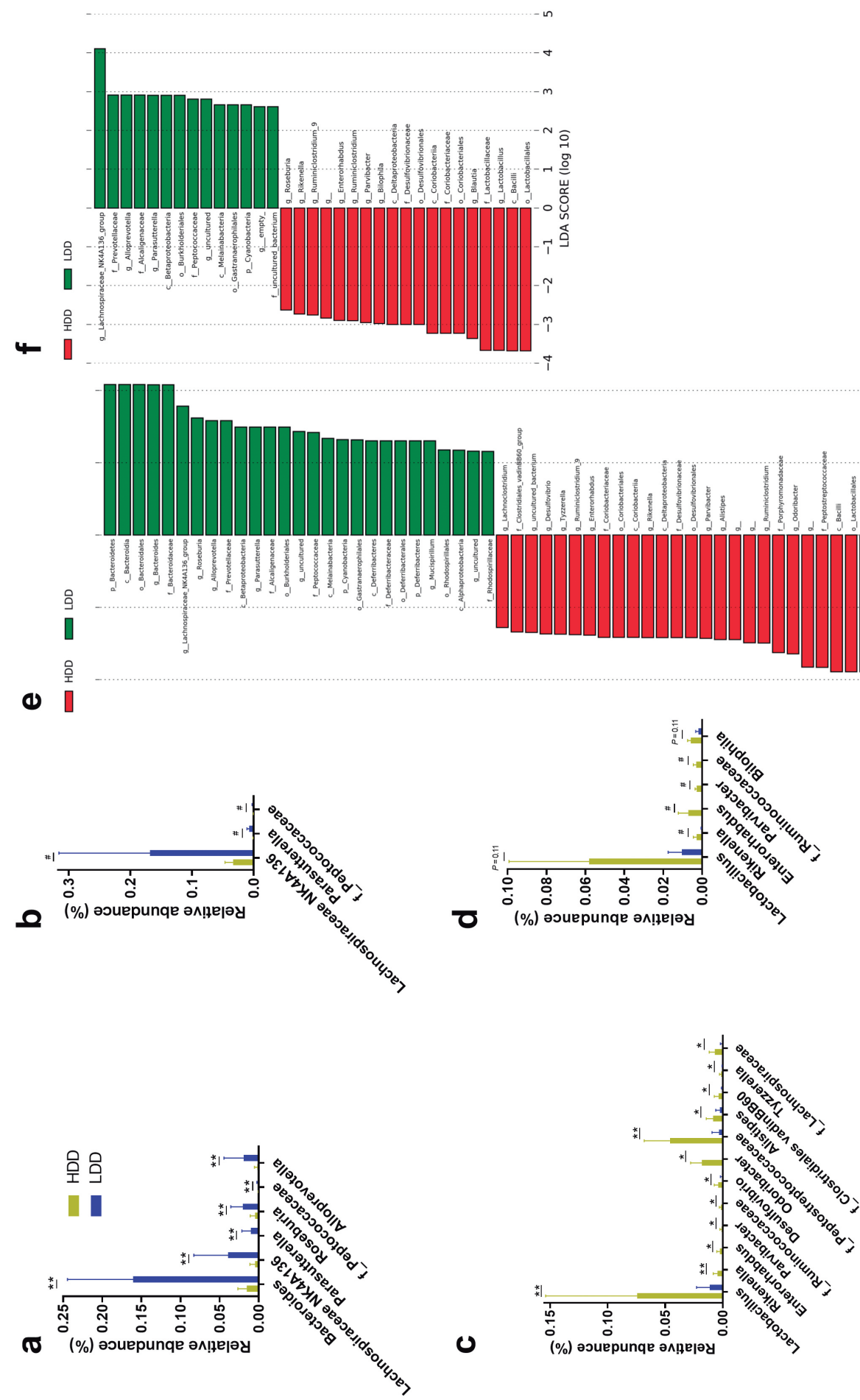

Non-Invasive Continuous Real-Time In Vivo Analysis of Microbial Hydrogen Production Shows Adaptation to Fermentable Carbohydrates in Mice 


\section{a}

HDD 3wks

HDD $4.5 d$

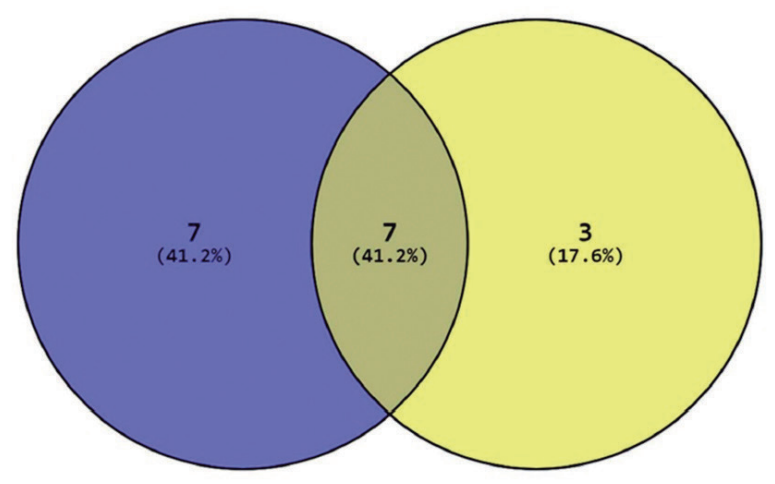

b

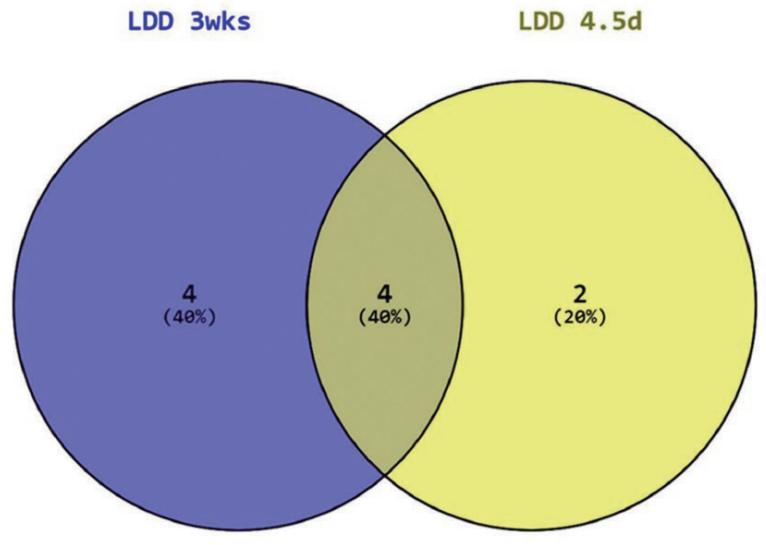

Figure S5. Venn diagrams showing the unique and shared genera which are significantly enriched as determined by the LefSe analysis between mice (a) exposed to HDD and (b) to LDD.

Figure S6 (next page). Specific bacterial genera correlating with in vivo $\mathrm{H}_{2}$ production. (a) Positive and (b) negative Spearman's rank correlations of specific faecal bacterial genera and $\mathrm{H}_{2}$ production of mice exposed to HDD or LDD for 3 weeks after weaning ( $n=12$ per diet, Study 1$)$; FDR threshold set to $P<0.1$. 

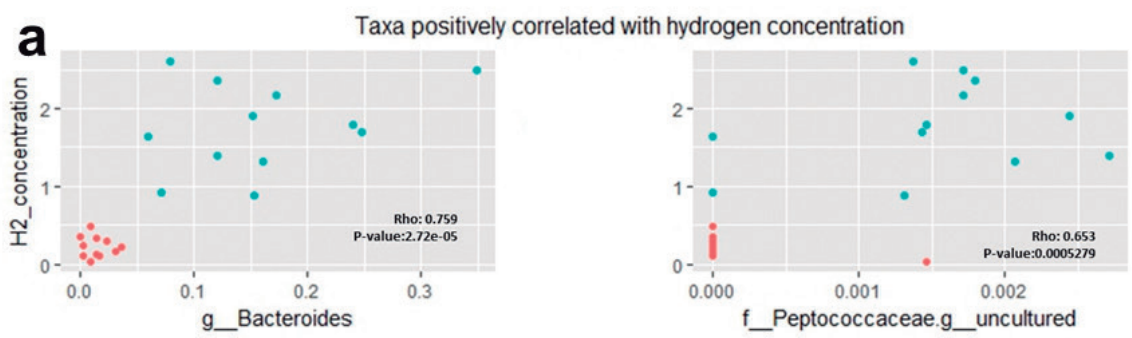

Diet

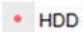

- LDD
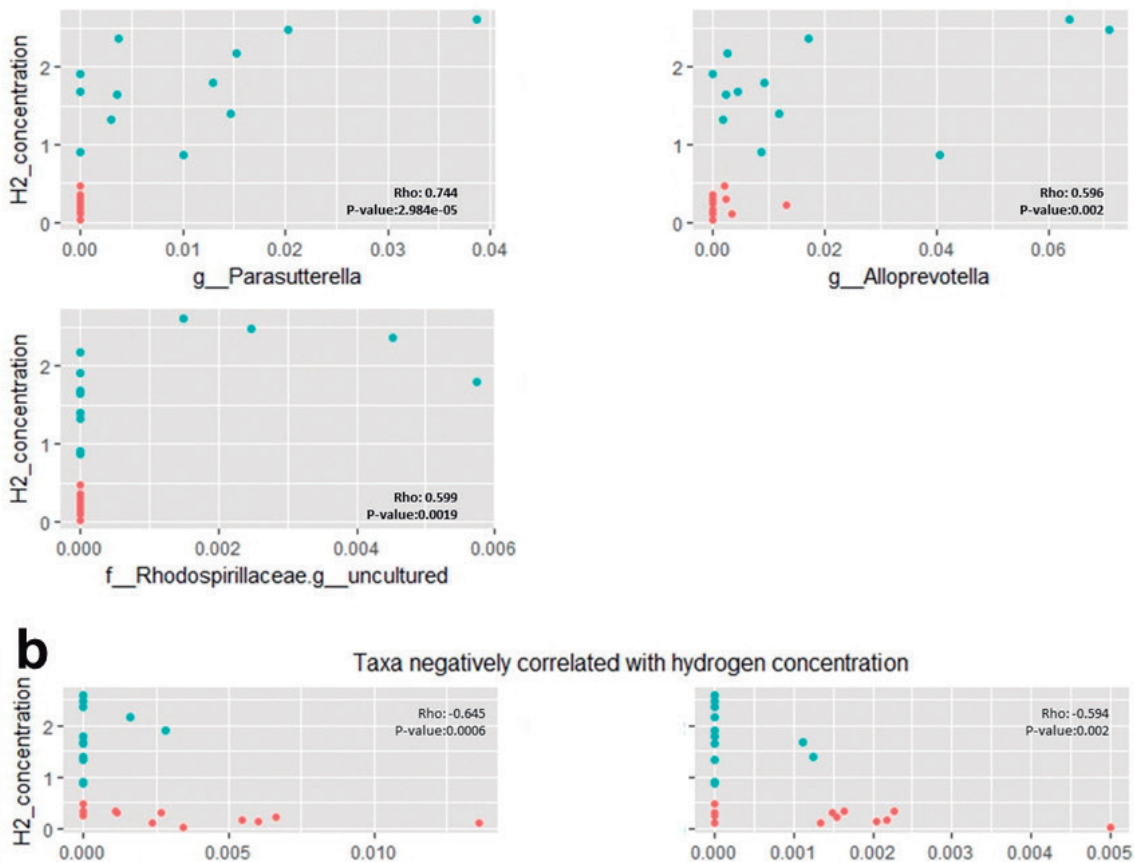

Clostridiales_vadinBB60_group.g_uncultured_bacterium
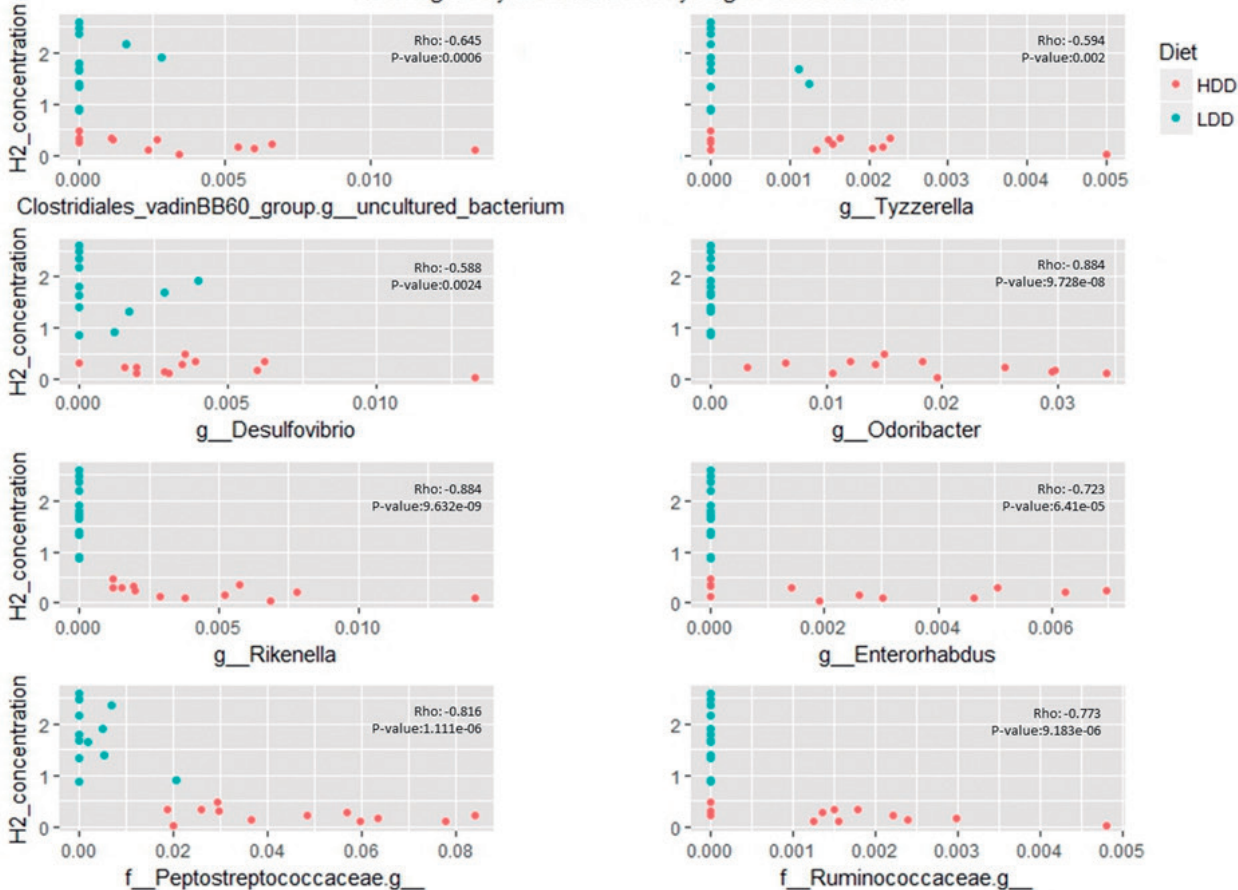

Non-Invasive Continuous Real-Time In Vivo Analysis of Microbial Hydrogen Production Shows Adaptation to Fermentable Carbohydrates in Mice 


\section{Chapter}

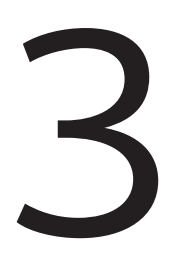

\section{Extended Indirect Calorimetry with Isotopic $\mathrm{CO}_{2}$ Sensors for Prolonged and Continuous Quantification of Exogenous us. Total Substrate Oxidation in Mice}

José M. S. Fernández-Calleja

Lianne M. S. Bouwman

Hans J. M. Swarts

Annemarie Oosting

Jaap Keijer

Evert M. van Schothorst 



\begin{abstract}
Indirect calorimetry ( $\mathrm{InC}$ ) estimates whole-body energy expenditure and total substrate oxidation based on $\mathrm{O}_{2}$ consumption and $\mathrm{CO}_{2}$ production, but does not allow to quantify oxidation of exogenous substrates with time. To achieve this, we here incorporated ${ }^{13} \mathrm{CO}_{2}$ and ${ }^{12} \mathrm{CO}_{2}$ gas sensors into a commercial InCa system and aimed to demonstrate their performance and added value. As performance indicator, we showed that discriminative oscillations in ${ }^{13} \mathrm{CO}_{2}$ enrichment associated with food intake in mice fed diets containing naturally low (wheat) us high (maize) ${ }^{13} \mathrm{C}$ enrichment were measurable. To demonstrate the physiological added value, we quantified exogenous us total carbohydrate and fat oxidation continuously, in real time in mice varying in fat mass. Diet-induced obese mice were fed a single liquid mixed meal containing ${ }^{13} \mathrm{C}$-isotopic tracers of glucose or palmitate. Kinetics of immediate glucose and palmitate oxidation differed. Over $13 \mathrm{~h}, \sim 70 \%$ glucose and $\sim 48 \%$ palmitate ingested were oxidised. Exogenous palmitate oxidation depended on body fat mass, while this was not the case for exogenous glucose oxidation. We conclude that extending an InCa system with ${ }^{13} \mathrm{CO}_{2}$ and ${ }^{12} \mathrm{CO}_{2}$ sensors provides an accessible and powerful technique for real-time continuous quantification of exogenous and whole-body substrate oxidation in mouse models of human metabolic physiology.
\end{abstract}

Keywords: Isotope labelling, C57BL mice, metabolic flexibility, obesity, metabolism, substrate oxidation. 


\section{Introduction}

Indirect calorimetry ( $\operatorname{InCa}$ ) has been essential to understand human wholebody energy metabolism and fuel selection for more than a century ${ }^{1}$. It allows for the estimation of energy expenditure (EE) and of substrate utilisation by calculation of the respiratory exchange ratio (RER) from $\mathrm{O}_{2}$ consumption and $\mathrm{CO}_{2}$ production measurements. In the mouse, a commonly used model animal for human physiology, indirect calorimetry is the preferred technique to measure energy expenditure continuously ${ }^{2}$. However, estimations of RER represent only the whole-body net balance of substrates oxidised and do not distinguish between endogenous and exogenous (dietary) metabolic substrates. This knowledge can be obtained with the use of metabolic tracers, which are compounds that behave identically to the compound of interest, but can be analytically distinguished from them, for instance by mass difference.

The stable natural isotope ${ }^{13} \mathrm{C}$ has been widely used in metabolic studies for the past five decades ${ }^{3}$. Next to ${ }^{12} \mathrm{C},{ }^{13} \mathrm{C}$ is the second most abundant isotope of elemental carbon, representing about $1 \%$ of total terrestrial carbon ${ }^{4}$. Based on their distinct $\mathrm{CO}_{2}$ fixation mechanisms, plants classified as C4 (e.g. maize or corn, and sugar cane) have a naturally higher ${ }^{13} \mathrm{C}$ abundance compared to $\mathrm{C} 3$ plants (e.g. wheat and sugar beet), and ingredients derived from $\mathrm{C} 4$ plants are often used as natural metabolic tracers ${ }^{5}$.

Upon oxidation in the body, ${ }^{13} \mathrm{C}$ atoms in metabolic substrates are excreted as ${ }^{13} \mathrm{CO}_{2}$ which can be measured in breath samples and is usually given as atom $\%{ }^{13} \mathrm{CO}_{2}$ enrichment ${ }^{3}$. While this provides a qualitative measurement of exogenous substrate oxidation, calculating actual substrate oxidation rates gives better insights into metabolic physiology and allows direct comparison between exogenous and endogenous substrates. Such quantitative measurements require knowledge of $\mathrm{CO}_{2}$ production rates, and this has been regularly determined in human studies by $\mathrm{InCa}^{3}$. Only recently, livestock animal nutritionists and comparative biologists have also started to combine ${ }^{13} \mathrm{CO}_{2}$ enrichment analysis with $\mathrm{InCa}$ to calculate substrate oxidation rates ${ }^{5-8}$. Although informative, metabolic tracer research in animal models, like the mouse, rarely includes measurements of $\mathrm{CO}_{2}$ production rates and the number of breath samples that can be collected and analysed for ${ }^{13} \mathrm{CO}_{2}$ enrichment is limited ${ }^{9-13}$. Continuous quantification of total $\mathrm{CO}_{2}$ production together with ${ }^{13} \mathrm{CO}_{2}$ enrichment would allow precise measurement of responses to metabolic tracers in models of human physiology. To our knowledge, there have been only two attempts to obtain quantitative measurements of substrate oxidation in mice using ${ }^{13} \mathrm{CO}_{2}$ enrichment data in tandem with InCa over prolonged study times, and they involved expensive equipment ${ }^{14}$ or labour-intensive sampling ${ }^{15}$.

We have recently shown that a commercial InCa system can be successfully extended to incorporate analysis of other gases ${ }^{16}$, in this case measurement of the gut microbiota fermentation gases. The inclusion of hydrogen $\left(\mathrm{H}_{2}\right)$ and methane $\left(\mathrm{CH}_{4}\right)$ sensors resulted in a system that offers a tool for more detailed phenotyping than conventional InCa for nutritional interventions in mice. Here, we have also incorporated ${ }^{13} \mathrm{CO}_{2}$ and ${ }^{12} \mathrm{CO}_{2}$ sensors into the same commercial InCa system and demonstrate their usefulness technically and 
physiologically. The system was able to characterise changes in natural ${ }^{13} \mathrm{CO}_{2}$ enrichment based on $24 \mathrm{~h}$ feeding cycles and the consumption of diets with distinct ${ }^{13} \mathrm{C}$ signatures. Moreover, real-time ${ }^{13} \mathrm{CO}_{2}$ enrichment measurements linked to conventional InCa added value to current established metabolic phenotyping methodologies such as refeeding challenge tests, by not only quantifying exogenous and endogenous oxidation rates, but also quantifying the oxidative disposal of glucose and fat ingested with a meal in the context of diet-induced obesity.

\section{Materials and Methods}

Integration of ${ }^{13} \mathrm{CO}_{2}$ and ${ }^{12} \mathrm{CO}_{2}$ sensors into the indirect calorimetry (InCa) system

An Infrared Analyser Module URAS26 for separate analysis of ${ }^{13} \mathrm{CO}_{2}$ and ${ }^{12} \mathrm{CO}_{2}$ by nondispersive infrared absorption (ABB Automation, Frankfurt am Main, Germany) was incorporated into a 12-cage PhenoMaster InCa system (TSE Systems, Bad Homburg, Germany) in a closed circuit in series, upstream of the standard Siemens High-Speed Sensor Unit containing the standard $\mathrm{O}_{2}$ and total $\mathrm{CO}_{2}$ analysers (Fig. 1a). The integration of a methane $\left(\mathrm{CH}_{4}\right.$; $\mathrm{ABB}$ Automation $)$ and a hydrogen $\left(\mathrm{H}_{2}\right.$; Honeywell Analytics, Hegnau, Switzerland) analyser into our InCa system has been reported previously ${ }^{16}$. The ${ }^{12} \mathrm{CO}_{2}$ sensor has a range of $0-6000 \mathrm{ppm}$ which is appropriate in relation to total $\mathrm{CO}_{2}$ exchange in mice, since total $\mathrm{CO}_{2}$ ambient levels normally lie around $440 \mathrm{ppm}$ and can raise up to about 5000 ppm for single-housed adult mice (based on previous observations in our laboratory). The ${ }^{13} \mathrm{CO}_{2}$ sensor has a measuring range of 0-150 ppm suitable to measure natural ${ }^{13} \mathrm{CO}_{2}$ concentrations, which are estimated to be $5.5 \mathrm{ppm}$ in ambient air and $55 \mathrm{ppm}$ in mouse cages. Calibration of the equipment was done routinely with three gas mixtures (Linde Gas Benelux, Dieren, The Netherlands): zero (20.947\% $\mathrm{O}_{2}$, in $\mathrm{N}_{2}$, no other constituents), span 1 (98.8 ppm $\mathrm{H}_{2}$, in synthetic air), and span $2\left(0.521 \%\right.$ total $\mathrm{CO}_{2}, 450 \mathrm{ppm} \mathrm{CH}_{4}$, in $\mathrm{N}_{2}$ ). The zero calibration point was performed by flushing the zero gas mixture through the system for 10 min and assigning ADC signals their corresponding gas concentration values. The same procedure was repeated for the span calibration points using gas mixtures span 1 and span 2. The ${ }^{12} \mathrm{CO}_{2}$ and ${ }^{13} \mathrm{CO}_{2}$ span calibration points were set to 5153 and $57 \mathrm{ppm}$, respectively, based on the natural enrichment of atmospheric $\mathrm{CO}_{2}\left(1.1\right.$ atom\%) ${ }^{3}$. Cross-sensitivity between the ${ }^{12} \mathrm{CO}_{2}$ and ${ }^{13} \mathrm{CO}_{2}$ analysers is negligible. This calibration routine was performed before each experiment, with each experiment lasting for no more than a week, according to the stability of the ${ }^{12} \mathrm{CO}_{2}$ and ${ }^{13} \mathrm{CO}_{2}$ zero and span points of $<1 \%$ drift per week reported by the manufacturer. Raw data was acquired with a customised version of PhenoMaster software v.5.8.0 (TSE Systems), including ${ }^{12} \mathrm{CO}_{2}$ and ${ }^{13} \mathrm{CO}_{2}$ concentrations in ppm. Delta ppm values were obtained by subtracting reference cage values from mouse cage values at each time point, and these values were used for further calculations. Other operational settings and procedures have been described previously ${ }^{17}$. The overall performance of the newly extended system was first tested by measuring all gas concentrations over $5 \mathrm{~d}$ using empty cages. 

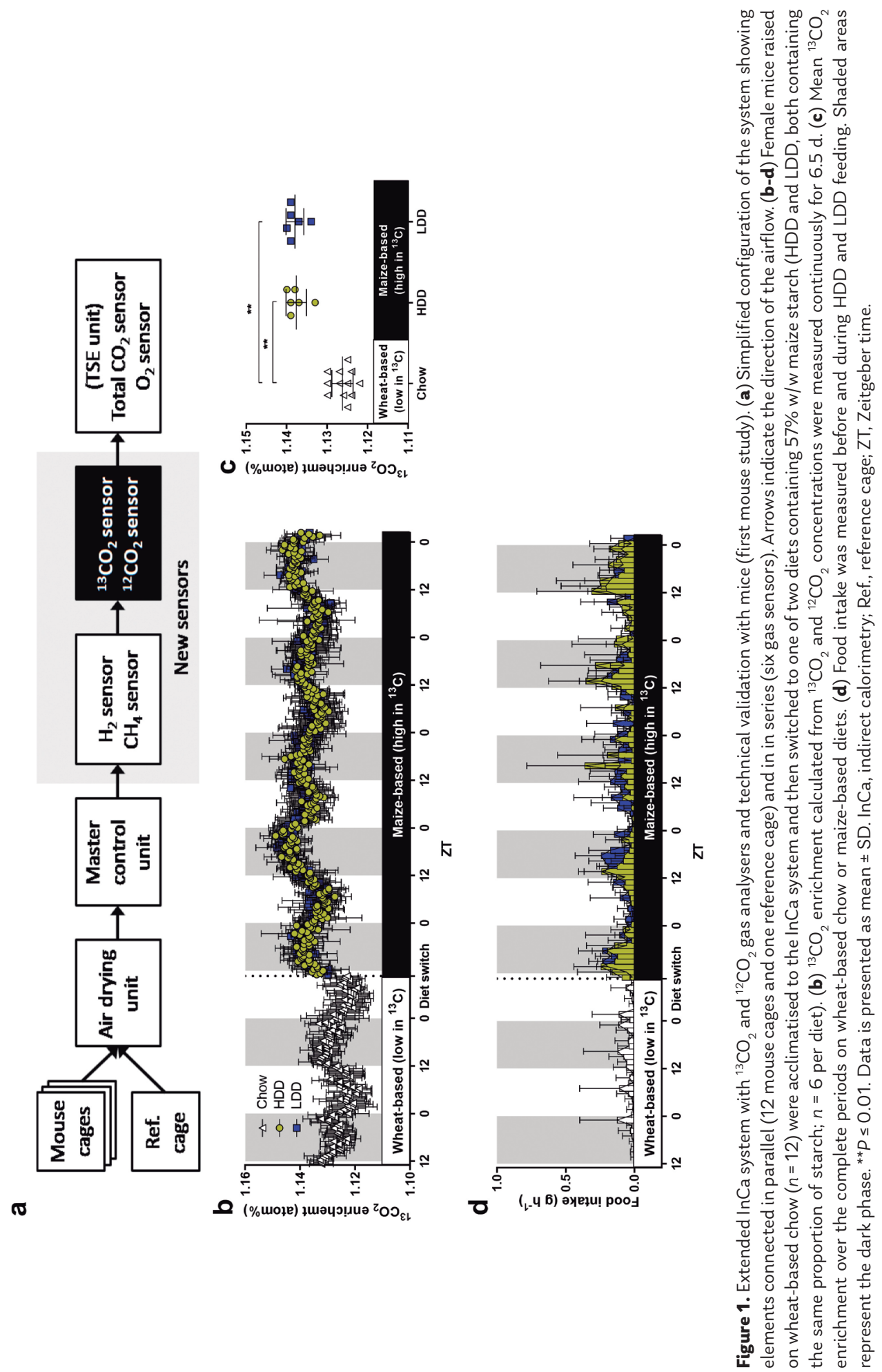
Composition of experimental diets

The highly digestible-starch diet (HDD) and the lowly digestible-starch diet (LDD) contained 20, 55, and 25 energy $\%$ protein, carbohydrate, and fat, respectively, and fulfilled the nutritional requirements for rodents according to AIN-93 ${ }^{18}$. The starches in HDD and LDD (569 $\mathrm{g} \mathrm{kg}^{-1}$ diet; Cargill, Sas van Gent, The Netherlands) were incorporated by Research Diet Services (Wijk bij Duurstede, The Netherlands) for the preparation of the pelleted diets. The high fat diet (HFD) contained 20,40, and 40 energy\% protein, carbohydrate, and fat, respectively. The exact composition of the experimental diets has been described in more detail elsewhere ${ }^{19}$.

Elemental analysis isotope ratio mass spectrometry (EA-IRMS)

The ${ }^{13} \mathrm{C}$ enrichments of the chow diet and the HDD and LDD were measured by EA-IRMS as published ${ }^{20}$. Briefly, pulverized samples were combusted at $1020{ }^{\circ} \mathrm{C}$ in the presence of oxygen to convert carbon into $\mathrm{CO}_{2}$, followed by separation for measurement of the ${ }^{13} \mathrm{C} /{ }^{12} \mathrm{C}$ ratio by EA-IRMS.

\section{Mouse experiments}

The experiments were approved by the Animal Experiment Committee of Wageningen University DEC2014085 and CCD/lvD 2017.w-0024.003, and performed in accordance with the European Union (EU) directives 86/609/EEC and 2010/63/EU, respectively. All mice (C57BL/6]RccHsd, Envigo, Horst, The Netherlands) were individually housed in Makrolon II cages with wood chips and enriched with wood shavings, at $23 \pm 1{ }^{\circ} \mathrm{C}, 50 \pm 5 \%$ humidity, on a $12 \mathrm{~h}$ light/dark cycle. Unless otherwise indicated, mice had ad libitum access to food and water.

Two mouse studies were conducted. The first study aimed to validate the newly incorporated ${ }^{13} \mathrm{CO}_{2}$ and ${ }^{12} \mathrm{CO}_{2}$ sensors using mice fed diets of variable natural ${ }^{13} \mathrm{C}$ enrichment. Ten-month old female mice $(n=12)$ raised on a chow diet (AM-II, AB Diets, Woerden, The Netherlands), with wheat as main ingredient and no declared content of C4 plant ingredients (1.078 atom $\%{ }^{13} \mathrm{C}$, EA-IRMS, see above), were weighed and acclimatised to the InCa environment for $48 \mathrm{~h}$. Mice were then switched to one of two maize-based semi-purified diets with $57 \% \mathrm{w} / \mathrm{w}$ starch: HDD (1.085 atom $\%{ }^{13} \mathrm{C}$, EA-IRMS, see above) or LDD (1.085 atom\% $\left.{ }^{13} \mathrm{C}, \mathrm{EA}-\mathrm{IRMS}\right)$. The allocation of HDD and LDD was randomised and known to the experimenter, and the body weight (BW) of these groups was similar. Air measurements continued for another $4.5 \mathrm{~d}$. Bedding volume was limited to approximately $200 \mathrm{ml}$ during InCa measurements to facilitate detection of voluntary locomotion by infrared beam breaks in the horizontal plane. All gas concentrations were measured continuously. Other data obtained from the animals in this experiment $\left(\mathrm{H}_{2}\right.$ production and gut microbiota composition) has been reported previously ${ }^{16}$.

The second study aimed at quantifying oxidation of ${ }^{13} \mathrm{C}$-labelled exogenous metabolic substrates in diet-induced obesity. Mice on a chow diet (Teklad Global Diet 2920, Envigo) were time-mated and their offspring cross-fostered within $24-48 \mathrm{~h}$ after birth. Female 
offspring $(n=48)$ were weaned at the end of postnatal week (PW) 3, stratified according to BW, and assigned to either HDD or LDD for 3 weeks; the experimenter was not blinded to these dietary treatments. Mice were then switched to a wheat-based HFD in PW 7 and continued on this diet until PW 15. Mice originally on HDD and LDD were initially treated as two experimental groups and, per group, were re-stratified by BW in PW 13 (prior to InCa and refeeding challenges in PW 14-15, see below) and again at the end of PW 15 before sacrifice in the fasted or postprandial condition (see below), to ensure the distribution of BW was similar across subgroups receiving the two differently labelled liquid mixed meals ( $n=24$ for either ${ }^{13} \mathrm{C}$ glucose or ${ }^{13} \mathrm{C}$ palmitate) and sacrificed in the two metabolic states ( $n=24$ fasted or postprandial). BW and food intake $(\mathrm{FI})$ were determined weekly. Body composition (BC; EchoMRI 100V, EchoMedical Systems, Houston, Texas, USA) was determined weekly (PW 4-6) or biweekly (PW 7-15), and directly before and after InCa runs. The RER response to refeeding with a liquid mixed meal, circulating fasting and postprandial glucose and insulin levels, and $24 \mathrm{~h}$ EE (Table S1 and Fig. S1) were not different between the mice originally on HDD or LDD. These metabolically very similar mice were then pooled into a group of in total 48 mice with a widely different fat mass (FM), and this combined group was used to investigate the metabolic response to the exogenous substrates of the liquid mixed meals and its correlation with FM.

InCa and refeeding challenge tests with liquid mixed meals

Individually housed mice (in batches of 12 per InCa run) were acclimatised to the InCa environment for approximately $24 \mathrm{~h}$. The following $24 \mathrm{~h}$ period was used for measurements of daily EE, RER, locomotor activity, and food and water intake. Sampling frequency for these basal gas measurements was every $20 \mathrm{~min}$. On the third day, 6 mice per batch, to facilitate a higher gas sampling frequency, were restricted to $1.1 \mathrm{~g}$ of food (HFD) $1 \mathrm{~h}$ before the dark phase (DP; ZT =11). The remaining 6 mice kept ad libitum access to food and water inside the InCa system. Twenty-four hours later $(Z T=11)$, the 6 cages with mice receiving restricted food were continuously measured at sample interval of $11 \mathrm{~min}$, and these mice received $\mathrm{a}^{13} \mathrm{C}$-labelled liquid mixed meal by oral gavage $(0.4 \mathrm{ml}$ per mouse, see below). The mice were continuously monitored in the extended InCa system, including ${ }^{13} \mathrm{CO}_{2}$ and ${ }^{12} \mathrm{CO}_{2}$ measurements, until the onset of the following light phase (LP; ZT = 0) for a total of $13 \mathrm{~h}$ upon ingestion of the liquid mixed meal, after which they regained ad libitum access to the HFD. On the fourth day, the same procedure (i.e. measurement following food restriction and a subsequent ${ }^{13} \mathrm{C}$-labelled liquid mixed meal) was repeated with the remaining 6 mice per batch in the InCa system. All of the mice received the same dose of liquid mixed meal. The meal contained a mixture of glucose and fat, representing $16.3 \pm 1.1 \%$ (mean $\pm \mathrm{SD}$ ) of daily energy expenditure, and contained either $a{ }^{13} \mathrm{C}$ glucose or a ${ }^{13} \mathrm{C}$ palmitate tracer (Table 1), allowing determination of glucose or palmitic acid oxidation specifically. 
Table 1. Composition of the liquid mixed meals.

\begin{tabular}{lcc}
\hline & ${ }^{13} \mathrm{C}$ Glucose & ${ }^{13}$ C Palmitate \\
\hline Unlabelled glucose $(\mathrm{mg})$ & $1358.4 \pm 0.7$ & $1401.3 \pm 1.1$ \\
\hline $\mathrm{U}^{13} \mathrm{C}$ Glucose $(\mathrm{mg})$ & $43.1 \pm 0.4$ & 0 \\
\hline Palm olein $(\mathrm{mg})$ & $977.5 \pm 2.3$ & $974.3 \pm 2.0$ \\
\hline Unlabelled sodium palmitate $(\mathrm{mg})$ & $23.9 \pm 0.2$ & 0 \\
\hline $\mathrm{U}^{13} \mathrm{C}$ Potassium palmitate $(\mathrm{mg})$ & 0 & $26.7 \pm 0.2$ \\
\hline Soy lecithin $(\mathrm{mg})$ & $8.1 \pm 0.9$ & $7.9 \pm 0.6$ \\
\hline Water $(\mathrm{mg})$ & $1501.7 \pm 2.8$ & $1501.0 \pm 1.1$ \\
\hline Density $\left(\mathrm{g} \mathrm{ml}{ }^{-1}\right)$ & $1.12 \pm 0.01$ & $1.11 \pm 0.02$ \\
\hline${ }^{13} \mathrm{C}$ content $(\mu \mathrm{mol})^{1}$ & $157.2 \pm 2.8$ & $7.16 \pm 0.12$ \\
\hline Energy $(\mathrm{kJ})^{1}$ & $7.19 \pm 0.09$ & $36.0 \pm 0.1$ \\
\hline Carbohydrate $(\mathrm{energy} \%)$ & $36.0 \pm 0.1$ & $63.9 \pm 0.1$ \\
\hline Fat (energy\%) & $64.0 \pm 0.1$ & 2.5 \\
\hline
\end{tabular}

${ }^{1}$ Amount per dose of $0.4 \mathrm{ml}$ of liquid mixed meal given by oral gavage. Data is presented as mean $\pm \mathrm{SD}$.

\section{Design and preparation of liquid mixed meals}

The liquid mixed meals were based on a recently developed drink used to measure the metabolic response to refeeding and health in humans ${ }^{21}$. However, as the focus was exclusively on exogenous glucose and fat oxidation, and therefore included corresponding metabolic tracers $\left({ }^{13} \mathrm{C}\right.$ glucose or ${ }^{13} \mathrm{C}$ palmitate), we omitted protein from the formulation. Palmitate was chosen as a fat tracer instead of labelled triglycerides or a mixture of fatty acids to circumvent possible fatty-acid-specific differences in absorption and oxidation. Sodium palmitate (Sigma-Aldrich, Missouri, State, USA) or D-glucose (Merck, Darmstadt, Germany) were partly replaced by either uniformly $(U){ }^{13} \mathrm{C}$-labelled potassium palmitate (98.8 atom\%, 98\% chemical purity; IsoLife, Wageningen, The Netherlands) or $\mathrm{U}-{ }^{13} \mathrm{C}$ D-glucose (99 atom\%, 98.8\% chemical purity; IsoLife), respectively, and mixed with soy lecithin (Emultop IP, Cargill, Hamburg, Germany) and ultrapure water. This mixture was then vortexed and microwaved until no visible lumps remained. Palm olein (Remia, Den Dolder, The Netherlands) was added and the aqueous and oily phases were integrated by vortexing and sonication until a homogenous emulsion was obtained. Fresh preparations were made $2 \mathrm{~h}$ before administration to the animals and remained stable. The overall composition of the labelled glucose and labelled palmitate liquid mixed meals is shown in Table 1.

Sacrifice in the fasted or postprandial state

Mice were food restricted (1.1 g HFD) for $16 \mathrm{~h}$, starting at ZT $=11$. Half of the mice was sacrificed in the post-absorptive state, and the other half was administered the liquid mixed meal with ${ }^{13} \mathrm{C}$ glucose by oral gavage and sacrificed after $45 \mathrm{~min}$ (postprandial state) by decapitation. Trunk blood was collected in MiniCollect serum tubes (Greiner Bio-One, Alphen aan de Rijn, The Netherlands). Serum was separated by centrifugation at $4{ }^{\circ} \mathrm{C}$ 
for $10 \mathrm{~min}$ at $3000 \times \mathrm{g}$, aliquoted, and stored at $-80{ }^{\circ} \mathrm{C}$. Glucose was measured in whole blood with a Freestyle glucose meter (Abbott Diabetes Care, Hoofddorp, The Netherlands) directly after sacrifice.

\section{Serum insulin measurements}

Fasting and postprandial serum insulin concentrations from animals sacrificed in PW 15 were determined with an Ultra-Sensitive Mouse Insulin ELISA Kit (ChrystalChem, Elk Grove Village, Illinois, USA) following the manufacturer's instructions. Samples were measured in duplicate.

Calculation of refeeding response ( $\triangle R E R)$, and total and exogenous substrate oxidation

The metabolic refeeding response $(\triangle R E R)$ was determined per individual animal as the change in RER from the fasting post-absorptive state (baseline over $1 \mathrm{~h}$ ) to postprandial $44 \mathrm{~min}$ after administering the liquid mixed meal, based on the median time when all mice achieved a postprandial RER peak. Total levels of glucose and fatty acid oxidation were calculated from $\mathrm{VO}_{2}$ and $\mathrm{VCO}_{2}$ obtained with the Siemens High-Speed Sensor Unit, using Péronnet \& Massicotte's table of non-protein RER ${ }^{22}$ and Weir's equation of $\mathrm{EE}^{23}$, as follows. Individual values for glucose and fatty acid oxidation (as \% of EE) were interpolated from the original table of Péronnet \& Massicotte, ranging from RER 0.7036 to 0.996 . These interpolated values of glucose or fatty acid utilisation (\% of EE) and the EE data [also obtained from the TSE system, based on Weir's equation: $\mathrm{EE}=\left(3.941 \times \mathrm{VO}_{2}\right)+\left(1.106 \times \mathrm{VCO}_{2}\right) ; \mathrm{kJ} \mathrm{min}^{-1}$, together with the energy equivalents of glucose and fatty acids $\left(16.18 \mathrm{~kJ} \mathrm{~g}^{-1}\right.$ and $40.76 \mathrm{~kJ} \mathrm{~g}^{-1}$, respectively ${ }^{22}$ ), were then used to calculate the rates of total glucose oxidation (TGO) and total fatty acid oxidation (TFO) in $\mathrm{mg} \mathrm{min}^{-1}$, according to the following equations:

$$
\begin{aligned}
& T G O=\frac{E E \times \% E E_{G O}}{16.18} \\
& T F O=\frac{E E \times \% E E_{F O}}{40.76}
\end{aligned}
$$

Rates of exogenous substrate oxidation (ESO; i.e. oxidation of ingested glucose and palmitate in the liquid mixed meal based on the ${ }^{13} \mathrm{C}$ tracers) were calculated using the following two equations:

$$
\begin{gathered}
a t \%{ }^{13} \mathrm{CO}_{2}=\frac{{ }^{13} \mathrm{CO}_{2}}{{ }^{13} \mathrm{CO}_{2}+{ }^{12} \mathrm{CO}_{2}} \times 100 \\
\operatorname{ESO}\left(\mathrm{mg} \times \mathrm{min}^{-1}\right)=\frac{a t{ }^{13} \mathrm{CO}_{2}(t)-a t{ }^{13} \mathrm{CO}_{2}(t 0)}{a t \%^{13} C_{S}-a t 0^{13} \mathrm{CO}_{2}(t 0)} \times \frac{\mathrm{VCO}_{2}(t)}{22.2966 \times C_{\text {tracer }}}
\end{gathered}
$$

In equations (3) and (4), at $\%{ }^{13} \mathrm{CO}_{2}$ is the ${ }^{13} \mathrm{C}$ enrichment in expired $\mathrm{CO}_{2}$ in atom\% calculated from gas concentrations (delta ppm). In equation (4), time $t_{0}$ represents the baseline measurement over $1 \mathrm{~h}$ before administration of the liquid mixed meals and $\mathrm{t}$ represents any subsequent time point. The calculated ${ }^{13} \mathrm{C}$ enrichment of the whole 
substrate pool ingested (either unlabelled glucose plus ${ }^{13} \mathrm{C}$ glucose, or unlabelled palmitate plus ${ }^{13} \mathrm{C}$ palmitate) is represented by at $\%{ }^{13} \mathrm{C}$, assuming a natural terrestrial ${ }^{13} \mathrm{C}$ enrichment of 1 atom\% (ref. 4), 100\% chemical purity, and following the fatty acid composition of palm olein from literature ${ }^{24} . \mathrm{VCO}_{2}$ is the production rate of $\mathrm{CO}_{2}$ obtained using the summed concentrations of ${ }^{13} \mathrm{CO}_{2}$ and ${ }^{12} \mathrm{CO}_{2}$ measured by the URAS26 module multiplied by the air flow (constant). The molecular weight of the tracee ( $\mathrm{MW}_{\text {tracee }}$ glucose or palmitic acid) and the volume occupied by $1 \mathrm{~mol}$ of $\mathrm{CO}_{2}$ in STPD $(22.2966 \mathrm{l})$ are based on Péronnet \& Massicotte ${ }^{22}$. The number of labelled carbons per mol of tracer $\left(C_{\text {tracer }}\right)$ is 6 for $\mathrm{U}^{13} \mathrm{C}$ glucose and 16 for $U-{ }^{13} \mathrm{C}$ palmitate. Of note, exogenous fat oxidation (EFO) represents only the oxidation of palmitic acid ingested (both labelled and unlabelled), thus oxidation of other fatty acids in the liquid mixed meal (mainly oleic acid and linoleic acid) is not accounted for.

Additionally, a dietary acetate recovery factor (dARF) was implemented in our calculations giving rise to an alternative ESO calculation, equation (5). The dARF is a factor suggested to be used to correct for ${ }^{13} \mathrm{C}$ sequestration based on studies in normal weight and obese humans ${ }^{25,26}$, but has not been validated in mice. In detail, the animal with the highest FM and the animal with the lowest FM were assigned the dARF of obese (0.453) and normal weight humans (0.506), respectively, and the dARF of the remaining animals was interpolated by linear regression and applied to equation (4):

$$
\operatorname{ESO}\left(\mathrm{mg} \times \min ^{-1}\right)=\frac{a t \%^{13} \mathrm{CO}_{2}(t)-a t \%^{13} \mathrm{CO}_{2}(t 0)}{a t 0^{13} C_{S}-a t 0^{13} \mathrm{CO}_{2}(t 0)} \times \frac{V{ }^{2} O_{2}(t)}{22.2966 \times C_{\text {tracer }} \times d A R F}
$$

\section{Statistical analysis}

Each individual mouse was considered an experimental unit. Normal distribution of the data was tested with the D'Agostino and Pearson omnibus test; non-normally distributed data were log-transformed and retested for normality. The difference in natural ${ }^{13} \mathrm{C}$ enrichment on wheat- us maize-based diets was analysed with the Kruskal-Wallis test with Dunn's multiple comparison test. Correlations were performed by Pearson correlation (normallydistributed data) or Spearman correlation (non-normally distributed data). Technical errors occurred in oral gavage in two animals, therefore this data was not included for analyses of postprandial metabolic outcomes. All statistical analyses and data visualisation were performed in Prism v.5.04 (GraphPad, San Diego, California, USA). Data is presented as mean \pm SD (normally distributed data) or median and range (non-normally distributed data), and statistical significance was set at $P<0.05$. 


\section{Results}

Integration of ${ }^{13} \mathrm{CO}_{2}$ and ${ }^{12} \mathrm{CO}_{2}$ sensors into the InCa system and analysis of natural dietary ${ }^{13} \mathrm{C}$ enrichment

We first evaluated the overall performance of the newly integrated ${ }^{12} \mathrm{CO}_{2}$ and ${ }^{13} \mathrm{CO}_{2}$ sensors into the InCa system (Fig. 1a) with a $5 \mathrm{~d}$ test with empty cages to measure diurnal fluctuations in ambient air. Median ambient levels during this test were $5.30 \mathrm{ppm}{ }^{13} \mathrm{CO}_{2}(4.42,6.08$; range) and $462.8 \mathrm{ppm}{ }^{12} \mathrm{CO}_{2}$ (449.7, 535.2; range), respectively. This corresponded to a median of 1.130 atom $\%{ }^{13} \mathrm{CO}_{2}(0.960,1.159$; range).

After these initial tests, female mice raised on wheat-based chow (a C3 plant, thus low in ${ }^{13} \mathrm{C}$ ) were placed into the InCa system and ${ }^{13} \mathrm{CO}_{2},{ }^{12} \mathrm{CO}_{2}$, and other gas concentrations were recorded continuously for $48 \mathrm{~h}$ (Fig. 1b). Once animals were acclimatised, the wheatbased chow was exchanged for one of two maize-based semi-purified diets, a highly digestible-starch diet (HDD) and a lowly digestible-starch diet (LDD), each containing the same amount of maize starch (a $\mathrm{C} 4$ plant, thus naturally high in ${ }^{13} \mathrm{C}$ ). Oscillations in ${ }^{13} \mathrm{CO}_{2}$ enrichment followed a circadian pattern, reaching the lowest levels during the light phase (LP) and highest levels during the dark phase (DP; Fig. 1b); this pattern was similar to the food intake (FI) pattern (Fig. 1d). Upon the switch to a maize-based diet, overall ${ }^{13} \mathrm{CO}_{2}$ enrichment levels were significantly higher $(P=0.0002)$, independent of type of maize starch used, with a mean difference of 0.011 and 0.012 atom\% for HDD and LDD, respectively, compared to chow (Fig. 1c). The ${ }^{13} \mathrm{C}$ enrichment of the diets measured by elemental analysis isotope ratio mass spectrometry (EA-IRMS) was 1.078 atom \% for chow and 1.085 atom $\%$ for both HDD and LDD, thus a natural enrichment difference of 0.008 atom\%, which is close to the observed ${ }^{13} \mathrm{CO}_{2}$ differences in the expired air. Additionally, analysis of a separate group of mice that remained on chow instead of switching to HDD or LDD confirmed that the increase in mean ${ }^{13} \mathrm{CO}_{2}$ enrichment was driven mainly by dietary ${ }^{13} \mathrm{C}$ enrichment and less so by increased FI (Fig. S2). Finally, to assess the functionality of the newly incorporated sensors as part of conventional respirometry, we recalculated $\mathrm{EE}$ by substituting total $\mathrm{VCO}_{2}$ values obtained with a single sensor with the sum of $\mathrm{V}^{13} \mathrm{CO}_{2}$ and $\mathrm{V}^{12} \mathrm{CO}_{2}$ values obtained from two sensors. Recalculated and original $24 \mathrm{~h}$ EE values were almost identical (41.03 \pm 0.78 us $41.03 \pm 0.77 \mathrm{~kJ} \mathrm{~d}^{-1}$, respectively, Student's t-test $P=0.994$; linear regression, $\left.Y=0.9993 X+0.0002 ; r^{2}=0.999\right)$.

\section{Refeeding metabolic response in diet-induced obesity}

After 9 weeks on a high-fat-diet (HFD), mice that were previously fed LDD us HDD for 3 weeks had a slightly higher fat mass (FM) and gained more FM over the period on HFD, however, BW was not significantly different between the groups (Table S1). The total group of 48 mice on HFD had a FM ranging from $5.52 \mathrm{~g}$ up to $15.88 \mathrm{~g}$ (288\%; Table 2) and was metabolically fairly homogeneous, regardless of prior LDD or HDD feeding (Table $\mathrm{S} 1$ and Fig. S1). The metabolic response to refeeding was tested using ${ }^{13} \mathrm{C}$-labelled liquid mixed meals, and the data derived from the ${ }^{13} \mathrm{CO}_{2}$ and ${ }^{12} \mathrm{CO}_{2}$ sensors allowed us to detect exogenous tracer $\left({ }^{13} \mathrm{C}\right)$ as a marker for the total specific substrate, simultaneously with overall substrate oxidation based on $\mathrm{O}_{2}$ consumption and total $\mathrm{CO}_{2}$ production. Baseline 
RER values before administration of the liquid mixed meal were $0.70 \pm 0.01$ (mean $\pm S D$; Fig. 2). The median peak RER (glucose oxidation) was achieved after $44 \mathrm{~min}$ (22, 88; range) upon feeding, and values declined thereafter until full fat oxidation ${ }^{22}$ was reached again after approximately $140 \mathrm{~min}$. The refeeding RER response, defined as the increase from baseline RER to RER at $44 \mathrm{~min}(\triangle \mathrm{RER}$, Table 2), was negatively correlated to FM (measured directly prior to InCa measurements; Fig. 2c). In line, also BW (Spearman $r=-0.386$, $P=0.0074 ; n=47)$ and $L M\left(r^{2}=0.245, P=0.0004 ; n=47\right)$ were negatively correlated to $\triangle R E R$. Serum insulin levels 45 min post-prandially were also negatively correlated to $\triangle R E R$ in a subgroup of animals that were fasted and challenged again with the liquid mixed meal before sacrifice $\left(r^{2}=0.421, P=0.0011 ; n=22\right)$.

Table 2. Metabolic characteristics of the mice after HFD-feeding for nine weeks (second mouse study).

\begin{tabular}{|c|c|}
\hline Parameter & Outcome \\
\hline $\mathrm{BW}(\mathrm{g})^{1}$ & $31.57(24.82,38.22)$ \\
\hline $\mathrm{FM}(\mathrm{g})^{1}$ & $10.21(5.52,15.88)$ \\
\hline $\mathrm{LM}(\mathrm{g})^{1}$ & $19.89(17.68,21.64)$ \\
\hline FM gain $(g)^{2}$ & $8.66(4.41,14.06)$ \\
\hline FI $(g)^{2}$ & $188.38(161.67,226.33)$ \\
\hline $24 \mathrm{~h} \mathrm{EE}\left(\mathrm{kJ} \mathrm{d}^{-1}\right)^{3}$ & $44.24(38.66,51.45)$ \\
\hline $24 \mathrm{~h} \mathrm{RER}^{3}$ & $0.81(0.74,0.89)$ \\
\hline$\Delta$ RER $\left(\operatorname{RER}_{44}-\mathrm{RER}_{0}\right)^{3}$ & $0.16(0.10,0.20)$ \\
\hline Fasting glucose $\left(\mathrm{mmol} \mathrm{l}^{-1}\right)^{1}$ & $6.0(4.9,7.2)$ \\
\hline Fasting insulin $\left(\mathrm{ng} \mathrm{ml}^{-1}\right)^{1}$ & $2.65(0.85,8.95)$ \\
\hline Postprandial glucose $\left(\mathrm{mmol} \mathrm{l}^{-1}\right)^{1}$ & $6.9(3.3,9.1)$ \\
\hline Postprandial insulin $\left(\mathrm{ng} \mathrm{ml}^{-1}\right)^{1}$ & $4.40(1.47,9.22)$ \\
\hline \multicolumn{2}{|c|}{$\begin{array}{l}\text { Measured in PW } 15 \text { (end of HFD). }{ }^{2} \text { Measured from PW } 7 \text { (start of HFD) to } \\
\text { PW 15. }{ }^{3} \text { Measured in PW } 14-15 . \text { HFD, high-fat diet; BW, body weight; FM, fat } \\
\text { mass; LM, lean mass; FI, food intake; EE, energy expenditure; RER, respiratory } \\
\text { exchange ratio (mean of } 24 \text { h). Data is presented as means and range, } n \\
48 \text { (except } \triangle \text { RER, } n=47 \text {; fasting glucose and insulin } n=24 \text {; and postprandial } \\
\text { glucose and insulin, } n=23 \text { ). }\end{array}$} \\
\hline
\end{tabular}

Oxidation of exogenous (tracer) glucose and fat

The liquid mixed meal was enriched with metabolic ${ }^{13} \mathrm{C}$ tracers for glucose or palmitate to investigate dietary fuel partitioning between storage and oxidation, continuously and in real time. Thus, all animals received a liquid mixed meal of identical macronutrient composition, but containing either ${ }^{13} \mathrm{C}$ glucose or ${ }^{13} \mathrm{C}$ palmitate, and exhaled ${ }^{13} \mathrm{CO}_{2}$ was measured to reflect the oxidation of tracer and total glucose and palmitate contained in 
a

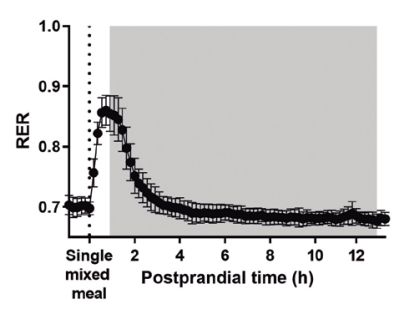

b

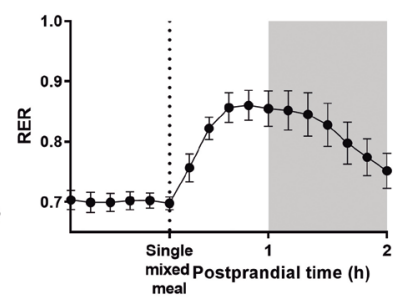

C

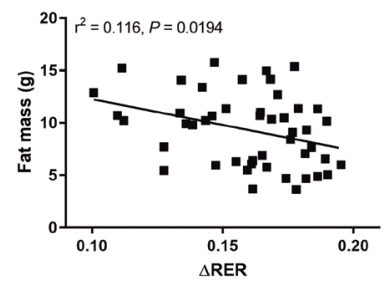

Figure 2. Refeeding metabolic response to a liquid mixed meal and its relation to body fat mass (FM) in mice fed a HFD for nine weeks (second mouse study). (a) Respiratory exchange ratio (RER) was measured in PW 14-15 after gavage of a single liquid mixed meal containing 36 energy $\%$ glucose and 64 energy $\%$ fat $(n=47)$. Data is obtained from conventional indirect calorimetry measurements $\left(\mathrm{VO}_{2}\right.$ and $\left.\mathrm{VCO}_{2}\right)$. Data is presented as mean \pm SD. Shaded areas represent the dark phase. (b) Data from panel (a), emphasizing the early postprandial $2 \mathrm{~h}$ period. (c) Refeeding metabolic response ( $\triangle R E R$ ), measured as the increase from baseline RER to RER at $44 \mathrm{~min}$ postprandial, correlates negatively with FM. PW, postnatal week.

the meal. Concentrations of ${ }^{13} \mathrm{CO}_{2}$ and ${ }^{12} \mathrm{CO}_{2}$ were first computed as ${ }^{13} \mathrm{CO}_{2}$ enrichment to characterise exogenous fuel oxidation qualitatively (Fig. 3). The label in the ${ }^{13} \mathrm{C}$ glucose liquid mixed meal appeared in breath quickly in a single peak at $79 \pm 12$ min (mean $\pm S D$ ), and ${ }^{13} \mathrm{CO}_{2}$ enrichment fell almost back to baseline by the end of the $13 \mathrm{~h}$ post-meal period (final enrichment $1.159 \pm 0.012$ atom\%, mean \pm SD; Fig. 3a). In contrast, the label in the ${ }^{13} \mathrm{C}$ palmitate liquid mixed meal appeared later and at a slower rate, generally peaking around $4 \mathrm{~h}$ (with individual animals showing multiple peaks), and continued to appear by the end of the $13 \mathrm{~h}$ period (final enrichment $1.242 \pm 0.038$ atom $\%$, mean $\pm \mathrm{SD}$; Fig. 3c). The underlying concentrations of ${ }^{13} \mathrm{CO}_{2}$ and ${ }^{12} \mathrm{CO}_{2}$ used to calculate ${ }^{13} \mathrm{CO}_{2}$ enrichments are shown in Fig. 3b,d.

To quantify and dissect exogenous substrate oxidation rates, ${ }^{13} \mathrm{CO}_{2}$ enrichment data was combined with $\mathrm{VCO}_{2}$ production rates and compared to total substrate oxidation, i.e. endogenous and exogenous, obtained from conventional InCa equations (Fig. 4). Since the substrate oxidation kinetics of HDD and LDD mice were almost identical (Fig. S1), the data was further analysed and plotted as one group. Exogenous glucose oxidation (EGO) and total glucose oxidation (TGO) rates followed in general similar kinetics (Fig. 4a,b). However, EGO achieved a later peak compared to TGO, with a median peak at 77 min $(44,132$; range; $n=23)$ and $66 \min (33,99$; range; $n=47)$, respectively (Mann Whitney, $P=0.0002$; Fig. 4a,b). Maximal EGO and TGO were $0.934 \pm 0.212$ and $1.211 \pm 0.213 \mathrm{mg} \mathrm{min}^{-1}$ (mean \pm SD), respectively. Thereafter EGO and TGO started to decline until they became negligible, with EGO generally remaining slightly higher than TGO (Fig. 4a), likely due to underestimation of total glucose oxidation (see Discussion). In contrast to glucose oxidation, exogenous fat oxidation (EFO), accounting only for palmitic acid, and total fat oxidation (TFO) rates showed clearly different kinetics (Fig. 4c,d). Immediately after administration of the liquid mixed meal, EFO started to rise while TFO was abruptly suppressed (Fig. 4c,d). Later TFO returned back to baseline around $2 \mathrm{~h}$ and EFO peaked around $4 \mathrm{~h}$ postprandial (Fig. 4c,d). 
a

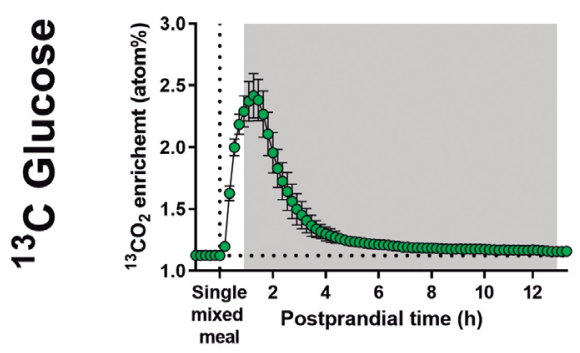

C

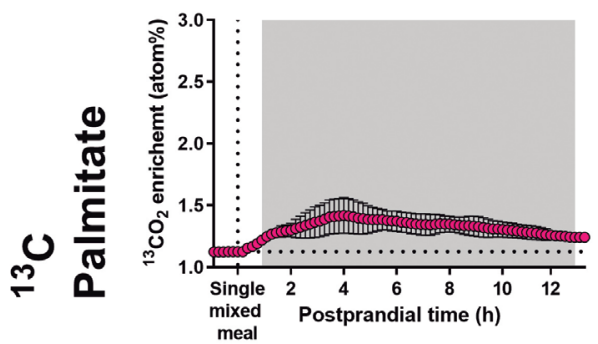

b

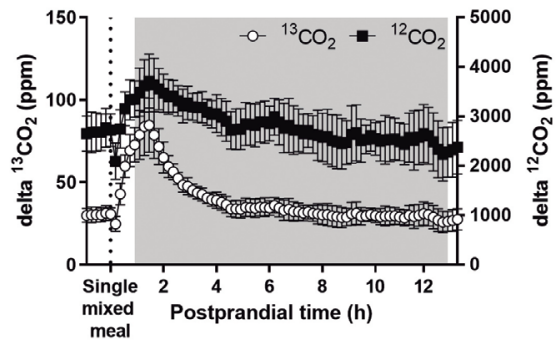

d

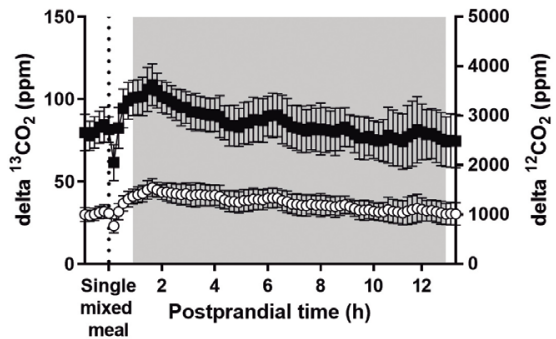

Figure 3. Kinetics of instantaneous ${ }^{13} \mathrm{CO}_{2}$ enrichment measured by extended InCa after gavage of a single liquid mixed meal containing ${ }^{13} \mathrm{C}$-labelled tracers, in mice fed a HFD for nine weeks (second mouse study). (a) ${ }^{13} \mathrm{CO}_{2}$ enrichment after ingestion of the ${ }^{13} \mathrm{C}$ glucose liquid mixed meal followed for $13 \mathrm{~h}(n=23)$, calculated from ${ }^{13} \mathrm{CO}_{2}$ and ${ }^{12} \mathrm{CO}_{2}$ concentrations. (b) ${ }^{13} \mathrm{CO}_{2}$ (left y-axis) and ${ }^{12} \mathrm{CO}_{2}$ (right y-axis) concentrations used to calculate ${ }^{13} \mathrm{CO}_{2}$ enrichments as shown in panel (a), expressed as the difference (delta) from gas concentrations measured in mouse-occupied cages minus gas concentrations in the reference cage (i.e. gas production). (c) ${ }^{13} \mathrm{CO}_{2}$ enrichment after ingestion of the ${ }^{13} \mathrm{C}$ palmitate liquid mixed meal $(n=24)$. (d) ${ }^{13} \mathrm{CO}_{2}$ (left y-axis) and ${ }^{12} \mathrm{CO}_{2}$ (right $y$-axis) concentrations used to calculate ${ }^{13} \mathrm{CO}_{2}$ enrichments as shown in panel (c). The horizontal dotted lines indicate baseline ${ }^{13} \mathrm{CO}_{2}$ enrichment. Shaded areas represent the dark phase. Data is presented as mean $\pm \mathrm{SD}$. PW, postnatal week.

Maximal EFO and TFO were $0.047 \pm 0.013$ and $0.936 \pm 0.069 \mathrm{mg} \mathrm{min}^{-1}($ mean \pm SD), respectively. Both EFO and TFO tended to decrease towards the end of the measurements at $13 \mathrm{~h}$ but remained above the baseline (Fig. 4c).

Cumulatively, EGO was $111.7 \mathrm{mg}(97.5,122.8$; median and range) and TGO $103.5 \mathrm{mg}$ (68.5, 159; median and range) during the complete post-meal measurement period, from consumption of the liquid mixed meal $1 \mathrm{~h}$ before the DP and until the end of the DP (13 h in total). This amount of exogenous glucose oxidised corresponded to $69.6 \%(61.3,76.6$; median and range) of the total dose administered with the liquid mixed meal (Fig. 4e,f). In comparison, EFO was $16.4 \mathrm{mg}$ (9.6, 18.7; median and range) and TFO $511.0 \mathrm{mg}$ (404.2, 635.4; median and range) over the whole period. For exogenous palmitate oxidised, this amount corresponded to $47.6 \%$ (27.7, 52.6; median and range) of the oral dose (Fig. 4e,f). The percentage of dose oxidised was more variable for exogenous palmitate $(C V=13.4 \%)$ than for exogenous glucose ( $C V=5.1 \%)$. 

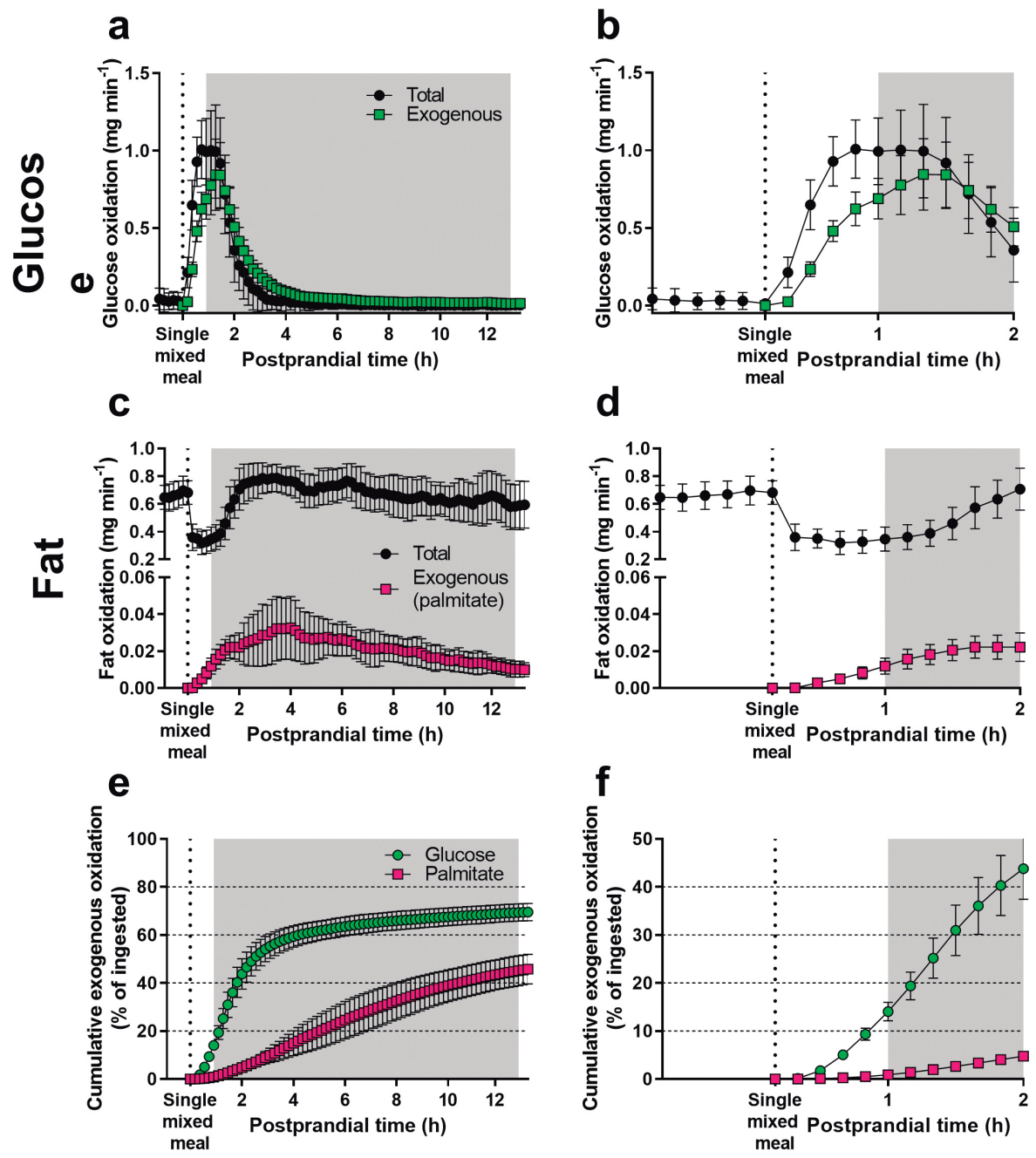

Figure 4. Kinetics of oxidation of total and exogenous metabolic substrates after gavage of a labelled liquid mixed meal, in mice fed a HFD for nine weeks (second mouse study). (a) Instantaneous total $(n=47)$ and exogenous $(n=23)$ glucose oxidation after administration of the ${ }^{13} \mathrm{C}$ glucose liquid mixed meal until the end of the measurements. (b) Data from panel (a), emphasizing the early $2 \mathrm{~h}$ postprandial period. (c) Instantaneous total fat oxidation $(n=47)$ and exogenous palmitate oxidation $(n=24)$ after administration of the ${ }^{13} \mathrm{C}$ palmitate liquid mixed meal. (d) Data from panel (c), emphasizing the early $2 \mathrm{~h}$ postprandial period. (e) Cumulative exogenous glucose $(n=23)$ and palmitate oxidation $(n=24)$ after administration of the labelled liquid mixed meals until the end of the measurements. (f) Data from panel (e), emphasizing the early $2 \mathrm{~h}$ postprandial period. Exogenous substrate oxidation data is calculated from conventional $\ln \mathrm{Ca}$ data $\left(\mathrm{VCO}_{2}\right)$ and ${ }^{13} \mathrm{CO}_{2}$ and ${ }^{12} \mathrm{CO}_{2}$ data. Shaded areas represent the dark phase. Data is presented as mean $\pm \mathrm{SD}$. InCa, indirect calorimetry; PW, postnatal week. 
Exogenous substrate oxidised in relation to body composition

We then attempted to explain the variation in exogenous fuel selection in diet-induced obese mice by correlating cumulative oxidation data with FM (determined directly before InCa). TFO was positively correlated to FM (0-2 h: $\mathrm{r}^{2}=0.438, P<0.0001 ; 2-13 \mathrm{~h}: \mathrm{r}^{2}=0.467$, $P<0.0001 ; n=47)$, while EGO, TGO, and EFO did not reach a statistically significant correlation, neither during the early postprandial period from 0-2 h, nor from 2-13 h after ingestion of the liquid mixed meal.

Although LM showed a narrow range of values (17.68, 21.64; Table 2), it correlated positively with $\mathrm{FM}\left(\mathrm{r}^{2}=0.349, P<0.0001 ; n=48\right)$, and thus differences in total oxidative tissue may explain the correlations of substrate oxidation with FM. Therefore, oxidation data were also expressed relative to LM. Correlations of EGO with FM remained non-significant (Fig. 5a,b), and TGO correlated negatively with FM only during the early postprandial period ( $0-2 \mathrm{~h}$ : $\left.r^{2}=0.101, P=0.0296 ; 2-13 h: r^{2}=0.039, P=0.1861 ; n=47\right)$. Positive correlations of TFO with FM (0-2 h: $\left.r^{2}=0.355, P<0.0001 ; 2-13 h: r^{2}=0.2838, P=0.0001 ; n=47\right)$ remained significant. Moreover, after accounting for LM, EFO correlated negatively with FM (2-13 h: $\left.r^{2}=0.165, P=0.0488 ; n=24\right)$, only during the late postprandial and post-absorptive period (Fig. 5c,d).

Expressed relative to TFO, EFO was negatively correlated to FM (O-2 $h: r^{2}=0.262, P=0.0105$; 2-13 h: $\left.r^{2}=0.350, P=0.0023 ; n=24 ; F i g .5 e, f\right)$. Additionally, EFO relative to TFO was positively correlated with $\triangle$ RER $\left(0-2 h r^{2}=0.225, P=0.0192 ; 2-13 h: r^{2}=0.224, P=0.0194 ; n=24\right.$; Fig. $5 \mathrm{~g}, \mathrm{~h}$ ), an unsuspected outcome since the refeeding response is generally qualified by a switch to glucose oxidation and concomitant suppression of fat oxidation.

In metabolic tracer studies with humans, particularly when using isotopically labelled fatty acids, it is important to account for label sequestration in the body bicarbonate pool and in exchange reactions in the TCA cycle, and this can be achieved by applying an acetate correction factor to the oxidation calculation $\mathrm{s}^{27}$. When the labelled fatty acids are ingested, this factor is called the dietary acetate recovery factor (dARF) and its value depends on whole-body adiposity and fasting insulin levels ${ }^{25}$. To our knowledge, a dARF is yet to be determined for mice. Since ${ }^{13} \mathrm{C}$ label sequestration from palmitate could also depend on FM in mice and lead to potentially wrong interpretations of exogenous oxidation data, we tested the potential consequences of applying this factor. In view of a lack of dARF in mice, we applied the dARF known for normal-weight and obese humans (13.3, $31.3 \mathrm{~kg}$ FM; range) to individual mice, assuming a linear relationship between FM and the dARF. Including a dARF in the calculations of EFO led to much higher label recoveries, with a median of $99.0 \%$ (59.2, 116.0; range) exogenous palmitate oxidation of dose administered. After accounting for label sequestration in this way, EFO relative to LM was no longer significantly correlated to FM. However, EFO relative to TFO remained negatively correlated to FM (0-2 h: $\mathrm{r}^{2}=0.188$, $\left.P=0.0345 ; 2-13 h: r^{2}=0.224, P=0.0194 ; n=24\right)$, and also remained positively correlated to $\triangle$ RER (0-2 h: $\left.r^{2}=0.211, P=0.0241 ; 2-13 h: r^{2}=0.195, P=0.0306 ; n=24\right)$. 
a

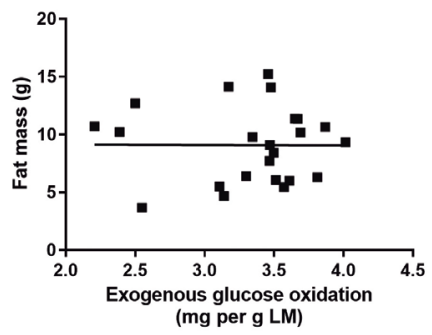

C

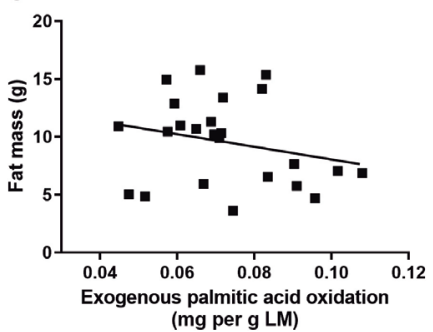

e

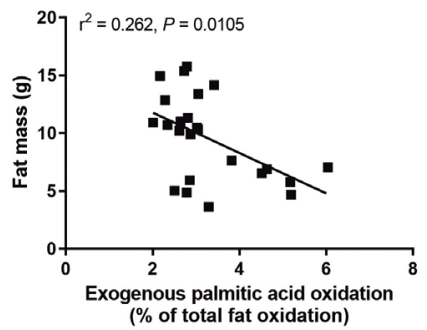

g

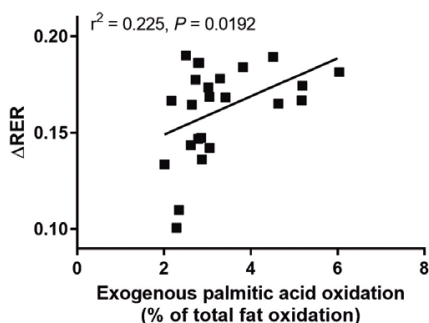

b

$2-13 \mathrm{~h}$

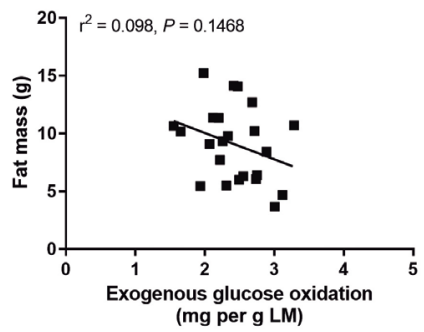

d $\quad 2-13 \mathrm{~h}$
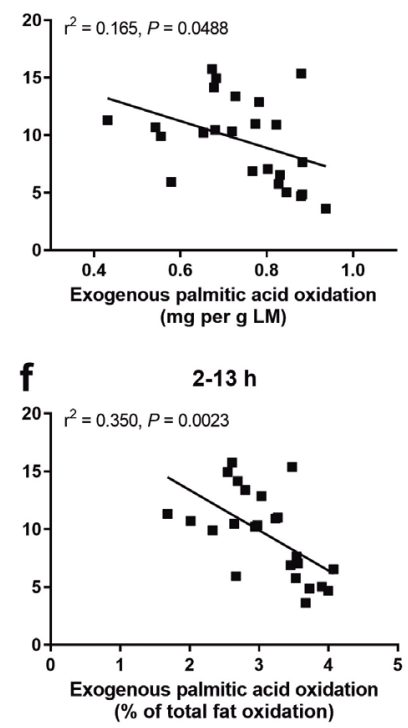

$\mathbf{h}$ 2-13 h

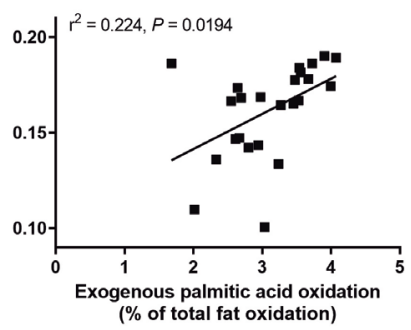

Figure 5. Correlation analysis between cumulative exogenous glucose or fat oxidation after gavage of a labelled liquid mixed meal and body fat mass and $\triangle R E R$, in mice fed a HFD for nine weeks (second mouse study). Exogenous glucose oxidation from 0-2 $\mathrm{h}$ (a) or 2-13 h postprandial (b) expressed per unit of LM is not significantly correlated to FM. Exogenous palmitic acid oxidation from 0-2 h postprandial expressed per unit of LM is not significantly correlated to FM (c), but it is negatively correlated to FM 2-13 h after ingestion of the liquid mixed meal (d). Exogenous palmitic acid oxidation expressed as a percentage of total fat oxidation correlates negatively with FM, both from 0-2 $\mathrm{h}$ postprandial (e) and from 2-13 h after ingestion of the liquid mixed meal (f). Exogenous palmitic acid oxidation relative to total fat oxidation correlates positively with $\triangle \mathrm{RER}$, both from $0-2 \mathrm{~h}$ postprandial (g) and from 2-13 $h$ after ingestion of the liquid mixed meal $(\mathbf{h}) . n=24$. PW, postnatal week. 


\section{Discussion}

We have incorporated two new gas sensors, for ${ }^{13} \mathrm{CO}_{2}$ and ${ }^{12} \mathrm{CO}_{2}$, into a commercial indirect calorimetry (InCa) system for mice. Using this extended InCa system, we were able to detect long-term continuous changes in ${ }^{13} \mathrm{CO}_{2}$ enrichment based on the natural ${ }^{13} \mathrm{C}$ content of the diet (wheat-based us maize-based diets) and to analyse and dissect the use of exogenous or endogenous body fuels (feeding us fasting conditions). Furthermore, the extended system was particularly suitable for real-time and continuous quantification of exogenous substrate oxidation using ${ }^{13} \mathrm{C}$-labelled ingredients. Combined with conventional InCa, providing mice a liquid mixed tracer meal revealed that with increased FM a blunted switch to glucose oxidation was accompanied with a decreased utilisation of exogenous dietary palmitate. Thus, extended $\ln \mathrm{Ca}$ with ${ }^{13} \mathrm{CO}_{2}$ and ${ }^{12} \mathrm{CO}_{2}$ sensors provided a deeper understanding of metabolic phenotypes.

Quantifying the oxidative disposal of exogenous metabolic substrates has been used in many human metabolic studies ${ }^{3}$. However in mice, to the best of our knowledge, only one published study quantified oxidation of glucose and fat ingested in a meal using stable isotopic tracers ${ }^{14}$. Due to the choice of different tracers $\left({ }^{13} \mathrm{C}\right.$ glucose replacing sucrose, and ${ }^{13} \mathrm{C}$ trioleate replacing soybean oil) and the lack of data on dose recovery percentages ${ }^{14}$, it is not possible to compare our conclusions regarding quantification of oxidised substrates. Comparison with other species and protocols is not easy, given the diversity in metabolic rates, doses, tracers, study durations, and other experimental conditions. Nevertheless, our results are consistent with the general notion that ingested fats are less readily oxidised than carbohydrates ${ }^{5}$, and with the observation that maximal oxidation rates of ingested palmitate were one order of magnitude smaller than for glucose, as was investigated in another rodent, Phodopus sungorus ${ }^{8}$. At the same time, the cumulative oxidation of ingested palmitate was highly variable compared to that of ingested glucose ( $C V=13.4 \%$ us $5.1 \%$ for palmitate and glucose, respectively). Together, these data reinforce the concept that glucose balance is more tightly controlled than fat balance, at least in the shortterm $^{28,29}$. Arguably, glucose oxidation is the most straightforward postprandial oxidation fuel strategy, as fat oxidation has been shown to depend on chain length and degree of saturation $^{30-33}$ and even physical structure ${ }^{11,34}$.

Using ${ }^{13} \mathrm{CO}_{2}$ and ${ }^{12} \mathrm{CO}_{2}$ sensors in combination with InCa allows for a more refined interpretation of the capacity to adjust substrate oxidation to its availability, which is also referred to as metabolic flexibility ${ }^{35}$. We $\mathrm{e}^{17,19,36-38}$ and others ${ }^{9,39}$ have used this concept as a strategy for metabolic phenotyping in mice by measuring the RER. However, it is unclear to what extent this adaptation to available substrates involves the oxidation of exogenous substrates per se, since this question cannot be answered calculating RER alone. As could be expected as part of an obese metabolic phenotype, a blunted RER response to refeeding was correlated to increased body fat mass in our mice (Fig. 2c). Remarkably, this was only reflected in the total amount of glucose oxidised in the early postprandial period, while the total amount of ingested glucose that became oxidised was not related to fat mass. This could suggest that hepatic uptake and regulation of ingested glucose remained unaffected, 
while meal-induced glucose oxidation may have been impaired at the systemic level by competition with circulating fatty acids in obese mice.

The ability to study exogenous fat oxidation with isotopic sensor extended InCa provided renewed insights to interpret the fasting-and-refeeding challenge as a test of metabolic flexibility. Remarkably, exogenous palmitate was oxidised directly after administration of the meal, a time when fat oxidation was suppressed at the whole body level attributable to increased circulating insulin levels ${ }^{40}$. Likely, intestinal enterocytes were the first site of oxidation of exogenous palmitate directly after consumption of the meal, which is supported by human data showing that orally administered trioleate is oxidised more readily than intravenous trioleate ${ }^{32}$, and that intestinal mucosa can readily oxidise about $17 \%$ of luminal palmitate in rats ${ }^{41}$. In line, fish omega- 3 fatty acids us plant omega- 3 fatty acids were shown to induce fatty acid oxidation rates in the small intestines of mice ${ }^{42}$. Similar to what can be derived from RER data, our findings seem to indicate that oxidation of exogenous fatty acids is not a feature of metabolic flexibility in the early postprandial period. However, we found that exogenous palmitic acid oxidation correlated negatively with body fat mass in the late postprandial and post-absorptive periods (defined in this study as 2-13 h after ingestion of the liquid mixed meal; Fig. 5d). Moreover, the proportion of exogenous palmitic acid oxidised relative to total fatty acid oxidation not only correlated negatively with fat mass (Fig. 5e,f), but also correlated positively with $\triangle$ RER (Fig. 5g,h). Although speculative, it is possible that a blunted exogenous fatty acid oxidation is an unrecognised feature of metabolic inflexibility in response to a mixed meal.

Our observed ambient ${ }^{13} \mathrm{CO}_{2}$ enrichment levels (1.130 atom\%) were not completely consistent with literature values, which are reported to vary between 1.100 to 1.103 atom\% (refs. $3,43,44$ ). This may be due to the ventilation characteristics of the animal room, because the magnitude of the difference in ${ }^{13} \mathrm{CO}_{2}$ enrichment between wheat- and maize-based diets (0.012 atom\%) was close to the measured values of the diets using EA-IRMS (0.008 atom\%). Moreover, the circadian variation in exhaled ${ }^{13} \mathrm{CO}_{2}$ enrichment of mice on non-labelled diets was as anticipated ${ }^{5,45}$. We also observed during the late postprandial and post-absorptive period following the ${ }^{13} \mathrm{C}$-labelled meals, that exogenous glucose oxidation exceeded total glucose. This likely is a direct consequence of the fact that a large part of the RER values fell below 0.704. Below this value, net glucose utilisation is considered to be zero (ref. 22). More precisely, glucose oxidation can continue to take place, with ketogenesis and gluconeogenesis from amino acids contributing to a decrease in RER and effectively leading to an underestimation of glucose oxidation ${ }^{1,22}$. In our study, such processes could be expected as the mice were fasted for $24 \mathrm{~h}$ prior to the food restriction, which was with a high fat (i.e. a relative ketogenic) diet and returned to shortterm negative energy balance already $4 \mathrm{~h}$ after ingesting the single mixed meal. The higher exogenous glucose oxidation compared to total glucose oxidation could also be an indirect consequence of the delayed appearance of the ${ }^{13} \mathrm{C}$ label in ${ }^{13} \mathrm{CO}_{2}$, a known phenomenon due to label retention in the bicarbonate pool in human studies ${ }^{46,47}$ and in agreement with the half-life of $\mathrm{CO}_{2}$ of about $15 \mathrm{~min}$ in mice ${ }^{48}$. In humans, label sequestration in de novo synthesized glucose, glutamine, and glutamate in fatty acid tracer studies likely pertains also to glucose tracers ${ }^{49}$, although this is yet to be investigated in mice. Together with the 
unrealistically high ${ }^{13} \mathrm{C}$ label recoveries we obtained after applying a human dietary acetate recovery factor (dARF) to the situation in mice, our data illustrates the need for a speciesspecific correction factor.

Integrating InCa with ${ }^{13} \mathrm{CO}_{2}$ and ${ }^{12} \mathrm{CO}_{2}$ sensors will improve the interpretation of mouse metabolic studies and provide crucial quantitative data. In addition, it will allow a wide variety of specific substrates to be studied, including metabolic substrates with highly variable or prolonged oxidation kinetics. Substrate-specificity could be further aided by the inclusion of other gas sensors. Moreover, a similar sensor technology would obviate some experimental challenges that remain in metabolic research with stable isotopes in humans, like the necessity to interrupt InCa sessions to obtain separate samples for ${ }^{13} \mathrm{CO}_{2}$ analysis.

In conclusion, the analysis of ${ }^{13} \mathrm{CO}_{2}$ enrichment coupled to conventional InCa is a powerful and targeted tool to quantify the kinetics of exogenous substrate oxidation. We have incorporated ${ }^{13} \mathrm{CO}_{2}$ and ${ }^{12} \mathrm{CO}_{2}$ sensors into a commercial InCa system for use in the mouse, a human-relevant model organism, and demonstrated its value to study fuel use strategies in physiological conditions, non-invasively, and continuously over long experimental periods.

\section{Author Contributions}

J.M.S.F.-C., A.O., J.K., and E.M.v.S. designed the work. J.M.S.F.-C., L.M.S.B., H.J.M.S., and E.M.v.S. acquired the data. J.M.S.F.-C., J.K., and E.M.v.S. analysed and interpreted the data. J.M.S.F.-C. drafted the manuscript and L.M.S.B., A.O., J.K., and E.M.v.S. revised it critically for important intellectual content. All authors critically revised and approved the manuscript.

\section{Acknowledgements}

We thank Remia (Den Dolder, The Netherlands) for their gift of palm olein, Cargill R\&D Centre Europe (Vilvoorde, Belgium) for providing the starches and a free sample of Emultop IP, and Ries de Visser (IsoLife, Wageningen, The Netherlands) for fruitful discussions on tracer studies. We also thank the personnel at the CARUS animal facility for their assistance in mouse care-taking. 


\section{References}

Ferrannini, E. The theoretical bases of indirect calorimetry: A review. Metabolism 37, 287-301 (1988).

Speakman, J. R. Measuring energy metabolism in the mouse - theoretical, practical, and analytical considerations. Front. Physiol. 4, 34 (2013).

Welch, K. C., Jr., Peronnet, F., Hatch, K. A., Voigt, C. C. \& McCue, M. D. Carbon stable-isotope tracking in breath for comparative studies of fuel use. Ann. N. Y. Acad. Sci. 1365, 15-32 (2016).

de Laeter, J. R. et al. Atomic weights of the elements. Review 2000 (IUPAC Technical Report). Pure Appl. Chem. 75, 683-800 (2003).

McCue, M. D. \& Welch, K. C. ${ }^{13}$ C-Breath testing in animals: theory, applications, and future directions. J. Comp. Physiol. B 186, 265-285 (2015).

van den Borne, J. J. et al. Body fat deposition does not originate from carbohydrates in milk-fed calves. J. Nutr. 137, 2234-2241 (2007).

Bruininx, E. et al. Oxidation of dietary stearic, oleic, and linoleic acids in growing pigs follows a biphasic pattern. J. Nutr. 141, 1657-1663 (2011).

McCue, M. D., Voigt, C. C., Jefimow, M. \& Wojciechowski, M. S. Thermal acclimation and nutritional history affect the oxidation of different classes of exogenous nutrients in Siberian hamsters, Phodopus sungorus. J. Exp. Zool. A Ecol. Genet. Physiol. 321, 503-514 (2014).

Isken, F. et al. Impairment of fat oxidation under high- vs. low-glycemic index diet occurs before the development of an obese phenotype. Am. J. Physiol. Endocrinol. Metab. 298, E287-295 (2010).

Friedrich, M., Petzke, K. J., Raederstorff, D., Wolfram, S. \& Klaus, S. Acute effects of epigallocatechin gallate from green tea on oxidation and tissue incorporation of dietary lipids in mice fed a high-fat diet. Int. J. Obes. 36, 735-743 (2012).

Michalski, M. C., Briard, V., Desage, M. \& Geloen, A. The dispersion state of milk fat influences triglyceride metabolism in the rat--a ${ }^{13} \mathrm{CO}_{2}$ breath test study. Eur. J. Nutr. 44, 436-444 (2005).

Butts, C. A. et al. The fate of ${ }^{13} \mathrm{C}$-labelled and non-labelled inulin predisposed to large bowel fermentation in rats. Food. Funct. 7, 1825-1832 (2016).

Fromentin, C. et al. The postprandial use of dietary amino acids as an energy substrate is delayed after the deamination process in rats adapted for 2 weeks to a high protein diet. Amino acids $\mathbf{4 0}$, 1461-1472 (2011).

Ishihara, K. et al. A soybean peptide isolate diet promotes postprandial carbohydrate oxidation and energy expenditure in type II diabetic mice. J. Nutr. 133, 752-757 (2003).

McCue, M. D. \& Pollock, E. D. Measurements of substrate oxidation using ${ }^{13} \mathrm{CO}_{2}$-breath testing reveals shifts in fuel mix during starvation. J. Comp. Physiol. B. 183, 1039-1052 (2013).

Fernández-Calleja, J. M. S. et al. Non-invasive continuous real-time in vivo analysis of microbial hydrogen production shows adaptation to fermentable carbohydrates in mice. Sci. Rep. 8, 15351 (2018).

Hoevenaars, F. P. et al. Effects of dietary history on energy metabolism and physiological parameters in C57BL/6] mice. Exp. Physiol. 98, 1053-1062 (2013).

Reeves, P. G., Nielsen, F. H. \& Fahey, G. C., Jr. AIN-93 purified diets for laboratory rodents: final report of the American Institute of Nutrition ad hoc writing committee on the reformulation of the AIN-76A rodent diet. J. Nutr. 123, 1939-1951 (1993).

Fernandez-Calleja, J. M. S. et al. Direct and long-term metabolic consequences of lowly vs. highlydigestible starch in the early post-weaning diet of mice. Nutrients 10,1788 (2018).

Warner, D., Ferreira, L. M., Breuer, M. J., Dijkstra, J. \& Pellikaan, W. F. Stable isotope labeled n-alkanes to assess digesta passage kinetics through the digestive tract of ruminants. PLoS One $\mathbf{8}$, e75496 (2013). 
Wopereis, S. et al. Multi-parameter comparison of a standardized mixed meal tolerance test in healthy and type 2 diabetic subjects: the PhenFlex challenge. Genes Nutr. 12, 21 (2017).

Peronnet, F. \& Massicotte, D. Table of nonprotein respiratory quotient: an update. Can. J. Sport Sci. 16, 23-29 (1991).

Weir, J. B. d. V. New methods for calculating metabolic rate with special reference to protein metabolism. J. Physiol. 109, 1-9 (1949).

Gold, I. L., Ukhun, M. E. \& Akoh, C. C. Characteristics of eutectic compositions of restructured palm oil olein, palm kernel oil and their mixtures. J. Am. Oil Chem. Soc. 88, 1659-1667 (2011).

Antoun, E. et al. The $\left[1-{ }^{13} \mathrm{C}\right]$ acetate recovery factor to correct tracer-derived dietary fat oxidation is lower in overweight insulin-resistant subjects. E Spen Eur. E J. Clin. Nutr. Metab. 5, e173-e179 (2010).

Bergouignan, A., Schoeller, D. A., Votruba, S., Simon, C. \& Blanc, S. The acetate recovery factor to correct tracer-derived dietary fat oxidation in humans. Am. J. Physiol. Endocrinol. Metab. 294, E645$653(2008)$

Sidossis, L. S., Coggan, A. R., Gastaldelli, A. \& Wolfe, R. R. A new correction factor for use in tracer estimations of plasma fatty-acid oxidation. Am. J. Physiol. Endocrinol. Metab. 269, E649-E656 (1995).

Bessesen, D. H., Bull, S. \& Cornier, M. A. Trafficking of dietary fat and resistance to obesity. Physiol. Behav. 94, 681-688 (2008).

Flatt, J. P., Ravussin, E., Acheson, K. J. \& Jequier, E. Effects of dietary fat on postprandial substrate oxidation and on carbohydrate and fat balances. J. Clin. Invest. 76, 1019-1024 (1985).

Bessesen, D. H., Vensor, S. H. \& Jackman, M. R. Trafficking of dietary oleic, linolenic, and stearic acids in fasted or fed lean rats. Am. J. Physiol. Endocrinol. Metab. 278, E1124-1132 (2000).

Leyton, J., Drury, P. J. \& Crawford, M. A. Differential oxidation of saturated and unsaturated fatty acids in vivo in the rat. Brit. J. Nutr. 57, 383-393 (1987).

Metges, C. C. \& Wolfram, G. Medium- and long-chain triglycerides labeled with ${ }^{13} \mathrm{C}$ : a comparison of oxidation after oral or parenteral administration in humans. J. Nutr. 121, 31-36 (1991).

Binnert, C. et al. Influence of human obesity on the metabolic fate of dietary long- and medium-chain triacylglycerols. Am. J. Clin. Nutr. 67, 595-601 (1998).

Vors, C. et al. Modulating absorption and postprandial handling of dietary fatty acids by structuring fat in the meal: a randomized crossover clinical trial. Am. J. Clin. Nutr. 97, 23-36 (2013).

Galgani, J. E., Moro, C. \& Ravussin, E. Metabolic flexibility and insulin resistance. Am. J. Physiol. Endocrinol. Metab. 295, E1009-1017 (2008).

Duivenvoorde, L. P., van Schothorst, E. M., Swarts, H. J. \& Keijer, J. Assessment of metabolic flexibility of old and adult mice using three noninvasive, indirect calorimetry-based treatments. J. Gerontol. A Biol. Sci. Med. Sci. 70, 282-293 (2015).

Bouwman, L. M. S. et al. No adverse programming by post-weaning dietary fructose of body weight, adiposity, glucose tolerance, or metabolic flexibility. Mol. Nutr. Food Res. 62, 1700315 (2018).

Horakova, O. et al. Preservation of metabolic flexibility in skeletal muscle by a combined use of n-3 PUFA and rosiglitazone in dietary obese mice. PLoS One 7, e43764 (2012).

Asterholm, I. W. \& Scherer, P. E. Enhanced metabolic flexibility associated with elevated adiponectin levels. Am. J. Pathol. 176, 1364-1376 (2010). insulin sensitivity and the metabolic disturbances of diabetes mellitus. Lancet 281, 785-789 (1963). compartmentation and mechanisms of control. J. Clin. Invest. 55, 803-813 (1975). 


\section{CHAPTER 3}

43 Kuc, T. et al. Two decades of regular observations of ${ }^{14} \mathrm{CO}_{2}$ and ${ }^{13} \mathrm{CO}_{2}$ content in atmospheric carbon dioxide in central Europe: long-term changes of regional anthropogenic fossil $\mathrm{CO}_{2}$ emissions. Radiocarbon 49, 807-816 (2016).

44 Keeling, C. D. et al. in A History of Atmospheric $\mathrm{CO}_{2}$ and Its Effects on Plants, Animals, and Ecosystems (eds I. T. Baldwin et al.) 83-113 (Springer, New York, 2005).

45 Wolfe, R. R., Shaw, J. H., Nadel, E. R. \& Wolfe, M. H. Effect of substrate intake and physiological state on background ${ }^{13} \mathrm{CO}_{2}$ enrichment. J. Appl. Physiol. Respir. Environ. Exerc. Physiol. 56, 230-234 (1984).

46 Ravussin, E. et al. Carbohydrate utilization in obese subjects after an oral load of $100 \mathrm{~g}$ naturallylabelled ${ }^{13} \mathrm{C}$ glucose. Br. J. Nutr. 43, 281-288 (1980).

47 Daly, M. E. et al. Acute fuel selection in response to high-sucrose and high-starch meals in healthy men. Am. J. Clin. Nutr. 71, 1516-1524 (2000).

48 Speakman, J. R. \& Thomson, S. C. Validation of the labeled bicarbonate technique for measurement of short-term energy expenditure in the mouse. Z. Ernahrungswiss 36, 273-277 (1997).

49 Schrauwen, P. et al. Validation of the $\left[1,2-{ }^{13} \mathrm{C}\right]$ acetate recovery factor for correction of $\left[\mathrm{U}-{ }^{13} \mathrm{C}\right]$ palmitate oxidation rates in humans. J. Physiol. 513, 215-223 (1998). 


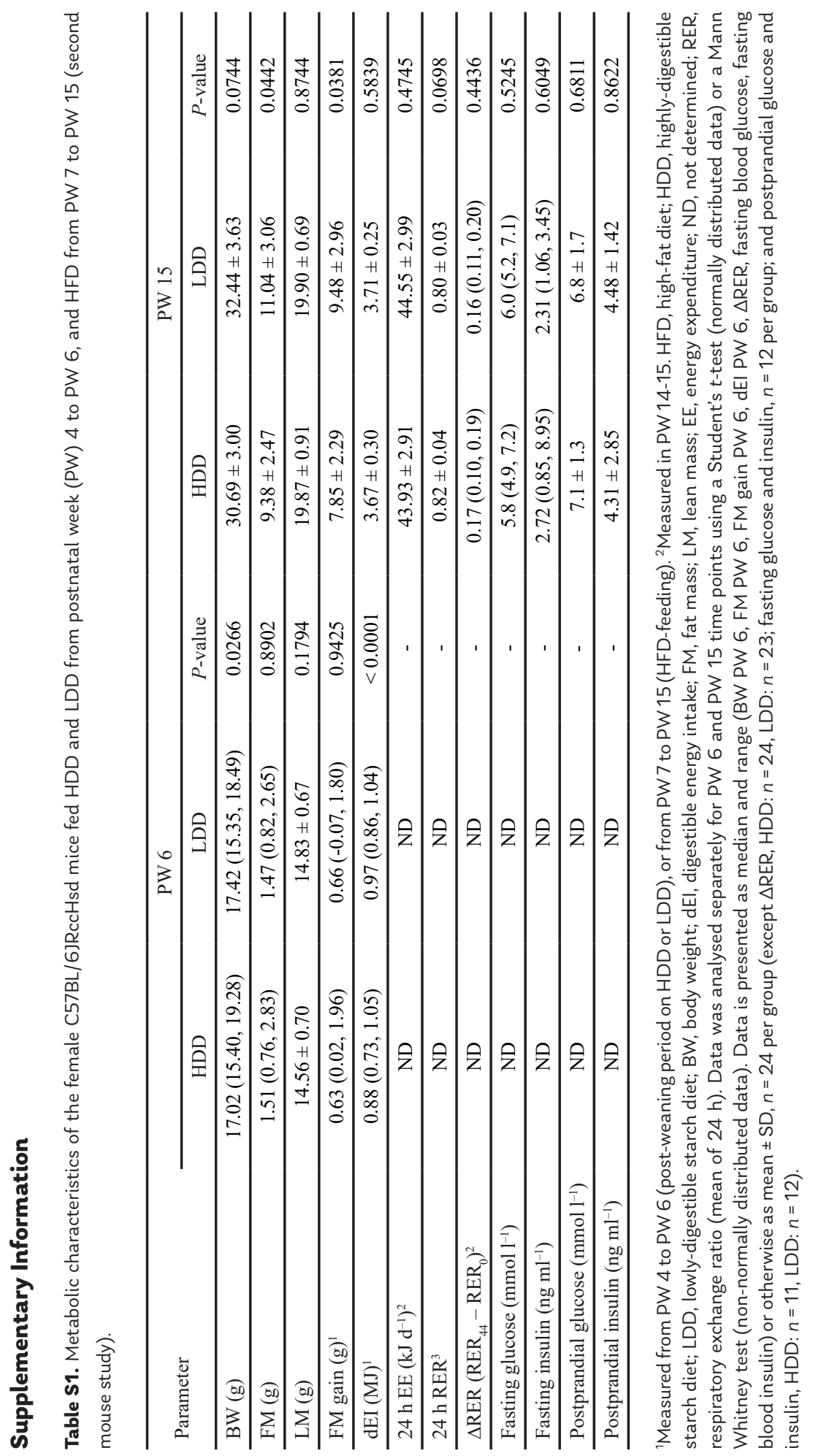


a

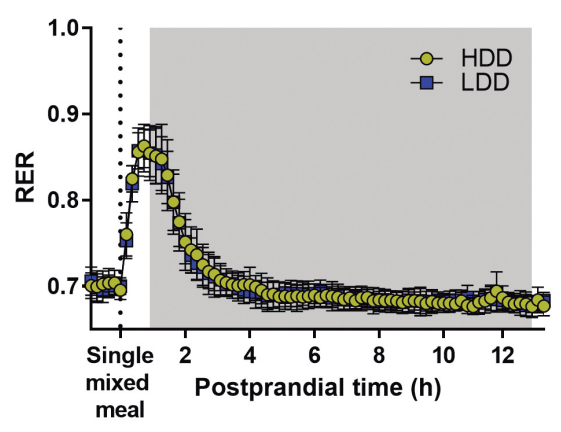

C

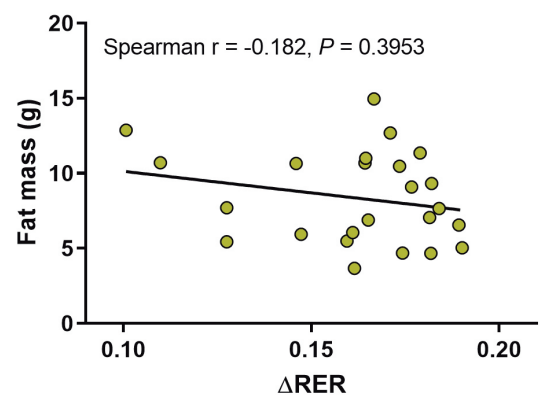

b

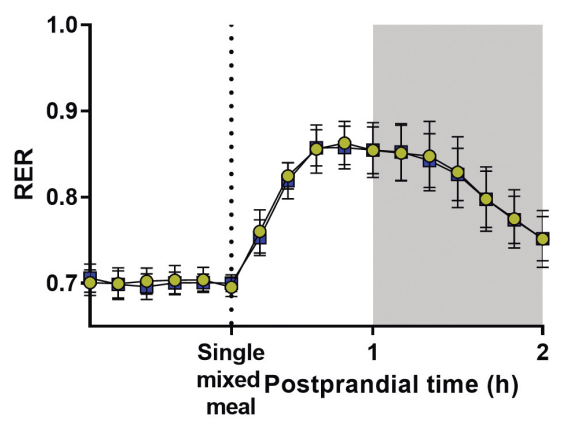

d

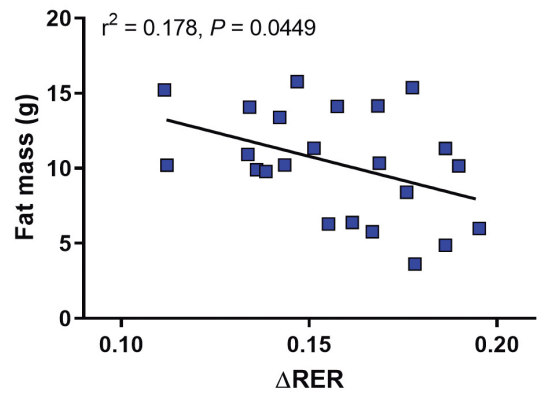

Figure S1. Refeeding metabolic response to a liquid mixed meal and its relation to body fat mass (FM) in mice fed a HFD for nine weeks (second mouse study). (a) Respiratory exchange ratio (RER) was measured in PW 14-15 after gavage of a single liquid mixed meal containing 36 energy\% glucose and 64 energy\% fat in mice fed the HDD ( $n$ $=24)$ or the LDD $(n=23)$ during early life and subsequently fed a HFD for nine weeks. Data was analysed with repeated-measures 2-way analysis of variance to directly test the hypothesis that RER response is influenced by the post-weaning diet. There were no significant effects of the post-weaning diet or its interaction with time. Data is presented as mean \pm SD. Shaded areas represent the dark phase. (b) Data from panel (a), emphasizing the early postprandial $2 \mathrm{~h}$ period. Correlation analysis between $F M$ and the re-feeding response ( $\triangle R E R$ ), measured as the increase from baseline RER to RER at 44 min postprandial, in mice originally fed HDD (c) or LDD (d) during early post-weaning. HDD, highly digestible starch-diet; LDD, lowly digestible starch-diet. 

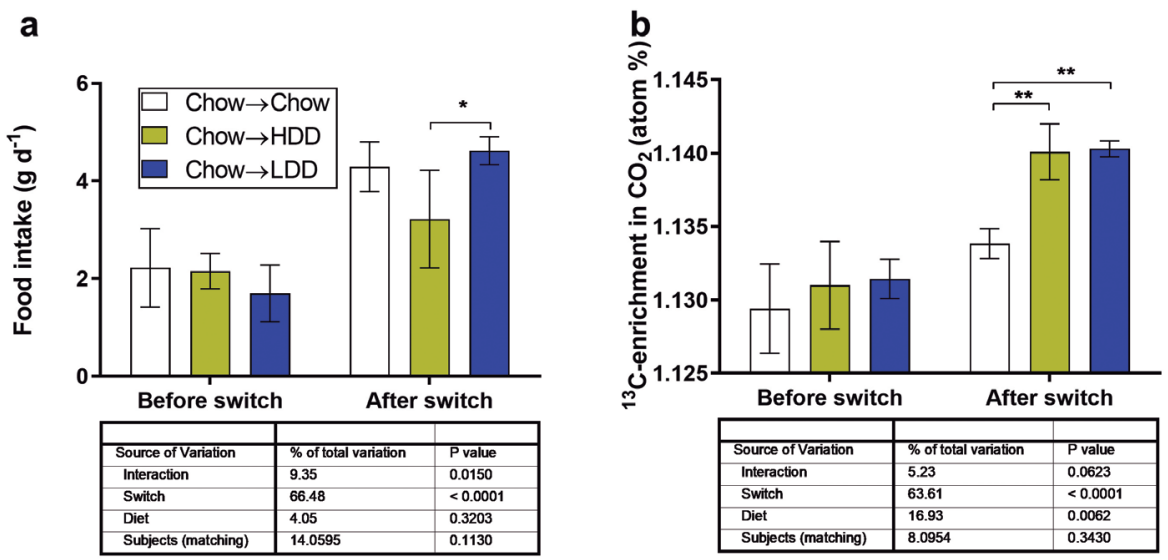

Figure S2. Changes in food intake and ${ }^{13} \mathrm{CO}_{2}$ enrichment in mice fed chow and then switched HDD or LDD, or remaining on chow. Chow-fed mice $(n=12)$ were adapted to indirect calorimetry cages for $2 \mathrm{~d}$, after which mice were allowed a restricted amount $(1.1 \mathrm{~g})$ of chow $1 \mathrm{~h}$ before the onset of the dark phase. At the end of the following light phase, mice were re-fed with a restricted amount $(1.1 \mathrm{~g})$ of chow, or HDD or LDD $(n=4)$. Before the following dark phase mice received ad libitum access to the same diet they were allocated the day before, and measurements continued for $5.5 \mathrm{~d}$ (see Chapter 2 for details). (a) $24 \mathrm{~h}$ cumulative food intake and (b) $24 \mathrm{~h}$ mean ${ }^{13} \mathrm{C}$ enrichment immediately before food restriction and the day after ad libitum re-feeding. Tables show statistical output of repeated-measures 2-way analysis of variance. ${ }^{*} P<0.05,{ }^{* *} P<0.01$, Bonferroni multiple comparisons. Data is presented as mean $\pm S D$. HDD, highly digestible starch-diet; LDD, lowly digestible starch-diet. 



\section{Chapter}

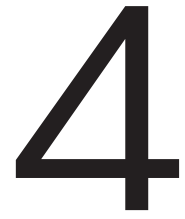

\section{A Lowly Digestible-Starch Diet After Weaning Enhances Exogenous Glucose Oxidation Rate in Female, but Not in Male, Mice}

José M. S. Fernández-Calleja

Lianne M. S. Bouwman

Hans J. M. Swarts

Nils Billecke

Annemarie Oosting

Jaap Keijer

Evert M. van Schothorst 



\section{Abstract}

Starches of low digestibility, compared to highly digestible starches, are associated with improved glucose metabolism in rodents. Most studies have been conducted in males, but available evidence suggests that the metabolic effects of starch digestibility are sexdependent. Here, we test the hypothesis that a lowly digestible starch-diet (LDD) us a highly digestible starch diet (HDD) improves the capacity to oxidise the starch molecule, and that this effect is stronger in females than in males. Female and male mice were fed an LDD or an HDD for 3 weeks directly after weaning. Body weight (BW), body composition (BC), and digestible energy intake ( $\mathrm{dEI})$ were determined weekly. At the end of the intervention period, whole-body energy expenditure (EE), respiratory exchange ratio (RER), hydrogen $\left(\mathrm{H}_{2}\right)$ production, and the oxidation of an oral ${ }^{13} \mathrm{C}$-labelled starch bolus were measured by extended indirect calorimetry. Additionally, pancreatic amylase activity and total ${ }^{13} \mathrm{C}$ hepatic enrichment were determined in females sacrificed immediately before and $4 \mathrm{~h}$ after administration of the starch bolus. For both sexes, BW, BC, and basal EE and RER were not affected by the type of starch, but $\mathrm{dEI}$ and $\mathrm{H}_{2}$ production were increased by the LDD. Only in females, total carbohydrate oxidation and starch-derived glucose oxidation in response to the starch bolus were higher in LDD us HDD mice, and this was not accompanied by differences in amylase activity or partitioning of the ${ }^{13} \mathrm{C}$ label toward the liver. These results show that starch digestibility impacts glucose metabolism differently in females compared to males.

Keywords: Indirect calorimetry, C57BL mice, glucose oxidation, ${ }^{13} \mathrm{C}$-starch, amylose, amylopectin, glycaemic index, amylase. 


\section{Introduction}

The digestibility of dietary starch can influence metabolic health. This has been extensively studied in rodents, where lowly digestible starches has led to lower body weight (BW) and adiposity and normal glucose homeostasis compared to highly digestible starches ${ }^{1}$. In humans, lowly digestible starches have been shown to improve insulin sensitivity and other metabolic endpoints ${ }^{2-5}$, although more evidence is needed to establish how this type of starches should be included in an individual's diet to bring about their beneficial effects. For instance, while a lower glycaemic response is achieved by replacing glycaemic carbohydrates with lowly digestible starches, it is not clear whether this is also the case when available carbohydrates remain constant ${ }^{6}$, or whether factors like differences in gut microbiota composition could change how lowly digestible starches affect specific subgroups of people ${ }^{7}$ Robertson, 2012 \#1011;Peterson, 2018 \#1414;Peterson, $2018 \# 1414$ \}. A previous study showed higher postprandial exogenous and total glucose oxidation in women compared to men. which was associated with intrinsic differences in insulin sensitivity and body composition ${ }^{8}$. Whether or not the metabolic impact of starches of different digestibility also depends on sex is currently unknown.

A recent meta-analysis of rodent studies showed that consuming lowly digestible starches results in similar BW and fat mass (FM) compared to highly digestible starch feeding in females ${ }^{1}$. In contrast, we have observed that feeding a lowly digestible starch diet (LDD) us a highly digestible starch diet (HDD) for three weeks directly after weaning resulted in smaller adipocytes and less crown-like structures in gonadal white adipose tissue (WAT) despite similar BW and body composition (BC) ${ }^{9}$. After exposure to a high-fat diet in adulthood, these females developed a higher metabolic flexibility to a starch-containing meal, which was not observed in male mice ${ }^{9}$. Some effects of starch digestibility on metabolic health have been shown to precede alterations in BW or adiposity in mice. Adult males on a highly digestible starch for three weeks showed lower exogenous fat oxidation compared to those fed lowly digestible starch, prior to developing an obese phenotype 10. This raises the question whether exogenous substrate oxidation is also influenced by exposure to starches of different digestibility in females of similar BW and BC.

In this study, we tested the hypothesis that exposure to an LDD compared to an HDD during the immediate post-weaning period leads to a better capacity to oxidise dietary carbohydrates, specifically the starch molecule, in female but not in male mice. Additionally, we explore whether small intestinal amylase levels or the partitioning of starch-derived glucose to the liver compartment could explain any potential sex differences in starch oxidation.

\section{Materials and Methods}

Mouse experiment

All mice (C57BL/6]RccHsd, Envigo, Horst, The Netherlands) were individually housed in Makrolon II cages with wood chips and wood shavings, at $23 \pm 1{ }^{\circ} \mathrm{C}, 50 \pm 5 \%$ humidity, on a 
$12 \mathrm{~h}$ light/dark cycle (6.00 AM lights on). Unless otherwise indicated, mice had ad libitum access to food and water.

Adult mice (9-23 weeks old) on a chow diet (Teklad Global Diet 2920, Envigo) were time-mated and their offspring cross-fostered within 24-48 h postnatally to produce standardized litters of 6 pups and a sex ratio of 3:3 or 4:2. At the end of postnatal week (PW) 3, male and female offspring were weaned and individually housed and stratified by BW to receive either an LDD (Research Diet Services, Wijk bij Duurstede, The Netherlands; $n=15$ females, $n=8$ males) or an HDD (Research Diet Services; $n=15$ females, $n=9$ males) for the rest of the study. These two diets only differed in the type of starch included. The experimenter was not blinded to the experimental diets. Welfare was monitored daily by visual inspection. BW, body composition (BC; EchoMRI 100V, EchoMedical Systems, Houston, Texas, USA), and food intake (FI) were determined weekly. At the end of PW 6 (for females) or during PW 7 (for males), mice were re-stratified by BW and assigned to be sacrificed in either the fasting state or after ingestion of a ${ }^{13} \mathrm{C}$-labelled starch bolus (described below). Additionally, a subgroup of the mice assigned the ${ }^{13} \mathrm{C}$ starch bolus was transferred to an extended indirect calorimetry ( $\mathrm{InCa}$ ) system for measurements of wholebody metabolism and total and starch-derived glucose oxidation (described below). The study was approved by the CCD/IvD 2017.W-0024.002 and performed in accordance with European Union (EU) directive 2010/63/EU.

\section{Diet composition}

The chow diet for breeding and lactating dams consisted of 24, 60, and 16 energy\% protein, carbohydrate, and fat, respectively. The semi-purified LDD and HDD contained 20,55 , and 25 energy\% protein, carbohydrate, and fat, respectively, and were based on the BIOCLAIMS standard diet ${ }^{11}$. The starches in the LDD and HDD (569 $\mathrm{g} \mathrm{kg}^{-1}$ diet; Cargill, Sas van Gent, The Netherlands) were incorporated by Research Diet Services. The exact composition and digestible energy density of the diets are shown in Table 1.

Preparation and administration of the ${ }^{13} \mathrm{C}$-labelled starch bolus

A $20 \mathrm{mg} \mathrm{ml}^{-1}$ mixture of uniformly ${ }^{13} \mathrm{C}$-labelled potato starch $\left(98.2\right.$ atom $\%{ }^{13} \mathrm{C}$, > 98\% dry $\mathrm{w} / \mathrm{w}$ glucan chemical purity; IsoLife, Wageningen, The Netherlands) in phosphate buffered saline (PBS) was heated to $90{ }^{\circ} \mathrm{C}$ for $15 \mathrm{~min}$ and added to a $120 \mathrm{mg} \mathrm{ml}^{-1}$ suspension of non-labelled amylopectin maize starch ( $C^{*}$ Gel 04201, Cargill) in PBS, to achieve a total starch concentration of $80 \mathrm{mg} \mathrm{ml}^{-1}$. Preparations were made fresh on the day of use and kept under constant stirring before administration to the mice.

On the day prior to the administration of the ${ }^{13} \mathrm{C}$ starch bolus, mice (including the subgroup studied in $\mathrm{InCa}$ ) were food-restricted approximately $2 \mathrm{~h}$ before the dark phase by receiving 1.21 (SD 0.02) g of their corresponding post-weaning diet (LDD or HDD). The day after, approximately $2 \mathrm{~h}$ into the light phase (LP) when the mice were fasted, each mouse was administered $0.5 \mathrm{ml}$ of the ${ }^{13} \mathrm{C}$ starch preparation by oral gavage, representing a dose of $40 \mathrm{mg}$ of total starch per mouse $(0.6 \mathrm{~kJ})$. 
Table 1. Composition of the experimental diets.

\begin{tabular}{|c|c|c|}
\hline & HDD & LDD \\
\hline Casein $\left(\mathrm{g} \mathrm{kg}^{-1}\right)$ & 212.2 & 212.0 \\
\hline L-Cysteine $\left(\mathrm{g} \mathrm{kg}^{-1}\right)$ & 3.0 & 3.0 \\
\hline Amylose mix (AmyloGel 03003) $\left(\mathrm{g} \mathrm{kg}^{-1}\right) \dagger$ & 0.0 & 568.6 \\
\hline Amylopectin $\left(\mathrm{C}^{*} \mathrm{Gel}\right.$ 04201) $\left(\mathrm{g} \mathrm{kg}^{-1}\right) \ddagger$ & 568.6 & 0.0 \\
\hline Coconut oil $\left(\mathrm{g} \mathrm{kg}^{-1}\right)$ & 21.4 & 21.4 \\
\hline Sunflower oil $\left(\mathrm{g} \mathrm{kg}^{-1}\right)$ & 83.1 & 83.1 \\
\hline Flaxseed oil $\left(\mathrm{g} \mathrm{kg}^{-1}\right)$ & 14.2 & 14.2 \\
\hline Cholesterol $\left(\mathrm{g} \mathrm{kg}^{-1}\right)$ & 0.03 & 0.03 \\
\hline Cellulose $\left(\mathrm{g} \mathrm{kg}^{-1}\right)$ & 50.0 & 50.0 \\
\hline Mineral mix (AIN-93G-MX) $\left(\mathrm{g} \mathrm{kg}^{-1}\right)$ & 35.0 & 35.0 \\
\hline Vitamin mix (AIN-93-VX) $\left(\mathrm{g} \mathrm{kg}^{-1}\right)$ & 10.0 & 10.0 \\
\hline Choline bitartrate $\left(\mathrm{g} \mathrm{kg}^{-1}\right)$ & 2.5 & 2.5 \\
\hline Calculated energy density $\left(\mathrm{kJ} \mathrm{g}^{-1}\right) \S$ & 17.9 & 17.9 \\
\hline Gross energy density $\left(\mathrm{kJ} \mathrm{g}^{-1}\right) \|$ & 18.9 & 19.5 \\
\hline Digestible energy density $\left(\mathrm{kJ} \mathrm{g}^{-1}\right)$ & 17.6 & 17.0 \\
\hline Protein (energy\%) & 20.1 & 20.1 \\
\hline Carbohydrate (energy\%) & 54.9 & 54.9 \\
\hline Fat (energy\%) & 25.0 & 25.0 \\
\hline
\end{tabular}

HDD, highly digestible-starch diet; LDD, lowly digestible-starch diet. $† 60 \%$ amylose, $40 \%$ amylopectin (Cargill). ₹100\% amylopectin (Cargill). § Calculated based on Atwater's nutritional values. II Determined by bomb calorimetry. 9 Determined by bomb calorimetry and faecal output in an independent experiment ${ }^{12}$.

Extended indirect calorimetry $(\ln C a)$

Animals were acclimatised to the extended InCa system (PhenoMaster, TSE Systems, Bad Homburg, Germany) for approximately $36 \mathrm{~h}$. The following $24 \mathrm{~h}$ period was used for measurements of $\mathrm{O}_{2}$ consumption and $\mathrm{CO}_{2}$ production from which daily energy expenditure (EE) and respiratory exchange ratio (RER) were calculated. Locomotor activity, and food and water intake were also measured. Recently, we incorporated hydrogen $\left(\mathrm{H}_{2}\right)$ and methane $\left(\mathrm{CH}_{4}\right)$ sensors for gut microbiota activity measurements in the InCa system ${ }^{12}$. After basal measurements, mice received a limited amount of food ( 1.2 g) and were then administered the ${ }^{13} \mathrm{C}$ starch bolus in the fasted state, as described above, and $\operatorname{lnCa}$ measurements continued for the next $24 \mathrm{~h}$. For logistical reasons, gas sampling frequency was set at 20 min for males and 11 min for females. LDD and HDD animals were equally represented in each InCa measurement. Bedding was reduced to about $200 \mathrm{ml}$ during InCa measurements to facilitate detection of voluntary locomotion by infrared beam breaks in the horizontal plane. BW and BC were determined directly before and after InCa measurement. The technological extensions of the InCa system and other operational settings and procedures have been described previously ${ }^{12,13}$. 
Total glucose oxidation (TGO) rates were calculated from $\mathrm{VO}_{2}$ and $\mathrm{VCO}_{2}$, based on Péronnet's table of non-protein $\mathrm{RER}^{14}$. Starch-derived glucose oxidation (SGO) rates were calculated using the following two formulas:

$$
\begin{gathered}
a t \%{ }^{13} \mathrm{CO}_{2}=\frac{{ }^{13} \mathrm{CO}_{2}}{{ }^{13} \mathrm{CO}_{2}+{ }^{12} \mathrm{CO}_{2}} \times 100 \\
S G O\left(\mathrm{mg} \mathrm{min}^{-1}\right)=\frac{a t{ }^{13} \mathrm{CO}_{2}(t)-a t{ }^{13} \mathrm{CO}_{2}(t 0)}{\left.a t{ }^{13} C_{S}-a t \%^{13} \mathrm{CO}_{2}(+)\right)} \times \frac{V C O_{2}(t)}{22.29 \times 6}
\end{gathered}
$$

The at $\%{ }^{13} \mathrm{CO}_{2}$ (formulas 1 and 2 ) is the ${ }^{13} \mathrm{C}$ enrichment in expired $\mathrm{CO}_{2}$ in atom\% calculated from ${ }^{13} \mathrm{CO}_{2}$ and ${ }^{12} \mathrm{CO} 2$ gas concentrations (delta ppm). In formula 2 (based on ${ }^{8}$ ), the time $t_{0}$ represents the baseline measurement over approximately $1 \mathrm{~h}$ before administration of the ${ }^{13} \mathrm{C}$ starch bolus, and $\mathrm{t}$ represents any subsequent time point. The calculated total ${ }^{13} \mathrm{C}$ enrichment in the starch bolus $\left({ }^{13} \mathrm{C}\right.$-labelled plus non-labelled starch) is represented by at $\%{ }^{13} \mathrm{C}_{\mathrm{s}}$ and has a value of approximately 8.4 atom $\%$, according to the declared isotopic and dry chemical purity of the ${ }^{13} \mathrm{C}$-labelled starch, and assuming a ${ }^{13} \mathrm{C}$ enrichment of 1.1 atom $\%$ of the non-labelled maize starch based on measured values of maize-derived fructose ${ }^{15}$. $\mathrm{VCO}_{2}$ is the production rate of total $\mathrm{CO}_{2}$ obtained using the summed concentrations of ${ }^{13} \mathrm{CO}_{2}$ and ${ }^{12} \mathrm{CO}_{2}$ multiplied by the constant air flow. The molecular weight of glucose is $180.16 \mathrm{~g} \mathrm{~mol}^{-1}$, the volume occupied by $1 \mathrm{~mol}$ of $\mathrm{CO}_{2}$ in STPD is $22.29 \mathrm{l}$, and 6 carbons per mol of starch-derived glucose were ${ }^{13} \mathrm{C}$-labelled. To express SGO rates as percentage of the dose of starch-derived glucose administered, the declared moisture content in the ${ }^{13} \mathrm{C}$-labelled and non-labelled starches and the release of $1.1 \mathrm{~g}$ of glucose per $1 \mathrm{~g}$ of dry starch $^{16}$ were taken into account.

\section{Dissection}

Mice were food-restricted by receiving about $1.2 \mathrm{~g}$ of their corresponding post-weaning diet (LDD or HDD) $2 \mathrm{~h}$ before the dark phase, as described above. On the following day, mice were decapitated either in the fasted state ( $3 \mathrm{~h}$ into the LP), or exactly $4 \mathrm{~h}$ after administration of $\mathrm{a}^{13} \mathrm{C}$ starch bolus ( $5 \mathrm{~h}$ into the LP). No anaesthesia was used, to prevent changes in glucose levels. Glycaemia was determined in whole blood directly after sacrifice using a Freestyle glucose meter (Abbott Diabetes Care, Hoofddorp, The Netherlands). The liver was excised, weighed, separated into lobes, and snap-frozen in liquid $\mathrm{N}_{2}$. The luminal content of the small intestine was gently pressed out, weighed, and snap-frozen. Samples were then stored at $-80^{\circ} \mathrm{C}$.

\section{Amylase activity}

Amylase activity in small intestinal contents was measured with a colorimetric method (Amylase Activity Assay Kit, MAK009, Sigma-Aldrich, Zwijndrecht, The Netherlands) according to the manufacturer's instructions. The intra-assay and inter-assay CVs were 2.2 and $14.8 \%$, respectively. 
Elemental analysis isotope ratio mass spectrometry (EA-IRMS)

We focussed on the partitioning of ${ }^{13} \mathrm{C}$ starch into liver because this organ extracts about $30 \%$ of an enteral glucose load, particularly in fasting conditions ${ }^{17,18}$. Liver total ${ }^{13} \mathrm{C}$ enrichment was measured by EA-IRMS as previously published ${ }^{19}$. Briefly, a sample of the right lobe was freeze-dried and combusted at $1020^{\circ} \mathrm{C}$ in the presence of oxygen to convert carbon into $\mathrm{CO}_{2}$, followed by separation for measurement of the ${ }^{13} \mathrm{C} /{ }^{12} \mathrm{C}$ ratio by EA-IRMS. Results were expressed as delta over baseline (DOB) atom\%, calculated from the algebraic difference between atom $\%{ }^{13} \mathrm{C}$ from individual mice that received $\mathrm{a}^{13} \mathrm{C}$ starch bolus and the mean atom $\%{ }^{13} \mathrm{C}$ of mice on LDD or HDD that did not receive the ${ }^{13} \mathrm{C}$ starch bolus and were sacrificed in the fasted state. Label recovery in the liver compartment was calculated from the dry weight of whole liver and the total $C$ and ${ }^{13} \mathrm{C}$ content in the samples.

\section{Statistical analysis}

Sample size $(n=6)$ was calculated to detect a difference of $0.0466 \mathrm{mg} \mathrm{min}^{-1}$ in maximal exogenous glucose oxidation with $80 \%$ statistical power at a two-sided significance level of 0.05 (G*Power v. 3.1.9.3) $)^{20}$, based on preliminary data with obese and lean mice in our extended InCa system. A single pup was considered one experimental unit. One HDD male mouse was excluded from all data analyses due to incisor malocclusion detected at the end of the study. Normal distribution of the data was tested with the D'Agostino and Pearson omnibus test; non-normally distributed data were log-transformed and retested for normality. When $n \leq 6$, a normal distribution was assumed. Equality of variances was tested with an F-test. Differences between LDD us HDD mice in single outcomes at the end of the study (e.g. BW) were determined separately per sex with a two-tailed unpaired Student's t-test (normally-distributed data) with Welch's correction when variances were significantly different, or with a two-tailed Mann-Whitney test (non-normally distributed data). Time course data (e.g. SGO) was analysed separately per sex by a two-tailed unpaired Student's $t$-test on the incremental area under the curve (iAUC), taking as baseline the average RER over approximately $1 \mathrm{~h}$ before administration of the ${ }^{13} \mathrm{C}$ starch bolus. To locate specific time points showing statistically significant differences, time course data was analysed by repeated-measures (RM) 2-way ANOVA and, when the interaction between time and post-weaning diet was significant, followed by Bonferroni's post hoc test for multiple comparisons. Sphericity was assumed. Two RER values that were not recorded due to opening of the cage were replaced by the average of the previous and next recordings. To compare the cumulative SGO in males us females on LDD or HDD, and to compare parameters measured in LDD us HDD females in the fasted state or $4 \mathrm{~h}$ after receiving the ${ }^{13} \mathrm{C}$ starch bolus, a 2-way ANOVA was used. Statistical analyses, iAUC calculations, and data visualisation were performed in Prism v.5.04 (GraphPad, San Diego, California, USA). Statistical significance was set at $P<0.05$. 


\section{Results}

\section{General metabolic phenotype}

Both in males and females, BW, FM, lean mass, and fasting blood glucose concentrations were not different after three weeks of consuming the post-weaning LDD us HDD (Table 2). Similarly, $24 \mathrm{~h}$ whole-body energy expenditure, respiratory exchange ratio (RER), and locomotor activity were not different in LDD us HDD mice (data not shown). However, cumulative digestible energy intake over the complete post-weaning intervention was about 13\% higher in LDD compared to HDD mice (Table 2), and cumulative hydrogen $\left(\mathrm{H}_{2}\right)$ production over $24 \mathrm{~h}$ was approximately 2.9-fold higher in LDD us HDD mice (Figure 1). Methane $\left(\mathrm{CH}_{4}\right)$ production was undetectable.

Table 2. Body weight, body composition, and cumulative energy intake at the end of the post-weaning nutritional intervention.

\begin{tabular}{|c|c|c|c|c|c|c|c|c|}
\hline & \multicolumn{4}{|c|}{ Males } & \multicolumn{4}{|c|}{ Females } \\
\hline & \multicolumn{2}{|c|}{ HDD } & \multicolumn{2}{|c|}{ LDD } & \multicolumn{2}{|c|}{ HDD } & \multicolumn{2}{|c|}{ LDD } \\
\hline & $\begin{array}{l}\text { Mean or } \\
\text { median }\end{array}$ & $\begin{array}{l}\mathrm{SD} \text { or } \\
\text { range }\end{array}$ & $\begin{array}{l}\text { Mean or } \\
\text { median }\end{array}$ & $\begin{array}{l}\mathrm{SD} \text { or } \\
\text { range }\end{array}$ & $\begin{array}{l}\text { Mean or } \\
\text { median }\end{array}$ & $\begin{array}{l}\mathrm{SD} \text { or } \\
\text { range }\end{array}$ & $\begin{array}{l}\text { Mean or } \\
\text { median }\end{array}$ & $\begin{array}{l}\mathrm{SD} \text { or } \\
\text { range }\end{array}$ \\
\hline Body weight (g) & 21.23 & 1.13 & 21.53 & 2.08 & 16.60 & $\begin{array}{l}14.06 \\
18.54\end{array}$ & 16.72 & $\begin{array}{l}14.01 \\
17.55\end{array}$ \\
\hline Fat mass (g) & 2.14 & 0.43 & 1.74 & 0.52 & 1.69 & 0.60 & 1.43 & 0.33 \\
\hline Lean mass (g) & 18.18 & 0.96 & 18.81 & 1.49 & 14.28 & 1.06 & 14.17 & 0.88 \\
\hline $\begin{array}{c}\text { Fasting blood } \\
\text { glucose }\left(\mathrm{mmol} \mathrm{l}^{-1}\right)\end{array}$ & 4.9 & 0.6 & 5.4 & 0.5 & 4.4 & 0.8 & 4.7 & 0.8 \\
\hline $\begin{array}{l}\text { Digestible energy } \\
\text { intake }(\mathrm{MJ}) \dagger\end{array}$ & 1.15 & $\begin{array}{l}1.12 \\
1.26\end{array}$ & $1.30 * * *$ & $\begin{array}{l}1.20 \\
1.46\end{array}$ & 0.88 & $\begin{array}{l}0.61 \\
0.93\end{array}$ & $0.99 * * * *$ & $\begin{array}{l}0.95 \\
1.06\end{array}$ \\
\hline
\end{tabular}

HDD, highly digestible-starch diet; LDD, lowly digestible-starch diet. † Cumulative digestible energy intake from introduction of post-weaning diet until the end of the study (not including the starch bolus). Data is presented as mean and SD, except for body weight (females) and energy intake (both sexes), where median and range are shown. Males: $n=8-9$, females: $n=9-15$. ${ }^{* *} P<0.001,{ }^{* * *} P<0.0001$.

Food restriction and substrate switching in response to the ${ }^{13} \mathrm{C}$ starch bolus

For both sexes, LDD mice showed a lower RER compared to HDD mice from the time of food restriction up until the moment before administration of the ${ }^{13} \mathrm{C}$ starch bolus (Figure S1). Fasted ( $17 \mathrm{~h}$ after food restriction) LDD males had a higher RER (0.725 (SD 0.015)) compared to HDD males (0.697 (SD 0.009); $P<0.01)$. In contrast, fasting RER was similar between LDD and HDD females (0.678 (SD 0.016) and 0.677 (SD 0.024) respectively). 
a

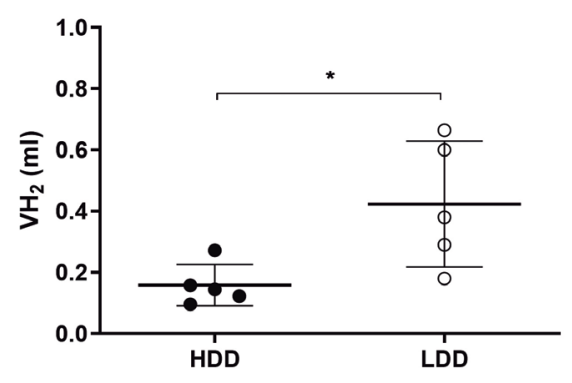

b

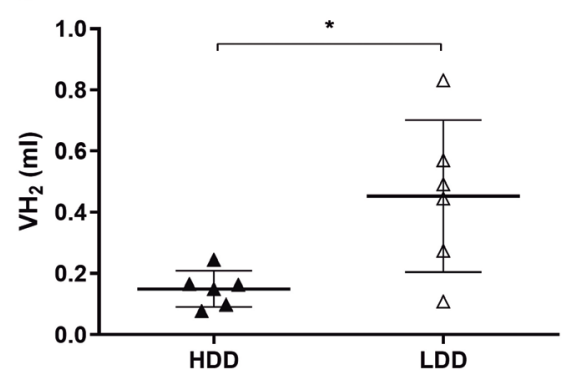

Figure 1. Cumulative $24 \mathrm{~h} \mathrm{H}_{2}$ production on HDD or LDD. (a) Males ( $n=5$; PW 4), (b) females ( $n=6$; PW 3). Data is presented as mean and SD. Student's t-test, ${ }^{*} P<0.05$. HDD, highly digestible-starch diet; LDD, lowly digestible-starch diet; PW, post-weaning week.

In response to administration of the ${ }^{13} \mathrm{C}$ starch bolus, LDD and HDD males showed a similar increase in RER (Figure 2a,b), but RER in LDD males remained higher throughout the $4 \mathrm{~h}$ postprandial period (Figure $2 \mathrm{c}$ ). LDD females had an initially higher increase in RER compared to HDD females (Figure $2 \mathrm{~d}$,e), while both groups maintained a generally similar RER throughout the starch bolus challenge (Figure $2 \mathrm{f}$ ).

Starch-derived and total glucose oxidation in response to the ${ }^{13} \mathrm{C}$ starch bolus

Analysis of ${ }^{13} \mathrm{C}$ enrichment in expired $\mathrm{CO}_{2}$, as a qualitative indication of the oxidation of exogenous ${ }^{13} \mathrm{C}$ starch, showed a trend for a diet $\times$ time interaction in males $(P=0.095)$, and a main effect of the post-weaning diet $(P<0.05$; Figure $\mathrm{S} 2 \mathrm{a})$. Comparing rates of starchderived glucose oxidation, calculated from ${ }^{13} \mathrm{CO}_{2}$ and total $\mathrm{CO}_{2}$ production values, showed no diet $\times$ time interaction in males, with a trend towards an effect of the post-weaning diet with seemingly higher values in LDD males $(P=0.083$; Figure $3 a)$. In addition, energy expenditure upon the administration of the starch bolus was initially higher in HDD males (post-weaning diet $\times$ time interaction, $P=0.045$; Figure S3a). Consistent with the RER response (Figure $2 \mathrm{a}$ ), total glucose oxidation (thus including exogenous starch-derived and endogenous glucose) showed no diet $\times$ time interaction, but showed a significant main effect of the post-weaning diet with higher values in LDD males $(P<0.01$; Figure $3 b)$. Thus, although LDD males exhibited generally higher glucose oxidation rates, the exogenous glucose oxidation response with time was similar in LDD and HDD males.

In contrast to males, the increase in ${ }^{13} \mathrm{CO}_{2}$ enrichment was greater in LDD compared to HDD females (diet $\times$ time interaction, $P<0.0001$; Figure $S 2 b$ ). Similarly, oxidation rates of exogenous starch-derived glucose were also influenced by the post-weaning diet (diet $\times$ time interaction, $P<0.05$ ), being significantly higher in LDD females at 33 and 44 min upon administration of the ${ }^{13} \mathrm{C}$ starch bolus (Figure $3 \mathrm{~d}$ ). Energy expenditure after consumption of the starch bolus was not affected by the post-weaning diet (Figure S3b). 
CHAPTER 4
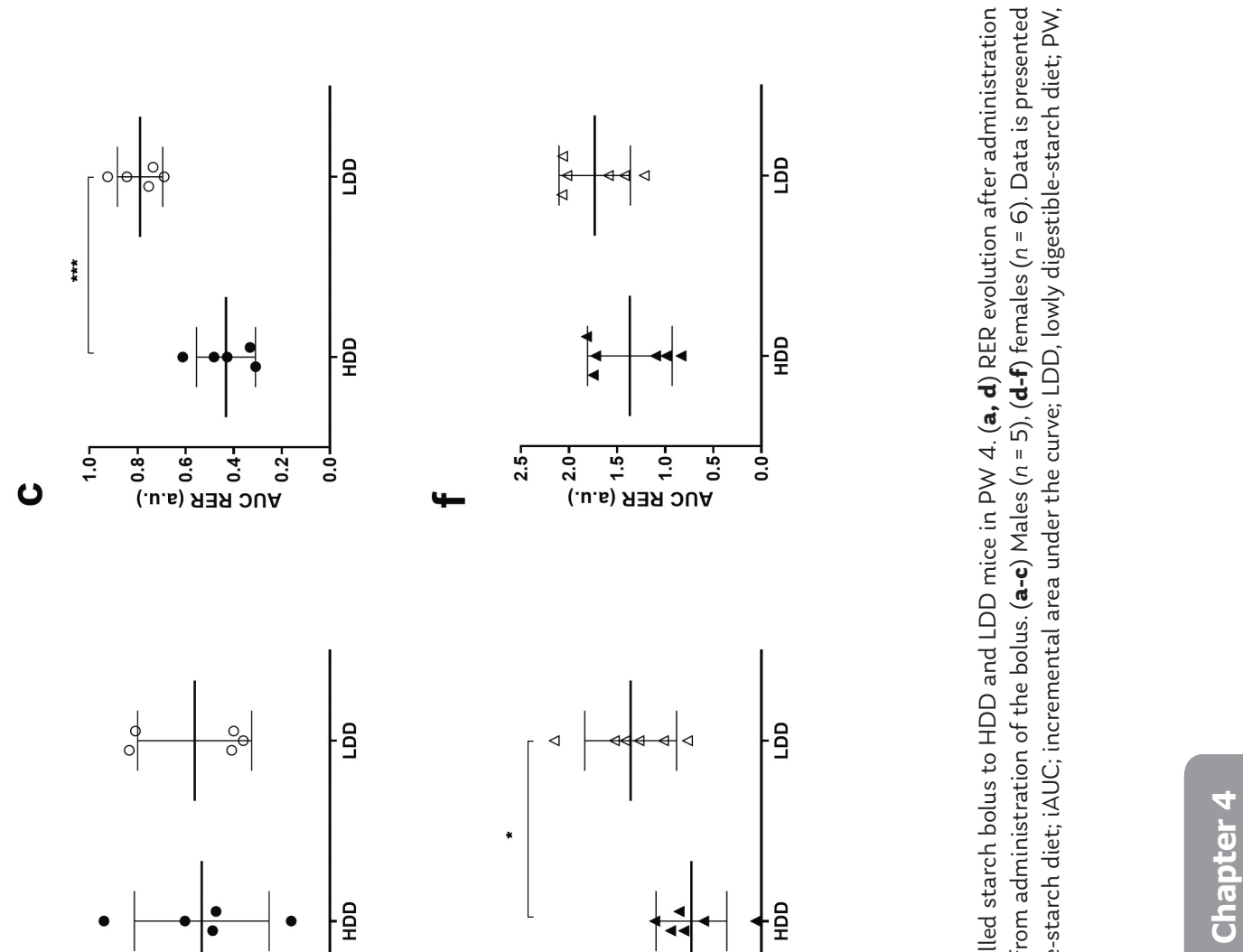

2
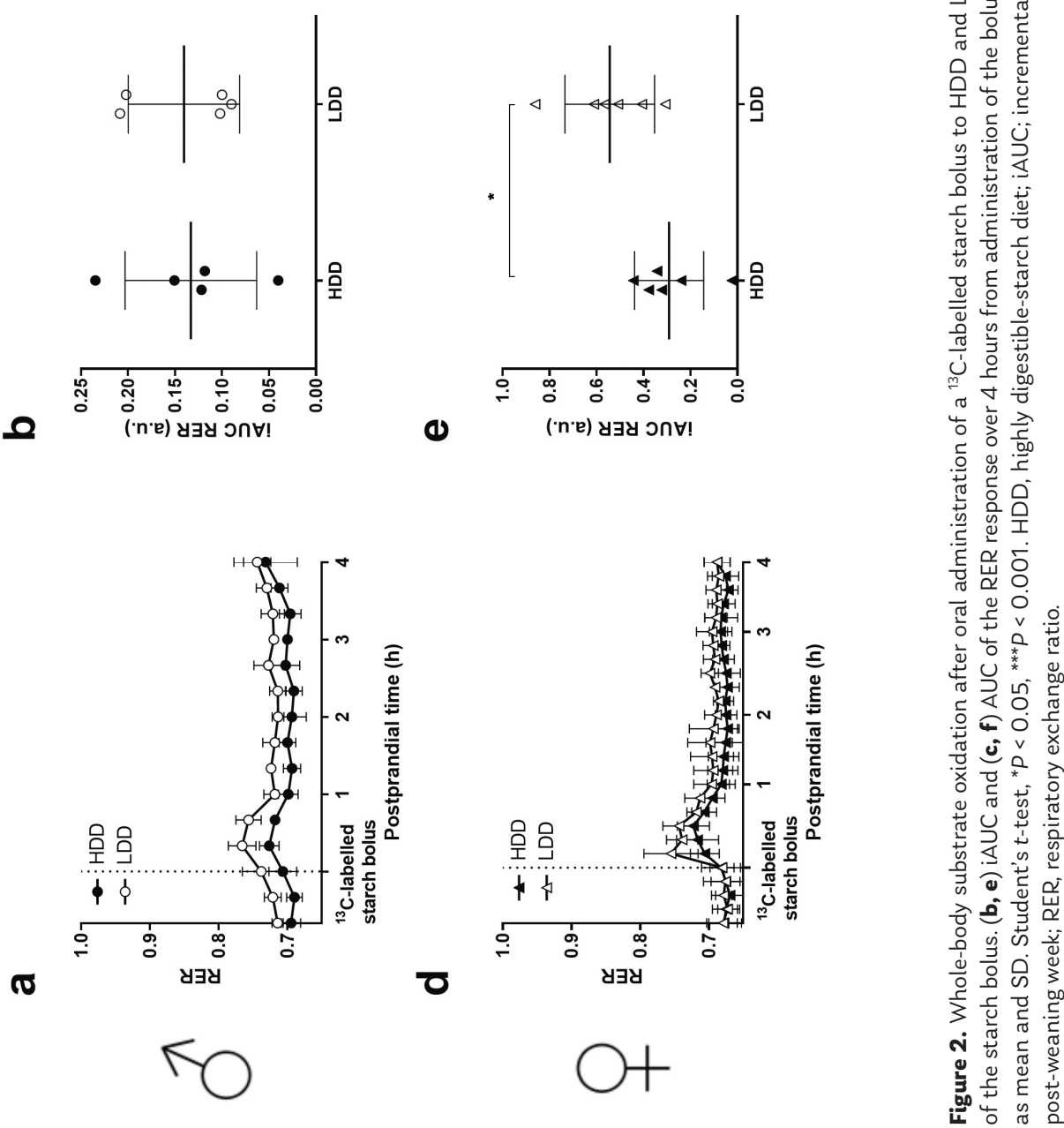

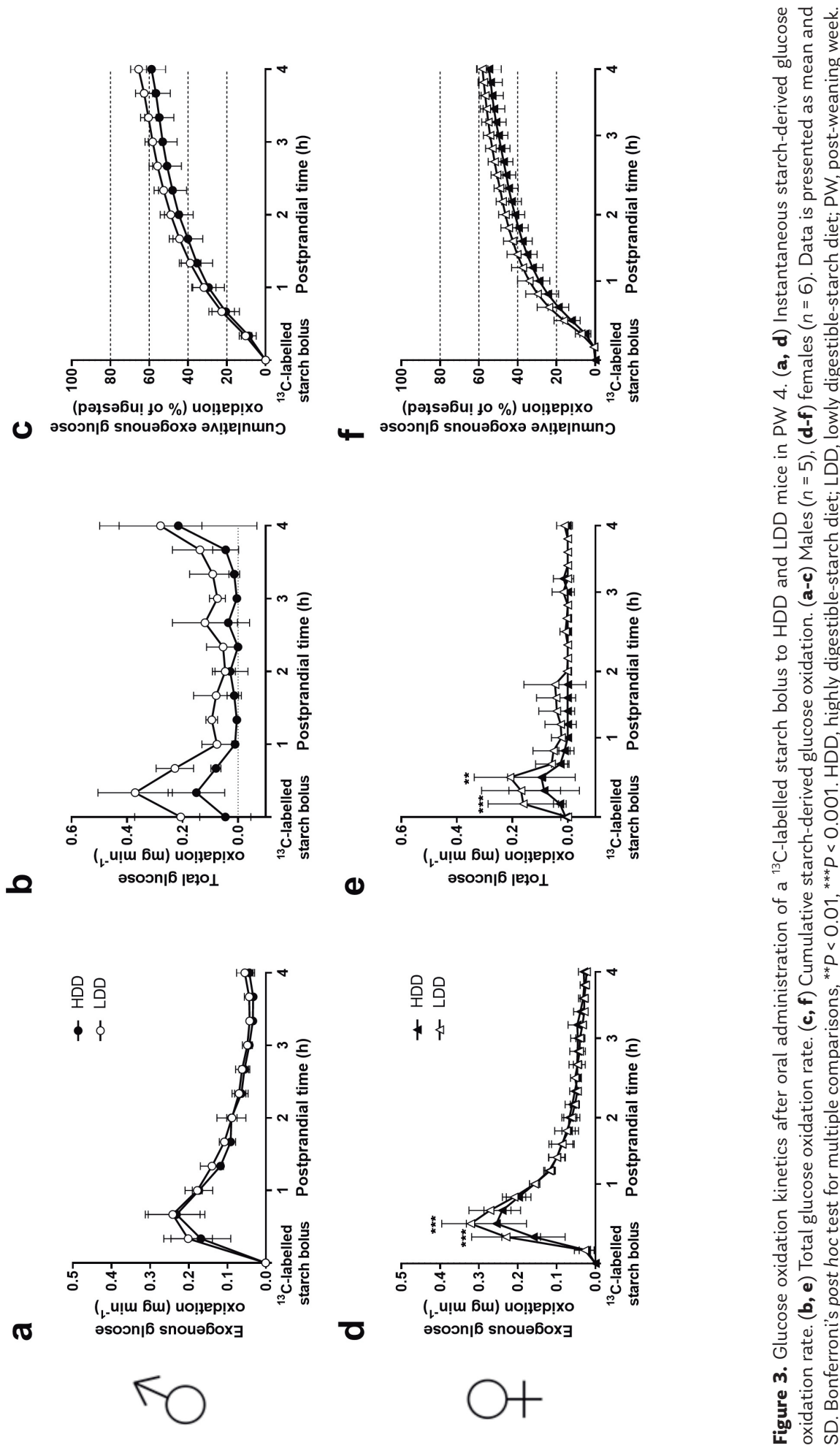

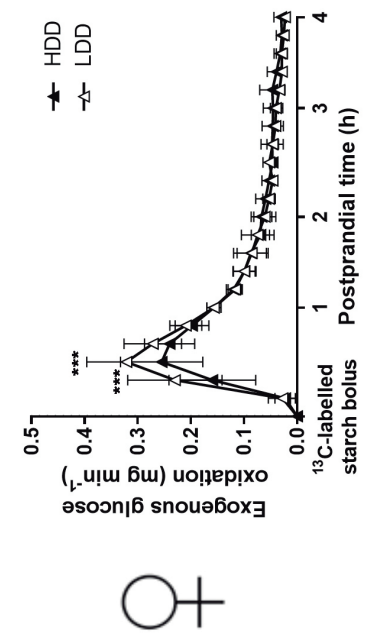

()
A Lowly Digestible-Starch Diet After Weaning Enhances Exogenous Glucose Oxidation Rate in Female, but Not in Male, Mice 
Finally, total glucose oxidation rates were initially higher in LDD females (diet $\times$ time interaction, $P<0.05$; post-weaning diet, $P<0.05$; Figure $3 e$ ). These data indicate that LDD females oxidised starch-derived glucose significantly faster than HDD females in the early postprandial phase on consumption of the starch bolus.

Regarding the cumulative amounts of starch-derived glucose oxidised throughout the $4 \mathrm{~h}$ after administration of the ${ }^{13} \mathrm{C}$-starch bolus, LDD males oxidised more starch over time than HDD males (diet $\times$ time interaction, $P<0.01$; Figure $3 c$ ), while this effect was not observed in females (Figure 3f). A comparison of the total amount oxidised at $4 \mathrm{~h}$ (as percentage of dose administered) across sexes and experimental diets showed significant main effects of sex $(P<0.05)$ and diet $(P<0.05)$, indicating that males oxidised starchderived glucose more extensively than females and that overall LDD mice oxidised more exogenous glucose than HDD mice.

Intestinal amylase activity and hepatic ${ }^{13} \mathrm{C}$ label deposition in females

Two additional analyses were done in the females. First, we tested carbohydrate digestion capacity as a tentative explanation for the higher starch-derived glucose oxidation rates of LDD females. Amylase levels in small intestinal contents collected immediately before and $4 \mathrm{~h}$ after administration of the ${ }^{13} \mathrm{C}$ starch bolus showed that LDD mice had lower pancreatic amylase levels compared to HDD mice, as indicated by an overall effect of $\operatorname{diet}(P<0.001$; Figure 4a). However, after accounting for the larger amount of small intestinal contents in LDD mice (diet, $P<0.05$; Figure $4 \mathrm{~b}$ ), there were not clear differences in pancreatic amylase levels between LDD and HDD females (Figure 4c). Secondly, we examined whether an increased flux of starch-derived glucose to liver could explain the higher oxidation rates seen in LDD females. Before ingestion of the ${ }^{13} \mathrm{C}$ starch bolus, total ${ }^{13} \mathrm{C}$ enrichment in the liver was marginally higher in LDD compared to HDD females [1.0852 (SD 0.0001) us 1.0845 (SD 0.0004) atom\%, respectively; $P=0.053$ ]. Four hours after administration of the starch bolus, LDD females tended to have a higher deposition of the ${ }^{13} \mathrm{C}$ label in liver above baseline compared to HDD females ( $P=0.09$, Figure 5). This represented 6.5 (SD 1.9) and 5.1 (SD 2.2) \% of the ${ }^{13} \mathrm{C}$ label administered to LDD and HDD females, respectively.
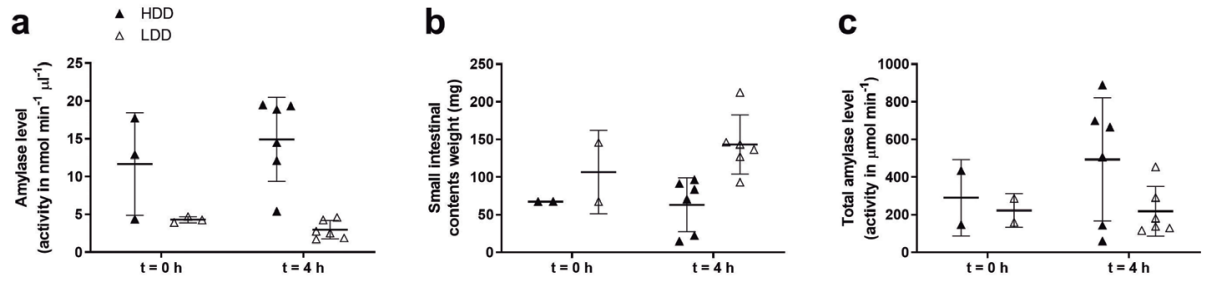

Figure 4. (a) Amylase levels, assayed as activity, per unit of small intestinal contents from HDD and LDD females in PW 4, immediately before $(\mathrm{t}=\mathrm{O} \mathrm{h} ; \mathrm{n}=3)$ and $4 \mathrm{~h}$ after $(\mathrm{t}=4 \mathrm{~h} ; n=6)$ oral administration of a ${ }^{13} \mathrm{C}$-labelled starch bolus. (b) Total weight of small intestinal contents from females in panel (a); note 1 missing value in each group $(t=0 \mathrm{~h})$ that failed to be recorded. (c) Estimated total amylase activity in the entirety of the small intestinal contents, based on data from panels (a) and (b). Data is presented as mean and SD. Student's t-test. HDD, highly digestible-starch diet; LDD, lowly digestible-starch diet; PW, post-weaning week. 


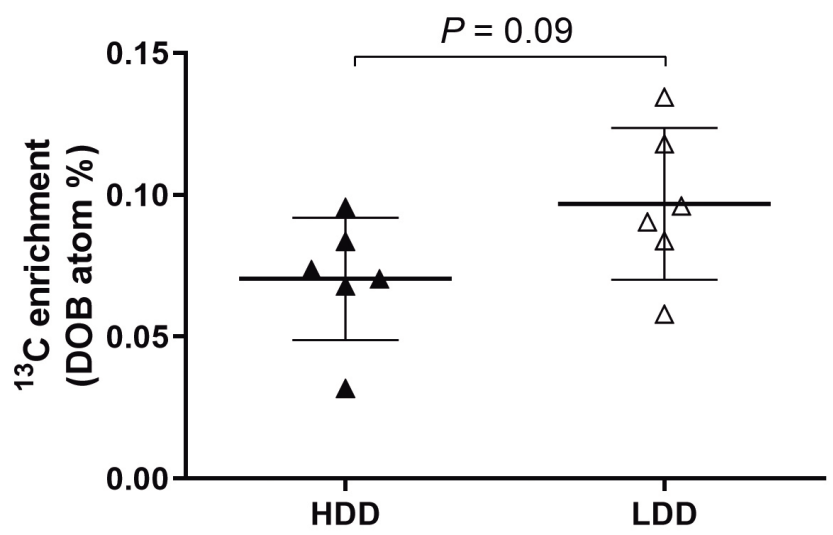

Figure 5. Total ${ }^{13} \mathrm{C}$ enrichment in liver tissue from HDD and LDD females $4 \mathrm{~h}$ after oral administration of a ${ }^{13} \mathrm{C}$-labelled starch bolus in PW 4. Data $(n=6)$ is expressed relative to females that did not receive the ${ }^{13} \mathrm{C}$ starch bolus $(n=3)$ and presented as mean and SD. Student's t-test. DOB, delta over baseline; HDD, highly digestiblestarch diet; LDD, lowly digestible-starch diet; PW, post-weaning week.

\section{Discussion}

We show that female mice exposed for three weeks to an LDD in the immediate postweaning period developed an increased capacity to oxidise exogenous starch-derived glucose compared to females who consumed an HDD, even in light of the unaffected BW and BC by the type of starch. Moreover, the effect of LDD on starch-derived glucose oxidation was less pronounced in male mice, confirming that males and females respond differently to dietary starches.

An important advantage in our study was the use of ${ }^{13} \mathrm{CO}_{2}$ enrichment analysis combined with conventional indirect calorimetry ( $\mathrm{InCa}$ ). It has been shown previously that adult male mice fed a low glycaemic index diet for three weeks have a better capacity only for oxidisation of ingested fat compared to mice on a high glycaemic index $\operatorname{diet}^{10}$. The authors concluded that exogenous glucose oxidation is unaffected by prior exposure to starches of different digestibility, but this was based on a challenge with pure glucose, assessed only qualitatively (by measurement of only ${ }^{13} \mathrm{CO}_{2}$ enrichment, but not of total $\mathrm{CO}_{2}$ production volumes), and the study did not include female mice. We therefore considered it important to focus on the oxidation of the starch molecule in both sexes using a quantitative method. While RER data alone is a well-founded approach to substrate oxidation analysis, the precise distinction of exogenous us endogenous fuels can only be achieved by including ${ }^{13} \mathrm{CO}_{2}$ analysis together with total $\mathrm{CO}_{2}$ determination. This method was particularly useful given the uncertainty about the true nature of the fuels oxidised in the fasted state prior to the administration of the ${ }^{13} \mathrm{C}$ starch bolus, as discussed below. Furthermore, complementing the analysis of exogenous glucose oxidation rates with $\mathrm{O}_{2}$ measurements revealed that the starch bolus affected metabolic rate in LDD and HDD males differently (Figure S3a), offsetting the marginally higher ${ }^{13} \mathrm{CO}_{2}$ enrichment in LDD males (Figure S2a) and ultimately explaining the similar exogenous glucose oxidation rates in LDD us HDD males (Figure 3a). Additionally, it was now possible to quantify the total amounts of starch 
oxidised over the four-hour postprandial period, and this showed that post-weaning LDD increased the oxidative disposal of starch in both sexes. Thus, we verified that the threeweek exposure to LDD and HDD in males had only a minor impact on the oxidation kinetics of ingested starch, despite total carbohydrate oxidation (calculated from RER) remaining higher throughout the starch bolus challenge and the final quantities of starch oxidised being higher in LDD us HDD males.

We confirmed that consuming lowly digestible starches gives lower RER responses compared to highly digestible starches, as was previously seen in mice ${ }^{21,22}$ and similar to the response to a low glycaemic index meal in humans ${ }^{23,24}$. A lower RER is generally interpreted as higher fat oxidation, a process that can be stimulated in skeletal muscle and liver by short-chain fatty acids (SCFA) produced from microbial carbohydrate fermentation ${ }^{2,25}$. Oxidation of SCFA could explain why LDD us HDD males in our study oxidised remarkably different fuel mixtures despite both groups being in the fasted state, when whole body maximal FA oxidation would be indicated by lowest RER levels. However, it is somewhat surprising that LDD and HDD females had a similar fasting RER. Caecal and colonic digesta weights before the administration of the starch bolus where higher in LDD mice irrespective of sex (data not shown), but the quantities and fluxes of SCFA were not determined in this study. Speculatively, a similar fasting RER in LDD and HDD females, but not in males, could be attributed to the sexually dimorphic response to short-term fasting, with females favouring lipogenesis from amino acids ${ }^{26}$. Thus, lipogenesis from protein (respiratory quotient $\left.=1.20\right)^{27}$ could mask the influences of SCFA oxidation or signalling on RER and result in a similar fasting RER in LDD and HDD females.

An increased exogenous starch-derived oxidation could reflect a higher capacity for carbohydrate digestion, with the potential to cause obesity ${ }^{28}$. This is not a likely implication from our study, since luminal amylase levels were not higher in LDD us HDD females, while exogenous glucose oxidation rates were higher in LDD females. Further, the cause of the increased starch derived oxidation remains unclear. About $57 \%$ of the ${ }^{13} \mathrm{C}$ label was recovered as ${ }^{13} \mathrm{CO}_{2}$ by $4 \mathrm{~h}$ after ingesting the starch bolus, and only $6 \%$ was recovered in liver tissue. We assumed the ${ }^{13} \mathrm{CO}_{2}$ to reflect direct splanchnic oxidation of glucose released from digested starch. However, a fraction of the starch may have also been fermented, producing ${ }^{13} \mathrm{CO}_{2}$ from the fermentation itself ${ }^{29}$, or from oxidation of ${ }^{13}$ C-labelled SCFA by the host. This, however, is considered unlikely, because the starch bolus was gelatinised by heating in water, a process that facilitates digestion by amylase ${ }^{30}$, and because $\mathrm{H}_{2}$ production did not accompany ${ }^{13} \mathrm{CO}_{2}$ appearance (as observed in humans consuming resistant starch ${ }^{31}$ ) in our study. Since liver lipid content is especially susceptible to interventions with starches ${ }^{10,32,33}$, differences in hepatic deposition of the ${ }^{13} \mathrm{C}$ label in LDD vs HDD females could have provided an alternative explanation. Although we observed a trend in LDD females to have a higher total ${ }^{13} \mathrm{C}$ enrichment, the amount of label recovered in the liver was only $6 \%$. Further interpretation is subject to knowing the exact metabolites that are enriched in this compartment, which could be mainly glycogen or, alternatively, triglycerides synthesised from starch-derived glucose. An explanation may also be provided by the fate of the remaining $37 \%$ of the ${ }^{13} \mathrm{C}$ label. This may be differently distributed in other organs in LDD and HDD mice. For instance, higher insulin-stimulated 


\section{CHAPTER 4}

glucose uptake ${ }^{32}$, and higher glucose oxidation and lower synthesis of lipids from glucose ${ }^{34}$, have all been observed in primary adipocytes isolated from epididymal WAT after dietary interventions with lowly us highly digestible starches.

Shared responses to LDD us HDD in both females and males included increased energy intake and higher $\mathrm{H}_{2}$ production (Table 2 and Figure 1) by LDD, confirming previous findings of $\mathrm{H}_{2}$ production in a similar setting ${ }^{12}$. Moreover, it confirmed that the digestible energy density between the LDD and the HDD was different and that a larger proportion of the carbohydrate fraction in the diet was utilised by the gut microbiota upon LDD feeding. In addition, we have shown that the changes in bacterial community structure after three weeks on LDD us HDD were not significantly affected by sex ${ }^{12}$. These common responses between sexes are in stark contrast with the higher capacity to oxidize exogenous starch seen in females LDD us HDD. It is tempting to speculate that this sexually dimorphic response to starch may be associated with the higher insulin sensitivity of females ${ }^{35,36}$ and perhaps mediated by the gut microbiota, since some gut microbial metabolites may be processed differently by females and males $^{37}$.

All in all, the use of InCa with additional gas sensors $\left({ }^{13} \mathrm{CO}_{2},{ }^{12} \mathrm{CO}_{2}\right.$, and $\left.\mathrm{H}_{2}\right)$ helped us recognise important effects of starch digestibility shared by females and males and those that are sex-dependent, and is testament to the value of extended InCa systems for the refinement of animal research. Further, the absence of significant effects of LDD us HDD on $\mathrm{BW}$ and $\mathrm{BC}$, and on $\mathrm{H}_{2}$ production, amylase levels, and total ${ }^{13} \mathrm{C}$ label deposition in liver after ingestion of a starch bolus, suggested that the higher capacity of LDD females to oxidise starch stems from differences in hepatocellular metabolism or may lie in other organ systems.

\section{Conclusions}

Female mice fed a lowly digestible starch post-weaning diet developed a better capacity to oxidise starch-derived glucose compared to females on a highly digestible starch diet. This effect was only marginal in male mice. Our results suggest that starch digestibility could have different consequences for metabolic health in females and males and should be taken into account when formulating health recommendations for carbohydrate quality.

\section{Author Contributions}

J.M.S.F.-C., J.K., and E.M.v.S. conceptualised the work and designed the studies. J.M.S.F.-C. and E.M.v.S. obtained ethical approval for the mouse experiment. L.M.S.B. and H.J.M.S. contributed to the planning and preparation of the mouse experiment. N.B. contributed to the design of the ${ }^{13} \mathrm{C}$ starch bolus. J.M.S.F.-C. acquired the data. J.M.S.F.-C., J.K., and E.M.v.S. analysed and interpreted the data. J.M.S.F.-C. drafted the manuscript and L.M.S.B., N.B., A.O., J.K., and E.M.v.S. revised it critically for important intellectual content. All authors approved the final version of the manuscript. 


\section{Acknowledgements}

We thank Ries de Visser (IsoLife) for his technical advice regarding the use of ${ }^{13} \mathrm{C}$-labelled starch. We also thank MSc student Farya Mubarik and the personnel at the CARUS animal facility for their assistance in mouse care-taking. 


\section{References}

1 Campbell, G. J., Senior, A. M. \& Bell-Anderson, K. S. Metabolic effects of high glycaemic index diets: a systematic review and meta-analysis of feeding studies in mice and rats. Nutrients 9, 646 (2017).

2 Higgins, J. A. et al. Resistant starch consumption promotes lipid oxidation. Nutr. Metab. 1, 8 (2004).

3 Johnston, K. L., Thomas, E. L., Bell, J. D., Frost, G. S. \& Robertson, M. D. Resistant starch improves insulin sensitivity in metabolic syndrome. Diabet. Med. 27, 391-397 (2010).

4 Robertson, M. D., Bickerton, A. S., Dennis, A. L., Vidal, H. \& Frayn, K. N. Insulin-sensitizing effects of dietary resistant starch and effects on skeletal muscle and adipose tissue metabolism. Am. J. Clin. Nutr. 82, 559-567 (2005).

$5 \quad$ Zhang, L. et al. Metabolic phenotypes and the gut microbiota in response to dietary resistant starch type 2 in normal-weight subjects: a randomized crossover trial. Sci. Rep. 9, 4736 (2019).

Robertson, M. D. Dietary-resistant starch and glucose metabolism. Curr. Opin. Clin. Nutr. Metab. Care 15, 362-367 (2012).

Peterson, C. M. et al. Effect of $12 \mathrm{wk}$ of resistant starch supplementation on cardiometabolic risk factors in adults with prediabetes: a randomized controlled trial. Am. J. Clin. Nutr. 108, 492-501 (2018).

Robertson, M. D., Livesey, G. \& Mathers, J. C. Quantitative kinetics of glucose appearance and disposal following a C-labelled starch-rich meal: comparison of male and female subjects. Brit. J. Nutr. 87, 569 (2007).

Fernandez-Calleja, J. M. S. et al. Direct and long-term metabolic consequences of lowly vs. highlydigestible starch in the early post-weaning diet of mice. Nutrients 10,1788 (2018).

Isken, F. et al. Impairment of fat oxidation under high- vs. low-glycemic index diet occurs before the development of an obese phenotype. Am. J. Physiol. Endocrinol. Metab. 298, E287-295 (2010).

Hoevenaars, F. P. et al. BIOCLAIMS standard diet (BIOsd): a reference diet for nutritional physiology. Genes Nutr. 7, 399-404 (2012).

Fernández-Calleja, J. M. S. et al. Non-invasive continuous real-time in vivo analysis of microbial hydrogen production shows adaptation to fermentable carbohydrates in mice. Sci. Rep. 8, 15351 (2018).

Duivenvoorde, L. P. et al. A difference in fatty acid composition of isocaloric high-fat diets alters metabolic flexibility in male C57BL/6]OlaHsd mice. PLoS One 10, e0128515 (2015).

Peronnet, F. \& Massicotte, D. Table of nonprotein respiratory quotient: an update. Can. J. Sport Sci. 16, 23-29 (1991).

Delarue, J. et al. The contribution of naturally labelled ${ }^{13} \mathrm{C}$ fructose to glucose appearance in humans. Diabetologia 36, 338-345 (1993).

Englyst, H. N., Kingman, S. M. \& Cummings, J. H. Classification and measurement of nutritionally important starch fractions. Eur. J. Clin. Nutr. 46 Suppl 2, S33-50 (1992).

Petersen, M. C., Vatner, D. F. \& Shulman, G. I. Regulation of hepatic glucose metabolism in health and disease. Nat. Reu. Endocrinol. 13, 572-587 (2017).

Casado, J., Fernandez-Lopez, J. A., Argiles, M. J. \& Alemany, M. Role of the rat liver in the disposal of a glucose gavage. Mol. Cell. Biochem. 113, 33-41 (1992).

Warner, D., Ferreira, L. M., Breuer, M. J., Dijkstra, J. \& Pellikaan, W. F. Stable isotope labeled n-alkanes to assess digesta passage kinetics through the digestive tract of ruminants. PLoS One 8, e75496 (2013). 
Scribner, K. B., Pawlak, D. B., Aubin, C. M., Majzoub, J. A. \& Ludwig, D. S. Long-term effects of dietary glycemic index on adiposity, energy metabolism, and physical activity in mice. Am. J. Physiol. Endocrinol. Metab. 295, E1126-1131 (2008).

Zhou, J. et al. Failure to ferment dietary resistant starch in specific mouse models of obesity results in no body fat loss. J. Agric. Food Chem. 57, 8844-8851 (2009).

Clapp, J. F. \& Lopez, B. Low-versus high-glycemic index diets in women: effects on caloric requirement, substrate utilization and insulin sensitivity. Metab. Syndr. Relat. Disord. 5, 231-242 (2007).

Kaur, B., Quek Yu Chin, R., Camps, S. \& Henry, C. J. The impact of a low glycaemic index (GI) diet on simultaneous measurements of blood glucose and fat oxidation: a whole body calorimetric study. J. Clin. Transl. Endocrinol. 4, 45-52 (2016). and T2DM. Nat. Reu. Endocrinol. 15, 261-273 (2019).

Della Torre, S. et al. Short-term fasting reveals amino acid metabolism as a major sex-discriminating factor in the liver. Cell Metab. 28, 256-267 e255 (2018).

Ferrannini, E. The theoretical bases of indirect calorimetry: A review. Metabolism 37, 287-301 (1988).

Azzout-Marniche, D. et al. High pancreatic amylase expression promotes adiposity in obesity-prone carbohydrate-sensitive rats. J. Nutr. (2019).

Miller, T. L. \& Wolin, M. J. Fermentations by saccharolytic intestinal bacteria. Am. J. Clin. Nutr. 32 164-172 (1979). assess starch digestion and fermentation in humans. J. Nutr. 134, 1193-1196 (2004).

So, P. W. et al. Impact of resistant starch on body fat patterning and central appetite regulation. PLoS One 2, e1309 (2007).

van Schothorst, E. M., Bunschoten, A., Schrauwen, P., Mensink, R. P. \& Keijer, J. Effects of a highfat, low- versus high-glycemic index diet: retardation of insulin resistance involves adipose tissue modulation. FASEB ]. 23, 1092-1101 (2009).

Kabir, M. et al. Dietary amylose-amylopectin starch content affects glucose and lipid metabolism in adipocytes of normal and diabetic rats. J. Nutr. 128, 35-43 (1998).

Ingvorsen, C., Karp, N. A. \& Lelliott, C. J. The role of sex and body weight on the metabolic effects of high-fat diet in C57BL/6N mice. Nutr. Diabetes 7, e261 (2017). adiposity and metabolism in mice. Endocrinology 154, 1092-1104 (2013). 


\section{Supplementary Information}

a

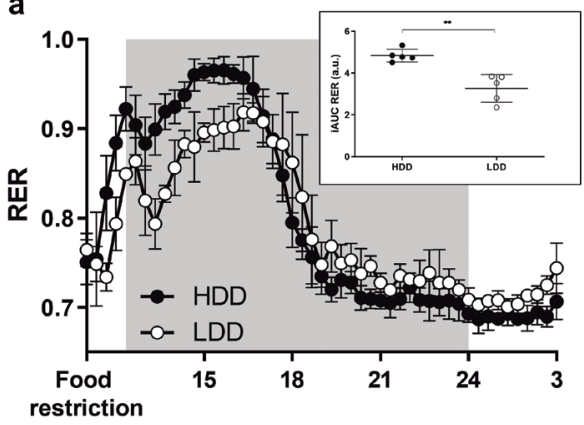

b

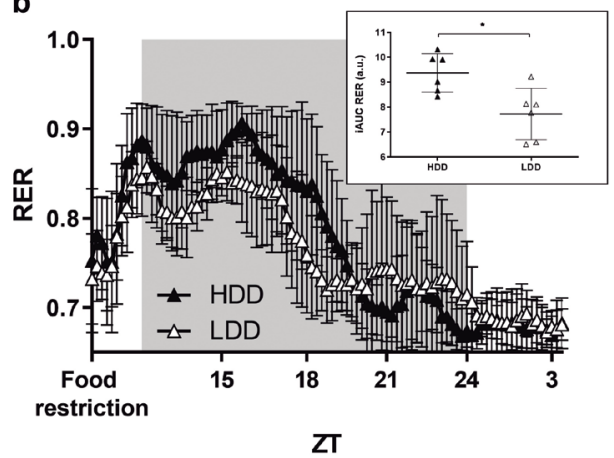

Figure S1. Whole-body substrate oxidation during food restriction in HDD and LDD mice in PW 4. (a) Males $(n=5)$, (b) females $(n=6)$. Approximately $2 \mathrm{~h}$ before DP, all mice received a single $1.2 \mathrm{~g}$ pellet of HDD or LDD, respectively ("food restriction"). Shaded areas represent the dark phase. Insets in panels (a) and (b) show the iAUC of the RER response from the moment of food restriction until just before the administration of the ${ }^{13} \mathrm{C}$ starch bolus. Data is presented as mean and SD. Student's t-test, ${ }^{*} P<0.05,{ }^{* *} P<0.005$. DP, dark phase; HDD, highly digestible-starch diet; iAUC; incremental area under the curve; LDD, lowly digestible-starch diet; PW, postweaning-week; ZT, Zeitgeber time.
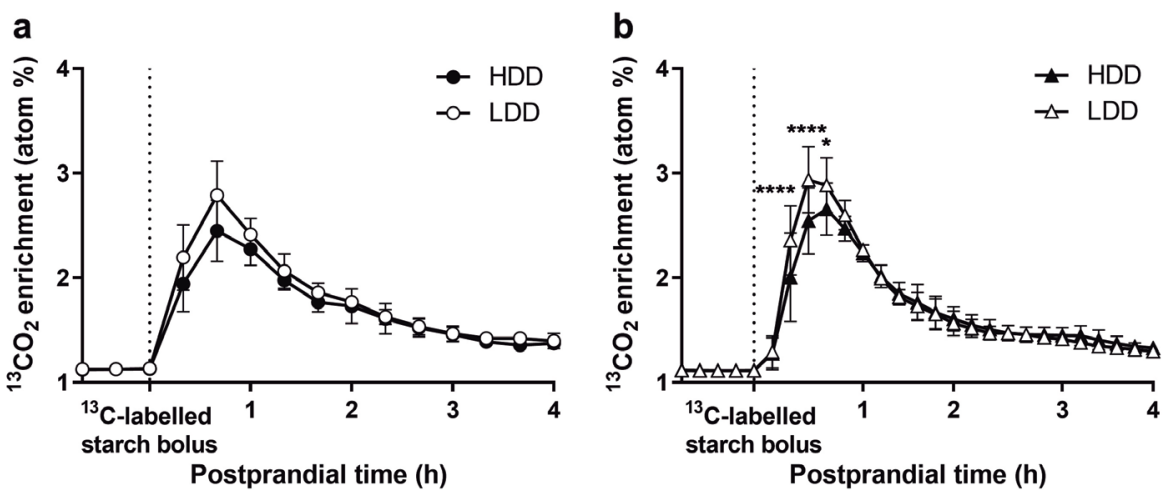

Figure S2. Instantaneous recovery of ${ }^{13} \mathrm{C}$ label as ${ }^{13} \mathrm{CO}_{2}$ after oral administration of a ${ }^{13} \mathrm{C}$-labelled starch bolus to HDD and LDD mice in PW 4. (a) Males $(n=5)$, (b) females $(n=6)$. Data is presented as mean and SD. Bonferroni's post hoc test for multiple comparisons, ${ }^{*} P<0.05,{ }^{* * * *} P<0.0001$. HDD, highly digestible-starch diet; LDD, lowly digestible-starch diet; PW, post-weaning week. 
a

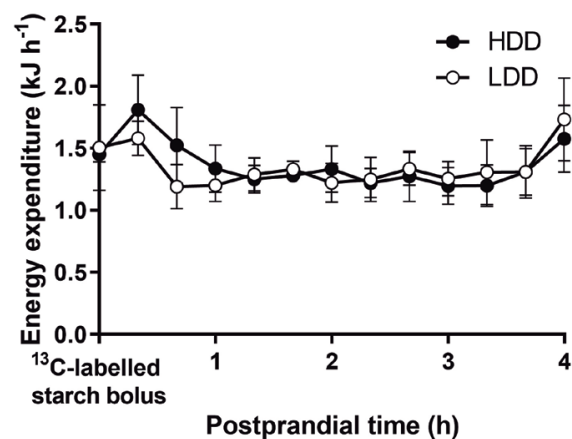

b

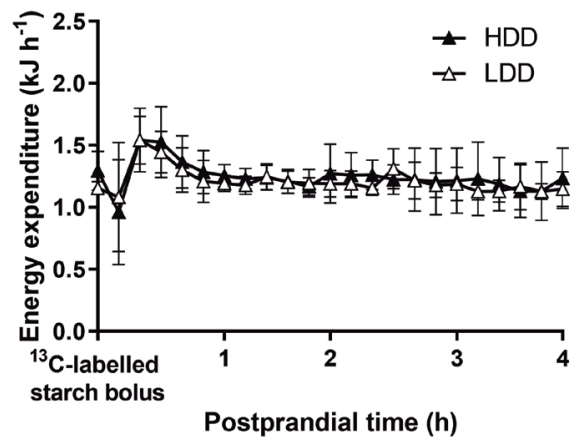

Figure S3. Energy expenditure after oral administration of a ${ }^{13} \mathrm{C}$-labelled starch bolus to HDD and LDD mice in PW 4. (a) Males $(n=5)$, (b) females $(n=6)$. Data is presented as mean and SD. HDD, highly digestible-starch diet; LDD, lowly digestible-starch diet; PW, post-weaning week. 



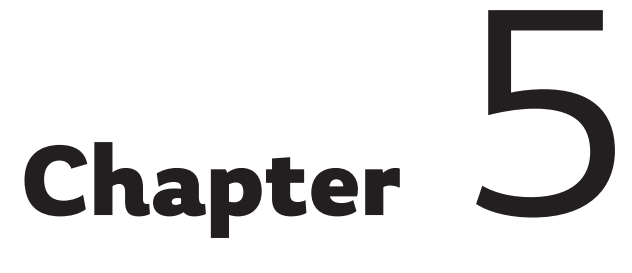

Direct and Long-Term Metabolic Consequences of Lowly vs. Highly-Digestible Starch in the Early Post-Weaning Diet of Mice

José M. S. Fernández-Calleja

Lianne M. S. Bouwman

Hans J. M. Swarts

Annemarie Oosting

Jaap Keijer

Evert M. van Schothorst

Nutrients (2018) 10:1788 DOI: 10.3390/nu10111788 



\section{Abstract}

Starches of low and high digestibility have different metabolic effects. Here, we examined whether this gives differential metabolic programming when fed in the immediate postweaning period. Chow-fed mice were time-mated, and their nests were standardized and cross-fostered at postnatal days 1-2. After postnatal week (PW) 3, individually housed female and male offspring were switched to a lowly-digestible (LDD) or highly-digestible starch diet (HDD) for three weeks. All of the mice received the same high-fat diet (HFD) for nine weeks thereafter. Energy and substrate metabolism and carbohydrate fermentation were studied at the end of the HDD/LDD and HFD periods by extended indirect calorimetry. Glucose tolerance (PW 11) and metabolic flexibility (PW14) were analyzed. Directly in response to the LDD versus the HDD, females showed smaller adipocytes with less crown-like structures in gonadal white adipose tissue, while males had a lower fat mass and higher whole body fat oxidation levels. Both LDD-fed females and males showed an enlarged intestinal tract. Although most of the phenotypical differences disappeared in adulthood in both sexes, females exposed to LDD versus HDD in the early post-weaning period showed improved metabolic flexibility in adulthood. Cumulatively, these results suggest that the type of starch introduced after weaning could, at least in females, program later-life health.

Keywords: Glycemic index, nutrition, amylose, amylopectin, carbohydrates, C57BL mice, sexual dimorphism, indirect calorimetry, adipose tissue, metabolic flexibility. 


\section{Introduction}

Early life experiences in critical periods during prenatal and postnatal development have the potential to program metabolic health later in life. While early-life nutrition has been identified as a major environmental condition inducing long-lasting effects in the organism, the optimal diet to promote a healthy life from conception to adulthood is still ill-defined. Much emphasis has been placed on nutritional interventions prenatally and during infancy, since this is considered the period of maximal developmental plasticity. However, it is recognized that the critical development period extends after infancy in some organs and systems ${ }^{1}$.

Early life exposure to different qualities and quantities of protein and lipids has been shown to have a lasting impact on adult metabolic health ${ }^{2-5}$. Dietary carbohydrates may also have a role in programming of later-life metabolic health, as both quality and quantity could provide cues for disease development, treatment, and management. A high intake of low glycemic index (GI) foods is associated with improved health outcomes in both adults and children ${ }^{6,7}$. Using highly defined diets, with only the type of starch being different, we have previously shown that a low versus high GI diet delayed obesity-associated disease development in adult mice ${ }^{8}$. Moreover, the low versus high $\mathrm{Gl}$ diet induced intestinal microbiota hydrogen production in young and adult mice ${ }^{9}$. Thus, the digestibility of starches provides them with different nutritional properties for both the host and the intestinal microbiota through fermentation ${ }^{10}$.

The introduction of solid foods to gradually replace breast milk-or weaning-is a crucial period in the life course. In humans, this period also represents the transition from a highfat to a high-carbohydrate content in the $\operatorname{diet}^{11}$. Importantly, it is during this transition that decisive interactions between the organism and the gut microbiota are being established ${ }^{12}$. Current evidence-based recommendations for complementary feeding are mainly focused on the time of introduction of allergenic foods and solids, with particular attention to protein and $\mathrm{fat}^{13}$. However, the rationale for choosing carbohydrates in complementary foods is only based on the development of taste preferences and the prevention of caries ${ }^{13}$. Clearly, carbohydrate intake during early life should also be examined from a metabolic health perspective ${ }^{14}$.

The recommendations of the joint Food and Agriculture Organization of the United Nations and the World Health Organization (FAO/WHO) expert consultation for carbohydrate intake are virtually the same for all individuals over two years of age\{, \#1267; Food, 1998 $\# 1349\}^{15}$, and did not substantially change over the course of 10 years due to limited new data. Rodent models are instrumental in developmental programming research due to short gestation and maturity periods and the possibility of exploring molecular mechanisms in ways that would be impractical or unethical in humans ${ }^{16}$.

The strongest evidence for programming by early-life carbohydrates has been obtained from studies in rats fed a high-carbohydrate milk formula during the suckling period ${ }^{17}$. Another stepping stone in this area is the work of Gugusheff et al. ${ }^{18}$, which suggested that starches of different digestibility consumed by dams during the perinatal period as well as their 
offspring until early adulthood had long-term consequences for metabolic health. However, due to the study design, it was impossible to distinguish between the direct effects and metabolic programming effects, as offspring consumed these intervention diets until the end of the study.

Several rodent studies incorporating the post-weaning period of growth and development into the programming model have demonstrated metabolic programming by dietary lipids $^{19,20}$, protein ${ }^{21}$, calcium ${ }^{22}$, and the fat:carbohydrate ratio ${ }^{23}$, and a lack of differential programming effects in the case of glucose versus fructose ${ }^{24}$.

In this study, we investigated the long-term effects of starches of different digestibility consumed only during the specific window from weaning until mid-adolescence, on adult metabolic health. We focused on the general aspects of the resulting phenotypes, with an emphasis on metabolic function, e.g., body composition development and whole body metabolism, in both female and male mice. We hypothesized that a lowly-digestible starch post-weaning diet would be protective against the metabolic impairment induced by a high-fat diet during adulthood. We concluded that the early post-weaning period is indeed amenable to metabolic programming by dietary starches, since females consuming a lowlydigestible versus highly-digestible starch diet in the early post-weaning period had a better metabolic flexibility in adulthood.

\section{Materials and Methods}

\section{Animal model}

The study was approved by the Animal Experiment Committee of Wageningen University (DEC 2014085) and performed in accordance to European Union (EU) directives 86/609/ EEC and 2010/63/EU. All of the mice (C57BL/6]RccHsd; Harlan Laboratories BV, Horst, The Netherlands) were housed in polycarbonate type II cages enriched with wood chips and wood shavings, with free access to drinking water and food, at $23 \pm 1{ }^{\circ} \mathrm{C}, 50 \pm 5 \%$ humidity, on a 12-h light/dark cycle. A schematic overview of the study design is shown in Figure 1. Female and male mice (17-19 weeks old) were fed standard rodent chow (26\% $\mathrm{w} / \mathrm{w}$ protein, $38.8 \% \mathrm{w} / \mathrm{w}$ starch, $4.6 \% \mathrm{w} / \mathrm{w}$ sugar, $6.5 \% \mathrm{w} / \mathrm{w}$ fat; AM-II, AB Diets, Woerden, The Netherlands) and time-mated. At postnatal days 1-2, offspring were redistributed across foster dams to produce standardized litters of six pups and a sex ratio of 3:3 or 4:2. At the end of postnatal week (PW 3), all of the mice were housed individually and assigned either a highly-digestible starch diet (HDD; Research Diet Services, Wijk bij Duurstede, The Netherlands; details are described below) or a lowly-digestible starch diet (LDD; Research Diet Services) stratified according to body weight (BW; $n=24$ per sex and diet; one male on LDD was excluded due to incisor malocclusion). At the end of PW 6, a subgroup of mice of each sex and dietary group was sacrificed and white adipose tissue (WAT) from gonadal and mesenteric origin, liver, pancreas, and intestine and its contents, were dissected, snapfrozen in liquid nitrogen, and stored at $-80{ }^{\circ} \mathrm{C}$ until further analysis. The remaining mice ( $n=12$ per sex and diet) were switched to a high-fat diet (HFD, Research Diet Services $\mathrm{BV}$ ) and continued on this diet until sacrifice in PW 15. Food intake (FI) was determined weekly. BW and body composition (BC; EchoMRI 100V, EchoMedical Systems, Houston, 
TX, USA) were determined weekly from PW 4-6 and biweekly from PW 7-15. Two indirect calorimetry (InCa) measurements (PW 6 and PW 14) and an oral glucose tolerance test (OGTT; PW 11) were carried out as described below.

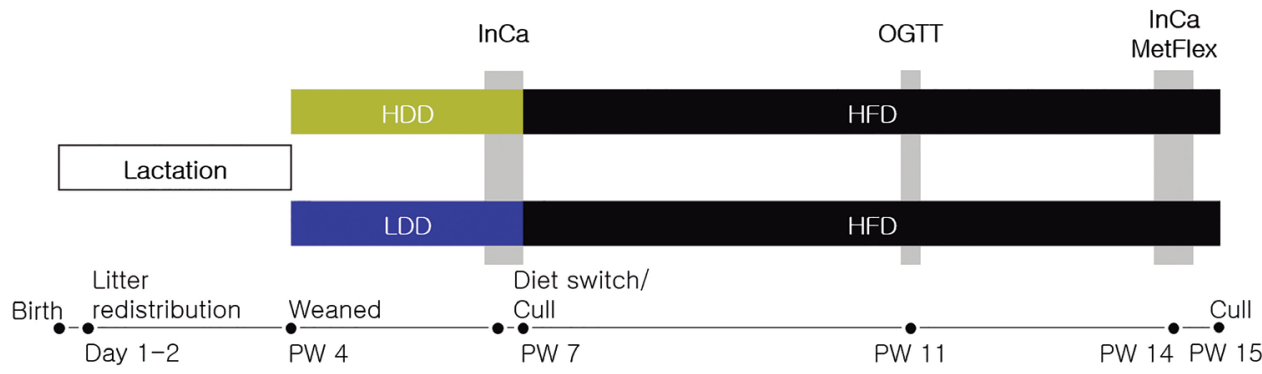

Figure 1. Experimental design. Female and male mice fed standard rodent chow were time-mated and their offspring were redistributed at postnatal day 1-2 to standardized nests. At the end of postnatal week (PW) 3, after weaning, the pups were individually housed and fed either a highly-digestible starch diet (HDD) or a lowlydigestible starch diet (LDD). In PW 6, all of the mice had their basal metabolic phenotype assessed by indirect calorimetry $(\mathrm{InCa})$. A subgroup of animals of each diet and sex was dissected at the end of PW 6 to further assess the direct effects of the post-weaning dietary intervention. The remaining mice were switched to a high-fat diet (HFD) from PW 7 onwards to study metabolic programming in an obesogenic environment. In PW 11, mice underwent an oral glucose tolerance test (OGTT). At the end of HFD-feeding, basal metabolism was measured, and metabolic flexibility (MetFlex) was assessed by InCa. Mice were culled at the end of PW 15, and their blood and tissues were harvested for further analysis.

\section{Experimental diets}

All of the experimental diets were based on the BIOCLAIMS standard diet ${ }^{25}$. Both HDD and LDD contained 20 energy percentage (en\%) protein, 55 en $\%$ carbohydrates, and 25 en\% fat, with highly-digestible or lowly-digestible starches as the sole difference and source of available carbohydrate (Cargill, Sas van Gent, The Netherlands; incorporated into pelleted diets by Research Diet Services), as published ${ }^{9}$. The HFD contained 20 en\% protein, 40 en\% carbohydrates, and 40 en $\%$ fat $^{26}$. Detailed diet formulations are shown in Table 1.

\section{Oral glucose tolerance test}

An OGTT was performed five hours after food withdrawal in PW 11 by the administration of glucose $\left(2 \mathrm{~g} \mathrm{~kg}^{-1} \mathrm{BW}\right)$ by oral gavage as published ${ }^{24}$.

Indirect calorimetry (InCa) and metabolic flexibility

The general procedure for indirect calorimetry measurements has been described previously ${ }^{24}$, with minor adjustments. After an 18-h adaptation period, the energy expenditure (EE), respiratory exchange ratio (RER), locomotor activity, and food intake were measured in a PhenoMaster indirect calorimetry system (TSE Systems GmbH, Bad Homburg, Germany), which was extended with hydrogen $\left(\mathrm{H}_{2}\right)$ and methane sensors for

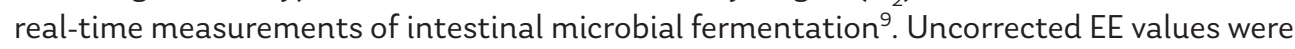


used, since lean mass (LM) was not significantly different between dietary groups directly before or after each InCa period. To assess metabolic flexibility (PW 14), mice were fed a restricted amount of HFD ( $1.1 \mathrm{~g}$, which is equivalent to about $55 \%$ of average food intake during the dark phase) two hours prior to the dark phase to induce a fasting state the next morning. Approximately one hour before the following dark phase, all of the mice were given a meal challenge (HDD) ad libitum, and measurements continued until the following light phase. The switch from predominantly fat oxidation (RER $=0.7$ ) toward net carbohydrate oxidation ( $R E R=1.0$ ) upon refeeding was used as a measure of metabolic flexibility ${ }^{27}$. A selection of data obtained from animals at PW 6 has been previously reported: EE, RER, and $\mathrm{H}_{2}$ production ${ }^{9}$.

Table 1. Composition of the experimental diets.

\begin{tabular}{|c|c|c|c|}
\hline & HDD & LDD & HFD \\
\hline Casein & 212.2 & 212.0 & 233.5 \\
\hline L-Cysteine & 3.0 & 3.0 & 3.0 \\
\hline Amylose mix (AmyloGel 03003) ${ }^{1}$ & 0.0 & 568.6 & 0.0 \\
\hline Amylopectin $\left(\mathrm{C}^{*} \mathrm{Gel} 04201\right)^{2}$ & 568.6 & 0.0 & 0.0 \\
\hline Wheat starch & 0.0 & 0.0 & 285.6 \\
\hline Maltodextrin & 0.0 & 0.0 & 100.0 \\
\hline Glucose & 0.0 & 0.0 & 70.0 \\
\hline Coconut oil & 21.4 & 21.4 & 0.0 \\
\hline Sunflower oil & 83.1 & 83.1 & 0.0 \\
\hline Flaxseed oil & 14.2 & 14.2 & 4.0 \\
\hline Palm oil & 0.0 & 0.0 & 206.3 \\
\hline Cholesterol & 0.03 & 0.03 & 0.097 \\
\hline Cellulose & 50.0 & 50.0 & 50.0 \\
\hline Mineral mix (AIN-93G-MX) & 35.0 & 35.0 & 35.0 \\
\hline Vitamin mix (AIN-93-VX) & 10.0 & 10.0 & 10.0 \\
\hline Choline bitartrate & 2.5 & 2.5 & 2.5 \\
\hline Total (g) & 1000.0 & 1000.0 & 1000.0 \\
\hline Gross energy density $\left(\mathrm{kJ} \mathrm{g}^{1}\right)^{3}$ & 18.9 & 19.5 & 20.8 \\
\hline Calculated energy density $\left(\mathrm{kJ} \mathrm{g}^{-1}\right)^{4}$ & 17.9 & 17.9 & 19.8 \\
\hline Protein (energy\%) & 20.1 & 20.1 & 20.0 \\
\hline Carbohydrate (energy\%) & 54.9 & 54.9 & 40.0 \\
\hline Fat (energy\%) & 25.0 & 25.0 & 40.0 \\
\hline
\end{tabular}

All values are in $\mathrm{g} \mathrm{kg}^{-1}$ unless indicated. ${ }^{1} 60 \%$ amylose, $40 \%$ amylopectin (Cargill). ${ }^{2} 100 \%$ amylopectin (Cargill BV). ${ }^{3}$ Determined by bomb calorimetry. ${ }^{4}$ Calculated based on Atwater's nutritional values. HDD: highly-digestible starch diet; HFD: high-fat diet; LDD: lowly-digestible starch diet.

\section{Sacrifice}

At the end of PW 6 and PW 15, mice were deprived of food at the onset of the light phase and decapitated two to six hours thereafter. Blood glucose was measured in duplicate with 
a Freestyle glucose meter (Abbott Diabetes Care, Hoofddorp, The Netherlands). Whole blood was collected in chilled MiniCollect serum tubes (Greiner Bio-One BV, Alphen aan de Rijn, The Netherlands), spun down at $4{ }^{\circ} \mathrm{C}$ for $10 \mathrm{~min}$ at $3000 \times \mathrm{g}$, and the resulting serum aliquoted and stored at $-80^{\circ} \mathrm{C}$. Liver, mesenteric white adipose tissue (mWAT), and pancreas were weighed and snap-frozen in liquid nitrogen. A $\sim 2 \mathrm{~g}$ clip was attached to the distal end of the small intestine and hung next to a ruler to determine the length of the small intestine. Thereafter, the small intestine and colon were each cut longitudinally, rinsed in ice-cold RNase-free phosphate-buffered saline to remove their contents, and weighed separately. Caecum contents were extracted, weighed, and snap-frozen. One pad of gonadal white adipose tissue (gWAT) was snap-frozen; the other pad was weighed, fixated in $4 \%$ paraformaldehyde overnight, and embedded in paraffin. Samples were stored at $-80^{\circ} \mathrm{C}$ until further analysis.

\section{Serum measurements}

Serum levels of insulin, leptin, and adiponectin were determined as described using commercial kits ${ }^{24}$.

\section{Hepatic triglycerides and glycogen content}

Hepatic triglycerides were determined using a commercial kit as described ${ }^{24}$. Part of the same liver lobe was used for glycogen determination based on published protocol ${ }^{28}$ with the following minor adaptations: protein-free and lipid-free extracts were obtained by homogenization of $\sim 100 \mathrm{mg}$ of liver tissue in cold $7 \% \mathrm{HClO}_{4}$, centrifugation at $4{ }^{\circ} \mathrm{C}$ for 15 $\min$ at $1500 \times \mathrm{g}$, and further extraction with petroleum ether. Glycogen concentration in the extracts was determined in triplicate by adding iodine-iodide solution in the presence of $\mathrm{CaCl}_{2}$ (260 $\mu \mathrm{L}$ of reagent added to $10 \mu \mathrm{L}$ of sample), and measuring absorbance at $460 \mathrm{~nm}$.

\section{Gonadal white adipose tissue (gWAT) histology}

The paraffin-embedded gWAT pads of six mice per experimental group were selected to represent the average fat mass (FM) and gWAT weight of the complete group. Paraffin blocks were cut into $5-\mu \mathrm{m}$ thick slices with $150-\mu \mathrm{m}$ separation in between sections to ensure different areas within the tissue could be studied. Four to five sections per animal were used to determine the adipocyte area by hematoxylin-eosin (HE) staining, and the number of macrophages and crown-like structures (CLS, MAC-2 staining), as published ${ }^{26,29}$. All of the parameters were based on 1000 intact adipocytes per animal. The fluorescence of eosin resulting from $\mathrm{HE}$ staining was used to visualize adipocytes with a Leica DM6B microscope equipped with a DFC365FX camera (Leica Microsystems, Wetzlar, Germany), and fluorescent photographs were analyzed using CellProfiler software v. 2.1.1 using the adipocyte pipeline by the Rodeheffer Laboratory to measure cell area ${ }^{30,31}$. Adipocyte diameter was calculated from its area based on a circular shape. 
Quantitative real-time reverse-transcription polymerase chain reaction ( $R T$-qPCR)

Total RNA was isolated from gWAT using TRIzol reagent (Invitrogen, Breda, The Netherlands) as described ${ }^{32}$, and cDNA was synthesized with the iScript cDNA synthesis kit (Bio-Rad Laboratories, Veenendaal, The Netherlands). The expressions of genes involved in macrophage infiltration (chemokine ( $\mathrm{C}-\mathrm{C}$ motif) ligand 2, $\mathrm{Ccl}$; lectin, galactose binding, soluble 3, Lgals3; S100 calcium binding protein A8, S100a8), insulin signalling (insulin receptor substrate 2, Irs2), and lipid metabolism (fatty acid binding protein 4, Fabp4) were analyzed in duplicate by RT-qPCR with iQ SYBR Green Supermix (Bio-Rad). Primers were designed to span exon-exon junctions to prevent the amplification of genomic DNA using the NCBI Primer BLAST tool, and PCR products were run on a gel to confirm amplicon sizes when necessary. Standard curves were constructed with cDNA pooled from all samples, a control containing no cDNA, a negative RT control, and a melt curve at the end of the each run, and included for quality control. In the case of the lowly-expressed Ccl2, Lgals3, and $\mathrm{S100a8}$ transcripts, cDNA was pre-amplified for 10 cycles with SsoAdvanced PreAmp Supermix (Bio-Rad) and the corresponding primers, according to the manufacturer's instructions. Full details of all of the primers can be found in Table S1. Normalized gene expression levels were computed with CFX Manager software, v. 3.1. (Bio-Rad) and used for statistical comparisons.

\section{Data analysis}

Statistical analyses were performed in GraphPad Prism 5.04 (GraphPad, San Diego, CA, USA), and female and male data were analyzed separately. Data was tested using the D'Agostino and Pearson omnibus for normality. Non-normally distributed data was logtransformed and re-tested for normality. Two-tailed comparisons between two groups were made using unpaired Student's $t$-tests or Mann-Whitney $U$-tests for normally and non-normally distributed data, respectively. Other group comparisons were tested with two-way ANOVA (adipocyte size frequency distribution and macrophage infiltration), with repeated measurements for matched time course data (OGTT, RER, and carbohydrate intake during $\ln \mathrm{Ca}$ ) and Bonferroni's post hoc test. Correlations analyses were performed using Pearson correlation on normally distributed data and Spearman correlation for nonnormally distributed data. The incremental area under the curve (iAUC) of glucose during OGTT was also calculated in Prism. Statistical significance was set at $p<0.05$ for all of the comparisons.

\section{Results}

Direct and long-term effects on body weight and body composition by post-weaning starches

Directly after three weeks of consumption of HDD or LDD (PW 4-6), there was no difference in body weight or lean mass between the two groups (Figure 2a,c; Figure S1a,c,d,f). However, males on the HDD developed more fat mass compared to those fed the LDD in this period (Figure 2c; Figure S1e). This was not seen in female mice (Figure 2a; Figure S1b). 
Following the intervention period, all of the mice received nine weeks of HFD feeding. At 15 weeks of age, there were no significant differences seen in body weight or body composition in neither females nor males (Figure $2 b, d$ ).
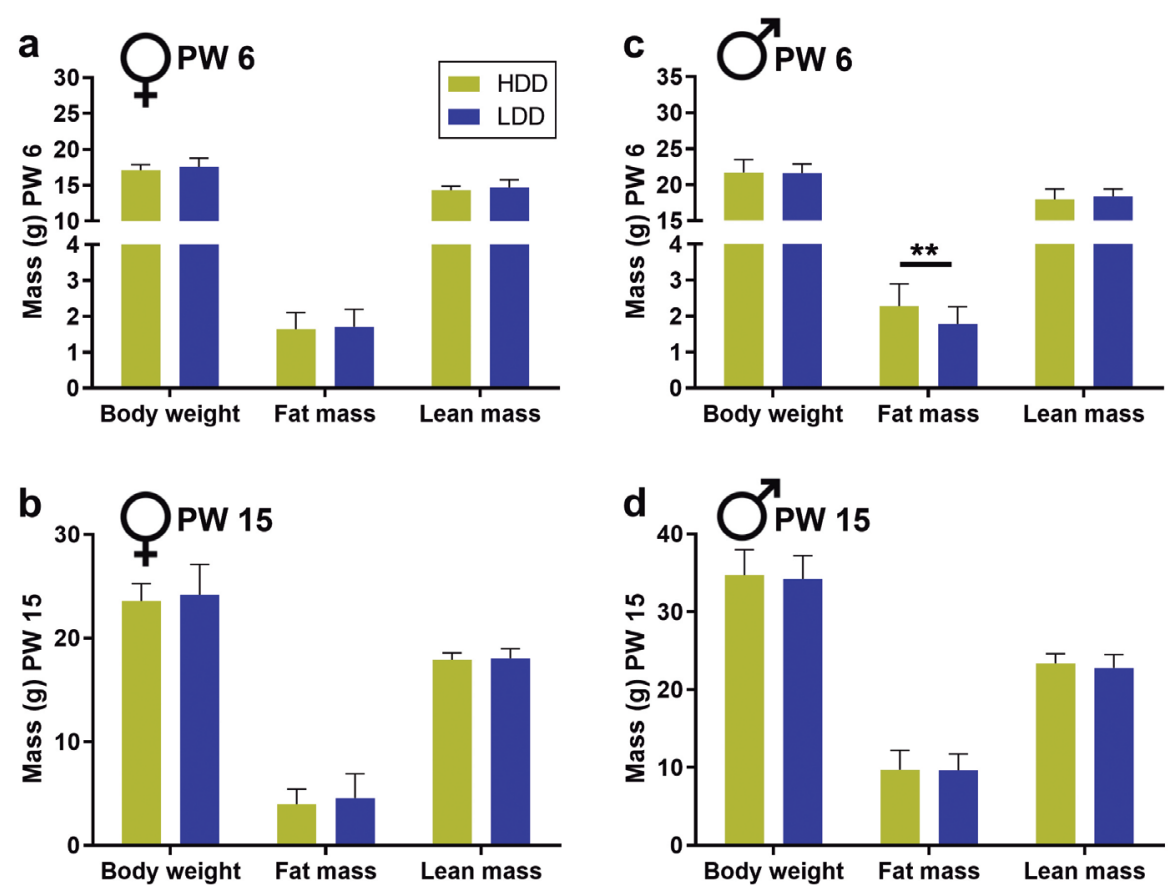

Figure 2. Direct and long-term effects of the type of starch consumed in the early post-weaning period on body weight (BW) and body composition. BW, fat mass (FM), and lean mass (LM) of females directly after exposure to HDD or LDD (a; PW 6, $n=24$ per group), and after nine weeks on a HFD (b; PW 15, $n=12$ per group). The BW, FM, and LM of males after exposure to HDD or LDD (c; PW 6, $n=24$ for HDD and $n=23$ for LDD), and after nine weeks on a HFD (d; PW 15, $n=12$ per group). Note truncated $x$-axis in panels a and $\mathbf{c}$ to enhance visualization. Data shown as mean \pm standard deviation (s.d.). Statistical differences denoted as ${ }^{* *} p \leq 0.01$.

Direct and long-term effects on basal metabolism

Energy expenditure and locomotor activity were not affected by the type of starch neither at the end of the intervention nor upon HFD feeding, for both females and males (Table 2). However, males consuming the LDD showed a lower RER compared to males fed the HDD (Table 2), indicating increased fat over carbohydrate oxidation. This difference in substrate utilization was completely absent in the females. The effects on basal RER that were seen in males disappeared, and thus were not metabolically programmed at the end of the HFD period (Table 2). This cumulatively suggests that basal metabolism, including fuel utilization, is not programmed by the type of starch consumed in the early post-weaning period either in females or males.

A novel parameter that can be measured using our extended indirect calorimetry system is production of the fermentation gases hydrogen $\left(\mathrm{H}_{2}\right)$ and methane ${ }^{9} . \mathrm{H}_{2}$ is exclusively formed by gut microbes as a product of carbohydrate fermentation ${ }^{33}$, and as such represents a 
CHAPTER 5

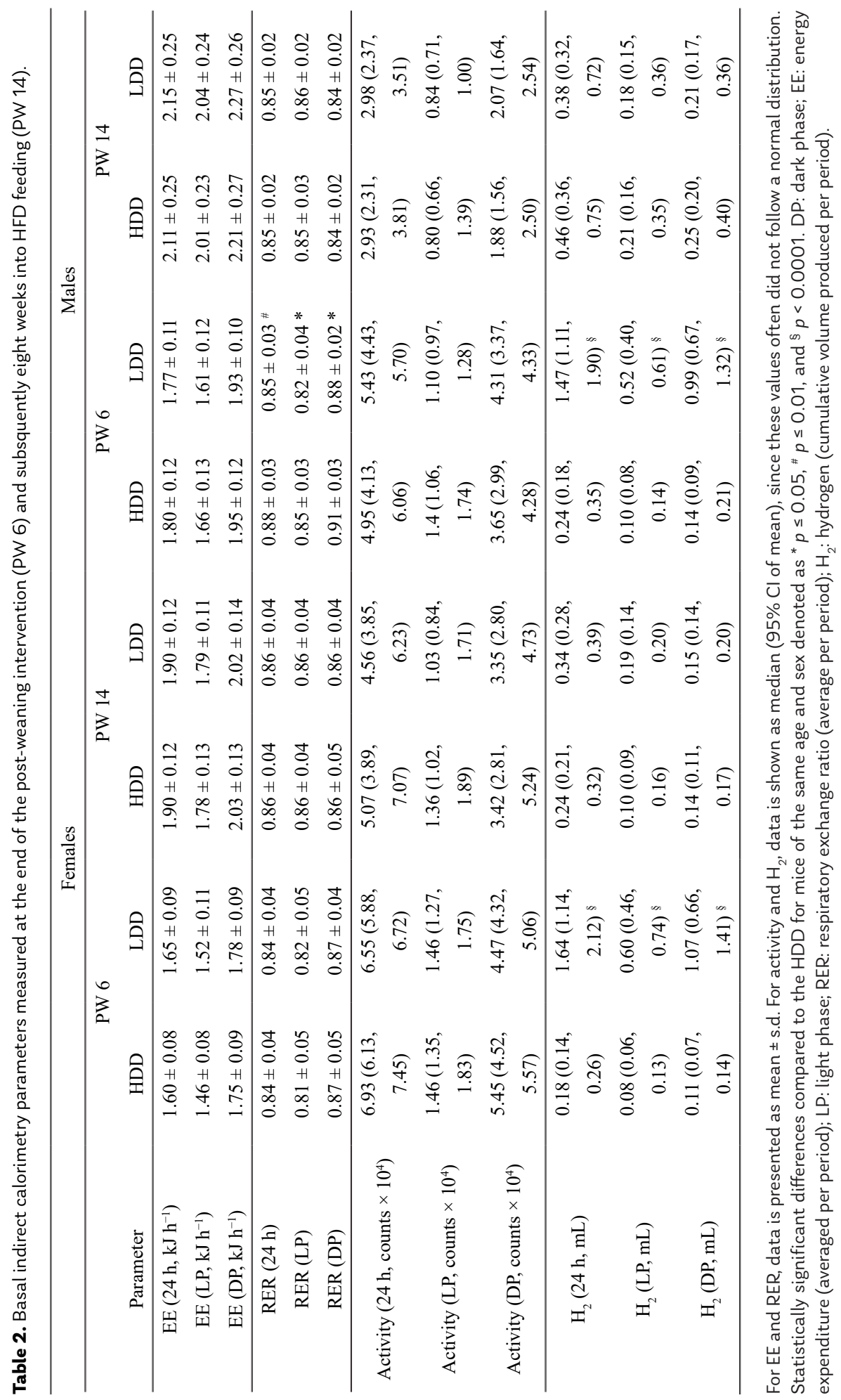


convenient marker for gut microbiota activity. In line with the known differences in digestibility of $40 \%$ amylose and $60 \%$ amylopectin compared with $100 \%$ amylopectin with the food matrix in vitro and in vivo ${ }^{9}$, there were significant differences in $\mathrm{H}_{2}$ output between mice consuming HDD or LDD in both females and males, with LDD mice producing approximately eight times more $\mathrm{H}_{2}$ than $\mathrm{HDD}$ mice over $24 \mathrm{~h}$ (Table 2 , ref. ${ }^{9}$ ). $\mathrm{H}_{2}$ production was relatively low and similar for all of the mice consuming the HFD (Table 2). At the same time, absolute methane levels being at ambient levels indicated an absence of methane production at both the end of the intervention and HFD-feeding periods in any group.

\section{Direct and long-term effects on other physiological parameters at sacrifice}

There were no obvious differences in the weight of metabolic organs and circulating parameters after the early post-weaning intervention or at the end of the HFD period (Table 3). An important exception was the elevated serum leptin concentrations in HDD males in PW 6, which is consistent with the increased fat mass. There were significant differences in the gross energy intake during HDD and LDD feeding, with mice cumulatively ingesting about $0.1 \mathrm{MJ}$ more on the LDD (Table 3); however, this did not lead to a significant difference in body weight or lean mass (Figure 2a,c).

The most remarkable finding in the overall phenotype at sacrifice was the direct effect of the type of starch on intestinal tract morphology. Both the weight and length of the small intestine, and colon weight were increased in females and males consuming the LDD (Table 3). In addition, despite being in the post-absorptive state, cecum contents were increased in females and males fed the LDD (Table 3). At the end of the HFD period, these differences were normalized to values similar to the LDD-fed mice in early life (Table 3).

\section{Long-term effects on glucose tolerance}

The perturbation of homeostasis may allow the detection of subtle or early differences in metabolic phenotypes, particularly those induced by nutritional interventions ${ }^{34}$. We first employed an OGTT to challenge glucose homeostasis in PW 11, when mice had been consuming a HFD for five weeks. No differences were seen at baseline in glycemia (Figure 3a,c) or insulinemia (females: $0.7 \pm 0.1 \mathrm{ng} \mathrm{mL}^{-1}$ versus $0.7 \pm 0.2 \mathrm{ng} \mathrm{mL}^{-1}$; males: $1.6 \pm 0.3 \mathrm{ng} \mathrm{mL}^{-1}$ versus $1.5 \pm 0.4 \mathrm{ng} \mathrm{mL}^{-1}$; mean \pm s.d., HDD and LDD respectively, $n=12$ per group). The glycemic response to the glucose bolus was also similar between groups, with only a trend for a lower iAUC in the LDD females (Figure 3b,d).

Long-term effects on metabolic flexibility

In contrast to the OGTT, which focuses on glucose metabolism, we also performed a nutritional challenge test that can impact a larger array of metabolic processes ${ }^{35}$, which has been previously used for the detection of nutritionally-induced metabolic differences ${ }^{26,36}$. A fasting-refeeding challenge was performed in indirect calorimetry after eight weeks of HFD feeding (PW 14), using the HDD as the ad libitum refeeding meal. The diet provides a rapid influx of glucose into the bloodstream, competing with protein and fat as additional substrates. The highly coordinated response of the organism to switch from predominantly 


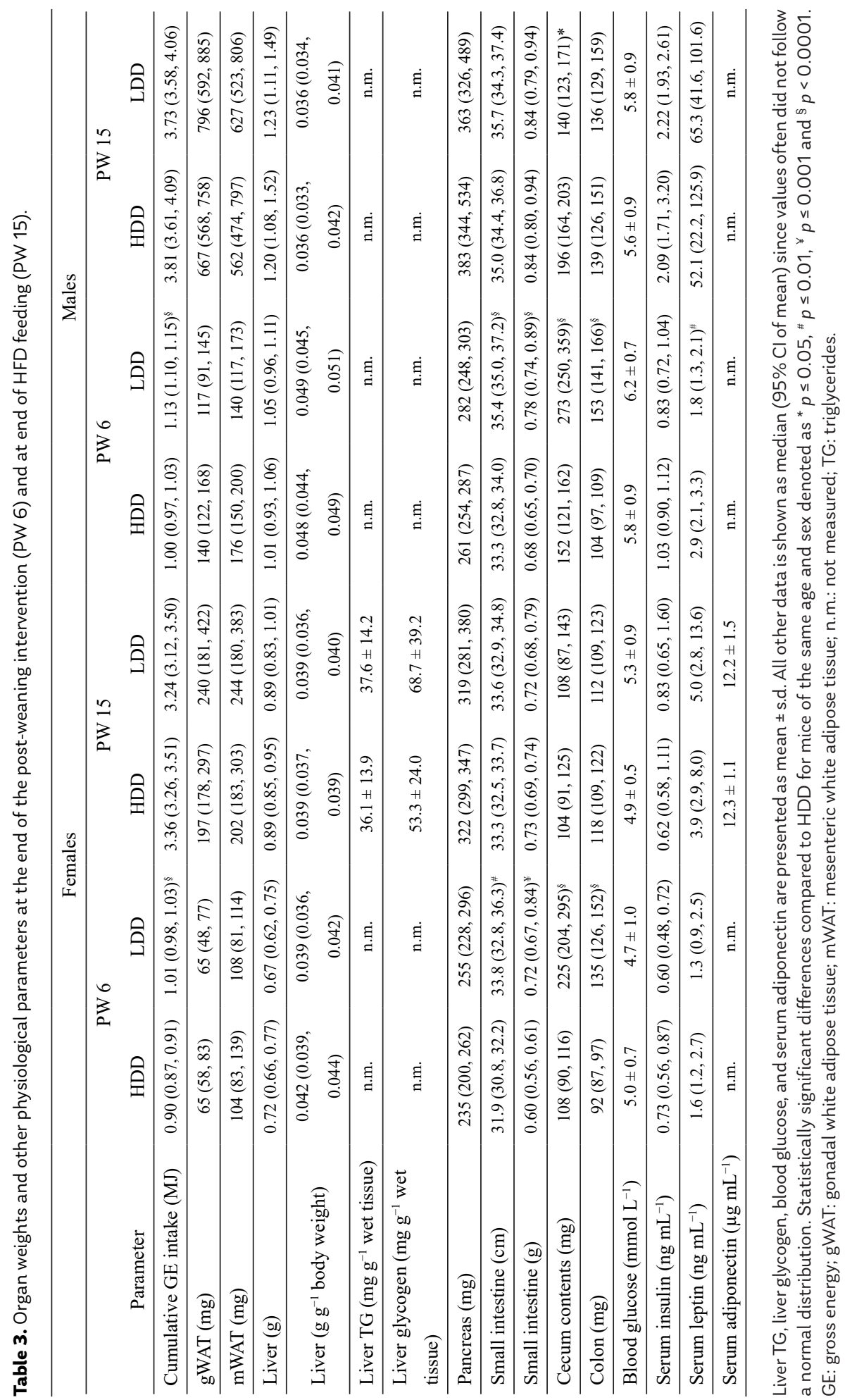



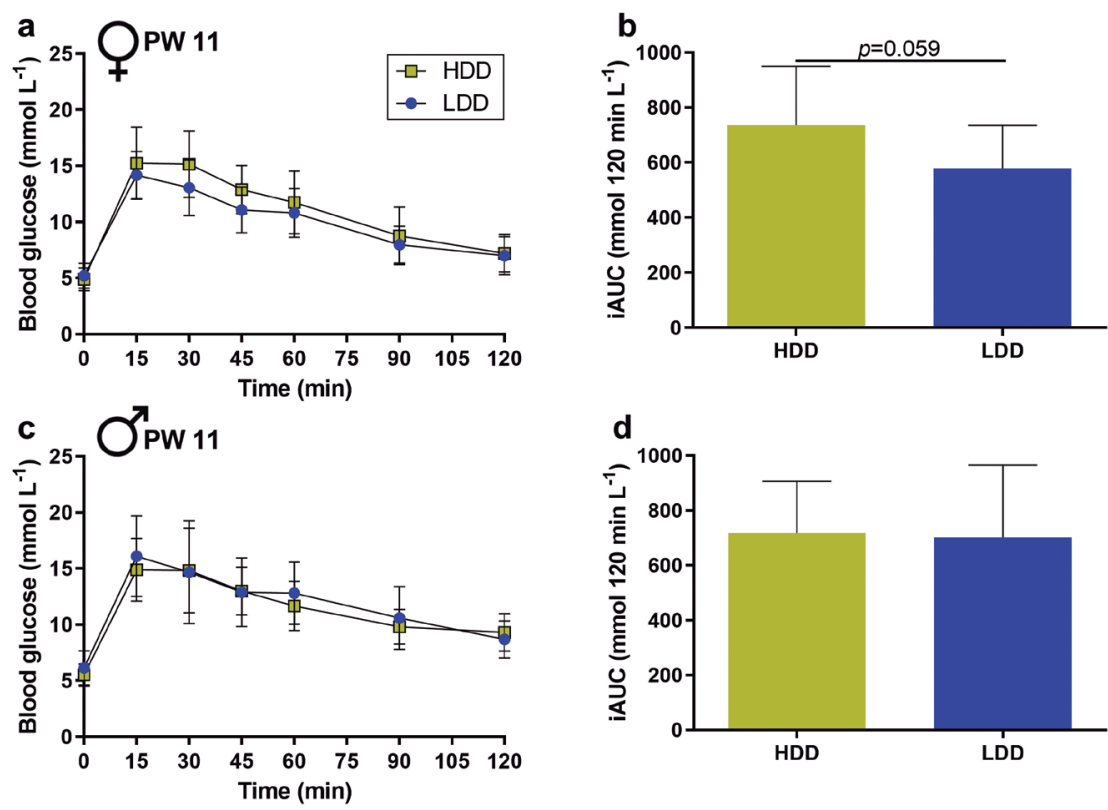

Figure 3. Glucose tolerance after five weeks of HFD feeding (PW 11). Plasma glucose concentrations measured directly before ( $\mathrm{O} \mathrm{min}$ ) and after oral administration of a glucose bolus ( $2 \mathrm{~g} \mathrm{~kg}^{-1} \mathrm{BW}$ ) in female (a, $n=12 \mathrm{for} \mathrm{HDD}$ and $n=11$ for LDD) and male (c, $n=11$ for HDD and $n=12$ for LDD) mice. Incremental area under the curve (iAUC) for blood glucose over the 120 min period for females $(\mathbf{b})$ and males (d). Data shown as mean \pm s.d.

fat oxidation (low RER) to glucose oxidation (high RER) was quantified as an indicator of metabolic flexibility ${ }^{27,36}$. Since the potential programming of glucose metabolism was hinted at only in females (Figure 3), we next focused primarily on females, presenting male data when available. The decline in RER after food restriction evolved in a virtually identical manner between mice fed HDD or LDD in the early post-weaning period, for both females and males, which also ensured that all of the mice were equally fasted before regaining access to food. Upon refeeding and particularly after the first hour of refeeding, the RER in females followed different trajectories, with a significant interaction between time and the post-weaning diet $(p<0.0001$, Figure $4 a)$. LDD females constantly had a numerically higher RER and reached a statistically significant higher RER at about six hours after access to food (Figure 4a). Similarly, the peak RER values that were reached within the refeeding period were higher in the LDD females (Figure $4 \mathrm{~b}$ ). The response in both the HDD and LDD males within three hours of refeeding was similar to that of the HDD females (Figure S2a), and the peak RER values that were reached upon refeeding were not significantly different between the male groups (Figure S2b). 
a

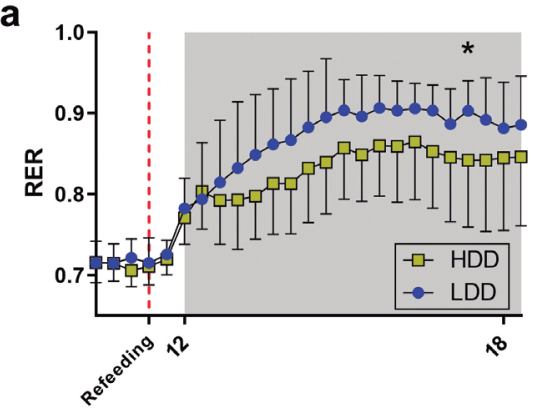

ZT

C

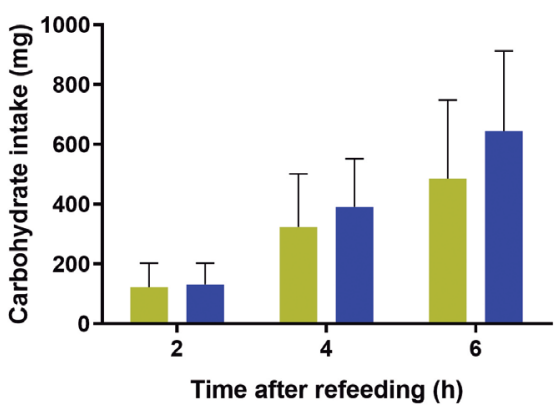

b

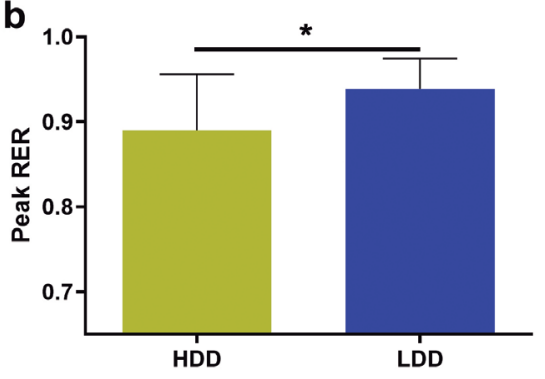

Figure 4. Metabolic flexibility of female mice after eight weeks of HFD feeding (PW 14). (a) RER evolution one hour before refeeding until seven hours upon ad libitum refeeding with a high carbohydrate diet (HDD). Statistical comparison was performed on all of the data points from the moment of food restriction (additional data points not shown to enhance visualization). (b) Mean peak RER values achieved within seven hours after refeeding. (c) Cumulative carbohydrate intake calculated from the automatic records of food intake after access to the refeeding diet. $n=13$ for HDD and $n=11$ for LDD. Data shown as mean \pm s.d. Statistical difference denoted as ${ }^{*} p \leq 0.05$.

To corroborate equal food intake during the challenge, we analyzed automatic food intake following access to food. There were no statistical differences in food intake between groups, neither in females (Figure 4c) nor in males (Figure S2c). Thus, our data points toward an improved capacity of LDD females to adapt fuel utilization to fuel availability, i.e., a better metabolic flexibility.

Direct and long-term effects on adipose tissue in females

While mean adipocyte size in gonadal white adipose tissue (gWAT) was not different between groups (PW 6: $35.4 \pm 4.5 \mu \mathrm{m}$ versus $31.6 \pm 4.0 \mu \mathrm{m}$; PW 15: $48.2 \pm 5.4 \mu \mathrm{m}$ versus $44.5 \pm$ $6.4 \mu \mathrm{m}$; mean \pm s.d., HDD and LDD respectively, representative pictures in Figure $5 \mathrm{a}-\mathrm{d}$ ), the distribution of adipocyte diameter in mice directly exposed to LDD was shifted toward smaller adipocytes compared to the HDD intervention (interaction between post-weaning diet and diameter bin: $p=0.0016$, Figure 5e). Nonetheless, the statistical differences in adipocyte size distribution disappeared after nine weeks of HFD feeding, although they were still bearing some visual resemblance to the distribution at PW 6 (Figure 5f). 
Adipocyte size has been linked to macrophage infiltration and inflammation ${ }^{29}$, which in turn has been linked to metabolic flexibility ${ }^{37}$. We therefore characterized macrophage and CLS abundance in gWAT depots. Both directly after the early post-weaning intervention and at the end of the HFD period, the gWAT pads of HDD mice harbored higher numbers of macrophages and CLS; however, only CLS in PW 6 and macrophages in PW 15 achieved statistical significance (Figure 5g,h). Interestingly, the mRNA levels in the gWAT pads in PW 15 of three macrophage markers, Lgals3, Ccl2, and S1008a-the latter a key gene associated with M1 macrophages-as well as two other genes linked to adipose tissue function (Irs2 and Fabp4), revealed no significant differences between groups (Figure S3). On the other hand, the histological data was consistent with the long-established association of macrophage abundance and CLS formation with adipocyte size in rodents ${ }^{29,38}$ (Figure 5i,j). Collectively, this data indicates that the type of starch had clear direct effects on adipose tissue morphology in females with associated differences in inflammation markers. These differences did not persist later in life.

\section{Discussion}

The direct differential effects of lowly-digestible versus highly-digestible-starch diets were seen in females for gWAT morphology and CLS abundance, and in males for whole body substrate metabolism and fat mass gain, with robust effects in the gut physiology in both sexes. Female mice that were subsequently fed a HFD into adulthood showed an improved capacity to adapt energy substrate utilization to substrate availability at the whole body level; however, this effect was not seen in males. This shows that metabolic flexibility in later life can be programmed by the type of starch in the early post-weaning diet in a sexdependent manner.

The direct metabolic effects by differences in starch digestibility can be due to two main factors: postprandial glycemia and gut microbiota. A highly-digestible starch will be more readily absorbed in the small intestine and stimulate insulin secretion more pronouncedly than a lowly-digestible starch, whereas a fraction of lowly-digestible starch will reach the cecum and colon and interact with gut microbiota. Both hyperglycemia and hyperinsulinemia could independently explain adipose tissue macrophage (ATM) homing and adipose tissue inflammation ${ }^{39}$. At the same time, gut microbiota is able to influence host health through multiple mechanisms ${ }^{40}$. Short-chain fatty acids (SCFA) derived from microbial fermentation have anti-inflammatory and other properties, directly inhibiting lipid storage via free fatty acid receptor 2 and indirectly increasing glucose uptake in adipocytes via the insulinreinforcing action of gut peptide $Y Y(P Y Y)^{41}$. The inhibition of fat storage and increased glucose disposal to adipose tissue would promote fatty acid utilization in other tissues, which is consistent with the lower RER seen in males consuming LDD. Moreover, as Zeevi et al. ${ }^{42}$ demonstrated, postprandial glycemic responses to the same meal depend partly on microbiota features. This, together with the observational evidence that a microbiota composition with a higher capacity for carbohydrate fermentation dampens weight loss in obese adults ${ }^{43}$, suggests a particularly important interaction between the two main factors that are involved in our study (postprandial glycemia and gut microbiota). Interestingly, our post-weaning LDD intervention led to a dramatically different fecal microbiota composition 


\section{a PW 6 HDD}

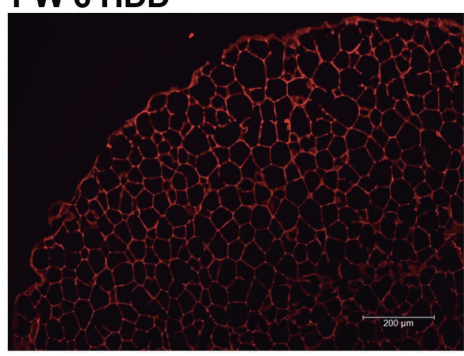

C PW 15 HDD

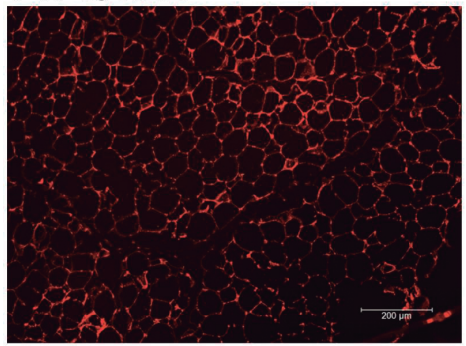

e

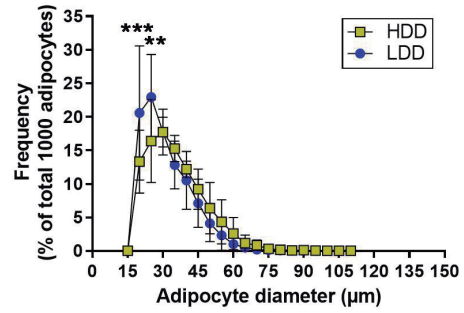

g

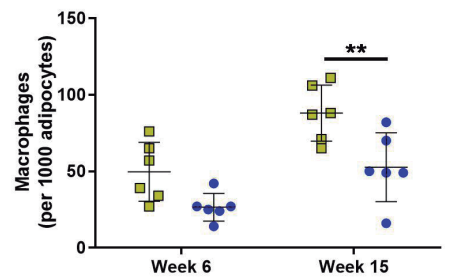

i

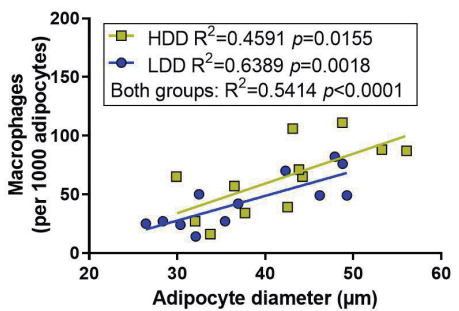

b PW 6 LDD

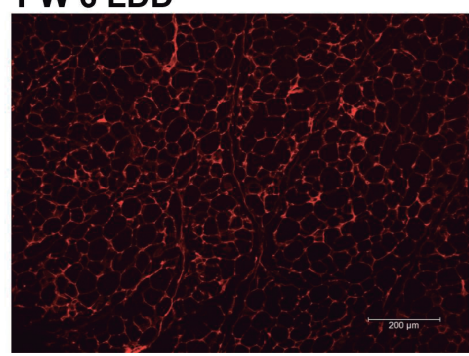

d PW 15 LDD

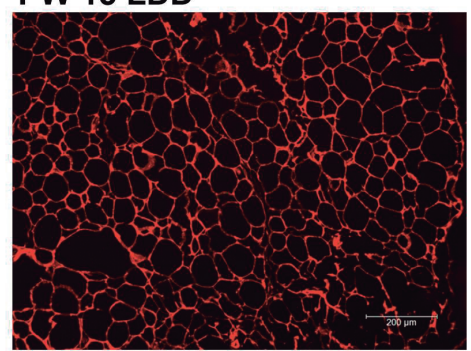

f

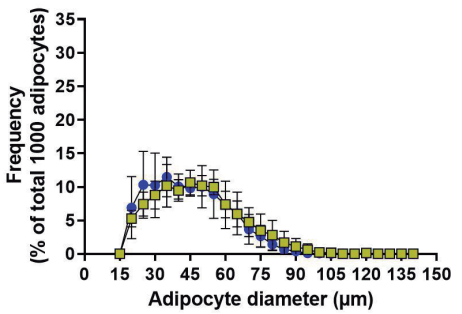

h

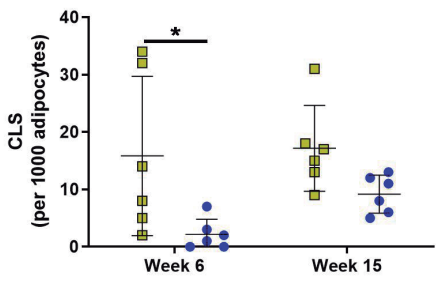

j

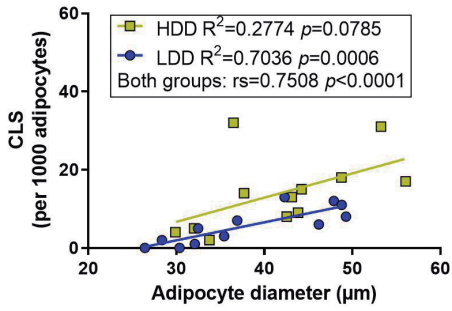




\section{CHAPTER 5}

Figure 5 (previous page). Adipose tissue histology, adipocyte size distribution, and macrophage infiltration markers in the gWAT of females at the end of the post-weaning intervention (PW 6) and after the HFD feeding period (PW 15). Representative histological pictures of gWAT using hematoxylin-eosin (HE) staining in HDD-fed (a) and LDD-fed mice (b) at the end of the post-weaning intervention (PW 6), and HDD-fed (c) and LDD-fed mice (d) after the HFD feeding period (PW 15). Photos were made using fluorescent microscopy. Distribution of adipocyte diameter ( $5 \mu \mathrm{m}$ bins) in gWAT pads collected in PW 6 (e) and PW 15 (f). Total count of macrophages (g) and crown-like structures (CLS, h) identified in the same areas using MAC-2 immunohistochemical staining. Correlation plots of adipocyte diameters with (i) macrophage and (j) CLS counts for all of the animals in each group. All of the coefficients were obtained with Pearson correlation except for the CLS of the combined HDD and LDD groups ( $r$ s = Spearman correlation coefficient). $n=12$ per dietary group, $n=$ six per age (PW 6 or PW 15). Data shown as mean \pm s.d. Statistical differences denoted as ${ }^{* *} p \leq 0.01$ and ${ }^{* * *} p \leq 0.001$.

in both sexes versus HDD-fed mice, with the increase in Parasutterella, Bacteroides, and Alloprevotella abundance after three weeks of LDD feeding strongly correlating only with $\mathrm{H}_{2}$ production, but not with body weight, fat mass, or food intake 9 . It is most likely that a combination of host and microbiota-mediated mechanisms explains the direct phenotypes of HDD and LDD mice. Although it has been demonstrated that some of the metabolic effects of resistant starches appear to be independent of the presence of a gut microbiota ${ }^{44}$, the starch in our LDD cannot quite be considered a resistant starch per $\mathrm{se}^{45}$.

The observed direct impact of starch digestibility on metabolic health in the present study is in accordance with recent findings on dietary Gl-induced effects in rodent models. Particularly, the increased fat mass in males fed a HDD is consistent with a recent metaanalysis of murine $\mathrm{Gl}$ studies showing that males benefit more from a low GI diet compared to females for several metabolic outcomes, including adiposity ${ }^{46}$. The lower RER observed in LDD males is also consistent with previous studies in rodents ${ }^{47}$. Our data do not show effects on BW and fasting glycemia, which may be due to the duration of dietary exposure ${ }^{46}$. A key observation in this study is the sex-specific effect of the type of starch on substrate utilization. This merits further investigation, especially because of the lack of experimental work in female models of nutrition, including starches of different digestibility ${ }^{46}$. It is unclear whether these differences are due to the effects of circulating sex hormones, which have been shown to be able to protect females from HFD-induced obesity and inflammation ${ }^{48}$, or alternatively, to developmental differences in metabolic regulation ${ }^{49}$.

Perhaps the strongest direct exposure effects were the enlargement of the lower intestinal tract in the LDD-fed female and male mice, supporting previous data on starches of low digestibility ${ }^{18,50}$. This enhanced growth of the intestine is in line with the trophic effects of SCFA ${ }^{51}$. Indeed, we observed increased SCFA in the cecum and colon upon LDD versus HDD feeding, as well as increased $\mathrm{H}_{2}$ production ${ }^{9}$. Yet another possibility is an effect of energy dilution due to the lower digestibility of the LDD. It has been demonstrated that mice invest in the growth of the stomach, ileum, cecum, and colon over three months of calorie restriction, at the same time preferentially utilizing WAT depots ${ }^{52}$. This investment in the alimentary tract was associated with a parallel increase in the assimilation efficiency of the diet ${ }^{52}$. In light of this evidence, it might be speculated that gut microbiota and host interact to maximize energy harvest in response to the lower nutrient availability in the LDD. 
The observed acute responses could have potential lifelong programming consequences. Indeed, after a nine-week period of the HFD, females fed the LDD showed a better metabolic flexibility in adulthood, supporting the potential of the early post-weaning diet to program metabolic health. In this sense, it is somewhat surprising that most of the other phenotypic parameters that were measured were similar, irrespective of the early post-weaning diet, and existing differences disappeared. Even the strong effects of the type of starch on the intestinal parameters seen in both sexes appeared to be absent later in life.

Sexual dimorphism in animal models of metabolic programming has been repeatedly observed, not only upon prenatal, but also postnatal exposure ${ }^{53}$. With regard to dietary carbohydrates, together with the study of Gugusheff et al. ${ }^{18}$, our work provides evidence that females are more susceptible to the long-term effects of particular types of starch on metabolic health. This is interesting given that, in line with our data shown here, the direct effects of starch digestibility are only seen in male rodents ${ }^{46}$. Surprisingly, males seemed largely unaffected over the long term, although we cannot exclude the possibility of programming other physiological outcomes. Moreover, sexual dimorphism in response to fasting has been only recently understood in great detail, with females actively promoting lipogenesis from amino acids, and males generally toning down anabolic pathways ${ }^{54}$, which could have major implications for metabolic flexibility. Additional studies would be needed to clarify whether females and males have indeed different developmental windows that are amenable to metabolic programming by starches.

On the whole, the metabolic consequences of early post-weaning starches were mild. This might be due to the physiologically relevant dietary levels of starches during the intervention, as well as the fat content during the period thereafter. Programming effects may have been more apparent using e.g., a 60 en\% fat HFD, which was required to reach maximal body weight and adiposity in a recent 'wild-type' mouse study with 29 diets varying in macronutrient proportions ${ }^{55}$. Alternatively, the mice we used are too healthy, which is supported by the OGTT responses. The usage of relevant disease models, such as mice with an impaired redox homeostasis (C57BL/6] mice from Jackson Laboratory in fact, as they have a mutated non-functional Nnt gene, in contrast to C57BL/6]RccHsd strain we used here, which has a functional Nnt gene) might provide opportunities. The strongest consequences of early post-weaning starches could also have been delayed, with aging being an important factor in the development of metabolic disease. Nevertheless, within nearly the same time span and study design, beneficial metabolic programming effects have been observed using specific lipids in the early post-weaning $\operatorname{diet}^{56,57}$.

On the question of what mechanisms could be responsible for the long-term effects of starches in the early post-weaning period, epigenetic processes are thought to underlie a considerable amount of programming phenomena, along with changes in tissue structure and accelerated cellular aging ${ }^{58,59}$. Neither we nor others ${ }^{17}$ have attempted to unravel epigenetic mechanisms specifically in the context of programming by carbohydrates, although there is a strong possibility that such mechanisms take place. For instance, key components of the machinery governing metabolic flexibility can be programmed by maternal nutrient and protein restriction, and are susceptible to epigenetic changes ${ }^{60-62}$. 
Moreover, some tissues retain plasticity to epigenetic modifications through early adulthood, as is the case for the brain and the colonic mucosa ${ }^{63,64}$, with butyrate-a SCFA-being able to cause epigenetic changes in the intestinal epithelium ${ }^{40}$. It is therefore conceivable that highly-digestible and lowly-digestible starches induce cellular biochemical changes that in turn cause epigenetic changes. Exactly which tissues are targeted is unclear.

We see value in placing the early post-weaning window that was chosen in this study within the current evolutionary paradigms of the Developmental Origins of Health and Disease $(\mathrm{DOHaD})$. One of the fundamental premises of the $\mathrm{DOHaD}$ framework is that phenotypic adaptations in response to early-life environmental cues, including nutrition, can be predictive of future environments ${ }^{65}$. When the anticipated environment does not match the actual conditions encountered later in life, such early phenotypic responses can become maladaptive and increase disease vulnerability. It is then conceivable that some of the programming effects that we report are not only determined by the post-weaning diet in itself, but also by earlier nutritional cues. In this way, the hormonal and metabolic environment promoted by carbohydrates during the suckling period could be very different to that encountered in the post-weaning period, depending on the carbohydrates introduced. There is at least one report arguing for this kind of carbohydrate mismatch ${ }^{18}$. Human breast milk is considered a low GI food ${ }^{66}$ \{Wright, 2015 \#804;Wright, 2015 \#804\}, and it also contains indigestible oligosaccharides that can influence the gut microbiome and SCFA profile ${ }^{67}$. Seen in this way, a lowly-digestible starch post-weaning diet could have produced a similar physiological environment as predicted during suckling, whereas a highly-digestible starch diet could fail to match the forecasted conditions and put the organism on course for disease.

\section{Conclusions}

Although the differences in the programmed adult phenotypes that we observed were subtle, our findings substantiate the notion that vulnerability to an obesogenic environment could partly depend on carbohydrate quality in early life. In line with the view that disease prevention must start with optimal nutrition early in life, our results need to be considered for the post-weaning diets as well as for products that target this period of growth and development.

\section{Author Contributions}

Conceptualisation, J.M.S.F.-C., L.M.S.B., A.O., J.K. and E.M.v.S.; Methodology, J.M.S.F.-C., H.J.M.S. and E.M.v.S.; Validation, J.M.S.F.-C. and E.M.v.S.; Formal Analysis, J.M.S.F.-C., and L.M.S.B.; Investigation, J.M.S.F.-C., L.M.S.B. and H.J.M.S.; Resources, H.J.M.S.; WritingOriginal Draft Preparation, J.M.S.F.-C.; Writing-Review \& Editing, J.M.S.F.-C., L.M.S.B., H.J.M.S., A.O., J.K. and E.M.v.S.; Visualisation, J.M.S.F.-C.; Supervision, J.K. and E.M.v.S.; Project Administration, J.M.S.F.-C., J.K. and E.M.v.S.; Funding Acquisition, A.O., J.K. and E.M.v.S. 


\section{Acknowledgments}

We would like to thank MSc students Yurike Widya-Inrumitha, Laura Dewitte, and Lini Sholihah and the personnel at the CARUS animal facility for their support. We also thank Inge van der Stelt for her excellent technical assistance. We thank Nils Billecke (Cargill R\&D Centre Europe, Belgium) for his contribution to data interpretation and thorough review of the manuscript. 


\section{CHAPTER 5}

\section{References}

Langley-Evans, S. C. Nutrition in early life and the programming of adult disease: a review. J. Hum. Nutr. Diet. 28 Suppl 1, 1-14 (2015).

Koletzko, B., Demmelmair, H., Grote, V., Prell, C. \& Weber, M. High protein intake in young children and increased weight gain and obesity risk. Am. J. Clin. Nutr. 103, 303-304 (2016).

Tang, M. Protein intake during the first two years of life and its association with growth and risk of overweight. Int. J. Environ. Res. Public Health 15, 1742 (2018).

Muhlhausler, B. S. \& Ailhaud, G. P. Omega-6 polyunsaturated fatty acids and the early origins of obesity. Curr. Opin. Endocrinol. Diabetes Obes. 20, 56-61 (2013).

Muhlhausler, B. S., Gibson, R. A. \& Makrides, M. Effect of long-chain polyunsaturated fatty acid supplementation during pregnancy or lactation on infant and child body composition: a systematic review. Am. J. Clin. Nutr. 92, 857-863 (2010).

Ludwig, D. S., Hu, F. B., Tappy, L. \& Brand-Miller, J. Dietary carbohydrates: role of quality and quantity in chronic disease. BMJ 361, k2340 (2018).

Schwingshackl, L., Hobl, L. P. \& Hoffmann, G. Effects of low glycaemic index/low glycaemic load vs. high glycaemic index/ high glycaemic load diets on overweight/obesity and associated risk factors in children and adolescents: a systematic review and meta-analysis. Nutr. J. 14, 87 (2015).

van Schothorst, E. M., Bunschoten, A., Schrauwen, P., Mensink, R. P. \& Keijer, J. Effects of a highfat, low- versus high-glycemic index diet: retardation of insulin resistance involves adipose tissue modulation. FASEB ]. 23, 1092-1101 (2009).

Fernández-Calleja, J. M. S. et al. Non-invasive continuous real-time in vivo analysis of microbial hydrogen production shows adaptation to fermentable carbohydrates in mice. Sci. Rep. 8, 15351 (2018).

Elia, M. \& Cummings, J. H. Physiological aspects of energy metabolism and gastrointestinal effects of carbohydrates. Eur. J. Clin. Nutr. 61 Suppl 1, S40-74 (2007).

Shaoul, R., Tiosano, D. \& Hochberg, Z. Evo-devo of child growth: the role of weaning in the transition from infancy to childhood. Crit. Rev. Food Sci. Nutr. 56, 887-895 (2016).

Wopereis, H., Oozeer, R., Knipping, K., Belzer, C. \& Knol, J. The first thousand days - intestinal microbiology of early life: establishing a symbiosis. Pediatr. Allergy Immunol. 25, 428-438 (2014).

Fewtrell, M. et al. Complementary feeding: a position paper by the European Society for Paediatric Gastroenterology, Hepatology, and Nutrition (ESPGHAN) Committee on Nutrition. J. Pediatr. Gastroenterol. Nutr. 64, 119-132 (2017).

Woo Baidal, J. A. et al. Risk factors for childhood obesity in the first 1,000 days: a systematic review. Am. J. Prev. Med. 50, 761-779 (2016).

Food and Agriculture Organization of the United Nations. Carbohydrates in Human Nutrition: Report of a Joint FAO/WHO Expert Consultation, Rome, 14-18 April 1997. (FAO, Rome, 1998).

Penfold, N. C. \& Ozanne, S. E. Developmental programming by maternal obesity in 2015: Outcomes, mechanisms, and potential interventions. Horm. Behav. 76, 143-152 (2015).

Patel, M. S. \& Srinivasan, M. Metabolic programming in the immediate postnatal life. Ann. Nutr. Metab. 58 Suppl 2, 18-28 (2011).

Gugusheff, J. et al. The effect of maternal and post-weaning low and high glycaemic index diets on glucose tolerance, fat deposition and hepatic function in rat offspring. J. Deu. Orig. Health Dis. 7, 320329 (2015).

Oosting, A. et al. Effect of dietary lipid structure in early postnatal life on mouse adipose tissue development and function in adulthood. Br. J. Nutr. 111, 215-226 (2014). 
Minana-Solis Mdel, C. \& Escobar, C. Post-weaning protein malnutrition in the rat produces short and long term metabolic impairment, in contrast to earlier and later periods. Int. J. Biol. Sci. 4, 422-432 (2008).

He, Y. H. et al. Postweaning low-calcium diet promotes later-life obesity induced by a high-fat diet. J. Nutr. Biochem. 23, 1238-1244 (2012).

Shahkhalili, Y., Mace, K., Moulin, J., Zbinden, I. \& Acheson, K. J. The fat:carbohydrate energy ratio of the weaning diet programs later susceptibility to obesity in male sprague dawley rats. J. Nutr. 141, 81-86 (2011).

Bouwman, L. M. S. et al. No adverse programming by post-weaning dietary fructose of body weight, adiposity, glucose tolerance, or metabolic flexibility. Mol. Nutr. Food Res. 62, 1700315 (2018).

Hoevenaars, F. P. et al. BIOCLAIMS standard diet (BIOsd): a reference diet for nutritional physiology. Genes Nutr. 7, 399-404 (2012).

Duivenvoorde, L. P. et al. A difference in fatty acid composition of isocaloric high-fat diets alters metabolic flexibility in male C57BL/6]OlaHsd Mice. PLoS One 10, e0128515 (2015).

Muoio, D. M. Metabolic inflexibility: when mitochondrial indecision leads to metabolic gridlock. Cell 159, 1253-1262 (2014).

Krisman, C. R. A method for the colorimetric estimation of glycogen with iodine. Anal. Biochem. 4 17-23 (1962).

Cinti, S. et al. Adipocyte death defines macrophage localization and function in adipose tissue of obese mice and humans. J. Lipid Res. 46, 2347-2355 (2005).

Carpenter, A. E. et al. CellProfiler: image analysis software for identifying and quantifying cell phenotypes. Genome Biol. 7, R100 (2006).

CellProfiler, http://forum.cellprofiler.org/t/adipocyte-h-e-cell-profiler-pipeline/1460 (2012)

Van Schothorst, E. M. et al. Adipose gene expression patterns of weight gain suggest counteracting steroid hormone synthesis. Obes. Res. 13, 1031-1041 (2005).

Fischbach, M. A. \& Sonnenburg, J. L. Eating for two: how metabolism establishes interspecies interactions in the gut. Cell Host Microbe 10, 336-347 (2011).

van Ommen, B., Keijer, J., Heil, S. G. \& Kaput, J. Challenging homeostasis to define biomarkers for nutrition related health. Mol. Nutr. Food Res. 53, 795-804 (2009).

Stroeve, J. H., van Wietmarschen, H., Kremer, B. H., van Ommen, B. \& Wopereis, S. Phenotypic flexibility as a measure of health: the optimal nutritional stress response test. Genes Nutr. 10, 459 (2015).

Horakova, O. et al. Preservation of metabolic flexibility in skeletal muscle by a combined use of n-3 PUFA and rosiglitazone in dietary obese mice. PLoS One 7, e43764 (2012).

Sparks, L. M. et al. Relation of adipose tissue to metabolic flexibility. Diabetes Res. Clin. Pract.

83, 3243 (2009).

Weisberg, S. P. et al. Obesity is associated with macrophage accumulation in adipose tissue. J. Clin. Invest. 112, 1796-1808 (2003).

Boutens, L. \& Stienstra, R. Adipose tissue macrophages: going off track during obesity. Diabetologia 59, 879-894 (2016).

Tilg, H. \& Moschen, A. R. Food, immunity, and the microbiome. Gastroenterology 148, 1107-1119 (2015).

den Besten, G. et al. The role of short-chain fatty acids in the interplay between diet, gut microbiota, and host energy metabolism. J. Lipid. Res. 54, 2325-2340 (2013). (2015). 


\section{CHAPTER 5}

43 Muñiz Pedrogo, D. A. et al. Gut microbial carbohydrate metabolism hinders weight loss in overweight adults undergoing lifestyle intervention with a volumetric diet. Mayo Clin. Proc. 93, 1104-1110 (2018)

44 Bindels, L. B. et al. Resistant starch can improve insulin sensitivity independently of the gut microbiota. Microbiome 5, 12 (2017).

45 Yang, X. et al. Resistant starch regulates gut microbiota: structure, biochemistry and cell signalling. Cell. Physiol. Biochem. 42, 306-318 (2017).

Campbell, G. J., Senior, A. M. \& Bell-Anderson, K. S. Metabolic effects of high glycaemic index diets: a systematic review and meta-analysis of feeding studies in mice and rats. Nutrients 9, 646 (2017).

Higgins, J. A. Resistant starch and energy balance: impact on weight loss and maintenance. Crit. Reu. Food Sci. Nutr. 54, 1158-1166 (2014).

Grove, K. L., Fried, S. K., Greenberg, A. S., Xiao, X. Q. \& Clegg, D. J. A microarray analysis of sexual dimorphism of adipose tissues in high-fat-diet-induced obese mice. Int. J. Obes. 34, 989-1000 (2010).

Karastergiou, K. \& Fried, S. K. Cellular mechanisms driving sex differences in adipose tissue biology and body shape in humans and mouse models. Adv. Exp. Med. Biol. 1043, 29-51 (2017).

Charrier, J. A. et al. High fat diet partially attenuates fermentation responses in rats fed resistant starch from high-amylose maize. Obesity 21, 2350-2355 (2013).

Topping, D. L. \& Clifton, P. M. Short-chain fatty acids and human colonic function: roles of resistant starch and nonstarch polysaccharides. Physiol. Reu. 81, 1031-1064 (2001).

Mitchell, S. E. et al. The effects of graded levels of calorie restriction: I. impact of short term calorie and protein restriction on body composition in the C57BL/6 mouse. Oncotarget 6, 15902-15930 (2015)

Aiken, C. E. \& Ozanne, S. E. Sex differences in developmental programming models. Reproduction 145 , R1-13 (2013).

Della Torre, S. et al. Short-term fasting reveals amino acid metabolism as a major sex-discriminating factor in the liver. Cell Metab. 28, 256-267 e255 (2018).

$\mathrm{Hu}$, S. et al. Dietary fat, but not protein or carbohydrate, regulates energy intake and causes adiposity in mice. Cell Metab. 28, 415-431 e414 (2018).

Oosting, A. et al. Size and phospholipid coating of lipid droplets in the diet of young mice modify body fat accumulation in adulthood. Pediatr. Res. 72, 362-369 (2012).

Baars, A. et al. Milk fat globule membrane coating of large lipid droplets in the diet of young mice prevents body fat accumulation in adulthood. Br. J. Nutr. 115, 1930-1937 (2016).

Vickers, M. H. Early life nutrition, epigenetics and programming of later life disease. Nutrients $\mathbf{6}$, 2165-2178 (2014).

Reichetzeder, C., Dwi Putra, S. E., Li, J. \& Hocher, B. Developmental origins of disease - crisis precipitates change. Cell. Physiol. Biochem. 39, 919-938 (2016).

Miles, J. L., Huber, K., Thompson, N. M., Davison, M. \& Breier, B. H. Moderate daily exercise activates metabolic flexibility to prevent prenatally induced obesity. Endocrinology 150,179-186 (2009).

Holness, M. J., Zariwala, G., Walker, C. G. \& Sugden, M. C. Adipocyte pyruvate dehydrogenase kinase 4 expression is associated with augmented PPARgamma upregulation in early-life programming of later obesity. FEBS Open Bio 2, 32-36 (2012). energy resources and requirements in health and disease. Endocr. Reu. 39, 489-517 (2018). 
CHAPTER 5

64 Kellermayer, R. et al. Epigenetic maturation in colonic mucosa continues beyond infancy in mice. Hum. Mol. Genet. 19, 2168-2176 (2010).

65 Gluckman, P. D., Hanson, M. A. \& Buklijas, T. A conceptual framework for the developmental origins of health and disease. J. Deu. Orig. Health Dis. 1, 6-18 (2010).

Wright, C. J., Atkinson, F. S., Ramalingam, N., Buyken, A. E. \& Brand-Miller, J. C. Effects of human milk and formula on postprandial glycaemia and insulinaemia. Eur. J. Clin. Nutr. 69, 939-943 (2015).

67 Le Huerou-Luron, I., Blat, S. \& Boudry, G. Breast- v. formula-feeding: impacts on the digestive tract and immediate and long-term health effects. Nutr. Res. Rev. 23, 23-36 (2010). 


\section{Supplementary Information}
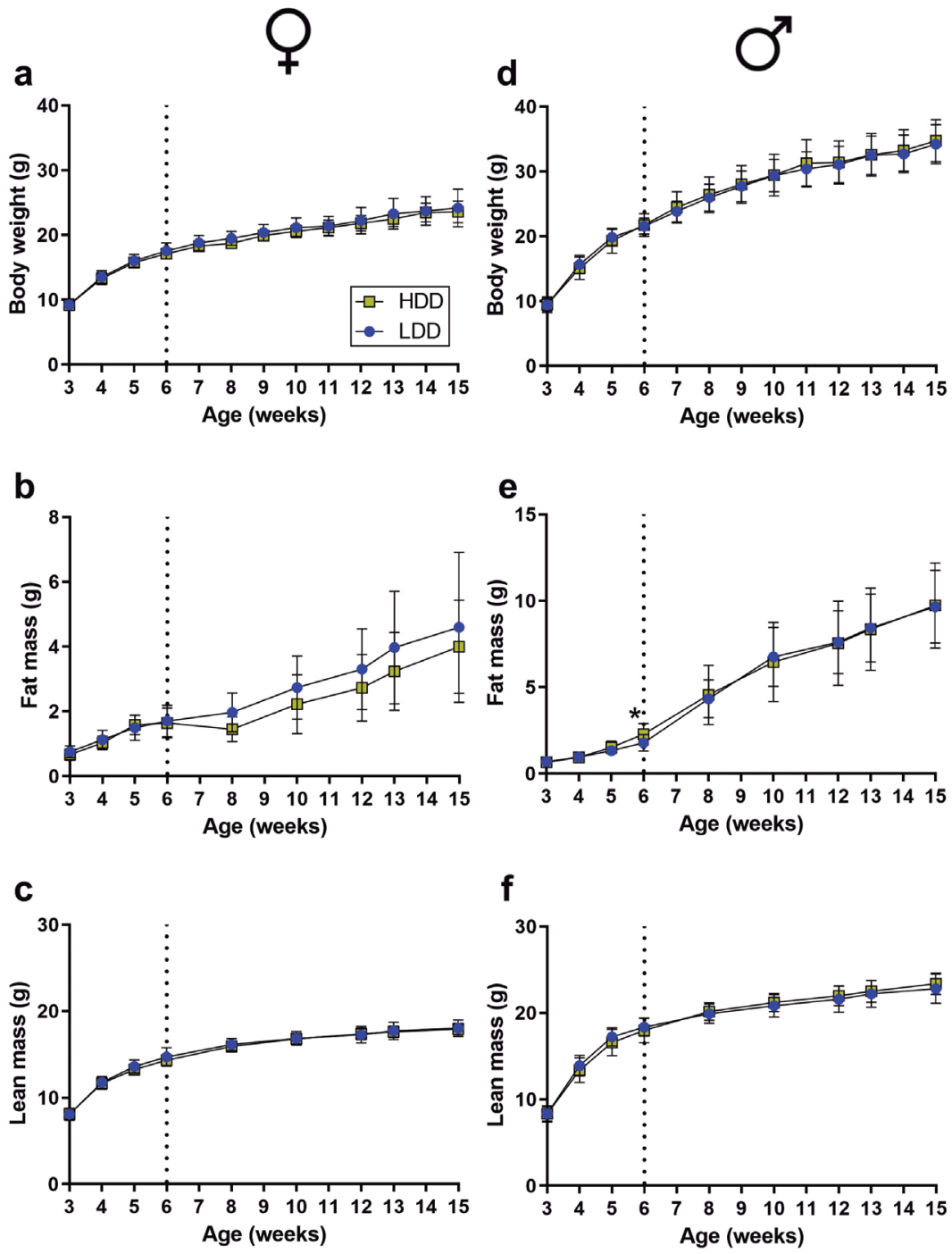

Figure S1. Body weight and body composition development. (a) Body weight (BW), (b) fat mass (FM), and (c) lean mass (LM) of females from weaning at postnatal week (PW) 3 until the end of the study $(n=24$ per group from PW 3 through 6; $n=12$ per group from PW 7 through 15). (d) BW, (e) FM, and (f) LM of males from weaning at PW 3 until the end of the study ( $n=24$ for HDD and $n=23$ for LDD from PW 3 through $6 ; n=12$ per group from PW 7 through 15). To test for nutritional programming effects independently of initial differences at the start of HFD feeding, data corresponding to the starch intervention period ( $n=23-24$ per group and sex) and the HFD period ( $n=12$ per group and sex) were analysed separately by repeated measurements two-way ANOVA with Bonferroni's post hoc test. In both sexes and periods, both HDD and LDD gained BW, FM, and LM over time $(p<0.0001)$. No difference was seen between the two dietary groups, except for the increase in FM in males during the intervention period $(F=4.8, p=0.0344)$. However, males fed LDD us HDD during the intervention period gained $B W(F=2.9$, $p=0.0355)$ and $F M(F=8.5, p<0.0001)$ at a lower rate, reaching a significant difference in FM at PW $6(p<0.0001)$. Data shown as mean \pm s.d. The dotted line indicates the start of HFD feeding. 
a

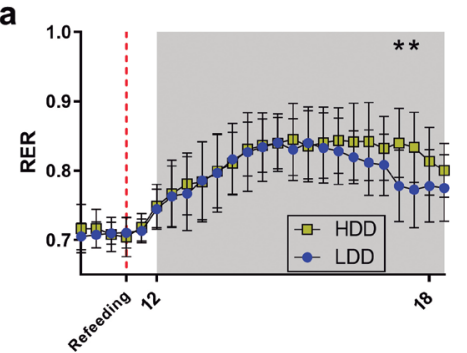

ZT

C

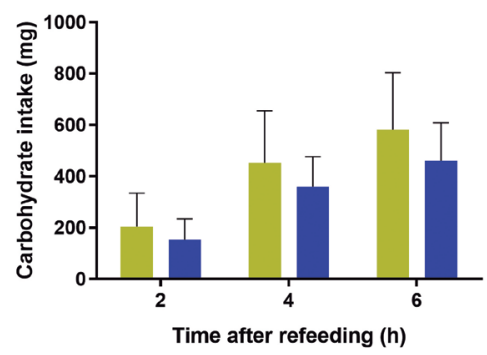

b

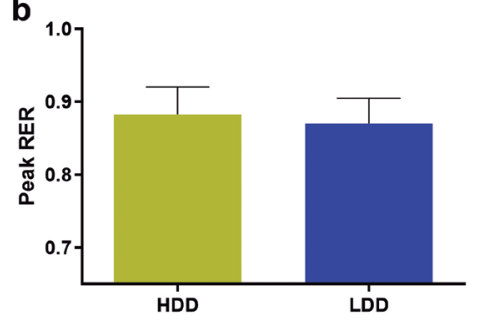

Figure S2. Metabolic flexibility of male mice after 8 weeks of HFD feeding (PW 14). (a) RER evolution $1 \mathrm{~h}$ before refeeding until $7 \mathrm{~h}$ upon ad libitum refeeding with a high carbohydrate diet (HDD). Statistical comparison was performed on all data points from the moment of food restriction (additional data points not shown to enhance visualisation). There was a significant interaction of time $\mathrm{x}$ post-weaning $\operatorname{diet}(p=0.0163)$. (b) Mean peak RER values achieved within $7 \mathrm{~h}$ after refeeding. (c) Cumulative carbohydrate intake calculated from automatic records of food intake after access to refeeding diet. $n=12$ per group. Data shown as mean \pm s.d. Statistical differences denoted as ${ }^{*} p \leq 0.001$.

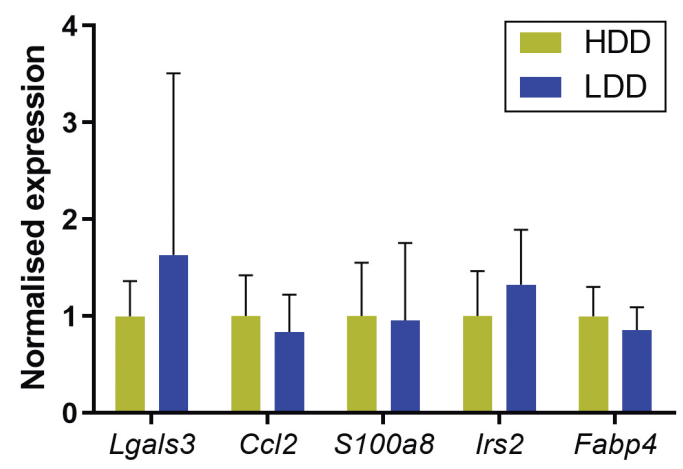

Figure S3. Gene expression in gWAT of females after the HFD feeding period (PW 15). $n=11$ for HDD and $n=10$ for LDD. Values are given relative to the mean normalised gene expression of the HDD group for each gene and plotted as mean \pm s.d. Lgals3: lectin, galactose binding, soluble 3; Ccl2: chemokine (C-C motif) ligand 2; S100a8: S100 calcium binding protein A8 (calgranulin A); Irs2: insulin receptor substrate 2; Fabp4: fatty acid binding protein 4 , adipocyte. 
CHAPTER 5

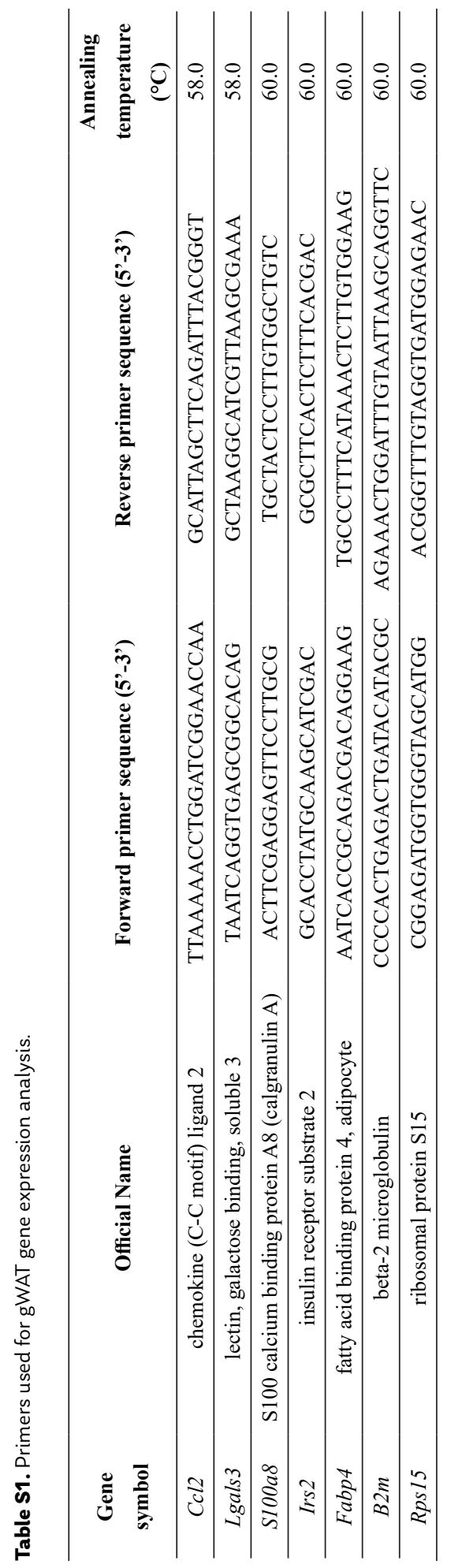




\section{Chapter}

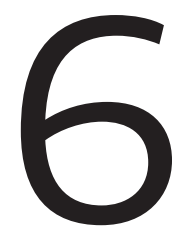

General Discussion 

The major aims of this thesis were 1) to develop and show the added value of an extended mouse metabolic phenotyping tool based on indirect calorimetry $(\ln C a)$ for the real-time study of microbiota activity and the oxidation of exogenous us endogenous substrates; and 2) to apply this tool to study the direct effects and metabolic programming effects of starches consumed during the early post-weaning period.

In Chapter 2, we developed an extended $\ln \mathrm{Ca}$ (elnCa) system by incorporating hydrogen $\left(\mathrm{H}_{2}\right)$ and methane $\left(\mathrm{CH}_{4}\right)$ sensors. Gut microbial fermentation, measured as $\mathrm{H}_{2}$ production, was higher in mice fed a lowly digestible starch diet (LDD) compared to a highly digestible starch diet (HDD) and exhibited a circadian pattern. Short and long-term consumption of LDD compared to HDD produced consistent changes in faecal bacterial taxa, and several of these bacteria were positively or negatively correlated to $\mathrm{H}_{2}$ production. $\mathrm{CH}_{4}$ was not produced at detectable concentrations on any diet, consistent with the absence of faecal methanogens. We further extended the elnCa system with ${ }^{13} \mathrm{CO}_{2}$ and ${ }^{12} \mathrm{CO}_{2}$ sensors in Chapter 3, allowing us to obtain real-time ${ }^{13} \mathrm{CO}_{2}$ enrichment data. The elnCa system detected circadian patterns in ${ }^{13} \mathrm{CO}_{2}$ enrichment corresponding to the natural isotopic enrichments of dietary ingredients and endogenous fuels. In addition, by enabling the separate quantification of the rates of exogenous us endogenous fuels, elnCa detected an impairment of dietary fat oxidation in obese mice. The value of elnCa for metabolic phenotyping studies was further shown in Chapter $\mathbf{4}$, where we tested the direct effects of a 3 wk intervention with LDD us HDD in weanling females and males. Daily $\mathrm{H}_{2}$ production and the cumulative oxidation of a ${ }^{13} \mathrm{C}$-labelled starch bolus were higher in LDD mice, but only LDD females showed significantly faster starch-derived glucose oxidation kinetics within 45 min after receiving the bolus compared to HDD females. Finally, both the direct and metabolic programming effects of post-weaning LDD us HDD were tested in Chapter 5. In both sexes, $\mathrm{H}_{2}$ production was confirmed to be directly increased by LDD. Females on LDD had a higher frequency of small adipocytes and abundance of crown-like structures (CLS) in gonadal white adipose tissue (gWAT) at the end of the post-weaning diet compared to females on HDD, but LDD males only had an increased fat mass (FM) compared to HDD males. After a period on a high-fat diet (HFD), adult females previously exposed to LDD had a better metabolic flexibility and lower macrophage infiltration in gWAT compared to HDD. The postweaning starches did not show a metabolic programming effect in males.

Until the studies completed in this work, there were no suitable methods to continuously study mouse EE, gut microbiota activity, and exogenous us endogenous substrate oxidation rates in a single phenotyping test. Also, there were few studies comparing the direct metabolic effects of lowly us highly digestible starches in both females and males, and there were no studies of the long-term metabolic consequences of starches consumed in early life. In this chapter, I will discuss what the above findings could mean for future technological developments in rodent studies, the potential implications of starch digestibility in early life for human health, and recommendations to approach animal experiments on nutritional programming. 


\section{Extended Indirect Calorimetry (elnCa)}

Hydrogen $\left(\mathrm{H}_{2}\right)$ and methane $\left(\mathrm{CH}_{4}\right)$ sensors

We measured $\mathrm{H}_{2}$ as an indicator of gut microbial activity. As we have seen in Chapter 2, $\mathrm{H}_{2}$ production depended on the digestibility of the diet, followed a circadian pattern, and was strongly linked to specific changes in faecal microbiota taxa. But what does $\mathrm{H}_{2}$ really indicate? Carbohydrates consumed but not assimilated by the host can be anaerobically catabolised in a series of redox reactions that provide energy to microbes. This process is called fermentation and results in the production of $\mathrm{H}_{2}, \mathrm{CO}_{2}$, and SCFAs. The $\mathrm{H}_{2}$ generated by fermentative bacteria must be kept below a certain threshold, because a high $\mathrm{H}_{2}$ partial pressure will inhibit bacterial growth and slow the rate of fermentation ${ }^{1}$. Disposing of $\mathrm{H}_{2}$ can be accomplished in two ways. One way is by excretion of $\mathrm{H}_{2}$ in flatus and breath (after absorption into the circulation), the latter known to account for up to $21 \%$ of the $\mathrm{H}_{2}$ produced in the human intestine ${ }^{2}$. The other, by utilisation of $\mathrm{H}_{2}$ by hydrogenotrophic bacteria to obtain energy, mainly through oxidation of $\mathrm{H}_{2}$ into $\mathrm{H}_{2} \mathrm{~S}$ and $\mathrm{CH}_{4}$ by sulfate reducing bacteria and methanogens, respectively. This implies that high levels of $\mathrm{H}_{2}$ detected in the air in mouse cages reflect the disposal of excessive gas and an abundance of fermentative substrates. Thus, $\mathrm{H}_{2}$ as an indicator of availability of substrates for fermentation explains the higher $\mathrm{H}_{2}$ levels in LDD- us HDD-fed mice in Chapters 2, 4, and 5. A pulsed supply of nutrients to the gut microbiota also explains the circadian patterns in $\mathrm{H}_{2}$ levels and their similarity to food intake (FI) events observed in Chapter 2. In fact, other fermentation markers, i.e. caecal SCFAs, also show a circadian pattern ${ }^{4}$. The circadian pattern of fermentation is likely driven solely by $\mathrm{Fl}$, as we found no clear association between locomotor activity and $\mathrm{H}_{2}$ production. However, the relationship between microbial activity and $\mathrm{FI}$ may be bi-directional. An emerging model of host-microbiota appetite regulation proposes that gut microbial activity stimulates intestinal hormone release and satiety pathways in the host, and that these pathways are less stimulated as microbial populations decrease (as would be the case in the postabsorptive state) ${ }^{5}$.

By including $\mathrm{H}_{2}$ analysis in real time, elnCa offers an attractive tool to study the flexibility of the microbiota to adapt to substrate availability in the short and the long term. The kinetics of faecal microbial community and caecal SCFA changes in response to fermentable carbohydrates have only been studied at intervals no shorter than one day ${ }^{6-8}$. These studies have thought us that the complete adaptation to increased availability of fermentable substrates is a long and nuanced process. The earliest changes in microbial community structures have been seen after 1-4 $d$ in humans, and some of these changes are transient while others are sustained ${ }^{7,9}$. The extent and types of caecal fermentation during this process also vary, with lactic acid fermentations predominantly occurring within the first $5 \mathrm{~d}$ of a LDD in rats, and SCFA production being increasingly favoured during the following weeks ${ }^{8}$. Within $5 \mathrm{~d}$, the early changes in microbial fermentation are concomitant to host anatomical and physiological adaptations like caecum enlargement and increased nitrogen fluxes ${ }^{8}$. In Chapter 2, we have indeed observed that half of the faecal bacterial taxa increased by 3 wk on LDD were also increased after $4.5 \mathrm{~d}$ of LDD feeding, and that the small and large intestines were enlarged in LDD us HDD mice 
after $3 \mathrm{wk}$ (regrettably we were unable to measure this parameter also at $4.5 \mathrm{~d}$ because it required sacrificing an additional group of mice). Nocturnal $\mathrm{H}_{2}$ production was already increased by $700 \%$ in LDD mice by $4.5 \mathrm{~d}$ after switching from the chow diet, compared to the $\sim 850 \%$ increase in $\mathrm{H}_{2}$ output in mice adapted to LDD us HDD for 3 wk, consistent with the known progressive increase in microbial fermentation mentioned above. Here, the added value of $\mathrm{H}_{2}$ measurements gave insight into the very early stages ( $<1 \mathrm{~d}$ ) of adaptation to fermentable carbohydrates non-invasively, as we were able to observe that cumulative $\mathrm{H}_{2}$ production was already doubled during the first $12 \mathrm{~h}$ after switching mice from chow to LDD us HDD. This shows that the gut microbiota are more responsive to substrate availability in the very short term, much shorter than previously known, and aligns with the transit time of 6-8 $\mathrm{h}$ reported in mice ${ }^{10}$.

Besides $\mathrm{H}_{2}$, other fermentation gases like $\mathrm{CH}_{4}$ should be taken into account to get a more complete picture of the gut microbial activity occurring in vivo, which as we show here, can be done without additional animal discomfort. This could be especially useful if $\mathrm{H}_{2}$ excretion equals or approaches zero, when all $\mathrm{H}_{2}$ produced could be disposed of by hydrogenotrophs. In this study, for the diets and mouse strains used and metabolic states (fasted us fed) investigated we could not detect $\mathrm{CH}_{4}$ production (Chapters 2-5), and this was consistent with the absence of methanogenic archaea in faecal samples (Chapter 2). $\mathrm{CH}_{4}$ excretion has been measured in rats and mice ${ }^{11-13}$, and methanogens have been detected in mouse caecum ${ }^{14}$ and faeces ${ }^{15}$. Likely, the $\mathrm{H}_{2}$ produced by fermentative bacteria in fasted and HDD mice in this study was utilised by sulfate reducing bacteria instead. Especially when the supply of dietary carbohydrates is limited, microbes also ferment dietary proteins and endogenous nutrients (e.g. glycoproteins secreted by goblet cells, or mucins), leading to the production of $\mathrm{H}_{2}$, branched chain fatty acids (BCFAs), phenols, and $\mathrm{H}_{2} \mathrm{~S}$ (ref. 16). Indeed, the reliance of the gut microbiota on protein and mucin glycans was suggested in Chapter 2 by the higher abundance of caecal isovaleric acid (a BCFA) and faecal Desulfovibrio (a sulfate reducer) in HDD us LDD mice. Thus, incorporating a $\mathrm{H}_{2} \mathrm{~S}$ sensor could offer an additional and non-invasive way to probe into these hydrogenotrophic processes. Currently, most of our understanding of the interactions between $\mathrm{H}_{2}$-producing fermentative bacteria and $\mathrm{H}_{2}$-consuming hydrogenotrophs is theoretical and based on in vitro evidence ${ }^{17}$. A rodent elnCa system enabling $\mathrm{H}_{2}, \mathrm{CH}_{4}$, and $\mathrm{H}_{2} \mathrm{~S}$ analysis in real-time could be an excellent next step to study how microbial communities compete for $\mathrm{H}_{2}$ in the gut and how this influences host health.

Monitoring host metabolism and microbial activity simultaneously promises to complement the phenotypic assessment of physiological and pathophysiological conditions. Fermentation gases have been implicated in health and disease states. For instance, $\mathrm{H}_{2}$ is related to intestinal bloating and discomfort in humans, but at the same time $\mathrm{H}_{2}$ could be of therapeutic value because of the antioxidative, antiapoptotic, and anti-inflammatory activities of exogenous $\mathrm{H}_{2}$ as demonstrated in both in vitro and in vivo models of reperfusion injury, liver inflammation, drug-induced nephrotoxicity, allergy, Parkinson's disease, and colitis $^{18}$. There is also some evidence for an LDL cholesterol-lowering and improved glucose tolerance effect of supplemental $\mathrm{H}_{2}$ in drinking water in humans at risk of metabolic syndrome ${ }^{19,20}$. Thus, it is possible that the availability of endogenous $\mathrm{H}_{2}$ to the host may be beneficial for metabolic health. Furthermore, increased utilisation of fermentation $\mathrm{H}_{2}$ 
by sulfate reducing bacteria leads to the production of cytotoxic and pro-inflammatory metabolites like $\mathrm{p}$-Cresol and $\mathrm{H}_{2} \mathrm{~S}$, which have been linked to colorectal cancer ${ }^{21,22} . \mathrm{H}_{2} \mathrm{~S}$ has also other physiological effects as a gasotransmitter similar to nitric oxide (NO) and carbon monoxide $(\mathrm{CO})^{23}$, underscoring the potential of microbial $\mathrm{H}_{2}$ metabolism to influence the overall health status of the host. Fermentation gases could also impact the host's energy balance. On the one hand, a positive energy balance could be favoured by utilisation of $\mathrm{H}_{2}$ by hydrogenotrophs, which increases the efficiency of fermentation and yields more SCFAs to the host that can in turn oxidise them to obtain energy ${ }^{1}$. In addition, mitochondrial $\mathrm{H}_{2} \mathrm{~S}$ oxidation at low concentrations can be a source of electrons for the electron transport chain, indicating that $\mathrm{H}_{2} \mathrm{~S}$ can also provide energy to the host ${ }^{24}$. On the other hand, a negative energy balance could be promoted by $\mathrm{H}_{2} \mathrm{~S}$ too, as this gas has been shown to modulate thermogenesis in brown adipose tissue, allowing the dissipation of energy as heat ${ }^{25}$. Some of the energy content of the diet is also lost to fermentation gases. In ruminants, who rely heavily on microbial fermentation, $\mathrm{CH}_{4}$ excretion is a crucial component of energy metabolism ${ }^{26}$. The loss of energy to $\mathrm{H}_{2}$ and $\mathrm{CH}_{4}$ produced by humans consuming non-digestible polysaccharides has been considered negligible, representing $0.2 \%$ of daily $\mathrm{EE}^{27}$. This has not been directly determined in rodents, but energy lost to $\mathrm{H}_{2}$ has also been deemed unimportant in one study of the effects of lowly digestible starch on energy balance in rats $^{28}$. We have seen that the fraction of daily energy expenditure (EE) lost to $\mathrm{H}_{2}$ in LDD-fed mice is $0.02 \%$ (Chapter 2). Assuming a constant metabolic rate of $44.2 \mathrm{~kJ} \mathrm{~d}^{-1}\left(11 \mathrm{kcal} \mathrm{d}^{-1}\right)$, this would represent a loss of $7.4 \mathrm{~kJ}$ (2 kcal, or about $220 \mathrm{mg}$ of fat tissue; for comparison, we have seen up to $8 \mathrm{~g}$ of fat tissue in middle-aged chow-fed males) over the entire adulthood and postreproductive stages of a C57BL/6] male mouse (841 d, ref. 29). Although fermentation gases did not lead to sizeable differences in EE in our mice, it is possible that larger amounts of energy are lost as excreted $\mathrm{H}_{2}$ or $\mathrm{CH}_{4}$ at higher quantities or with other types of fermentable carbohydrates in the diet. Moreover, behavioural factors may decrease the digestibility of the food and increase the availability of substrates to the gut microbiota. In Chapter 2, after a period of food restriction, 2 out of 4 mice produced more than double the volume of $\mathrm{H}_{2}$ per $\mathrm{g}$ of chow consumed compared to their production before food restriction. Hydrogen excretion in these mice peaked at $1.7 \mathrm{~h}$ after regaining access to food. This is considerably earlier than the $3 \mathrm{~h}$ it would take food to reach the caecum, based on a mouse study of transit time using a radiolabelled activated charcoal bolus ${ }^{10}$. Possibly, those mice in Chapter $\mathbf{2}$ could have ingested food very rapidly, limiting chewing and resulting in larger particles in the chyme that may be difficult to digest, thus forming substrates for fermentation. Chow contains whole grains, and it is known that starches in whole grains are entrapped in a non-digestible matrix that can become physically accessible if this matrix is broken down mechanically ${ }^{30}$. Alternatively, gastric emptying could have been accelerated by increased ghrelin levels ${ }^{31}$. The possibility that fasting and then refeeding leads to a less efficient nutrient assimilation should be considered in mouse and human studies, as fasting-refeeding is the basis of various metabolic phenotyping tests ${ }^{32}$. Thus, accurately quantifying the excretion of fermentation gases in tandem with host metabolic gas exchange introduces a new dimension to the phenotyping power of rodent InCa and adds microbial activity to the standard components of EE like basal metabolic rate and physical activity. 
${ }^{13} \mathrm{CO}_{2}$ and ${ }^{12} \mathrm{CO}_{2}$ sensors

The combination of ${ }^{13} \mathrm{CO}_{2}$ enrichment analysis and respirometry is rare in rodent studies, while there are many studies using either technique separately. Why is it important to integrate both techniques? Enabling ${ }^{13} \mathrm{CO}_{2}$ enrichment analysis in tandem with $\mathrm{O}_{2}$ and $\mathrm{CO}_{2}$ analysis yields quantitative data: not only do we know the proportion of ${ }^{13} \mathrm{CO}_{2}$ content in mouse cages, but also the rate at which $\mathrm{CO}_{2}$ is being produced. Early since stable isotopes were used in human research, the technique has been combined with quantitative respirometry in humans, and in 1976 the first study applied both techniques to calculate the amount of a dose of glucose that became oxidised over $7 \mathrm{~h}$ (ref. 33). Only decades later, this combination started to be implemented in non-model species ${ }^{34}$. In mice, however, the only two instances where quantitative ${ }^{13} \mathrm{CO}_{2}$ analysis has been achieved are the studies of Ishihara et. $a^{35}$ and $\mathrm{McCue}$ et. $a^{36}$. Ishihara et al. developed a combination of mass spectrometry and $\mathrm{O}_{2}$ and $\mathrm{CO}_{2}$ analysers ${ }^{37}$ and soon applied it to dissect the oxidation of exogenous us endogenous glucose and fat in diabetic mice fed casein- or soy proteinbased diets ${ }^{35}$. This system seemed promising because of its online analysis mode and high temporal resolution ( $<1 \mathrm{~h}$, although this is not consistently reported in their studies). Surprisingly, the investigators have reported the use of the system only once more, when they used ${ }^{13} \mathrm{CO}_{2}$ from ${ }^{13} \mathrm{C}$ acetate as a marker of gastric emptying (thus, only using ${ }^{13} \mathrm{CO}_{2}$ enrichment data, not respirometry data $)^{38}$, nor have others constructed a similar system. By painstakingly collecting air samples from metabolic cages every hour, McCue et al. obtained quantitative data of endogenous carbohydrate, lipid, and protein oxidation in mice raised on ${ }^{13} \mathrm{C}$ labelled tracers and deprived of food for $72 \mathrm{~h}$. In this study, substrate partitioning could not be reliably interpreted from RER data only because of the high intraand interindividual variation in RER values and the changes accompanying acid-base balance occurring during prolonged fasting ${ }^{36}$. Because the body carbohydrate, lipid, and protein stores were respectively labelled by ${ }^{13} \mathrm{C}$ glucose, ${ }^{13} \mathrm{C}$ palmitate, or ${ }^{13} \mathrm{C}$ leucine, the authors were able to calculate well-defined shifts in fuel oxidation in the progression of fasting. Carbohydrate oxidation was rapidly minimised but briefly increased around $12 \mathrm{~h}$ after food deprivation, lipids became the most important fuel from $2 \mathrm{~h}$ onwards, and protein was initially spared ( $8 \%$ EE between 8 and $12 \mathrm{~h}$ ), but then became increasingly important $(24 \% \text { at } 72 \mathrm{~h})^{36}$. These results showed that the three phases of fuel use during fasting and starvation (oxidation of carbohydrates, then lipids, then proteins) are not as straightforward as typically stated. The combination of ${ }^{13} \mathrm{CO}_{2}$ analysis and respirometry was key to these conclusions: absolute protein oxidation was constant after the initial protein sparing stage, but its relative contribution to EE increased as fasting progressed ${ }^{36}$. Moreover, the duration and magnitude of the protein-sparing phase could not have been identified by relying on urinary $\mathrm{N}$ content, because those measurements are not possible at such short intervals $(1 \mathrm{~h})$. The study in Chapter $\mathbf{4}$ also illustrates how both isotopic enrichment and respirometry data must be interpreted together. Our question was whether the oxidation kinetics of a single starch bolus fed to young mice that had been on an LDD us HDD for 3 wk would be different and sex-dependent. Early upon administration of the starch bolus, ${ }^{13} \mathrm{CO}_{2}$ enrichment tended to increase faster in LDD us HDD males (Chapter 4 Figure S2a, 2-way RM ANOVA, time $\times$ diet interaction $P=0.095$; Bonferroni multiple comparisons $P<0.05$ at $t=20$ and $40 \mathrm{~min}$ ). When ${ }^{13} \mathrm{CO}_{2}$ enrichment data and the rate of 


\section{CHAPTER 6}

total $\mathrm{CO}_{2}$ production $\left(\mathrm{VCO}_{2}\right)$ were used to calculate the true oxidation rates of the starch bolus, an effect of LDD us HDD was not apparent anymore (Chapter 4 Figure 3a, time $\times$ diet interaction $P=0.999)$. After receiving the bolus, HDD mice had a burst in activity (data not shown) and increased their metabolic rate more than LDD males (Chapter 4 Figure S3a). In other words, the proportion of ${ }^{13} \mathrm{CO}_{2}$ to ${ }^{12} \mathrm{CO}_{2}$ was indeed higher for LDD males, but the absolute quantity of ${ }^{13} \mathrm{CO}_{2}$ produced (reflecting the quantity of starch oxidised) was similar, offsetting the difference in the oxidation kinetics between LDD and HDD males. In Chapter $\mathbf{4}$ we did not include behavioural aspects a priori (other than FI), but this result might give us a lead to investigate, for instance, whether the sudden activity burst of HDD mice could be due to lower feelings of satiety. Incidentally, rodents fed lowly digestible starch have persistent and elevated circulating GLP-1 (glucagon-like peptide 1, a satiety hormone), even when they tend to consume more food than those fed highly digestible $\operatorname{starch}^{39}$.

I propose more emphasis should be put on quantifying oxidation data in mouse experiments that use isotopic tracers. There are excellent examples of well-designed metabolic tracer studies that could have been refined by including quantitative respirometry. Friedrich et al. showed that epigallocatechin gallate (EGCG), a bioactive compound in green tea, increased exogenous fat oxidation in $\mathrm{BL} / 6 \mathrm{~N}$ mice ${ }^{40}$. The authors had to rely on ${ }^{13} \mathrm{CO}_{2}$ enrichment data only (measured in air samples from mice placed inside a $140 \mathrm{ml}$ syringe) to conclude that EGCG promotes the oxidation of a dose of ${ }^{13} \mathrm{C}$ palmitate. This data was backed by their previous study using InCa, where oral administration of EGCG before the dark phase (DP) decreased RER in ad libitum fed NZB mice, and no effects of EGCG on EE were apparent ${ }^{41}$. Because of the different mouse strain used by Friedrich et al., it cannot be confidently excluded that the postprandial metabolic rate of EGCG-treated mice is higher than control mice, leading to a higher proportion of ${ }^{13} \mathrm{CO}_{2}$ inside the syringe. This could imply that EGCG might not specifically enhance the oxidation of recently ingested fat. Actually, the data supports that dietary fat is not absorbed as efficiently in EGCC mice, as shown by the increased ${ }^{13} \mathrm{C}$ enrichment in faeces and decreased expression of fatty acid transporters in intestinal mucosa ${ }^{40}$. A combined isotopic enrichment-InCa approach could have provided stronger evidence in a single experiment, integrating the true oxidation rates of ingested fat with metabolic rate and meal events. The use of this combined approach is a rare find in literature, and previous attempts at it have been either expensive or laborious. Our elnCa provides a way forward.

The data in Chapters $\mathbf{3}$ and $\mathbf{4}$ suggested that part of the ${ }^{13} \mathrm{C}$ label orally administered was likely sequestered in TCA cycle metabolites, as we have seen in the delayed exogenous substrate us total substrate oxidation curves. This effect is known to occur in humans ${ }^{42}$ and is corrected for in metabolic studies with ingested fatty acid tracers by means of a dietary acetate recovery factor $(\mathrm{dARF})^{43}$. Including a dARF in exogenous substrate oxidation calculations could impact the conclusions of metabolic tracer studies, since the dARF is lower for overweight us lean humans ${ }^{44}$. There might be a similar relationship between adiposity and label sequestration in mice. In Chapter $\mathbf{4}$, the negative correlation between body FM and the oxidation of palmitate ingested in a mixed meal became less strong after applying the dARF of humans according to their FM. We assumed human dARFs would be 
valid for mice because, at present, the data necessary to calculate a dARF for mice is not available. The correlation in Chapter $\mathbf{4}$ hinted at an impairment in nutrient sensing in the obese state, and the results fit the hypothesis that the capacity to store fat is enhanced in obesity. This hypothesis has been around for some time and is supported by radioactive tracer studies in the rat, with increased adipose tissue LPL activity and altered hypothalamic sensing of fatty acids as potential mechanisms ${ }^{45,46}$. The relation between nutrient sensing and obesity may be causal in this model ${ }^{47}$, but this has been recently challenged in humans. In an ongoing cohort of self-reported obesity-prone and obesity-resistant humans, there were no differences in their ability to couple fat intake to fat oxidation upon consumption of a labelled mixed meal after acute overfeeding or eucaloric feeding ${ }^{48}$. If an inverse relationship between the dARF and adiposity is also present in rodents, failing to apply a correction in the exogenous substrate oxidation formulas could overestimate dietary fatty acid oxidation in obese mice and rats, explaining the discrepancy in these models. At the time we obtained the ethical approval for the experiments compiled in this thesis, we were only aware that the ${ }^{13} \mathrm{C}$ label could be temporarily sequestered in the bicarbonate pool, and that this factor was negligible due to the short half-life of ${ }^{13} \mathrm{C}$ enriched bicarbonate in mice $(\sim 15 \mathrm{~min})^{49}$, but a new mouse study with orally administered ${ }^{13} \mathrm{C}$ acetate could be performed in elnCa at low cost and with minimal invasiveness to determine a mousespecific dARF.

Widespread application of elnCa can result in greater insights into metabolism. Functional metabolic challenges, like fasting and refeeding challenge tests, can be refined with the use of elnCa. We have seen in Chapter $\mathbf{3}$ that exogenous glucose oxidation was not part of the metabolically inflexible phenotype of obese mice, but rather total glucose oxidation and exogenous fat oxidation were impaired. Impaired total glucose oxidation is indicative for impaired insulin action, which operates on whole body level, not on exogenous glucose only. This only became apparent after complementing the standard RER-based metabolic flexibility test with ${ }^{13} \mathrm{CO}_{2}$ enrichment analysis. Furthermore, interpreting the oxidation of a two-fuel mixture (glucose and fatty acids) based on RER can sometimes be misleading. In certain situations, RER values can drop below 0.704 , the oft-quoted value indicative of maximal net fat oxidation with respect to net glucose oxidation. We have observed RER $<0.704$ in fasted animals in Chapters $\mathbf{3}$ and $\mathbf{4}$, and in previous experiments in our laboratory ${ }^{50}$. While low RER values can result from erroneous $\mathrm{O}_{2}$ and $\mathrm{CO}_{2}$ measurements ${ }^{51}$, these values were likely physiological as we optimized measurements by regular calibration and sufficient sample intervals between measurements of mouse and reference cages. During prolonged fasting, gluconeogenesis from protein and ketogenesis in excess of ketone body oxidation can become important and can lower RER to values below 0.704 , overestimating fat oxidation and underestimating glucose utilisation ${ }^{52,53}$. The assessment of perturbations of substrate use by refeeding challenges is complicated by the fact that, due to the limited gastric volumes of mice, refeeding is ethically done with small amounts of food. Because RER measurements are not very sensitive to small changes in substrate flux $^{54}$, this would in turn produce relatively small and transient increases in RER. In our hands, a glucose dose of $\sim 70 \mathrm{mg}\left(2 \mathrm{~g} \mathrm{~kg}^{-1}\right.$ body weight, BW) produced maximal RER responses of about 0.80 (ref. 50), and a $40 \mathrm{mg}$ starch bolus elicited similar maximal RER responses of 0.81 (Chapter 4 ). Others have found higher RER increments using a larger 


\section{CHAPTER 6}

glucose dose: $225 \mathrm{mg}$ per mouse ( $8.8 \mathrm{~g} \mathrm{~kg} \mathrm{BW}^{-1}$ ) produced an RER peak of 0.91 (ref. 55). This dose however, is far higher than commonly used 1 or $2 \mathrm{~g} \mathrm{~kg}^{-1} \mathrm{BW}$ (ref. 56) and may not be physiological. In Chapter 4, RER fell after 45 min on administration of the starch bolus, often below 0.704 . Under these conditions, net glucose oxidation would have been interpreted as zero, but the ongoing oxidation of the starch bolus was quantifiable thanks to the ${ }^{13} \mathrm{CO}_{2}$ and ${ }^{12} \mathrm{CO}_{2}$ analysers. Although less common, pathophysiological conditions can also invalidate the standard assumptions of substrate use based on RER. Shinozaki et al. have shown that changes in $\mathrm{VO}_{2}$ are not linked to changes in $\mathrm{VCO}_{2}$ in the post-cardiac arrest rat model ${ }^{57}$. After resuscitation of the rat and $\mathrm{O}_{2}$ inhalation, the cellular utilization of $\mathrm{O}_{2}$ increased disproportionally to the production of $\mathrm{CO}_{2}$ after resuscitation, producing RER values lower than 0.7 (with inhalation of $30 \% \mathrm{O}_{2}$ ) or even below 0.6 (with inhalation of $100 \% \mathrm{O}_{2}$ ), the latter value outside the physiological range ${ }^{57}$. In this study, loading the body carbohydrate, fat, or protein pools with ${ }^{13} \mathrm{C}$ labelled substrates and measuring ${ }^{13} \mathrm{CO}_{2}$ during the post-cardiac arrest period would have allowed for a correct interpretation of substrate utilisation.

The above examples of elnCa as a deep phenotyping tool for rodents opens new possibilities for future extensions. Incorporating a sensor for volatile organic compounds (VOCs) would be of great interest. VOCs are increasingly used to study metabolic responses in humans and have diagnostic potential ${ }^{58,59}$. In this sense, excreted acetone could be measured and used to quantitatively correct for ketogenesis in the substrate oxidation formulas, going beyond the oxidative fate of nutrients and into biosynthetic processes. Other biomarkers could be further exploited or validated. Isoprene has been proposed as an indicator of the rates of cholesterol production ${ }^{58}$, although it is also likely to respond to physiological challenges like exercise $^{60}$. More broadly, other compounds like pentane (a marker of lipid peroxidation and reperfusion injury ${ }^{61}$ ) and toluene (a marker of lung cancer ${ }^{62}$ ) could be informative in mouse models of human pathophysiology. For these and the sensors described in this thesis, future technological challenges lie ahead. It will be important to establish, for instance, if the responsiveness of the ${ }^{13} \mathrm{CO}_{2}$ and ${ }^{12} \mathrm{CO}_{2}$ sensors to natural isotopic enrichments, as seen in the mice fed wheat-based and maize-based ingredients in Chapter 3, can also be used to quantify substrate oxidation rates. Although our experiments with non-labelled diets were not designed to quantify substrate oxidation rates, we concluded that the sensors are at least sensitive to the very small differences in ${ }^{13} \mathrm{CO}_{2}$ enrichment usually detected by mass spectroscopy. Other challenges will be to make affordable the incorporation of a sensor for every mouse cage to allow second-to-second resolution without compromising accuracy (already possible for the conventional $\mathrm{O}_{2}$ and $\mathrm{CO}_{2}$ sensors ${ }^{63}$ ), and to properly filter out other possible sources of gases and VOCs, like bedding and food pellets.

\section{Conclusions}

Our studies demonstrate how InCa can be extended with additional gas sensors and applied directly to areas of metabolic research where rodent models are essential. By simultaneously providing quantitative information on energy expenditure, microbiota activity, and oxidation of exogenous us endogenous substrates, elnCa maximised the value of a single in vivo phenotypic assessment. Decidedly, getting more data out a 
single phenotyping assessment contributes to the ethical use (or the three Rs: reduction, refinement, and replacement) of animals in experimental research.

\section{Metabolic Consequences of Consuming Lowly us Highly Digestible Starches}

In parallel with the technological innovations discussed in the previous section, the research questions addressed in this thesis were initially conceived in the context of metabolic programming by lowly us highly digestible starches. The mouse model used allowed for the distinction between the direct effects of starches during the post-weaning period and their programming effects onto adult life, i.e. long after the exposure of starches of different digestibility. This means that, while Chapters $\mathbf{4}$ and $\mathbf{5}$ primarily focussed on the direct and programming effects of starches, respectively, there is considerable overlap with the study designs across all Chapters. Therefore, data from other Chapters will be integrated when appropriate. The reader is first presented with a discussion on the findings regarding the direct effects of LDD us HDD. This will aid in the interpretation of the metabolic programming outcomes, discussed later. I will then briefly reflect on the translational value of our findings.

\section{Sex-dependant direct effects of weaning starch on metabolism}

As we have seen in Chapters $\mathbf{4}$ and $\mathbf{5}$, the metabolic outcomes in mice directly exposed to LDD were generally beneficial compared to mice exposed to HDD and consistent with what is reported in the meta-analysis of Campbell et al. for low glycaemic index (GI) diets in rodent studies, which were mostly designed as lowly digestible starch diets based on amylose content ${ }^{64}$. In our studies, however, fasting glucose and insulin concentrations were not affected by starch digestibility. The $3 \mathrm{wk}$ duration of the starch interventions was short compared to the median duration of $10 \mathrm{wk}$ in the studies analysed by Campbell et al, likely explaining why these static metabolic parameters (as opposed to functional ones, like those measured by challenge tests) were unchanged in our studies. However, we also observed that the oxidative disposal of a starch bolus was enhanced in LDD mice, demonstrating that carbohydrate metabolism was affected by starch digestibility in the short term. Although we found this effect to be stronger in females, the improved starch oxidation in males seem to contradict the observations of Isken et al., who found no effects of starch digestibility on the oxidation of an oral glucose bolus in adult male mice after a $3 \mathrm{wk}$ intervention $^{65}$. This may be related to the age of the mice at the start of the experiment, but may also be due to the form of glucose used to test carbohydrate oxidation, since we used starch instead of pure glucose. A more extensive oxidation of the starch bolus could be a consequence of enhanced digestion. We have investigated this possibility, but amylase levels in the small intestine were not different in LDD compared to HDD mice. The trend towards higher deposition of the ${ }^{13} \mathrm{C}$ label in the LDD liver was also not sufficient to explain the differences in starch oxidation. The preferential oxidation of starch-derived glucose in LDD mice could result from a decreased glucose partitioning to de novo lipogenesis. Others have shown that consumption of lowly digestible starch led to smaller adipocytes in rat epididymal adipose tissue and higher insulin-stimulated ${ }^{14} \mathrm{C}$ glucose oxidation ex vivo ${ }^{66}$. 
In accordance, we found a higher frequency of small adipocytes in gWAT of LDD us HDD females in Chapter 5. However, FM was lower in LDD us HDD males (a bigger effect compared to females) despite their more similar exogenous glucose oxidation (a smaller effect compared to females), which could be the primary reason for smaller adipocytes. More research is needed to understand how and why consuming lowly us highly digestible starches causes the same starch molecule to be handled differently.

A recurrent observation in this thesis was the sexually dimorphic response to starch digestibility. As an explicit question from the outset, this was reassuring, yet the mechanisms behind the phenomenon remain elusive. Inherent differences between females and males, namely gonadal hormones and the sex chromosomes, may shape the metabolic responses to nutritional factors and lead to sexually dimorphic phenotypes. For lack of a mechanistic explanation, I will explore a few potential avenues for further research.

There are a number of sex dimorphisms in body composition, insulin sensitivity, relative blood volume and distribution, temperature and pain perception, and depot-specific metabolism, cellularity, and growth of adipose tissue ${ }^{67-69}$. Some of our findings, like the more complete oxidation of starch in males us females (Chapter 4), may be easily explained by the higher lean mass and lower adiposity of males. A contribution of sexual maturity may also be involved. At least for exercising humans, it is known that exogenous glucose oxidation depends on pubertal stage, with children being more "oxidative" than adults $^{70}$, and that this may be related to a different capacity to load or tap into endogenous glycogen stores ${ }^{71}$. The onset of puberty occurs later in male than in female mice ${ }^{72}$. In this sense, a more oxidative phenotype of sexually immature males would be consistent with the overall more extensive oxidation of the starch bolus in males us females in Chapter 4.

The tougher question here is, exactly how could any of the above factors account for the discrepant female-male HDD and LDD phenotypes? Further targeted experimentation is needed before we can propose a detailed mechanism for the sex-specific metabolic response to starches of different digestibility, but the gut microbiome might be involved. Sex is known to influence microbiota communities in mice, and these in turn influence sex hormone levels and host metabolism ${ }^{73}$. Although we found that sex did not significantly contribute to faecal microbiota composition after 3 wk on LDD us HDD (Chapter 2), it is still possible that the metabolic pathways of female us male microbiota were differently activated as we did not analyse the metatranscriptome. This is conceivable because, despite the profound sequencing depth used for our microbial taxonomy analysis (i.e. genus level), there are structural variants in the genomes of otherwise identical microbial strains that could confer them different functions ${ }^{74}$. Even if the composition and metabolic activity of the gut microbiota were identical, gut microbial metabolites can be differently handled by females and males. By way of example, gut microbes can convert dietary choline to trimethylamine, which in turn is metabolised into trimethylamine-N-oxide (TMNO) by the hepatic enzyme flavin monooxygenase 3 (ref. 75). This hepatic enzyme is overexpressed (about 100 fold higher) in females us males, leading to higher plasma levels of TMNO in females, and at the same time TMNO explained $11 \%$ of the variation in atherosclerotic lesions in a panel of 100 inbred mouse strains ${ }^{76}$. This opens the possibility that gut microbial metabolites 
commonly derived from LDD us HDD in both sexes had a different impact on the host's metabolic phenotype depending on sex.

Potentially interesting here is the sexual differentiation of liver metabolism, which starts before puberty. In the female liver, but not the male liver, the response to short-term fasting is to catabolise amino acids to synthesise lipids (to keep up building energy stores for the completion of ovulation) and glucose (both to maintain euglycaemia and to provide NADPH for lipogenesis, through the pentose phosphate pathway ${ }^{77}$. This may be especially relevant for nutrient challenge tests conducted in the fasted state when there is additional supply of nutrients derived from fermentation (i.e. SCFAs). We have seen larger caecum contents in fasted LDD females and males, suggesting that SCFA may be available to the host (Chapter 4). Accordingly, fasting RER was higher in LDD ( 0.73) us HDD ( 0.70) males, suggesting that LDD males oxidised the available SCFA, leading to a higher RER (Chapter 4). In females, fasting RER was lower than in males and not different between LDD and HDD females ( 0.68; Chapter 4). The default switch of the liver to gluconeogenesis from amino acids during short fasting may partly explain a RER lower than 0.7 in females, but not the similar RER between HDD and LDD females. Speculatively, SCFA could have been used by LDD females as an additional substrate for lipogenesis instead of being oxidised.

It is thus not very clear what makes females respond differently to starch digestibility than males. Whatever the mechanism, it may also induce different phenotypes in the long run. As we will see shortly, there was indeed sexual dimorphism in the metabolic programming effects of starches. Sex dimorphisms in metabolic programming are well known, but a common drawback is that if these effects are measured too early they may reflect pubertal status rather than primarily sex differences ${ }^{78}$. However, we have measured effects both before complete sexual maturation and in mature animals, allowing us to interpret the sexdependent metabolic programming effects more cleanly.

Metabolic programming effects of lowly us highly digestible starches

The metabolic programming effects we observed were modest and only evident in females. In Chapter 5, LDD us HDD females tended to have a better oral glucose tolerance when assessed 6 wk after introduction of the HFD. Later, these LDD females showed an improved metabolic flexibility (or the adaptation of fuel utilisation to fuel availability) 9 wk into the HFD regime (Chapter 5). At the end of the study one week later, LDD females exhibited a significantly lower absolute macrophage count in gWAT [LDD, 52.7 (22.5); HDD, 88.0 (18.3); mean and SD, $P<0.01$; Chapter 5]. However, because LDD females started off with lower macrophage infiltration, the estimated number of macrophages per 1000 adipocytes accumulated during the HFD period was not significantly different [LDD, 26.2 (22.6); HDD, 38.3 (18.3); mean and SD, Student's $t$ test $P=0.329]$. The female study in Chapter $\mathbf{3}$ was designed as an exact replication of Chapter $\mathbf{5}$ (with the exception of an InCa test in early life and an oral glucose tolerance test only conducted in Chapter 5), and there we found no programming of metabolic flexibility, but a significantly higher increase in adiposity (3.3\% or $1.7 \mathrm{~g} \mathrm{FM)} \mathrm{in} \mathrm{adult} \mathrm{LDD} \mathrm{compared} \mathrm{to} \mathrm{HDD} \mathrm{females} \mathrm{(Chapter} \mathbf{3}$ Table S1). Having seen that the programming evidence in Chapters $\mathbf{5}$ and $\mathbf{3}$ does not support each other, what can be concluded? At least for female BW and body composition data, the 


\section{CHAPTER 6}

data from both Chapters can be analysed together with the help of a linear mixed model, taking into account breeding batch as a random factor, while maximising the power to detect differences ( $n=36$ females). This statistical approach is appropriate for unbalanced designs and allows us to estimate the effects of post-weaning diet after adjusting for the random effects affecting different animal batches. The analysis indicates that there was no programming of BW, FM, or LM by LDD us HDD. With respect to metabolic flexibility, this direct comparison cannot be made directly because the challenge tests used to measure this parameter were different. In Chapter 5, we assessed metabolic flexibility as the RER increase in fasted mice after voluntary consumption of a solid food pellet containing starch (as the sole carbohydrate source), fat, and protein. In Chapter 3, a liquid mixed meal containing glucose and fat was administered by oral gavage instead. One could argue that the former type of challenge is more prone to chance findings because of its higher intersubject coefficient of variation (Chapter 5: 26\%; Chapter 3: 11\%; CV for RER), but both tests had similar intrasubject CV (Chapter 5: 7.1\%; Chapter 3: 7.4\%). We explicitly designed the nutritional challenge in Chapter $\mathbf{3}$ to contain only glucose and fat because the direct effects of lowly us highly digestible starches are known to target carbohydrate and fat metabolism ${ }^{64,65,79}$, and so indicate parameters that may be amenable to programming. However, the inclusion of protein in nutritional stress challenges in human studies has been proposed because of its potential to modulate metabolic processes (e.g. suppression of lipolysis) related to metabolic health ${ }^{80}$. It is therefore possible that one of the key processes behind programming of metabolic flexibility by LDD us HDD in Chapter $\mathbf{5}$ was related to protein metabolism, and that this difference was not apparent in Chapter $\mathbf{3}$ because protein was omitted in the challenge. The form of glucose included in the challenge might also be consequential. As discussed above for the direct effects of starch digestibility, dietary glucose oxidation was not influenced after 3 wk of intervention with lowly us highly digestible starch in male mice ${ }^{65}$, while we found a difference in dietary starch oxidation in Chapter 4. Our results remain inconclusive for metabolic flexibility in general, but the data suggests that LDD-programmed females are particularly efficient at adapting their fuel use strategy to glucose availability when provided as starch.

The consequences of early post-weaning LDD us HDD on adult metabolic phenotypes have not been investigated previously. Gugusheff et al. investigated the effects of maternal intake of a lowly us a highly digestible starch diet during pregnancy and lactation in rats. The findings were generally restricted to metabolic outcomes at weaning, showing lower visceral adiposity, lower hepatic lipid content, and improved glucose tolerance ${ }^{81}$. Only young adult female, but not male, offspring of dams fed a lowly digestible starch diet had a reduced hepatic expression of PI3K-p85 mRNA, speculated by the authors to eventually curb hepatic fat storage ${ }^{81}$. However, the experiment of Gugusheff et al. was not designed to test the direct and long-term effects of post-weaning starches separately, as offspring also had access to the mother's diet during weaning and the starch intervention continued until the end of the experiment, very early into adulthood. In support that the form of carbohydrates could program health outcomes much later in life, are the differences in longevity and reproductive success of female, but not male, offspring found when dams were fed a combination of glucose and fructose us sucrose ${ }^{82}$. When understood as proofof-concept studies that the form of carbohydrate (linear us branched glucose polymers, 
monosaccharides us disaccharides) can programme later life health, and that this can be sex-dependent, these two studies are compatible with our conclusions.

Although there is little evidence to directly compare our results, the outcomes we found to be susceptible to metabolic programming by the post-weaning diet (FM and metabolic flexibility), have been shown to be programmed by perinatal nutrition in rodent model $s^{83,84}$. The notion that early life influences can alter disease risk, or the developmental origins of health and disease $(\mathrm{DOHaD})$ paradigm ${ }^{85}$, is increasingly applied to the post-weaning period $^{86}$. There is substantial evidence for programing by nutritional and non-nutritional insults during the immediate post-weaning and adolescent stages on behavioural, cognitive, and neuro-endocrinological outcomes ${ }^{87-89}$, and the evidence for obesity and related metabolic outcomes is also accumulating ${ }^{90-93}$. Within the DOHaD field, it is widely accepted that similar programming outcomes induced by different insults may reflect shared mechanisms ${ }^{94}$. These mechanisms can be conveniently divided into proximate (or physiological, those that operate in the recent past, e.g. epigenetic mechanisms) and ultimate (or the processes that explain how the $\mathrm{DOHaD}$ phenomenon was selected for during evolution) ${ }^{95}$. Next to epigenetic programming of gene expression and accelerated cellular aging, tissue structure changes have been demonstrated as proximate mechanisms for metabolic programming ${ }^{9699}$. Changes in tissue structure may be especially important in programming by starches. One of the strongest direct effects of LDD was to increase small intestinal length and weight in both sexes (Chapter 5), perhaps as a consequence of relative caloric restriction with the less energy dense $\operatorname{LDD}^{100}$, or the trophic effects of SCFAs derived from LDD ${ }^{101}$. Although the length and weight of the small intestine were not significantly different between LDD and HDD adult mice at the end of the HFD period, there seems to be some relationship between small intestinal weight and FM gain on HFD. LDD females that gained more FM during HFD feeding also had a higher small intestinal weight after HFD feeding (Pearson correlation, $r^{2}=0.69, P<0.001, n=12$ ); this correlation was less significant in HDD females $\left(r^{2}=0.43, P<0.05, n=12\right)$ and was absent in males. Small intestinal weight of adult HDD females was on average $23 \%$ higher compared to the group culled at the end on the post-weaning intervention, whereas adult LDD females had a 3\% lighter small intestine compared to the young LDD group. This suggests that LDD females had a full-grown intestine already before the HFD, compared to HDD females where this organ continued to grow. If the earlier maturation of the small intestine by LDD us HDD led to enhanced nutrient absorption, this could partly explain the higher FM of adult LDD females in Chapter 3. Accordingly, FI completely equalised during the 1st week of HFD feeding and remained similar between groups, showing that the changes in FM were at least not mediated by an altered ingestive behaviour.

Regarding the "mismatch" hypothesis of $\mathrm{DOHaD}$, that states that differences between the environmental factors to which an organism is adapted and the environment extant later can affect fitness, two possibilities come to mind. Firstly, a mismatch between the anatomical and physiological adaptations to the foetal or suckling environments (e.g. influenced by the fermentable carbohydrates or the Gl of chow consumed by the dam or the dam's milk) could have made such changes maladaptive when the offspring were switched to the HDD (with no fermentable carbohydrates and possibly of high $\mathrm{Gl}$ ). This type of 
developmental mismatch has also been proposed by Gugusheff et al. ${ }^{81}$, and I would add that it is also a case of evolutionary mismatch. Second, if developmental plasticity in mice is still operative up till mid-adolescence, the mismatch may have happened between the phenotype adapted to LDD (less energy dense, possibly of low $\mathrm{Gl}$ ) during post-weaning and the later HFD (rich in energy and with highly digestible carbohydrates). This could explain why HDD females resisted body fat deposition on a HFD relative to LDD females in Chapter $\mathbf{3}$. Although this kind of developmental mismatch is mostly discussed in the context of foetal undernutrition and postnatal overnutrition in the DOHaD literature ${ }^{86,102,103}$, the mismatch hypothesis may operate at later nutritional transitions as long as there is some degree of developmental plasticity.

We are only beginning to understand the potential of starches to shape the developmental trajectory of an organism. In this thesis, different phenotypic outcomes in adult mice who consumed LDD us HDD from weaning to mid-adolescence, and then switched to a HFD until 15 wk of age, were considered as evidence of metabolic programming. We focussed on WAT based on previous observations in our laboratory showing strong direct effects of LDD us HDD on this organ ${ }^{79,104}$. But is that all there is to programming by starches? By no means. Firstly, there may be deeper or more nuanced effects in the organs that we found to be targeted by starches. Adipogenesis in rodents can continue at least until puberty ${ }^{105,106}$, and acetate, a SCFA increased directly by LDD, might induce adipogenesis ${ }^{107}$. This calls for measurements of cellularity in WAT, which requires complex techniques. The implications of macrophage infiltration in gWAT also await further exploration. Although we tested a selection of molecular markers in gWAT (Chapter 5), this type of analysis did not suggest that macrophages in this tissue in adult HDD females were pro-inflammatory (a more powerful approach could be, for instance, flow cytometry of macrophages isolated from fresh tissue). Nevertheless, at least one study suggests that eWAT macrophages in mice directly fed a lowly digestible starch may be pro-inflammatory ${ }^{108}$. Secondly, other organs and tissues (i.e. cardiovascular, kidney, liver, brain, pancreas, skeletal muscle ${ }^{109}$ ) are known to be susceptible to metabolic programming. For instance, the pancreas undergoes remodelling until four weeks after birth in rats ${ }^{110}, \beta$-cell mass is maintained by SCFA (directly increased by LDD) through inhibition of apoptosis ${ }^{107}$, and brain and colon can undergo epigenetic modifications up until early adulthood ${ }^{111,112}$.

Our findings that the metabolic flexibility and adiposity of the adult are influenced by the digestibility of starches consumed earlier in life thus raise new questions: Could other organs and tissues be programmed by starches? Could stronger effects of post-weaning diet be observed when the animals are followed into middle age? Could effects on other health outcomes be observed in males? The way forward in answering these questions becomes easier when one has identified clear direct effects in both sexes, and then follows those leads in longer studies. A more detailed approach is offered in the closing section of this Discussion.

Implications of starch digestibility for human health

Metabolic flexibility, as tested by refeeding challenges, is considered a signature of metabolic health. This measurement integrates the digestion, absorption, distribution, peripheral 
uptake, and ultimately the oxidation of the nutrients contained in a meal. In the transition from net fat oxidation during fasting to net glucose oxidation after a high carbohydrate meal, large flexibility may indicate optimal insulin sensitivity ${ }^{113}$, while decreased flexibility may precede the development of glucose intolerance ${ }^{114}$. Further, being metabolically flexible is a component of phenotypic flexibility, or the capacity of an organism to recover homeostasis in the face of metabolic challenges ${ }^{32}$, and is closely linked to WAT health ${ }^{115}$. On the other hand, body fat mass might be one of the factors contributing to cardiometabolic risk $^{116}$. The programmed increase in FM by LDD was not significant when taking all the data together however, and some have argued that lower macrophage infiltration in WAT is a feature of metabolically healthy obesity ${ }^{117}$. Taken together, our data would seem to support the view that LDD-programmed females were metabolically fitter.

To what extent could we extrapolate our findings in this mouse model to humans? To appreciate the translational value of our findings we should consider two things: first, how well the developing mouse resembles human ontogeny, and second, how is the exposure to lowly or highly digestible starches in young humans. The ontogenetic window of WAT, the organ we mostly focussed on in this thesis, is different in mice and humans. Mice have an underdeveloped adipose tissue at birth (2.1\% fat), while humans are born with a more developed adipose tissue (15\% fat $)^{118}$. However, mouse WAT development resembles that of humans in that both retain the capacity to increase adipocyte number during childhood and adolescence ${ }^{119,120}$. To the extent that the ontogenetic windows of mice and humans allow us to make a proper comparison, the beginning of our dietary starch intervention in post-weaning mice could represent an earlier developmental period in humans, possibly covering the very early postnatal period known as the first 1000 days of life, and extend until puberty, at least with regard to WAT development.

Since it is possible that the digestibility of the first starches consumed in life influences health in the long run also in humans, how does that fit into the current recommendations for carbohydrate intake for the developing human? Surprisingly, not at all. Currently, there are no science-based recommendations for starch intake for infants and toddlers, and it is assumed that carbohydrate intake after 2 years of age should follow to the recommendations for adults ${ }^{121}$. The current advice of WHO/FAO is to limit added sugars to $<10 \%$ of total energy intake (based on risk of developing caries and taste preferences) $)^{121,122}$ and, if anything, we know that these recommendations are not always met ${ }^{122,123}$. The closest thing we have to proper recommendations for starch intake is the recently issued Early Nutrition Project consensus, stating that there is no evidence for non-digestible carbohydrates added to infant formula to be protective against later obesity risk ${ }^{124}$. The lack of recommendations may in part be because data on starch intake by infants and toddlers is extremely limited. We only know that European infants younger than 1 year of age consume about $15 \%$ of their energy intake as starch, and children older than two years $23 \%$, but what type of starches these are is much less clear ${ }^{122}$. For babies, these are likely highly digestible, because only precooked and gelatinised starch can be included in infant formulas according to current EU Directives, but the types of starches included in complementary foods are not restricted ${ }^{122}$. This is compounded by the fact that the digestibility of starches largely depends on their origin and processing methods, such 
as cooking and cooling, making it very difficult to precisely estimate the exposure of the toddlers and children to lowly and highly digestible starches. As an alternative way to compare our model to human feeding practices early in life, the post-weaning HDD could mimic the premature weaning of a baby onto a high carbohydrate diet because of its purported high GI. This comparison is not sufficient however, as it does not take the effects of fermentation into account, and because $\mathrm{Gl}$ is a poor indicator of carbohydrate quality compared to dietary fibre ${ }^{125}$. Clearly, there is ample room to expand the recommendations for carbohydrate intake for babies and children. These recommendations need be informed by empirical evidence and will require a better recognition of digestibility as a measure of carbohydrate quality.

\section{Conclusions}

Females and males are affected differently by starches both directly and in the long-term. Direct exposure to LDD us HDD leads to lower inflammation and smaller adipocyte sizes in the gonadal adipose depot in females, and to decreased fat mass in males. In the long run, LDD consumed from weaning until mid-adolescence may program the female organism for a better metabolic flexibility or for higher adiposity during adulthood compared to HDD. The programming effects we observed were subtle, and further mechanistic studies are needed to substantiate them. Finally, our results strengthen the evidence that childhood and adolescence are important periods of development during which metabolic programming can take place.

\section{An Experimental Approach for Future Studies on Metabolic Programming}

While the importance of childhood and adolescence as critical periods of development are increasingly recognised in the $\mathrm{DOHaD}$ field ${ }^{126-128}$, most of the research efforts in experimental models have been focussed on the perinatal period. This choice may be conscious and very logical because, let it be said, intervening early on likely works much better to prevent disease risk ${ }^{129}$. However, I argue that another reason why metabolic programming studies do not commonly target phases of development later than weaning is because $\mathrm{DOHaD}$ hypotheses are difficult to study after this stage. I can offer two possible sources where this difficulty comes from. First, environmental cues become integrated as development progresses, and the resulting phenotype is influenced by each one of these cues. This was demonstrated by Gluckman et al., when they showed that the hepatic gene expression response to leptin administration in the neonatal rat is directionally dependent on the in utero nutritional status (ad libitum us undernourished dams) ${ }^{130}$. Because environmental exposures during highly plastic windows of development can determine the organismal response to future cues, this implies that even minor alterations in the experimental conditions before weaning can lead to different conclusions about metabolic programming by the post-weaning diet. We have seen indications of this phenomenon in other experiments conducted in our laboratory ${ }^{90}$ and also the ones reported in this thesis (Chapters $\mathbf{3}$ and 5). Despite the study in Chapter $\mathbf{3}$ was designed as an exact replication of Chapter $\mathbf{5}$ (with the exception of an InCa and an oral glucose tolerance test only conducted 
in Chapter 5), FM was programmed only in Chapter 3 (LDD > HDD). Moreover, the same HFD generally induced obesity more in Chapter 3 females [Chapter 3, 32 (22, 42); Chapter 5, $16(9,31) \%$ fat, median and range; Mann Whitney test, $P<0.0001]$, indicating that mice in Chapter $\mathbf{3}$ were more susceptible to the obesogenic environment. We know that at least two experimental variables may have affected different batches of mice at different stages of development: the breeding chow and the photoperiod. Since the chow provider stopped their services in the period between the studies reported in Chapters $\mathbf{5}$ and 3, we were forced to switch to another provider and thus chow composition. Although the chow diet of dams in all of our studies contained about $25 \%$ energy as protein, we later realized that the protein in the breeding chow in Chapter $\mathbf{3}$ was mainly of meat and dairy origin, while that in Chapter $\mathbf{5}$ came exclusively from grains. Also, the energy density of the breeding chow in Chapter $\mathbf{3}$ was considerably lower than that in Chapter $\mathbf{5}$ (13 us $17.3 \mathrm{~kJ} \mathrm{~g}^{-1}$ ). This is relevant because protein (of which almost only casein has been studied ${ }^{94}$ ) and energy restriction at various levels during gestation or lactation are known to affect metabolic outcomes like body weight and food intake in the offspring ${ }^{131}$. The chow in Chapter 5 also had a slightly lower methionine content ( $4 \mathrm{~g} \mathrm{~kg}^{-1}$ us $\left.5 \mathrm{~g} \mathrm{~kg}^{-1}\right)$, and methionine levels in the maternal diet might impact hepatic gene methylation in the foetus, which could in turn affect gene expression during organogenesis and eventually the phenotype of the offspring ${ }^{94}$. In addition, the chow used in Chapter $\mathbf{3}$ contained less fibre (2.7\%, from wheat and corn) compared to the chow in Chapter 5 (3.5\%, from wheat, oats, and alfalfa), which also suggests a very different fibre profile in terms of soluble and insoluble fibre, with potential consequences for the gut microbiome. Finally, some bioactive ingredients like phytoestrogens from alfalfa or linseed ${ }^{132,133}$ in Chapter 5's chow could have had metabolic effects in the dams and their offspring. The other environmental factor that could have influenced our results was an unfortunate malfunction of the light schedule during the study in Chapter 3, when a 3 h extension of the light regime happened during the first week of suckling and around conception, potentially misaligning the circadian system of dams and pups. Whether the 3 extra hours of light exposure were sufficient to disrupt the circadian system is difficult to estimate and rather debatable, but any deviations in the light regime are always strictly advised against in animal husbandry since light is a powerful entrainer of biological rhythms. The effects of chronodisruption during the perinatal period can compromise maternal care ${ }^{134}$, and circadian disruption during pregnancy is also known to affect foetal adrenal development which in turn may affect disease risk later in life ${ }^{135}$. Indeed, the statistical conclusions depended on the animal breeding batch between and within Chapters, and this factor accounted for $62 \%$ of the variance of the random effects in the whole data set. The bottom line is, even when we included littermate controls and further stratified mice by BW at weaning, if these environmental exposures had the power to change the response to later cues (LDD, HDD, and HFD) then these measures were likely ineffective.

The other reason why DOHaD hypotheses may be difficult to test in childhood and adolescence may be because these developmental windows are not very well defined in animal models. Mice do not have a childhood per $\mathrm{se}^{29}$. Apart from the generally straightforward considerations of sexual maturity and although adolescence is better defined in rodents than in other mammal models ${ }^{136}$, adolescence in rodents is defined by 
neurobehavioural characteristics ${ }^{136}$, while adolescence in humans is characterised by both psychological and metabolic aspects ${ }^{128}$. Moreover, the precise periods of developmental plasticity have not been mapped for all organ systems. This implies that further mapping of developmental plasticity and comparable assessment, in mouse and humans, should be part of programming studies in mice. This will inform our interpretations of effects in model organisms and strengthen the value of $\mathrm{DOHaD}$ as a theory.

I would like to propose a two-step experimental approach to make further progress in metabolic programming studies based on post-weaning nutrition, including the exploration of mechanisms. One strategy is to continue to conduct reductive, proof-of-concept studies in both sexes, like in this thesis. A useful first step is to characterise the direct effects of nutritional insults, although some may escape phenotyping if they are subtle and only become obvious in extreme environments. These direct effects may result from predictive or immediate adaptive responses of the organism to the environment, and may give a hint about what systems may become programmed. It will be important to consistently target the intended developmental window of intervention in these studies which could imply, for example, the separation of the dam's diet from the pups during lactation by means of special cages (where pups and dams can be fed separately at least until 18 days postnatal), making sure that pups are weaned only onto the intervention diet and not onto chow ${ }^{137}$. These experiments should be followed by longer mechanistic studies that include attempts to revert the purported programmed outcomes. Additionally, this would clarify whether "permanence" of effects defines metabolic programming as proposed by some ${ }^{99,138}$, but not fully supported by evidence ${ }^{139}$, and improve our understanding of the programming mechanisms that are at play as developmental plasticity declines. To effectively and efficiently establish programming effects by post-weaning nutrition, all of these experiments should be highly controlled and standardised and ensure adequate statistical power, not necessarily by using more animals, but by using precise and integrative phenotyping tools.

At a later stage, one could release the control of some variables, attempt to resemble human diets more closely, and test the established programming effects in more ecologically relevant conditions. Such experiments could be conducted in semi-natural enclosures ${ }^{82}$, making the timing of weaning more natural ${ }^{140,141}$ and mimicking the babyled weaning practice recommended in humans ${ }^{142}$. These less controlled but more realistic conditions would likely highlight the most consequential nutritional cues that can program metabolism after weaning. More importantly, it would also allow us to evaluate how nutritional cues play out under the evolutionary dogmas of $\mathrm{DOHaD}$, e.g. developmental adaptations hypothesised to preserve survival up until reproductive age could be tested directly. Ultimately, this take on developmental programming would help achieve the holistic approach to nutritional science advocated by some scholars ${ }^{143}$.

\section{Conclusions}

In 2015, the year when this PhD project started, the Sustainable Development Goals (SDGs) were launched. Together with our work, future studies trying to navigate the complexity of nutritional programming phenomena will hopefully contribute to reach SDG \#3: "Good health and well-being for people". Nutrition during childhood and adolescence 
may strongly impact later life health, but experimental evidence for the programming effects of nutrition during these stages is in short supply. Likely, this is because DOHaD hypotheses are difficult to test once the responses to all previous environmental cues have already been integrated. We should however embrace this complexity and keep on researching, because what this complexity is really telling us is that there are many ways to intervene.

\section{General Conclusions}

We have shown that a mouse indirect calorimetry system extended with additional gas analysers can be used to study gut microbial activity $\left(\mathrm{H}_{2}\right.$ and $\left.\mathrm{CH}_{4}\right)$ and the oxidation of exogenous vs endogenous substrates $\left({ }^{13} \mathrm{CO}_{2}\right.$ and $\left.{ }^{12} \mathrm{CO}_{2}\right)$. Our studies also showed that, although the effects of a lowly digestible starch diet are generally beneficial, female and male mice are differently affected by dietary starch digestibility. Finally, dietary starch digestibility during the early post-weaning period seem to program metabolic health later in life especially in females, although the observed effects were subtle and need to be confirmed and substantiated mechanistically. 


\section{References}

Smith, N. W., Shorten, P. R., Altermann, E. H., Roy, N. C. \& McNabb, W. C. Hydrogen cross-feeders of the human gastrointestinal tract. Gut microbes, 1-19 (2018).

Levitt, M. D. Production and excretion of hydrogen gas in man. N. Engl. J. Med. 281, 122-127 (1969).

Carbonero, F., Benefiel, A. C. \& Gaskins, H. R. Contributions of the microbial hydrogen economy to colonic homeostasis. Nat. Reu. Gastroenterol. Hepatol. 9, 504-518 (2012).

Andrieux, C., Pacheco, E. D., Bouchet, B., Gallant, D. \& Szylit, O. Contribution of the digestive tract microflora to amylomaize starch degradation in the rat. Brit. J. Nutr. 67, 489-99 (1992).

Fetissov, S. O. Role of the gut microbiota in host appetite control: bacterial growth to animal feeding behaviour. Nat. Reu. Endocrinol. 13, 11-25 (2017).

Key, F. B. \& Mathers, J. C. Digestive adaptations of rats given white bread and cooked haricot beans (Phaseolus vulgaris): large-bowel fermentation and digestion of complex carbohydrates. Br. J. Nutr. 74, 393-406 (1995).

Walker, A. W. et al. Dominant and diet-responsive groups of bacteria within the human colonic microbiota. ISME J. 5, 220-230 (2011).

Levrat, M. A., Remesy, C. \& Demigne, C. Very acidic fermentations in the rat cecum during adaptation to a diet rich in amylase-resistant starch (crude potato starch). J. Nutr. Biochem. 2, 31-36 (1991).

David, L. A. et al. Host lifestyle affects human microbiota on daily timescales. Genome biology 15 , R89 (2014).

Padmanabhan, P., Grosse, J., Asad, A. B., Radda, G. K. \& Golay, X. Gastrointestinal transit measurements in mice with ${ }^{99 m}$ Tc-DTPA-labeled activated charcoal using NanoSPECT-CT. EJNMMI Res. 3, 60 (2013).

Tuboly, E. et al. Excessive alcohol consumption induces methane production in humans and rats. Sci. Rep. 7, 7329 (2017).

Tuboly, E. et al. Determination of endogenous methane formation by photoacoustic spectroscopy. J. Breath Res. 7, 046004 (2013).

Tuboly, E. et al. Methane biogenesis during sodium azide-induced chemical hypoxia in rats. Am. J. Physiol., Cell Physiol. 304, C207-214 (2013).

\section{Swanson, K. S. et al. Phylogenetic and gene-centric metagenomics of the canine intestinal} microbiome reveals similarities with humans and mice. ISME J. 5, 639-649 (2011).

Laverdure, R., Mezouari, A., Carson, M. A., Basiliko, N. \& Gagnon, J. A role for methanogens and methane in the regulation of GLP-1. Endocrinol. Diabetes Metab. 1, e00006 (2018).

Hamer, H. M., De Preter, V., Windey, K. \& Verbeke, K. Functional analysis of colonic bacterial metabolism: relevant to health? Am. J. Physiol. Gastrointest. Liver Physiol. 302, G1-9 (2012).

Nakamura, N., Lin, H. C., McSweeney, C. S., Mackie, R. I. \& Gaskins, H. R. Mechanisms of microbial hydrogen disposal in the human colon and implications for health and disease. Annu. Reu. Food Sci. Technol. 1, 363-395 (2010).

Zheng, X. F., Sun, X. J. \& Xia, Z. F. Hydrogen resuscitation, a new cytoprotective approach. Clin. Exp. Pharmacol. Physiol. 38, 155-163 (2011).

Nakao, A., Toyoda, Y., Sharma, P., Evans, M. \& Guthrie, N. Effectiveness of hydrogen rich water on antioxidant status of subjects with potential metabolic syndrome-an open label pilot study. J. Clin. Biochem. Nutr. 46, 140-149 (2010).

Kajiyama, S. et al. Supplementation of hydrogen-rich water improves lipid and glucose metabolism in patients with type 2 diabetes or impaired glucose tolerance. Nutr. Res. 28, 137-143 (2008).

Makki, K., Deehan, E. C., Walter, J. \& Backhed, F. The impact of dietary fiber on gut microbiota in host health and disease. Cell Host Microbe 23, 705-715 (2018). 
Attene-Ramos, M. S., Wagner, E. D., Plewa, M. J. \& Gaskins, H. R. Evidence that hydrogen sulfide is a genotoxic agent. Mol. Cancer Res. 4, 9-14 (2006).

Wang, R. Two's company, three's a crowd: can $\mathrm{H}_{2} \mathrm{~S}$ be the third endogenous gaseous transmitter? FASEB J. 16, 1792-1798 (2002).

Goubern, M., Andriamihaja, M., Nubel, T., Blachier, F. \& Bouillaud, F. Sulfide, the first inorganic substrate for human cells. FASEB J. 21, 1699-1706 (2007).

Soriano, R. N. et al. Endogenous peripheral hydrogen sulfide is propyretic: its permissive role in brown adipose tissue thermogenesis in rats. Exp. Physiol. 103, 397-407 (2018).

Gerrits, W. J. J., Borne, J. J. G. C. v. d. \& Labussière, E. in Indirect Calorimetry Ch. 1, 19-34 (Wageningen Academic Publishers, Wageningen, 2015).

Poppitt, S. D. et al. Circadian patterns of total 24-h hydrogen and methane excretion in humans ingesting nonstarch polysaccharide (NSP) diets and the implications for indirect calorimetric and $D_{2}$ ${ }^{18} \mathrm{O}$ methodologies. Eur. J. Clin. Nutr. 50, 524-534 (1996).

Tulley, R. T. et al. Comparative methodologies for measuring metabolizable energy of various types of resistant high amylose corn starch. J. Agric. Food Chem. 57, 8474-8479 (2009).

Brust, V., Schindler, P. M. \& Lewejohann, L. Lifetime development of behavioural phenotype in the house mouse (Mus musculus). Front. Zool. 12 Suppl 1, S17 (2015).

Keenan, M. J. et al. Role of resistant starch in improving gut health, adiposity, and insulin resistance. Adv. Nutr. 6, 198-205 (2015).

Mushref, M. A. \& Srinivasan, S. Effect of high fat-diet and obesity on gastrointestinal motility. Ann. Transl. Med. 1, 14 (2013).

Picó, Serra, Rodríguez, Keijer \& Palou. Biomarkers of nutrition and health: new tools for new approaches. Nutrients 11, 1092 (2019).

Mosora, F. et al. Quantitative evaluation of the oxidation of an exogenous glucose load using naturally labeled ${ }^{13}$ C-glucose. Metabolism 25, 1575-1582 (1976).

Welch, K. C., Jr., Peronnet, F., Hatch, K. A., Voigt, C. C. \& McCue, M. D. Carbon stable-isotope tracking in breath for comparative studies of fuel use. Ann. N. Y. Acad. Sci. 1365, 15-32 (2016).

Ishihara, K. et al. A soybean peptide isolate diet promotes postprandial carbohydrate oxidation and energy expenditure in type II diabetic mice. J. Nutr. 133, 752-757 (2003).

McCue, M. D. \& Pollock, E. D. Measurements of substrate oxidation using ${ }^{13} \mathrm{CO}_{2}$-breath testing reveals shifts in fuel mix during starvation. J. Comp. Physiol. B. 183, 1039-1052 (2013).

Ishihara, K. et al. Use of ${ }^{13} \mathrm{C}$-labeled glucose for measuring exogenous glucose oxidation in mice. Biosci. Biotechnol. Biochem. 66, 426-429 (2014).

Ishihara, K. et al. Electrolyte-free milk protein solution influences sodium and fluid retention in rats. J. Nutr. Sci. 2, e8 (2013).

Zhou, J. et al. Dietary resistant starch upregulates total GLP-1 and PYY in a sustained day-long manner through fermentation in rodents. Am. J. Physiol. Endocrinol. Metab. 295, E1160-1166 (2008).

Friedrich, M., Petzke, K. J., Raederstorff, D., Wolfram, S. \& Klaus, S. Acute effects of epigallocatechin gallate from green tea on oxidation and tissue incorporation of dietary lipids in mice fed a high-fat diet. Int. J. Obes. 36, 735-743 (2012). obesity in mice by decreasing energy absorption and increasing fat oxidation. Int. J. Obes. 29, 615623 (2005). 
43 Bergouignan, A., Schoeller, D. A., Votruba, S., Simon, C. \& Blanc, S. The acetate recovery factor to correct tracer-derived dietary fat oxidation in humans. Am. J. Physiol. Endocrinol. Metab. 294, E645653 (2008).

$44 \quad$ Antoun, E. et al. The $\left[1-{ }^{13} \mathrm{C}\right]$ acetate recovery factor to correct tracer-derived dietary fat oxidation is lower in overweight insulin-resistant subjects. E Spen Eur. E J. Clin. Nutr. Metab. 5, e173-e179 (2010).

Bessesen, D. H., Rupp, C. L. \& Eckel, R. H. Dietary-fat is shunted away from oxidation, toward storage in obese Zucker rats. Obes. Res. 3, 179-189 (1995).

Bessesen, D. H., Bull, S. \& Cornier, M. A. Trafficking of dietary fat and resistance to obesity. Physiol. Behav. 94, 681-688 (2008).

Jackman, M. R., Kramer, R. E., MacLean, P. S. \& Bessesen, D. H. Trafficking of dietary fat in obesityprone and obesity-resistant rats. Am. J. Physiol. Endocrinol. Metab. 291, E1083-1091 (2006).

Rynders, C. A., Pereira, R. I., Bergouignan, A., Kealey, E. H. \& Bessesen, D. H. Associations among dietary fat oxidation responses to overfeeding and weight gain in obesity-prone and resistant adults. Obesity 26, 1758-1766 (2018).

Speakman, J. R. \& Thomson, S. C. Validation of the labeled bicarbonate technique for measurement of short-term energy expenditure in the mouse. Z. Ernahrungswiss 36, 273-277 (1997).

Duivenvoorde, L. P., van Schothorst, E. M., Swarts, H. J. \& Keijer, J. Assessment of metabolic flexibility of old and adult mice using three noninvasive, indirect calorimetry-based treatments. J. Gerontol. A Biol. Sci. Med. Sci. 70, 282-293 (2015).

Elia, M. \& Livesey, G. Energy expenditure and fuel selection in biological systems: the theory and practice of calculations based on indirect calorimetry and tracer methods. World Reu. Nutr. Diet 70, 68-131 (1992).

Schutz, Y. \& Ravussin, E. Respiratory quotients lower than 0.70 in ketogenic diets. Am. J. Clin. Nutr. 33, 1317-1319 (1980).

Ferrannini, E. The theoretical bases of indirect calorimetry: q review. Metabolism 37, 287-301 (1988).

54 Sonko, B. J., Prentice, A. M., Coward, W. A., Murgatroyd, P. R. \& Goldberg, G. R. Dose-response relationship between fat ingestion and oxidation: quantitative estimation using whole-body calorimetry and ${ }^{13} \mathrm{C}$ isotope ratio mass spectrometry. Eur. J. Clin. Nutr. 55, 10-18 (2001).

Flachs, P. et al. Synergistic induction of lipid catabolism and anti-inflammatory lipids in white fat of dietary obese mice in response to calorie restriction and n-3 fatty acids. Diabetologia 54, 2626-2638 (2011).

Bowe, J. E. et al. Metabolic phenotyping guidelines: assessing glucose homeostasis in rodent models. J. Endocrinol. 222, G13-25 (2014).

Shinozaki, K. et al. Dissociated oxygen consumption and carbon dioxide production in the postcardiac arrest rat: a novel metabolic phenotype. J. Am. Heart Assoc. 7, e007721 (2018).

Pereira, J. et al. Breath analysis as a potential and non-invasive frontier in disease diagnosis: an overview. Metabolites 5, 3-55 (2015).

59 Buszewski, B., Kesy, M., Ligor, T. \& Amann, A. Human exhaled air analytics: biomarkers of diseases. Biomed. Chromatogr. 21, 553-566 (2007).

Karl, T. et al. Human breath isoprene and its relation to blood cholesterol levels: new measurements and modeling. J. Appl. Physiol. (1985) 91, 762-770 (2001). concentrations during acute myocardial infarction. Lancet 337, 933-935 (1991). 
Campbell, G. J., Senior, A. M. \& Bell-Anderson, K. S. Metabolic effects of high glycaemic index diets: a systematic review and meta-analysis of feeding studies in mice and rats. Nutrients 9, 646 (2017).

Isken, F. et al. Impairment of fat oxidation under high-vs. low-glycemic index diet occurs before the development of an obese phenotype. Am. J. Physiol. Endocrinol. Metab. 298, E287-295 (2010).

Kabir, M. et al. Dietary amylose-amylopectin starch content affects glucose and lipid metabolism in adipocytes of normal and diabetic rats. J. Nutr. 128, 35-43 (1998).

Karastergiou, K. \& Fried, S. K. Cellular mechanisms driving sex differences in adipose tissue biology and body shape in humans and mouse Models. Adv. Exp. Med. Biol. 1043, 29-51 (2017).

Chen, X., McClusky, R., Itoh, Y., Reue, K. \& Arnold, A. P. X and Y chromosome complement influence adiposity and metabolism in mice. Endocrinology 154, 1092-1104 (2013).

Ingvorsen, C., Karp, N. A. \& Lelliott, C. J. The role of sex and body weight on the metabolic effects of high-fat diet in C57BL/6N mice. Nutr. Diabetes 7, e261 (2017).

Riddell, M. C. The endocrine response and substrate utilization during exercise in children and adolescents. J. Appl. Physiol. (1985) 105, 725-733 (2008).

Tarnopolsky, M. A. Gender differences in substrate metabolism during endurance exercise. Can. J. Appl. Physiol. 25, 312-327 (2000).

Falconer, D. S. Weight and age at puberty in female and male mice of strains selected for large and small body size. Genet. Res. 44, 47-72 (1984).

Link, J. C. \& Reue, K. Genetic basis for sex differences in obesity and lipid metabolism. Annu. Reu. Nutr. 37, 225-245 (2017).

Zeevi, D. et al. Structural variation in the gut microbiome associates with host health. Nature $\mathbf{5 6 8}$, 43-48 (2019).

Tang, W. H. \& Hazen, S. L. Microbiome, trimethylamine N-oxide, and cardiometabolic disease. Transl. Res. 179, 108-115 (2017).

Bennett, B. J. et al. Trimethylamine-N-oxide, a metabolite associated with atherosclerosis, exhibits complex genetic and dietary regulation. Cell Metab. 17, 49-60 (2013).

Della Torre, S. et al. Short-term fasting reveals amino acid metabolism as a major sex-discriminating factor in the liver. Cell Metab. 28, 256-267 e255 (2018).

Aiken, C. E. \& Ozanne, S. E. Sex differences in developmental programming models. Reproduction 145, R1-13 (2013).

van Schothorst, E. M., Bunschoten, A., Schrauwen, P., Mensink, R. P. \& Keijer, J. Effects of a highfat, low- versus high-glycemic index diet: retardation of insulin resistance involves adipose tissue modulation. FASEB ]. 23, 1092-1101 (2009).

Stroeve, J. H., van Wietmarschen, H., Kremer, B. H., van Ommen, B. \& Wopereis, S. Phenotypic flexibility as a measure of health: the optimal nutritional stress response test. Genes Nutr. 10, 459 (2015).

Gugusheff, J. et al. The effect of maternal and post-weaning low and high glycaemic index diets on glucose tolerance, fat deposition and hepatic function in rat offspring. J. Dev. Orig. Health Dis., 1-10 (2015).

Ruff, J. S. et al. Compared to sucrose, previous consumption of fructose and glucose monosaccharides reduces survival and fitness of female mice. J. Nutr. 145, 434-441(2015).

Huber, M. et al. How should we define health? BMJ 343, d4163 (2011). resistance to high fat diet-induced obesity. PLoS One 9, e104896 (2014). 
Koletzko, B. et al. The Power of Programming and the EarlyNutrition project: opportunities for health promotion by nutrition during the first thousand days of life and beyond. Ann. Nutr. Metab. 64, 187196 (2014).

Schmidt, M. V. Molecular mechanisms of early life stress--lessons from mouse models. Neurosci. Biobehav. Rev. 34, 845-852 (2010). inadequate diet during different stages of life in rat. Neurosci. Biobehav. Rev. 36, 1463-1480 (2012). behavior and hypothalamic pituitary adrenal axis at the onset of puberty in a sexually dimorphic manner. Neuroscience 153, 373-382 (2008).

Bouwman, L. M. S. et al. Replacing part of glucose with galactose in the postweaning diet protects female but not male mice from high-fat diet-induced adiposity in later life. J Nutr. nxzO28 (2019).

Oosting, A. et al. Effect of dietary lipid structure in early postnatal life on mouse adipose tissue development and function in adulthood. Br. J. Nutr. 111, 215-226 (2014).

He, Y. H. et al. Postweaning low-calcium diet promotes later-life obesity induced by a high-fat diet. J. Nutr. Biochem. 23, 1238-1244 (2012).

Minana-Solis Mdel, C. \& Escobar, C. Post-weaning protein malnutrition in the rat produces short and long term metabolic impairment, in contrast to earlier and later periods. Int. J. Biol. Sci. 4, 422-432 (2008).

94 Armitage, J. A., Khan, I. Y., Taylor, P. D., Nathanielsz, P. W. \& Poston, L. Developmental programming of the metabolic syndrome by maternal nutritional imbalance: how strong is the evidence from experimental models in mammals? J. Physiol. 561, 355-377 (2004).

Gluckman, P. D., Hanson, M. A. \& Buklijas, T. A conceptual framework for the developmental origins of health and disease. J. Deu. Orig. Health Dis. 1, 6-18 (2010).

Vickers, M. H. Early life nutrition, epigenetics and programming of later life disease. Nutrients $\mathbf{6}$, 2165-2178 (2014).

Reichetzeder, C., Dwi Putra, S. E., Li, J. \& Hocher, B. Developmental origins of disease - crisis precipitates change. Cell. Physiol. Biochem. 39, 919-938 (2016).

Sutton, E. F. et al. Developmental programming: state-of-the-science and future directions - summary from a Pennington Biomedical symposium. Obesity 24, 1018-1026 (2016).

99 Langley-Evans, S. C. Nutrition in early life and the programming of adult disease: a review. J. Hum. Nutr. Diet. 28 Suppl 1, 1-14 (2015).

Mitchell, S. E. et al. The effects of graded levels of calorie restriction: I. impact of short term calorie and protein restriction on body composition in the C57BL/6 mouse. Oncotarget 6, 15902-15930 (2015).

Bird, A. R., Brown, I. L. \& Topping, D. L. Starches, resistant starches, the gut microflora and human health. Curr. Issues Intest. Microbiol. 1, 25-37 (2000).

102 Hanson, M. A. \& Gluckman, P. D. Early developmental conditioning of later health and disease: physiology or pathophysiology? Physiol. Rev. 94, 1027-1076 (2014). disease: a life history and evolutionary perspective. Am. J. Hum. Biol. 19, 1-19 (2007).

van Schothorst, E. M., Bunschoten, A., Verlinde, E., Schrauwen, P. \& Keijer, J. Glycemic index differences of high-fat diets modulate primarily lipid metabolism in murine adipose tissue. Physiol. Genomics 43, 942-949 (2011). development of obesity in the rat. J. Nutr. 110, 2027-2034 (1980). 
Canfora, E. E., Meex, R. C. R., Venema, K. \& Blaak, E. E. Gut microbial metabolites in obesity, NAFLD and T2DM. Nat. Reu. Endocrinol. 15, 261-273 (2019).

108 Barouei, J. et al. Microbiota, metabolome, and immune alterations in obese mice fed a high-fat diet containing type 2 resistant starch. Mol. Nutr. Food Res. 61 (2017).

109 McMillen, I. C. \& Robinson, J. S. Developmental origins of the metabolic syndrome: prediction, plasticity, and programming. Physiol. Reu. 85, 571-633 (2005).

110 Fowden, A. L. \& Hill, D. J. Intra-uterine programming of the endocrine pancreas. Br. Med. Bull. 60 123-142 (2001).

111 Marco, A., Kisliouk, T., Weller, A. \& Meiri, N. High fat diet induces hypermethylation of the hypothalamic Pomc promoter and obesity in post-weaning rats. Psychoneuroendocrinology $\mathbf{3 8}$, 2844-2853 (2013).

112 Kellermayer, R. et al. Epigenetic maturation in colonic mucosa continues beyond infancy in mice. Hum. Mol. Genet. 19, 2168-2176 (2010).

Galgani, J. E., Moro, C. \& Ravussin, E. Metabolic flexibility and insulin resistance. Am. J. Physiol. Endocrinol. Metab. 295, E1009-1017 (2008).

Muoio, D. M. Metabolic inflexibility: when mitochondrial indecision leads to metabolic gridlock. Cell 159, 1253-1262 (2014).

Sparks, L. M. et al. Relation of adipose tissue to metabolic flexibility. Diabetes Res. Clin. Pract. 83, 3243 (2009).

Romero-Corral, A. et al. Normal weight obesity: a risk factor for cardiometabolic dysregulation and cardiovascular mortality. Eur. Heart. J. 31, 737-746 (2010).

Vegiopoulos, A., Rohm, M. \& Herzig, S. Adipose tissue: between the extremes. EMBO J. 36, 19992017 (2017).

Kuzawa, C. W. Adipose tissue in human infancy and childhood: an evolutionary perspective. Am. J. Phys. Anthropol. Suppl 27, 177-209 (1998).

Spalding, K. L. et al. Dynamics of fat cell turnover in humans. Nature 453, 783-787 (2008).

Salans, L. B., Cushman, S. W. \& Weismann, R. E. Studies of human adipose tissue. Adipose cell size and number in nonobese and obese patients. J. Clin. Invest. 52, 929-941 (1973).

Food and Agriculture Organization of the United Nations. Carbohydrates in Human Nutrition: Report of a Joint FAO/WHO Expert Consultation, Rome, 14-18 April 1997. (FAO, Rome, 1998).

Stephen, A. et al. The role and requirements of digestible dietary carbohydrates in infants and toddlers. Eur. J. Clin. Nutr. 66, 765-779 (2012).

Wang, Y., Guglielmo, D. \& Welsh, J. A. Consumption of sugars, saturated fat, and sodium among US children from infancy through preschool age, NHANES 2009-2014. Am. J. Clin. Nutr. 108, 868-877 (2018).

Koletzko, B. et al. Nutrition during pregnancy, lactation and early childhood and its implications for maternal and long-term child health: the Early Nutrition Project recommendations. Ann. Nutr. Metab. 74, 93-106 (2019).

Reynolds, A. et al. Carbohydrate quality and human health: a series of systematic reviews and metaanalyses. Lancet 393, 434-445 (2019).

Azzopardi, P. S. et al. Progress in adolescent health and wellbeing: tracking 12 headline indicators for 195 countries and territories, 1990-2016. Lancet 393, 1101-1118 (2019).f

Woods-Townsend, K. et al. LifeLab Southampton: a programme to engage adolescents with DOHaD concepts as a tool for increasing health literacy in teenagers -a pilot cluster-randomized control trial. J. Dev. Orig. Health Dis. 9, 475-480 (2018). 
Godfrey, K. M., Gluckman, P. D. \& Hanson, M. A. Developmental origins of metabolic disease: life course and intergenerational perspectives. Trends Endocrinol. Metab. 21, 199-205 (2010).

Gluckman, P. D. et al. Metabolic plasticity during mammalian development is directionally dependent on early nutritional status. P. Natl. Acad. Sci. USA 104, 12796-12800 (2007).

Pico, C., Palou, M., Priego, T., Sanchez, J. \& Palou, A. Metabolic programming of obesity by energy restriction during the perinatal period: different outcomes depending on gender and period, type and severity of restriction. Front. Physiol. 3, 436 (2012).

Hall, C., Tulbek, M. C. \& Xu, Y. Flaxseed. Adv. Food Nutr. Res. 51, 1-97 (2006).

Pellizzon, M. A. \& Ricci, M. R. The common use of improper control diets in diet-induced metabolic disease research confounds data interpretation: the fiber factor. Nutr. Metab. 15, 3 (2018). mother improves the offspring's pathological phenotype. J. Physiol. (Lond.) 596, 5757-5775 (2018).

Salazar, E. R. et al. Gestational chronodisruption leads to persistent changes in the rat fetal and adult adrenal clock and function. J. Physiol. (Lond.) 596, 5839-5857 (2018).

Spear, L. P. The adolescent brain and age-related behavioral manifestations. Neurosci. Biobehau. Reu. 24, 417-463 (2000).

Koopman, J. P., Scholten, P. M. \& Beynen, A. C. Hardness of diet pellets and growth of pre-weaned mice: separation of direct effects on the young and indirect effects mediated by the lactating females. Z. Versuchstierkd. 32, 257-260 (1989).

Li, M., Reynolds, C. M., Segovia, S. A., Gray, C. \& Vickers, M. H. Developmental programming of nonalcoholic fatty liver disease: the effect of early life nutrition on susceptibility and disease severity in later life. Biomed Res. Int. 2015, 437107 (2015).

139 Kasch, J. et al. Insulin sensitivity linked skeletal muscle $\mathrm{Nr} 4 a 1$ DNA methylation is programmed by the maternal diet and modulated by voluntary exercise in mice. J. Nutr. Biochem. (2018).

140 Hahn, P. Effect of premature weaning to different diets on the subsequent response to a dietary change. Biol. Neonate 32, 275-279 (1977).

141 Richter, S. H., Kastner, N., Loddenkemper, D. H., Kaiser, S. \& Sachser, N. A time to wean? Impact of weaning age on anxiety-like behaviour and stability of behavioural traits in full adulthood. PLoS One 11, e0167652 (2016).

142 Moorcroft, K. E., Marshall, J. L. \& McCormick, F. M. Association between timing of introducing solid foods and obesity in infancy and childhood: a systematic review. Matern. Child Nutr. 7, 3-26 (2011)

Penders, B. et al. Capable and credible? Challenging nutrition science. Eur. J. Nutr. 56, 2009-2012 (2017). 


\section{Summary}



Indirect calorimetry $(\ln C a)$ is an essential tool for human and animal studies of energy metabolism. InCa measures the metabolic gases consumed (oxygen, $\mathrm{O}_{2}$ ) and produced (carbon dioxide, $\mathrm{CO}_{2}$ ) by the organism to calculate energy expenditure (EE). The ratio of $\mathrm{CO}_{2}$ produced to $\mathrm{O}_{2}$ consumed, or the respiratory exchange ratio (RER), indicates substrate utilisation (carbohydrate, fat, and protein) at the whole body level. Significant improvements in commercial InCa systems for rodents have increased its accuracy and resolution, yet there have been few attempts to extend the technique by measuring other physiological gases. These gases can be of microbial origin, like hydrogen $\left(\mathrm{H}_{2}\right)$ and methane $\left(\mathrm{CH}_{4}\right)$, which result from fermentation by the gut microbiome. Furthermore, conventional InCa cannot distinguish between the oxidation of exogenous (e.g. dietary) and endogenous (stored in the body) substrates. Stable isotopic tracers, like ${ }^{13} \mathrm{C}$-enriched nutrients, make this distinction possible by analysis of the ratio of ${ }^{13} \mathrm{CO}_{2} /{ }^{12} \mathrm{CO}_{2}$ in expired air. These gases $\left(\mathrm{H}_{2}, \mathrm{CH}_{4},{ }^{13} \mathrm{CO}_{2},{ }^{12} \mathrm{CO}_{2}\right)$ are rarely measured in mouse studies and, when they are, they need expensive equipment and considerable time and effort.

Dietary starches can be lowly or highly digestible. Lowly digestible starches generally produce lower glycaemic responses, provide fermentative substrates for the intestinal microbiota, and are thought to prevent excessive adiposity and favour metabolic health. There are some indications that starch digestibility has different metabolic effects in females and males. The evidence for this is very limited and, where both sexes have been studied, little attention has been paid to other aspects of carbohydrate metabolism, besides oral glucose tolerance tests and static tests for glycaemia. Moreover, despite the potential of starches to impact metabolism, there are no evidence-based recommendations for starch intake for young children. This is important because early life nutrition has the power to condition the metabolic function of the organism in the long term, a phenomenon known as metabolic programming. It is not known whether starch digestibility can program the organism metabolically, neither whether programming by starches can occur in the post-weaning period, a period where the organism faces a dramatic change in dietary macronutrient composition and density.

This thesis aimed to 1) develop an show the added value of an extended mouse metabolic phenotyping tool based on $\mathrm{InCa}$ for the real-time study of microbiota activity and the oxidation of exogenous us endogenous substrates; and 2) to apply this tool to study the direct and metabolic programming effects of starches consumed during the early postweaning period.

In Chapter 2, we aimed to examine whether we would be able to study microbiota activity non-invasively, continuously and in real time by extending a commercial InCa system (elnCa) with $\mathrm{H}_{2}$ and $\mathrm{CH}_{4}$ sensors (elnCa). Hydrogen production was circadian and depended on food intake and starch digestibility, as tested in mice fed a lowly (LDD) or a highly digestible-starch diet (HDD). Hydrogen production explained $20 \%$ of the variation 
in faecal bacterial composition, and correlated with specific bacterial genera known to produce $\mathrm{H}_{2}$ in vitro. No $\mathrm{CH}_{4}$ production could be measured, and this was consistent with the absence of faecal methanogenic archaea. We concluded that elnCa is a useful tool to study diet-microbiota-host interactions in real time.

We then incorporated ${ }^{13} \mathrm{CO}_{2}$ and ${ }^{12} \mathrm{CO}_{2}$ sensors into the same elnCa system and aimed to demonstrate their added value in Chapter 3. The system detected to differences in ${ }^{13} \mathrm{CO}_{2}$ enrichment based on the natural ${ }^{13} \mathrm{C}$ enrichment of the diet and daily food intake patterns. By combining ${ }^{13} \mathrm{CO}_{2}$ enrichment and conventional InCa data, we were able to quantify the oxidation rates of ${ }^{13} \mathrm{C}$ glucose or ${ }^{13} \mathrm{C}$ palmitate ingested by lean and obese mice, separately from total (exogenous + endogenous) glucose and fat oxidation rates. The oxidation of ingested palmitate was negatively correlated to the animal's fat mass and positively correlated to metabolic flexibility. We concluded that enabling ${ }^{13} \mathrm{CO}_{2}$ enrichment analysis in elnCa makes it a powerful tool for the quantification of specific substrate oxidation in physiological and pathophysiological conditions .

In Chapter 4, we aimed to test whether the capacity to oxidise the starch molecule is influenced by prior exposure to an LDD or an HDD, and if this effect is similar for both females and males. The oxidation of a highly digestible starch bolus was higher in mice that consumed LDD for 3 weeks prior. This effect was larger in females than in males, as shown by the faster starch oxidation kinetics of LDD us HDD females during the early postprandial period, not present in males. However, LDD males exhibited a constantly higher RER, both in fasting conditions and during the postprandial period after consumption of the starch bolus. Small intestinal amylase levels did not explain the higher starch oxidation of LDD us HDD females. From these data we concluded that short-term consumption of lowly digestible starch enhances the oxidation of the starch molecule, especially in females.

In Chapter 5, we aimed to retest some of the direct effects of starch digestibility and to test whether LDD us HDD consumed in early post-weaning can programme metabolism in the long-term in both sexes. Direct exposure to LDD led to smaller adipocyte sizes and lower inflammation in the gonadal adipose compartment of females, and decreased body fat mass in males. In both sexes, digestible energy intake and $\mathrm{H}_{2}$ production were directly increased by LDD. Adult females on a HFD that were exposed to LDD during post-weaning had a better metabolic flexibility and lower macrophage infiltration in the gonadal adipose depot. In males, no programming effects on metabolic flexibility and other metabolic outcomes like body composition and fasting glucose, insulin, and adipokine levels were observed. We concluded that the metabolic programming effects of starches consumed in the post-weaning period are subtle and sexually dimorphic.

I discuss the findings of my thesis and provide implications and suggestions for further research in Chapter 6.

To conclude, we have shown the added value of integrating new gas sensors into a commercial InCa system (Chapters 2 and 3). This technological extension yields realtime, continuous, and automated data that can inform microbiology, nutritional, and other physiological studies. By providing additional information with minimal discomfort 
to the animal, elnCa can contribute to the replacement, reduction, and refinement ( $3 \mathrm{Rs}$ ) of animal experimentation. Furthermore, the data collected in Chapters $\mathbf{4}$ and $\mathbf{5}$ indicates that lowly digestible starches consumed after lactation help to maintain metabolic health during the life course, especially in females. These findings underscore the importance of examining both sexes in nutritional studies, and may contribute to improve the formulation of food products for infants and young children. 



\section{Resumen}



La calorimetría indirecta $(\mathrm{Cl})$ es una herramienta esencial para el estudio del metabolismo energético en humanos y roedores. $\mathrm{La} \mathrm{Cl}$ mide los gases metabólicos consumidos (oxígeno, $\mathrm{O}_{2}$ ) y producidos $\left(\mathrm{CO}_{2}\right)$ por el organismo, lo que se utiliza para calcular el gasto energético (GE). La proporción entre $\mathrm{CO}_{2}$ producido y $\mathrm{O}_{2}$ consumido, o la relación de intercambio respiratorio (RIR), nos indica el tipo de sustrato (carbohidrato, grasa y proteína) utilizado por el animal entero. La precisión y resolución de los sistemas comerciales de $\mathrm{Cl}$ para roedores han mejorado significativamente, aunque poco se ha hecho para ampliar esta técnica para la medición de otros gases fisiológicos. Algunos de estos gases son originados por la microbiota intestinal, como el hidrógeno $\left(\mathrm{H}_{2}\right)$ y el metano $\left(\mathrm{CH}_{4}\right)$ generados durante la fermentación. Por otra parte, la $\mathrm{Cl}$ convencional no permite distinguir entre la oxidación de sustratos exógenos (e.g. provenientes de la dieta) y endógenos (provenientes de reservas corporales). Para hacer esta distinción se pueden emplear marcadores isotópicos estables, por ejemplo nutrientes marcados con carbono $13\left({ }^{13} \mathrm{C}\right)$, y calcular la proporción de ${ }^{13} \mathrm{CO}_{2} /{ }^{12} \mathrm{CO}_{2}$ en el aire exhalado. Es muy poco común que estos gases $\left(\mathrm{H}_{2}, \mathrm{CH}_{4},{ }^{13} \mathrm{CO}_{2},{ }^{12} \mathrm{CO}_{2}\right)$ se midan en estudios con ratones $\mathrm{y}$, en los casos en que se ha hecho, esto ha sido bastante costoso y laborioso.

Los almidones que se consumen en la dieta pueden ser altamente o poco digeribles. Los almidones poco digeribles producen en general una menor respuesta glicémica, proporcionan sustratos para la fermentación intestinal y se cree que previenen la acumulación de tejido adiposo y favorecen la salud metabólica. Hay indicios de que la digestibilidad del almidón afecta de manera distinta al sexo femenino y masculino. La evidencia a este respecto es poca y los estudios realizados en ambos sexos se han enfocado sólo en algunos aspectos del metabolismo de carbohidratos, como pruebas de tolerancia oral a la glucosa y mediciones de glicemia en el estado de ayuno. Así mismo, a pesar de su potencial impacto metabólico, actualmente no existen recomendaciones basadas en evidencia científica sobre la ingesta de almidones para bebés y niños. Esto es importe porque la nutrición en etapas tempranas del desarrollo tiene el poder de condicionar la función metabólica del organismo a largo plazo, un fenómeno conocido como programación metabólica. No se sabe si la digestibilidad del almidón puede programar al organismo metabólicamente, ni si esto puede ocurrir después de la ablactación, cuando el organismo enfrenta un cambio dramático en la composición y densidad de macronutrientes de la dieta.

El presente trabajo tuvo por objetivos 1) desarrollar y demostrar el valor añadido de una herramienta para el fenotipado del ratón basada en la $\mathrm{Cl}$, para el estudio de la actividad de la microbiota intestinal y la oxidación de sustratos exógenos us endógenos en tiempo real, y 2) aplicar esta herramienta al estudio de los efectos directos y a largo plazo (programación metabólica) de los almidones consumidos durante el periodo inmediato tras la ablactación.

En el Capítulo 2, nos propusimos estudiar la actividad de la microbiota intestinal de manera no invasiva, continua y en tiempo real, mediante un sistema de $\mathrm{Cl}$ aumentado (CIA) con 
sensores de $\mathrm{H}_{2} \mathrm{y} \mathrm{CH}_{4}$. En ratones alimentados con una dieta basada en almidón poco digerible (APD) o almidón altamente digerible (AAD), la producción de $\mathrm{H}_{2}$ mostró un ritmo circadiano dependiente del consumo de alimento y de la digestibilidad del almidón. La cantidad de $\mathrm{H}_{2}$ producido fue suficiente para explicar el $20 \%$ de la variación taxonómica en bacterias fecales y se correlacionó con géneros específicos de bacterias que se sabe son productoras de $\mathrm{H}_{2}$ in vitro. No se detectó producción de $\mathrm{CH}_{4}$, lo que concuerda con la ausencia de arqueas metanógenas en heces. Concluimos que la $\mathrm{CIA}$ es una herramienta útil para el estudio en tiempo real de las interacciones entre la dieta, la microbiota intestinal y el huésped.

Posteriormente incorporamos sensores de ${ }^{13} \mathrm{CO}_{2}$ y ${ }^{12} \mathrm{CO}_{2}$ en el mismo sistema de $\mathrm{CIA}$. El Capítulo 3 tuvo por objetivo demostrar el valor añadido de estos sensores. Con este sistema fue posible detectar diferencias en el enriquecimiento en ${ }^{13} \mathrm{CO}_{2}$ correspondientes al enriquecimiento natural en ${ }^{13} \mathrm{C}$ de la dieta y los patrones de alimentación. Usando los datos combinados de enriquecimiento isotópico y $\mathrm{Cl}$ convencional, pudimos cuantificar la tasa de oxidación de una dosis ${ }^{13} \mathrm{C}$ glucosa $0{ }^{13} \mathrm{C}$ palmitato administrada a ratones con diferente masa grasa corporal, independientemente de las tasas de oxidación de glucosa y grasa en el animal completo (la suma de sustratos exógenos y endógenos). La oxidación del palmitato ingerido se correlacionó negativamente con la masa grasa y positivamente con la flexibilidad metabólica del animal. Concluimos que habilitar el análisis de enriquecimiento en ${ }^{13} \mathrm{CO}_{2}$ como parte de la $\mathrm{ClA}$ la convierte en una poderosa herramienta para estudiar la oxidación de sustratos específicos cuantitativamente en condiciones fisiológicas y patofisiológicas.

En el Capítulo 4 nos propusimos probar la hipótesis de que la exposición a una dieta basada en APD o AAD influye en la capacidad para oxidar la molécula de almidón, e investigar si estos efectos son similares entre hembras y machos. Los animales que consumieron APD durante 3 semanas oxidaron una mayor proporción de una dosis de almidón gelatinizado (altamente digerible) que aquellos que consumieron AAD previamente. Este efecto fue mayor en las hembras que en los machos, puesto que la cinética de oxidación del almidón fue más acelerada durante la etapa postprandial temprana en las hembras alimentadas con APD us AAD, lo que no se observó en los machos. Sin embargo, los machos alimentados con APD sostuvieron una RIR más alta, tanto en condiciones de ayuno como durante el periodo postprandial después de consumir la dosis de almidón. La oxidación más completa de esta dosis de almidón en hembras alimentadas con APD no pareció obedecer a diferentes niveles de amilasa intestinal. Con base en estos datos hemos concluido que el consumo de almidón poco digerible aumenta la capacidad para oxidar la molécula de almidón a corto plazo, especialmente en las hembras.

Nuestro objetivo en el Capítulo 5 fue demostrar nuevamente algunos de los efectos directos de la digestibilidad del almidón así como investigar si una dieta basada en APD - AAD inmediatamente después de la ablactación puede programar el metabolismo de machos y hembras a largo plazo. En el corto plazo, las hembras que consumieron APD desarrollaron adipocitos de menor tamaño y menor inflamación en el depósito adiposo gonadal, mientras que los machos alimentados con APD mostraron una menor masa 
grasa corporal que aquellos que consumieron AAD. Así mismo, el consumo directo de APC llevó a una mayor ingesta de energía digerible y una mayor producción de $\mathrm{H}_{2}$ en ambos sexos. En la etapa adulta y durante el consumo de una dieta alta en grasa, las hembras que estuvieron expuestas a la dieta APD después de la ablactación mostraron mejor flexibilidad metabólica y menor infiltración de macrófagos en el tejido adiposo gonadal. La flexibilidad metabólica de los machos no fue programada, ni tampoco otros parámetros ligados a la salud metabólica como la composición corporal o las concentraciones en sangre de glucosa, insulina y adipoquinas en el estado de ayuno. Concluimos que la capacidad de los almidones consumidos inmediatamente después de la ablactación para programar el metabolismo es sutil y sexualmente dimórfica.

En el Capítulo 6 discuto los hallazgos de esta tesis y sus implicaciones y también ofrezco algunas sugerencias para futuras investigaciones en el campo de la programación metabólica.

En conclusión, hemos demostrado que integrar nuevos sensores de gases en un sistema comercial de $\mathrm{Cl}$ añade valor a esta técnica (Capítulos 2 y 3). Esta extensión de la tecnología proporciona información en tiempo real, continua y automáticamente, que puede ser valiosa en estudios microbiológicos, nutricionales y en otras áreas de la fisiología. Al proporcionar información adicional con mínimas molestias para el animal, la CIA pude contribuir al reemplazo, reducción y refinamiento (principio de las $3 \mathrm{Rs}$ ) en la investigación con animales. Así mismo, los datos reunidos en los Capítulos $\mathbf{4}$ y $\mathbf{5}$ sugieren que los almidones poco digeribles consumidos después de la lactancia ayudan a preservar la salud metabólica a lo largo de la vida, en especial para las hembras. Estas observaciones subrayan la importancia de incluir ambos sexos en estudios nutricionales, y podrían contribuir a mejorar la formulación de los productos alimenticios destinados a bebés y niños. 

Acknowledgements  

$K_{t}$ long last! At some po int I thought I wouldn't make it. But here it is, the finished book, a few papers, and $100 \mathrm{~s}$ of lessons learnt. Some of those lessons were rather painful, ard some were unexpected. But AKL of then I appreeiate now, along with eveny omeaning ful thing that people have said on done to make me happy duning my PhD... Here's a list, feel free to skip right to your name:

- Kim: Gracias x esperarme hasta el momento en que empezaría mi verdadtro despegue como achulto. Gracias x ser tú en loque pienso cuando ttengo" que poner una sonrisa en mi cana (tú sabes a qué me refieno). Tededic esta tesis.

- Ja2p: I think I'm only beginning to sae what you tried to do with me, and I'm grate. fal in every way. All your comments, from the many times you criticiged the way I delivered my presentations, to those other times when you intereupted omy sentences, to the awkwand moments when zow spotted my lousy (sic.) orguments... All of these are now gold to me. These difficult interactions showed me how to use my presentation skills effectively, and what then felt like underappreciation now became pant of my strenyths as 2 scientist. And let's not think about where I'd be now had you a lways let me canng on talking, because I realise how much I really needed to listen back then I actually ave a great deal of my scientific output to Knowing to keep quiet and trust you (that $\mathrm{H}_{2}$ papen and some data in the ${ }^{13} \mathrm{CO}_{2}$ paper were 21 most serendipitars, and I'd have missed the oppontunity if I hadrit had your experience on myside). From you I learnt how to read a papen in 5 minutes and how to think in terms of "Reseanch guestions." One thing I didn't guite learn, I see, is to Keepit simple. So this list could go on ad infinitun but I'd better stop night hene.

- Evert: whene to start? The first thing that comes to mind is: thank you for taking me sooo o seriously. There was not a single moment when what I did on say was indifterent to you. Yuu supenvised ne closelyand carefully, and I know not many PhO candidates can get that much a ttention from their supervisons. Of course, we had 2 few disagreements and I got mad at you, but all the time you were "only" doing your job . No matter how stubborn I was, you were always patient and polite, and you were more than ready to provide feedback. Thanks to your feedback my uniting is much better and scientific, and my Ppts are always on po int. Mone than once I got more feedback than I asked fon, and I consider myse if lucky that you tock the time to pre pare it fon me as pant of my scientific training. A good example is the way you helped me see the value of my work and be veny confident in it. Imespecisally gratefwl because under your academic wing I felt sate to make mistakes and grow as a soientist, because finst and foremost you saw me as a human being. And thank you for lending me 2 hanfful of ne as earch guestions from which to stant off my academic journey Evert, if there was only 1 thing I could thank you for, that'd be the way you mever gave me ordens, but instead encouraged me to think cuit:cally. Atimes it felt like every step was a huge deal: wothing could be clone on even writeh withou-l prior conswltation. But it is becouse cou took me so seriarsly that now I'm as rigonars and skeptical as a true scientist should be. Maybe you didrit even plan to be such a tercher to me, but I find it beauti- 
tul that I Keeplearning from you to this day (I think the la'st thing was about multiple testing...). Thank you for shaping my iden t: ty as an 2cadernic, and Krow that I'll 2 lways see 2 rele model in yolw. Funny thing is, this is the only part of this thes is that you canot edit, but something tells me you'd approve :)

- J22p and Event, to both of you: I treasine every noment when. Icould cleanly see yow passion for the tnuth, when I could see that I meant much more to you than 2 way to boost you H-indices. I lived for the times when I saw you makvel at nature and the understanding of the Univense. Thank you for this, and for staying late at many of uur wonk discussions. This was to me, in 2 way, 2 "critical period" of my scichtific development. "Cu've shaped me you've "programmed"me, and I'm grateful fo n hawn influence on my "academis pheno type" "you were my mentors, and I hope you continue to be a source of $2 \mathrm{mus}$ som and suppont for the ycarer

- Annemanie: A lat of what I said to Event and Jaap applies to you. Youive also been 2 majoun enviponmental cue" in my development, 2 briliant one at that. Thank you for being the strong, yet sensitive roice of neason. Youn feedback neven failed to refresh me, always direct but positive and construct ive. I really enjoyed when you came to on meetings, those days were exciting and filled me with energy. Why? I dunno, I suppose that's your supen power. But you really yave me comfont, and I'm sure you know what I mean. Thank you!! :)

- Lianne: I really value having wonked in the same project togel har. We had our differences, but I leasnt 2 ton from them. I apprecia te how you were sensitive to my'fears, like the many times I felt stupid in the 126 . Or how you were really prodative in the deas ions we had to makle, even when they concerned my sown' studies. Cou may not knaw it, but I'm veny grateful for your attitude dining the dissections. You canmanded', us arcund with sech con fidence. Ireally needed you to be strong, and you picked up on it immediate. $l_{y} . .$. I espevishy remember ore time whon Meligst was late and yu daved the day. Thank you! And also thank yau for reading my clnafts so very carefully. yar spotted things that not even the mone senion among us did. Andyow heiped me overcome some ct mes abuse of the article "the.". Lianne, thank you for being yourself. I admine you and I miss you. That's why I some'times say to myself. " $\omega \omega L D$ ?" when I need to feel like a boss in the 126 . Thank you so much for baing my inspina tion.

- Wenbia 0: It was always good to talk to yeu. Iappreciate on $\sim$ discussions about science, acadomlia, and wir tife gals. Tou always geemed to be in 2 good mood youn ever-smiling face gave me 2 warm feeling that it is poss ible to do your PhD without losing your mind. Thank you for making time for helping me with my mice even when yal realy didr't have it, andfon thinking along with me abaut my data. I now see yau 2s someone with guite some expenience, and I thank you fur being yo neady to share it with us who still have 2 lat to learn. I ancharored to have been your paranymph, because I could setuahy do something to make yaur celebration happen. A celebration of the effurts we young" scient ists do to take a place in this whinsical system. I respect you Wenbizo, and I hope we can Keep building one friendship within or autside academiz 
- Anjan, Rund, Harny, Henny, Nils, Bent and Jöng: thank You for contributing yaur insights during owe TtW meetings, and 2lso outside these meetings. I thirk you repres ented much more than the institutions/companies you wonk (ed) for, because you neally showed a genuive interest in our project and the develop ment of me and 2 ianne as scientists. Thank you formathing on project pussible and for makiry eveny meeting 2 rather enjoyable one. I hope our efforts continue to pay off and benefit science \& society.

- Ries: You're part of the paragnaph above, but I think you really went the ex tramile. I thim we matched very nicely at some point and I really enjoyed talking to yau and shaning our first results. Yon know how excited I was about the whole iso tope thing, and it was nice to have someone share that feeling. Thank you fur trying to make my PhD as smooth as possible by pointing me in the night dinection and even gluing me some speople advices. I really hope I can do some thiry mith Isolife in the tutwe!

- Vincent: we didint talk much, but when we did it was Re treshing. When you came to the group I think you brought a new dynomic. Ifelt you were a trully impartial, experionced scientist who cuuld criti. cize my work. I lock forword in particulan to hearing oreciscly I also thank you for introducing a bit of politeness to the group, 2 t leadt the Kind I'm more ujed to. As mimings I fove a direit drathenge, sometimes one really needs to hean a positive comment when receiving feedback. Thank you for being that person, Vincent.

- Jeske: Your selt-confidence and carefree apporo ach to life was a Reminde a to me that thing's den't have to be ternible all the time. It's mainly how we see then. Ill always nemember that nia flowchant you had on your wall abat wo najing. Thank you for bringing me coffee sometimes and for encougging me to joir the coffee brealks - Lonncke: You were also velry helpful. I really liked having around someone so chatty and sociable. I really liked the time you invi ted me to you nflace while I was doing the laundry. And I'm gratecfull for the adive you gave me when I asheed you about getting ar extension for my PhD. - Matasja: Youre arother person I admine. Thank you for shaling statistical tips \& tricks with me, and for all the times you helped with our disscutions. 


\section{ACRNOWLEDGEMENTS}

At times you were not so-sociable, but whenever you were you always made me layg. Irealize we have a few things in common, something in our sense of humour perhaps. Ow our love of pigs and cats, and our dislike of spiders. Any how, I believe you'll be a great scicnt ist and I hope yau have mamy stu dent s whom ya can teach how to do good science. (Asso, you're really smon $t$ and I'm jealo us).

- Taolin: Thank you for being kind and helpful. I see yau work very hard and I admine you. And I mus t say having you in oun gnoup helped me relay a little and come to the cottee bie aks more of then I liked how sweet \& wite you'reat times, because that made me feel good U' - Aunna: Another "refreshing" penson. Yau're cool, smant, and socialele. While I'm us riting these av-nowled gements I realise how much I aeally appreciate having youguys around. Sumehow you and the othen PhD ens have gua lities I don't, and that really made the group a very nice plaee to wonk. I think you represent this feeling of mire, the gratitude to have a diverse group of peers to which I can campare my self with and think in ways I can improve as a penson. Thank you Anna, and thank you all of the other PhOers: Li, Marjanne, Bant \& Joellle. - Sheyin: During your time with us I leannt 2 lot about myself just by taking to you. Yau'll probally wor't see this, but somehar I still need to thank you for giving me a few life lessons.

- Nara: itemiga Nara guerida! the un deleite tererte en HAP contimuse muestra amistad frens delgrupo. Reverdo cor cariñolas veces gue tomábames un cafeciuto en Judiac y me platicabas de th his toria de vida y las metibeiores. Eso me marco'. Te guiers mucho y te agradezce por tratarme con caiño, me hacía muche falt2 en el momento en que leyaste. Mesé 2 dinde nos leuans' la cicicia (o la vida), pero ojzlá que muers tros camines se crucen felizmente. - Omarúa. Hap two el gusto de conocerte po r relativamente poco tiem oo, pero yo me quedo con may gastos rewerdos de ti. Me gus tó mucho cono nos renarimos en the casa para Eunovision, y las veces que platicamos de la ualay el trabajo. Gracias por darme un espacio para hoblar er mi idioma e intereambiar impresiones. Te admiro mucho y espero gre siyarres en conzacto mucho tiempo ma's. - Silvie, Oorier, Jessica, Marcel, Kakja, Anie, Sander, Annet, Annik2 and Melissa: Thank you for baing nice colleagues and helping me!! 
- Iyzbo: You, nny friend, are special. I still can't think of a better example cf 25 trong woman. Proving over here with a fanily to care for and $2626 y$ in your womb is really something else, and you had to faee an entinely difterent cel tire ard way of wonking ah at the same time. Trying to do 2 PhO is hard erougl. I am glad juu reslised you're veny smart and capable, and tust you've decided to stand upfor yourself. Not wery PhO candiclate has the same privileges, and I will aluays respect you for hoving the currage to take on this dhallerge. Pleose don't even let those barriers guestion your wouth as a person. Thank you for being a very preciass friend

- Hans: A lot has been said already when you retired, but IIll say it again. Yau are ove-of-2-Kind. Mn any times I lost it when we wonked together, but looking back. I see how much I learned from it. I still miss you where I am now. Ineed someane who can share with ne every thing that so many years of expewience has taught him. I really liked how you oknays hied to make our science better. If I didist seeit back then now I do, and I thark yau for it! What's more, you sre a Kind and roble person, and I hape I have more colleagues like you in the future.

- Inge: I think you aluays had an opt.mistic mind. Or at least all of our interactions were like that, and I really apprecate it. Derhaps becanse of that att: tude, more than one I came to you with my "P $\gg>0.05^{\circ}$ Kind of da'tla and you helped re see value in them all the same. I hope you continue to guide many scientists in training like me because you have a lot to texch them! thank youll - Annelies: I could urite auhde chapter about you. We dar't have that Kind of budget, so IIII just say Iim grateful for all the times you came to "unghe" me f'rom my aumpuler to joun the coffee breaks. And one mone thirg: that tive I had a personal situation at home, you were the only one who cared to listen to me. I'll always rememben this, and I thank you for showing me that you caned.

- Conine, Irene, Anrel \& Annika: I think I wouldrit have been able to deal with all the preerwonk and logistics involved in my PhO without 
Your professional help. You all made my life easien and I would not like to see it as a giver, but as 2 gift fromyou to me. Thank you for being excellent and helping onngrap stay unganised. - Eua, Auke, Lini, Launa, Curike, and Janya: I learnl a lat from you. Being a supenvisorcan be really scany, but you made it fun. You all were really good at pointing out my deegest feass and weaknesses, altho you didn't no tice. So, besides thanking you for the hard wonk you put into our prgact (especally at (KP), I thank you for being my teachers. - Yurike: I thank you for the hand wank you did. Withowt it, I wouldn't have been able to finish every thing in time. I wish I could show my gratitude in arother way, but please know yan've trubly cowtributed to the programming field

- Pever de Gijsel \&michel Brever. Yuu must be the most patient people in alolland. Thank you fortahing me through all the intricate technicali ties I needed to unders tand at some point.

- Prokopis: It was great to wonk with you and finally unders tard some reatly high-level concepts about data scieree thanks toyan explanation. your hard wonk added a veny interes ting per spective to un a paper. I hope my future collaborations withother scientists could be like this one. - Hauke: A lot of what Isaid to Prokopis applies to you as well, but I must say your willingness to take my ideas scrously was remankable. Thank you for being an approachable professon.

- Wihna, Bert \& René: The CKP was my second home, and you always made sure it was tidy. Evenmere so, oun precious experimental units lived in a hux resont thanks to youn care. On behalf of all of the mice and 21 so of the ones I will keep formy future studies, thark you for helping me prionitise animal welfare above every thing else.

- Arturo: Gracias por ayudarme a ver la realidad, curo siempe.

- Rosame: i Ami ga guenidal Gracias por sermi mejor amiga ho landesa y por comprender lo ocupato gue esture durante el doctorado. Sabes que te guiero mucho y gue estoy my aradecido por que me aecompañaste en todo momento. Pasaron muchas cosas en 4 aŕos, unas my difíciles, pero siempre estur imos el uno parael otro. IGracias, te quieno muchol! 
- Elir y Adriancito: Ustedes tueron parte del proceso. Gracias por siempre estar super listos para pas anrosla nico y dis frutar de las atras cosas de 12 vida, no sólo el trabajo.

- Maru y Joost: Gracias pon apayarme TANTO. Todas esas chanlas sobre las justicias e injus ticias del mundo fue ron un gran divio para mi. Ustedes saben le difícil gue fre esto 2 ratos, y me tuvic non 12 preiencia para esperarme a gue twiers tiempo para salin. Suapayo no sólo esturo commigo dirante el doctorade, sino desde antes de empezar la ma estría. Graias pur hacenmé un espario en ar casa yen bus $21 \mathrm{mas}$. I Joost, tur me ins pinas a sen un científic ma's chingón. Cuando me dijiste gue "I ciencia 2vanza Sol2", supe gre tenemos mucho ma's en comín de 6 que pensó. Sigamos dejando gue 12 gente sc pelee gor los galardones mientras desarb iomos la naturedza y maw, th' has side mi mejor mentora en este pás sobre cómo detender mis derechos. Tiu y jost, sin duds, sun personas gue me har ayudado a crecer $y$ ser un buen Ber humano. iLos guieronucho!

- Cristóbal/Ximena: Caué decin! Sabes que esto me costó mucho. me viste hacer entri pados con cada jalada gre sucedió, y apesar de mi agenda tan caphichosa ahr cestujiste, sicmpare invitándome a 8 way o todos esos hyares tan... peculiares. Desde la maestría has sida tú la rafón poula que perdí la inocencia, pere gané muchas experiencias gue ro mubiera canccide sinti. Grocias por todo tu coniño y temmuna y por tadas las risas y momertos "máximes" que heno s vivido. ite Guiero mucho!s - Jorch: La mamá de tod@s agú. Si compre fuistemey cindo, pero durante mi doc torado sacaste tu verdadero ya Una calidad humara como pucas, una manera de hacerme sentir tan pero tan quervido... Mo sć! No me explico cómo puede haben algiven como tú. Enes una person 2 my bella forch. Siento gue me guiveres mucho yme entiendes my bien. Gracoss pou todes tus consejos sobre cómo ser un adul to en Hobuda. Gracias por be amistad y por quererure tanto. iiito tambión te quiero!!!

- Paraminfas: Graess x ser tan chinganas. Kthr6ai. (si pudiera poner cu "mishi triske" agu', saben gre lo haría.)

- Leo y Juve: C mamaron. Nadie logró legar hasta acá, pero ustedes

sí que hicieron el es fuerzo. Son unos reyes, y no por nada mis 
184

ACKNOWLEDGEMENTS

amejones (y más vigos) amigos Gracias paor todo lo gue hemos vivido juntos, y oor apyarme en mi decis ión de viajar hasta aca' para continuar con mis es tudios. Quiero que sigameos haciéndolo todo para caltivan nuestra amistad y segain siendo una bonita infwenaia sobre muestras vidos, angue sea des de México, Holanda o Estados unidos. Los givero mucho y no saben lo que signi ficapara mú que hayar venido a celebrar conmigo. Este es el mejor regalo que me han hecho y rui corzzón se leasz de dicha de tenerlos agú.

- BRO: Cómo duvidarlos! Gracias muchas veces a ustedes he podido reínme en nomentus feos. Descle que me fuide mestro paús han guerido seguir en contalto $y$, angreno siempre $b$ puede, seguimos con la tradición de reuninnos al menos una vezalaño. Graciss Anis, Eves, Annulfis, Garci, y ttfrede por ser tan avistosos y hacer 'de 12 vida 2 lgo más amena Los extraño mucho y guissers que se reunieranmais y me invitaran, anngue fuera vintua (mente. Y mis Kokonitos Gunaudiare y Modia, son ustedes una fuente de alegrús y ternura Los Hevo siempra en mi corzzón. Ya no se alejen tanto; podemos estar juntos desde la distancizi i los a modoro!

- Don manuel, sna. Rosalla, Srita Deni, Marecita y Chris: Gracias por reciburme cadague vay a Créxico. San como mi $2^{2}$ familia y doy gracias a Loges porque Omarolito es corro es por habor crecido en un lugar don de hay tanto amoriil - Dra. Rosanio i Maestra! Esporti que amo la ciencia y la proy ramación metabólica. Oe ti fue guien es cuché el témunino por primera ver. Todo, resmente todo, lo que aprendé contigo me hasyudado en este doc torado y a 10 largo de mi formación. Gracias pouser mi mentora y por transmitime todo ese compromiso que ticres par ser una buena científica. Tequierd mucho.

- Lorge Maldenzolo: Otro de mis g randes mentares. Ojala que te pueda entregar mi tesis en persona pranto. La parte de los iso' topos te 12 debo en pante ati: no me hubiers ido tanbien si no me hubieras enseñado lo que son. Muchos trueus los aprendé de ti, $y$ te lo agradegco in finitamente. iGracos por sen mi maesto? 
- Familia: Oni apoyo desde siempre. Gracias a Des: y José pur venir hasta acá. Es como simi greaida Andy estwienz aquí. Jue graeizs a ella gue mestra familia se unió ma's Es toy miy agradecido con custedes por que gracias a su compañit mis papás no se quedaron salitos. Gracias por estar con nosotros y ser nuestra famila. Los guvero mucho y ogalá sigamos uniéndonos muchomás aín. YZun beso hasta el cielo para Andy. \$ Y (muchom is gue) "un saludo a mi abuelita". - Dadito del aman Mow. Gravias par hacer este trabajo tan borito. Nos hizo sufrir, pero quedó may bacito. Gracias por ayudarme a transmitir mi mavera de persar en forma de este diseño tan pode. noso. Admind lu tatento. $y$ sobre todo admiro tumanerz de ser quien erves. Grocias por sianpre dame amory sabe que cste es el más bello regalo que me pudiste dor. Gracias por ser mi hermanita y por dorme tanto tanto amon. iTe quivero cmuchol - Pez y Monsi: Aus. No alcanzarían las páginas para agradecerles su amon y apayo. Lo gue soy se los debo 2 us tedes. Sin el hogar tan nico gre hicicron para Yuyo y para mí no creo que hubiers tenido Las ganas de lograr un sueños tan noble como cste gue es 12 ciencia. Doy gracias por cómo me lo dieron todo para ser un hombre que le prede apontar algo bweno al mundo. Los guicro mucho y creo que sólo we gueda sey uin cuidóndelos y netribuyóndeles taclo le que, mehan regalado y, Jobre todo, seguin dis furtándoles ojalz' qué por muchos zinos mós. 2 os amo.

- P.S./Manolito: El áltimo agradecimiento es parati. Como en los antículos científicos, muchas veces el ciltimo cozator no ha hecho el trabajo suciv pera s. hoha inveratido todo en él. Thi a postaste todo por amí, me tuviste fe yo me diste th consejo en todo momento. Estuwiste cada una de esas mos de dos mil maĩaras para darme tu amar des de OMéxico desde el primer día gue me fwi a vivir fuara. Gracios a ti nunca estuve realmente solo gan lejos gue estwic re. Gracias por avidar 2 mis papitos ya-yuyo, y por compontinme de tu hamosa pamiliz. I sobre todo, gracias por ser un espejo del alma que me ayuda a prae. ticar la vintud y lleis unz vida bien vivida. Cjola gue pudames

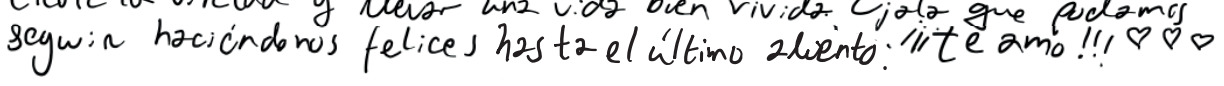



About the author  



\section{Curriculum Vitae}

José María Salvador Fernández Calleja was born on the $15^{\text {th }}$ of June 1988 in Mexico City, Mexico. In 2006, he completed his pre-university education at Universidad La Salle in Mexico City. In the same year and at the same institution, he started a BSc programme in Food Chemistry. It was during his BSc thesis work at the Mexican Institute of Social Security, analysing the lipid quality of infant formulas under the supervision of his "academic parents" Dr. Rosario Ayala Moreno and the researcher Dr. Jorge Maldonado Hernández, that he first encountered the promising field of early life nutrition and metabolic programming. After obtaining his BSc degree and a short flirt with the pharmaceutical industry in 2012, José decided to continue his scientific education abroad. Thanks to a scholarship from the Consejo Nacional de Ciencia y Tecnología (CONACYT), he moved to the Netherlands to start the MSc programme Molecular Life Sciences at WUR on the same year. Home sickness ensued, but this was the intention. During his MSc studies, José was lucky to have his first serious attempt at molecular biology supervised by Prof. Sander Kersten of the Nutrition, Metabolism and Genomics group, where he investigated the role of a musclederived humoral factor in insulin resistance. Because he was already too comfortable among the Dutch, José then sought an MSc internship in Aberdeen, UK, where Dr. Perry Barrett trusted him with his all-important Siberian hamster brain samples to analyse the seasonal gene expression patterns using dangerous radioactive probes. This was excellent preparation for what was to come, as soon upon his arrival back in the Netherlands in 2014 he landed a PhD position at WUR's Human and Animal Physiology group. Molecular biology, rodent studies, and isotopes were all exciting parts of the game but, more than anything, José was enamoured of the metabolic programming focus of the proposal. His patient PhD supervisors and sparring partners, Prof. Jaap Keijer, Dr. Evert van Schothorst and Dr. Annemarie Oosting, taught him a lesson or two (actually, too many to name, all of them welcome) on physiology and how to conduct oneself as an ethical, truth-seeking scientist amidst the contemporary "publish or perish" academic culture. During his PhD José himself had six pupils who gave him a rewarding taste of the pleasures of teaching. After this critical period in his academic training, a sort of second adolescence, José came out as a young investigator and sooner than later other scientists too acknowledged his passion and skills to comprehend the Universe. José is currently enjoying his first postdoctoral position with Dr. Lucianne Groenink at Utrecht University's Pharmacology group, where he hopes to keep contributing to improve the developmental trajectories of young children through metabolic programming. 


\section{List of Publications}

R. Ayala-Moreno, J.M.S. Fernández-Calleja, J. Maldonado-Hernández. Assessment of lipid quality and composition of commercial infant milk formulas in Mexico: Emphasis on trans fatty acid isomers. Food and Nutrition Sciences 2016; 07:273-283. DOI:10.4236/ fns.2016.74029

I. Petri, V. Diedrich, D. Wilson, J.M.S. Fernández-Calleja, A. Herwig, S. Steinlechner, P. Barrett. Orchestration of gene expression across the seasons: hypothalamic gene expression in natural photoperiod throughout the year in the Siberian hamster. Scientific Reports 2016; 6:29689. DOI:10.1038/srep29689

J.M.S. Fernández-Calleja, L.M.S. Bouwman, H.J.M. Swarts, A. Oosting, J. Keijer \& E.M. van Schothorst. Monosaccharides in post-weaning diet of young mice program body composition and feeding behaviour in adulthood. Scripta Scientifica Pharmaceutica 2017; DOI:10.14748/ssp.v4i1.3962

L.M.S. Bouwman, J.M.S. Fernández-Calleja, H.J.M. Swarts, I. van der Stelt, A. Oosting, J. Keijer, E.M. van Schothorst. No adverse programming by post-weaning dietary fructose of body weight, adiposity, glucose tolerance or metabolic flexibility. Molecular Nutrition \& Food Research 2018; 62(2):1700315. DOI:10.1002/mnfr.201700315

J.M.S. Fernández-Calleja, P. Konstanti, H.J.M. Swarts, L.M.S. Bouwman, V. GarciaCampayo, N. Billecke, A. Oosting, H. Smidt, J. Keijer \& E.M. van Schothorst. Non-invasive continuous real-time in vivo analysis of microbial hydrogen production shows adaptation to fermentable carbohydrates in mice. Scientific Reports 2018; 8:15351. DOI:10.1038/ s41598-018-33619-0

J.M.S. Fernández-Calleja, L.M.S. Bouwman, H.J. Swarts, A. Oosting, J. Keijer, E.M. van Schothorst. Direct and long-term metabolic consequences of lowly us. highly-digestible starch in the early post-weaning diet of mice. Nutrients 2018; 10. DOI:10.3390/ nu10111788

L.M.S. Bouwman, J.M.S. Fernández-Calleja, I. van der Stelt, A. Oosting, J. Keijer, E.M. van Schothorst. Replacing part of glucose with galactose in the post-weaning diet protects female but not male mice from high-fat diet-induced adiposity in later life. The Journal of Nutrition 2019; 148 DOI:10.1093/jn/nxzO28.

Accompanied by a commentary: J.S. Ruff. Galactose-mediated protection from metabolic disease: nutritional programming, parental effects, and the milky way forward. The Journal of Nutrition 2019; doi: https://doi.org/10.1093/jn/nxz080.

J.M.S. Fernández-Calleja, L.M.S. Bouwman, H.J.M. Swarts, A. Oosting, J. Keijer, E.M. van Schothorst. Extended indirect calorimetry with isotopic $\mathrm{CO}_{2}$ sensors for prolonged and continuous quantification of exogenous us. total substrate oxidation in mice. Scientific Reports 2019; 9:11507. DOI: 10.1038/s41598-019-47977-w.

J.M.S. Fernández-Calleja, L.M.S. Bouwman, H.J.M. Swarts, N. Billecke, A. Oosting, J. Keijer \& E.M. van Schothorst. A lowly digestible-starch diet after weaning enhances exogenous glucose oxidation rate in female, but not in male, mice. Submitted. 


\section{Overview of Completed Training Activities}

\section{Discipline-specific activities}

Course, Laboratory Animal Sciences

Utrecht, NL, 2015 (UU)

Course, Metabolic Phenotyping of Mice

Düsseldorf, DE, 2015 (EASD)

Conference \& (3) oral presentations, Dutch Nutritional

Heeze, NL, 2016-2018 (NAV)

Science Days

Symposium, Nutritech

Lisbon, PT, 2016 (NutriTech)

Course, Chemometrics

Wageningen, NL, 2016 (VLAG)

Course, Indirect Calorimetry

Cracow, PL, 2016 (WUR-INRA)

Conference \& poster presentation, The Power of

Munich, DE, 2016 (ENA)

Programming

Course $\&$ poster presentation, Intestinal Microbiome

Wageningen, NL, 2017 (VLAG)

Course $\&$ poster presentation, Epigenesis and

Epigenetics

Conference, workshop, \& poster presentation, $\mathrm{DOHaD}$ World Congress

Conference, course, $\&$ oral presentation, NuGO Week

Wageningen, NL, 2017 (VLAG)

Rotterdam, NL, 2017 (DOHaD society)

Varna, BG, 2017 (NuGO)

\section{General courses}

VLAG PhD Week

Baarlo, NL, 2015 (VLAG)

PhD Workshop Carousel

Wageningen, NL, 2015 (WGS)

Course, Teaching and Supervising Thesis Students

Wageningen, NL, 2016 (ESD)

Course, Scientific Publishing

Wageningen, NL, 2017 (WGS)

Course, Interpersonal Communication

Wageningen, NL, 2017 (WGS)

PhD Peer Consultation

Wageningen, NL, 2017-2018 (WGS)

Course, Philosophy and Ethics of Food Science and Technology

Wageningen, NL, 2018 (VLAG)

\section{Optional activities}

Preparation of Research Proposal

Wageningen, NL, 2015

HAP Weekly Group Meetings

Wageningen, NL, 2015-2018

HAP Journal Club

Wageningen, NL, 2016-2017 
The research described in this thesis was financially supported by the Dutch Technology Foundation STW (13509), which is part of the Netherlands Organization for Scientific Research (NWO), and which is partly funded by the Ministry of Economic Affairs; and by the Consejo Nacional de Ciencia y Tecnología (CONACYT, 311576), Mexico.

Financial support from Human and Animal Physiology for printing this thesis is gratefully acknowledged.

Cover and graphic design by Giuliana Fernández-Calleja

Printed by Digiforce | ProefschritMaken 

